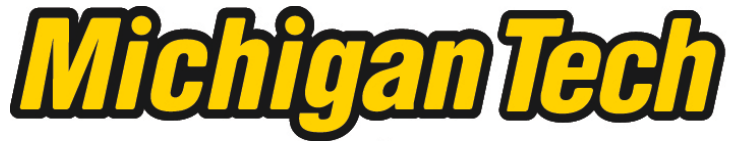 \\ Michigan Technological University Create the Future Digital Commons @ Michigan Tech
}

Dissertations, Master's Theses and Master's Reports - Open

Dissertations, Master's Theses and Master's

Reports

2014

\section{DESIGN, SYNTHESIS AND APPLICATIONS OF FLUORESCENT AND ELECTROCHEMICAL PROBES}

Giri K. Vegesna

Michigan Technological University

Follow this and additional works at: https://digitalcommons.mtu.edu/etds

Part of the Biology Commons, Chemistry Commons, and the Materials Science and Engineering Commons

Copyright 2014 Giri K. Vegesna

\section{Recommended Citation}

Vegesna, Giri K., "DESIGN, SYNTHESIS AND APPLICATIONS OF FLUORESCENT AND ELECTROCHEMICAL PROBES", Dissertation, Michigan Technological University, 2014.

https://doi.org/10.37099/mtu.dc.etds/931

Follow this and additional works at: https://digitalcommons.mtu.edu/etds

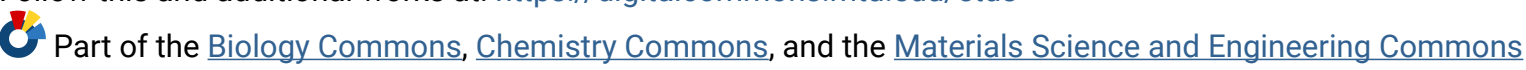


DESIGN, SYNTHESIS AND APPLICATIONS OF FLUORESCENT AND ELECTROCHEMICAL PROBES

\author{
By \\ Giri K. Vegesna \\ A DISSERTATION \\ Submitted in partial fulfillment of the requirements for the degree of \\ DOCTOR OF PHILOSOPHY \\ In Chemistry
}

MICHIGAN TECHNOLOGICAL UNIVERSITY

2014

(C) 2014 Giri K. Vegesna 

This dissertation has been approved in partial fulfillment of the requirements for the Degree of DOCTOR OF PHILOSOPHY in Chemistry.

\section{Department of Chemistry}

Dissertation Advisor: Dr.Haiying Liu

Committee Member: Dr. Ashutosh Tiwari

Committee Member: Dr. Shiyue Fang

Committee Member: Dr. Xiaoqing Tang

Department Chair: Dr.Cary F. Chabalowski 



\section{Table of Contents}

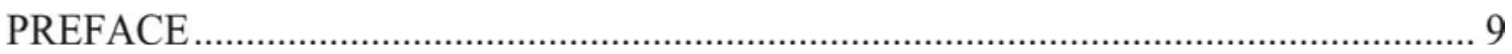

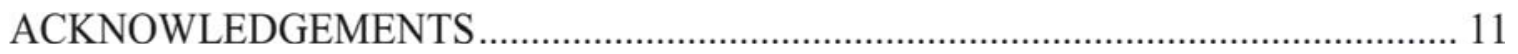

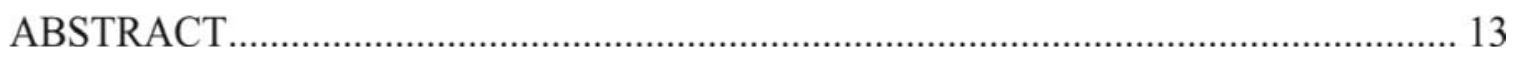

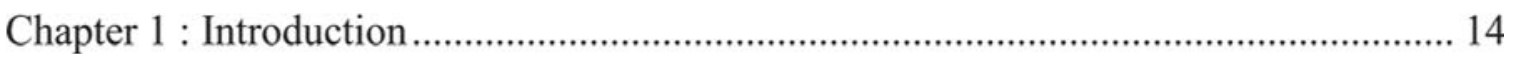

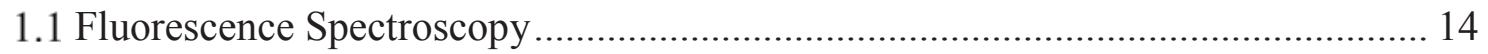

1.2 Small molecule fluorophores for labelling biomolecules ............................... 16

1.3 Small molecule fluorescent probes .......................................................... 17

1.4 Design principles based on different fluorescent modulation mechanisms............ 18

1.4.1 Photo induced electron transfer (PET) based probes ..................................... 18

1.4.2 Intramolecular charge transfer (ICT) based probes ...................................... 22

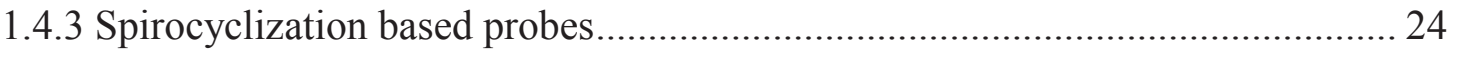

1.5 Small Fluorescent molcules as bioimaging probes .......................................... 26

1.5.1 Nitric oxide (NO) fluorescent bioimaging probes ..................................... 26

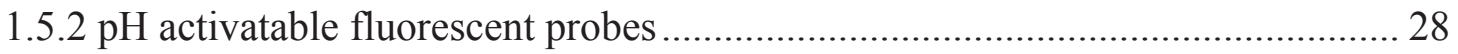

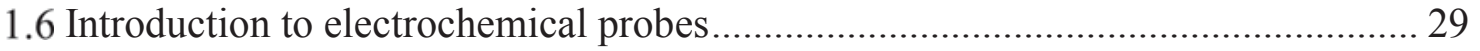

1.7 Ferrocene and polyaniline carbohydrate conjugates as biosensors....................... 30

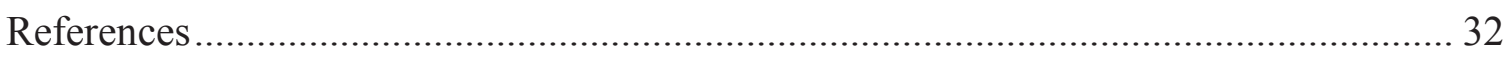

Chapter 2 : Highly Water-soluble BODIPY-based Fluorescent Probe for Sensitive and Selective Detection of Nitric Oxide in Living Cells ........................................................ 45

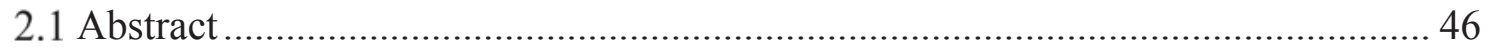

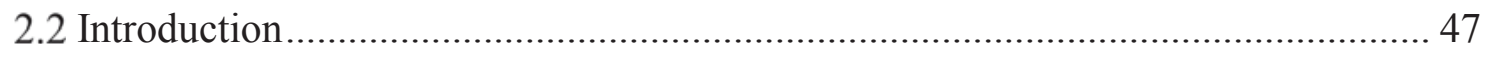




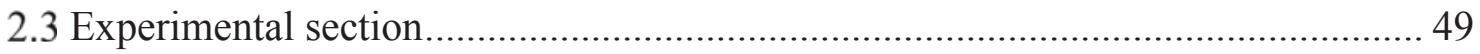

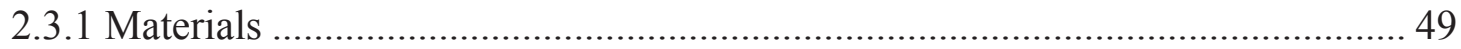

2.3.2 NOC-7 treatment and fluorescence imaging............................................. 49

2.3.3 LPS treatment and fluorescence imaging ............................................. 50

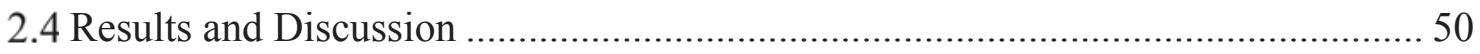

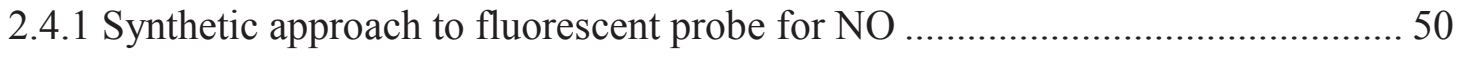

2.4.2 Optical properties of BODIPY dyes .............................................................. 51

2.4.3 The fluorescent response and selectivity of the fluorescent probe A to NO ..... 53

2.4.4 Use of fluorescent probe to detect nitric oxide in living cells ........................56

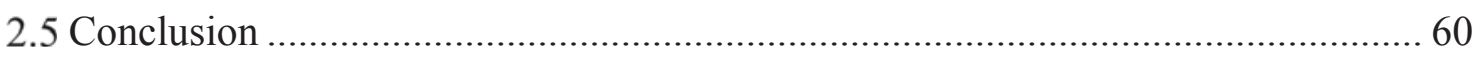

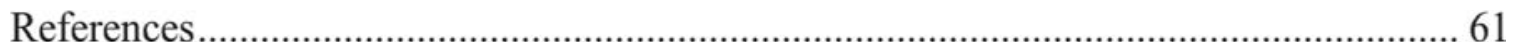

Chapter 3 : pH-Activatable Near-Infrared Fluorescent Probes for Detection of Lysosomal

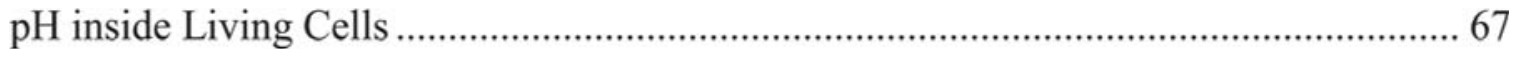

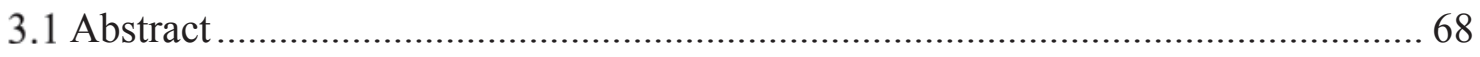

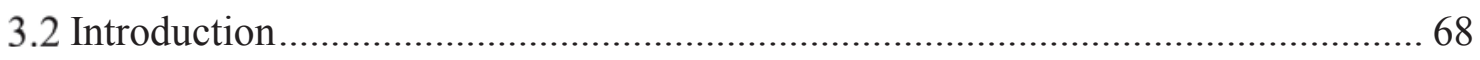

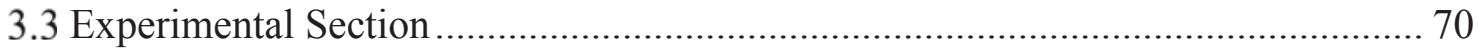

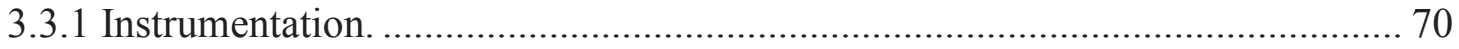

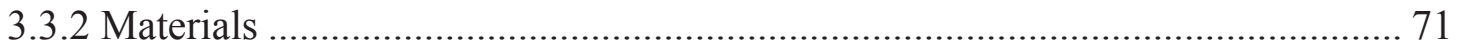

3.3.3 Cell culture and confocal fluorescence imaging ....................................... 74

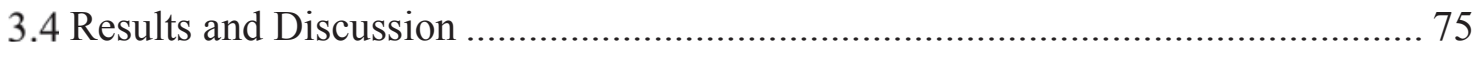

3.4.1 Design and synthesis of near-infrared fluorescent probes for $\mathrm{pH}$.................... 75

3.4.2 Optical responses of fluorescent probes to $\mathrm{pH}$.......................................... 76

3.4.3 The selectivity experiments of fluorescent probes to $\mathrm{pH}$ over metal ions......... 80

3.4.4 Live cell imaging of fluorescent probes................................................ 81 
3.5 Summary 85

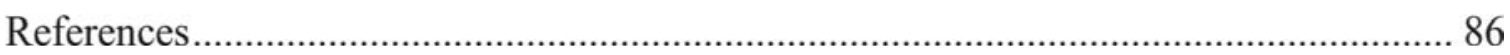

Chapter 4 : Synthesis of a Hybrid Dye, Tested its Reactivity for Fluorescent Probe Design

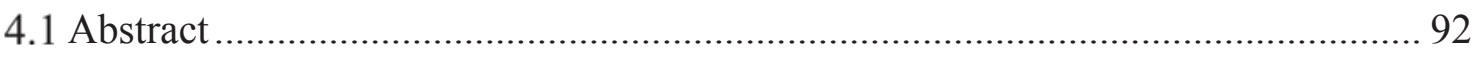

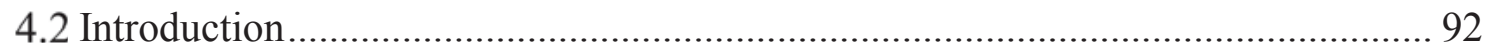

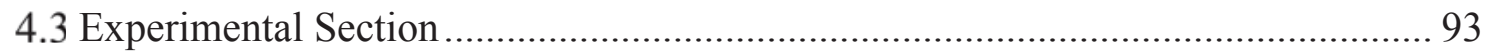

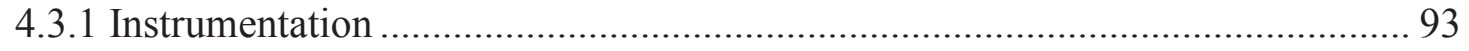

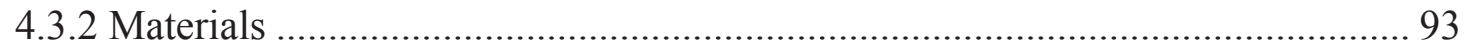

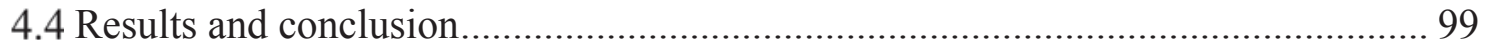

Chapter 5 : Glycosylated aniline polymer sensor: Amine to imine conversion on protein-

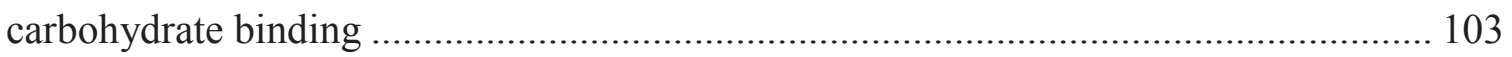

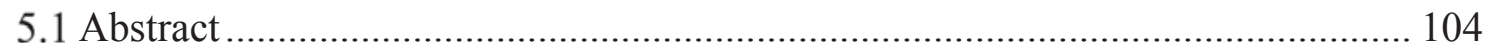

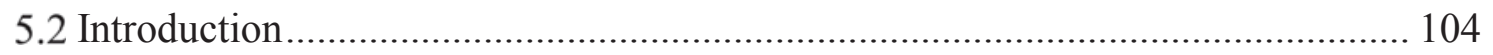

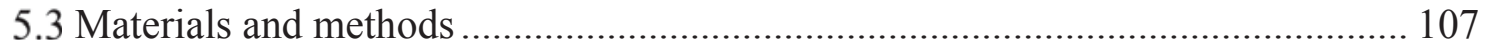

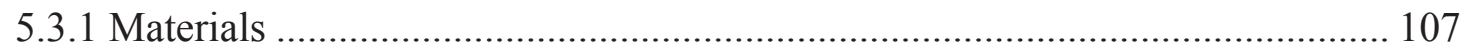

5.3.2 Synthesis and characterization of glycosylated aniline polymer film............... 107

5.3.3 Formation and characterization of mannosylated aniline polymer film ........... 111

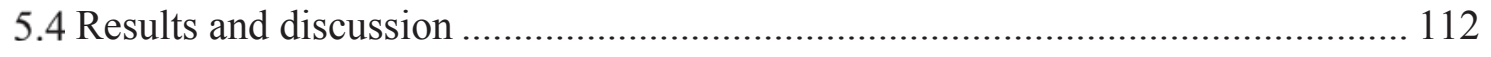

5.4.1 Characterization of mannosylated polyaniline film ........................................ 112

5.4.2 Analysis of Con A binding process by UV-vis absorption spectroscopy ........ 115

5.4.3 Characterization of the energy level of the nitrogen elements in polyaniline by XPS

5.4.4 Quantification of mannosylated polyaniline-protein interactions by electrochemical techniques for sensor application ............................................ 117 
Chapter 6 : Ferrocene-Mannose Conjugate: A Potential Electrochemical Probe for

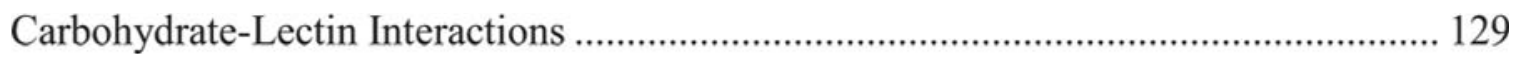

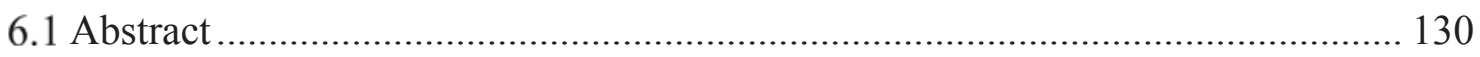

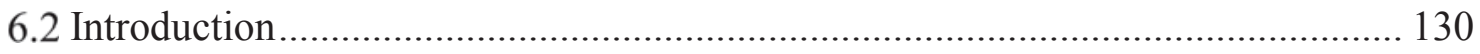

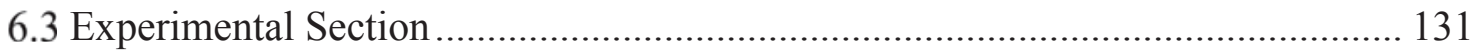

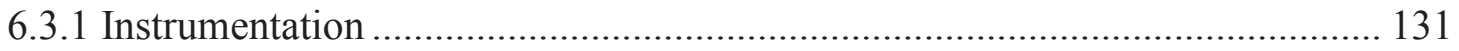

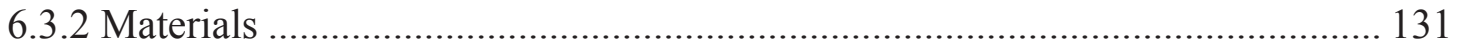

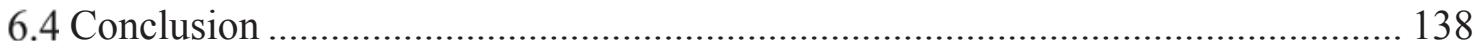

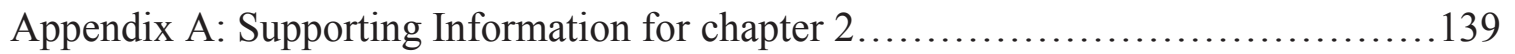

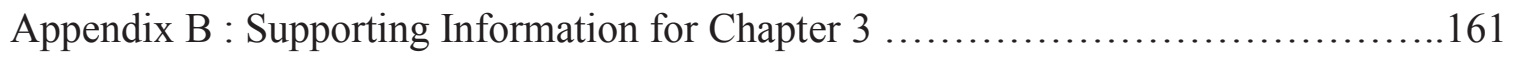

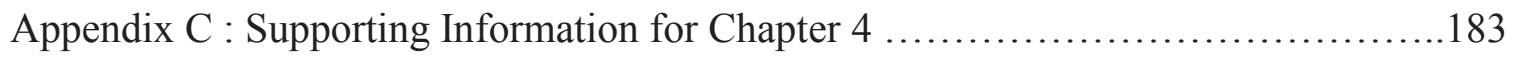

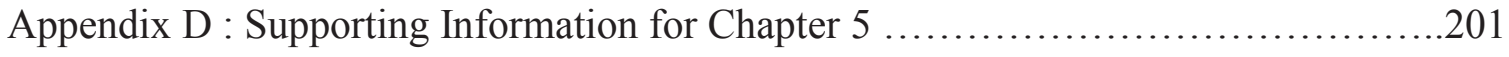

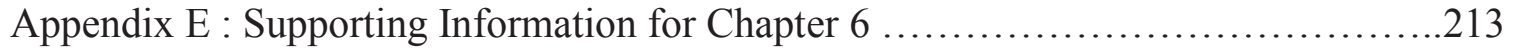

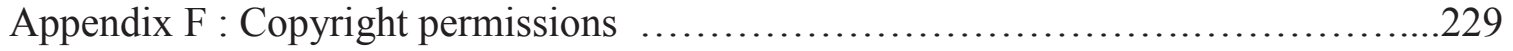




\section{PREFACE}

Chapter 2 of this dissertation was reproduced in its entirety with kind permission from ACS publications. This article was published in "ACS Appl. Mater. Interfaces, 2013, 5 (10), pp 4107-4112". This was a Multi-author paper, the author of this dissertation was responsible for the synthesis, characterization of all the reported compounds, and also for the written description of the synthesis of the reported compounds. Jingtuo Zhang collected the fluorescence data and Srinivas R. Sripathi collected the cell imaging data. Dr. Haiying Liu was the corresponding author. He conducted data analysis and revised the manuscript.

Chapter 3 is the manuscript under the review for the "Journal of Materials Chemistry B". This was a Multi-author paper, the author of this dissertation was responsible for the synthesis, characterization of all the reported compounds and also for the written description of the synthesis of the reported compounds. Jianheng Bi collected the fluorescence data and Dr. Jagadeesh Janjanam collected the cell imaging data. Dr. Haiying Liu and Dr. Ashutosh Tiwari are the corresponding authors. Dr. Haiying Liu conducted data analysis and revised the manuscript.

The material in the chapter 4 is based on the manuscript in preparation for ACS publications. The dissertation author is responsible for all the data collection. Dr. Haiying Liu will be the corresponding author. He will conduct data analysis and revision of the manuscript.

Chapter 5 of this dissertation was reproduced in its entirety with kind permission from ELSVIER publications. This article was published in "Biosensors and Bioelectronics, Volume 46, 15 August 2013, Pages 183-189". This was a Multi-author paper, the author of this dissertation was responsible for the synthesis, characterization of all the reported compounds and also for the written description of the synthesis of the reported compounds. 
Dr. Zhe Wang collected the electroanalyitcal data. Dr. Xiangqun Zeng was corresponding author. She conducted data analysis and revised the manuscript.

The material in the chapter 6 is based on the manuscript in preparation for ACS publications. The dissertation author is responsible for all the data collection. Dr. Haiying Liu will be the corresponding author. He will conduct data analysis and revision of the manuscript. 


\section{ACKNOWLEDGEMENTS}

The journey to my doctoral degree has involved countless number of people and it would be impossible to thank them all. There are few individuals that have had a direct contribution in this dissertation and it would only be appropriate to address these people here and identify their help.

I would like to express my deepest gratitude to my advisor Dr. Haiying Liu for his advice, support and patience, towards my doctoral studies. This dissertation would not have been possible without his excellent guidance and support. I would like to thank him for the time and effort spent on nurturing me as better researcher.

I would like to acknowledge my committee members Dr. Ashutosh Tiwari, Dr. Shiyue Fang and Dr. Xiaoqing Tang for their time, efforts and suggestions on my Ph.D. dissertation and defense. I would also like to thank former department chair Dr. Sarah A. Green and current department chair Dr. Cary F. Chabalowski for all the help during my $\mathrm{Ph}$.D. study. The help and understanding of all the staff of department of Chemistry which include Don Wareham, Dean Seppala, Celine Grace, Denise Laux, Margaret Dunstan, Kelly Smith, Lorri Reilly and Aparna Pandey is highly appreciated. Special thanks to Jerry Lutz for his help with instrumental needs and Organic lab supervisor Andrew Galernaue for his guidance in teaching. The financial support provided by the Department of Chemistry, MTU throughout my studies is gratefully acknowledged.

I am very much grateful to my research collaborators Dr. Wan Jin Jahng (Dept. Of Biological sciences, MTU), Dr. Ashutosh Tiwari (Dept. of Chemistry, MTU), Dr. Xiangqun Zeng (Dept. of Chemistry, OAKLAND University).

I am sincerely thankful to my lab mates and graduate students Venkat R Donuru, Sheili Zhu, Jingtuo Zhang, Jianheng Bi and others for their excellent technical assistance in the lab. 
I would like to thank all of my friends in MTU for giving me and my family memorable moments during my studies and for directly or indirectly helping me to gain knowledge. This list includes, but not limited to Venkat R Donuru, Jithendar Rao Gujja, Srinivas R. Mandalapu, Srinivas R. Sripathi, Jagadeesh Janjanam, Ramana Reddy, Rafi Shaik, and Abhilash Kantamneni.

I wish to thank my parents, in-laws, and my sister for their unconditional support and guidance throughout the years. Finally I would like to thank my wife and daughter for their constant support, understanding. Without their sacrifices and patience this work would not have been completed. 


\begin{abstract}
"Seeing is believing" the proverb well suits for fluorescent imaging probes. Since we can selectively and sensitively visualize small biomolecules, organelles such as lysosomes, neutral molecules, metal ions, anions through cellular imaging. Fluorescent probes can help shed light on the physiological and pathophysiological path ways. Since these biomolecules are produced in low concentrations in the biochemical pathways, general analytical techniques either fail to detect or are not sensitive enough to differentiate the relative concentrations. During my Ph.D. study, I exploited synthetic organic techniques to design and synthesize fluorescent probes with desirable properties such as high water solubility, high sensitivity and with varying fluorescent quantum yields. I synthesized a highly water soluble BOIDPY-based turn-on fluorescent probe for endogenous nitric oxide. I also synthesized a series of cell membrane permeable near infrared (NIR) $\mathrm{pH}$ activatable fluorescent probes for lysosomal $\mathrm{pH}$ sensing. Fluorescent dyes are molecular tools for designing fluorescent bioimaging probes. This prompted me to design and synthesize a hybrid fluorescent dye with a functionalizable chlorine atom and tested the chlorine reactivity for fluorescent probe design.
\end{abstract}

Carbohydrate and protein interactions are key for many biological processes, such as viral and bacterial infections, cell recognition and adhesion, and immune response. Among several analytical techniques aimed to study these interactions, electrochemical biosensing is more efficient due to its low cost, ease of operation, and possibility for miniaturization. During my Ph.D., I synthesized mannose bearing aniline molecule which is successfully tested as electrochemical bio sensor. A Ferrocene-mannose conjugate with an anchoring group is synthesized, which can be used as a potential electrochemical biosensor. 


\section{Chapter 1 : Introduction}

This chapter includes an introduction to subsequent chapters $(2,3,4,5$, and 6$)$ in this dissertation. This chapter covers the "Introduction to Fluorescent Probes", which covers chapters 2-4, "Introduction to Electrochemical probes", which covers chapters $5 \& 6$. The compound, figure, scheme, and equation numbers are assigned by chapter and are not continued from one chapter to another.

\subsection{Fluorescence Spectroscopy}

Photoluminescence is the emission of light from excited electronic states of the molecule created by absorption of light. Based on the nature of the excited state, photoluminescence is divided into fluorescence and phosphorescence. Fluorescence is the photoluminescence from the singlet electronic excited state. This is where electrons are in ground state and excited state are in opposite spin. Phosphorescence is also photoluminescence, but from triplet electronic excited state where electrons in ground and excited states are in the same spin. The fluorescence lifetime, the time between excitation and emission is in the order of nanoseconds. This differs for the phosphorescence lifetime, which is typically in milliseconds to seconds.

The phenomena of fluorescence was first observed, by Sir John Frederick William Herschel in 1845. In his experiment, emission of blue light was observed when quinine sulfate solution was exposed to a shorter wavelength UV light from the sun. Soon after that, in 1852, Sir George Gabriel Stokes named this phenomenon as fluorescence in the honor of the fluorescent mineral fluorspar. Stokes also discovered that emission occurs at longer wavelengths than absorption, which bears his name 'Stokes Shift'. Molecular processes involved in single fluorescent event were illustrated by Alexander Jablonski, which is famously called Jbalonski diagram (figure 1.1). These diagrams are starting point for understanding the phenomenon of fluorescence. 


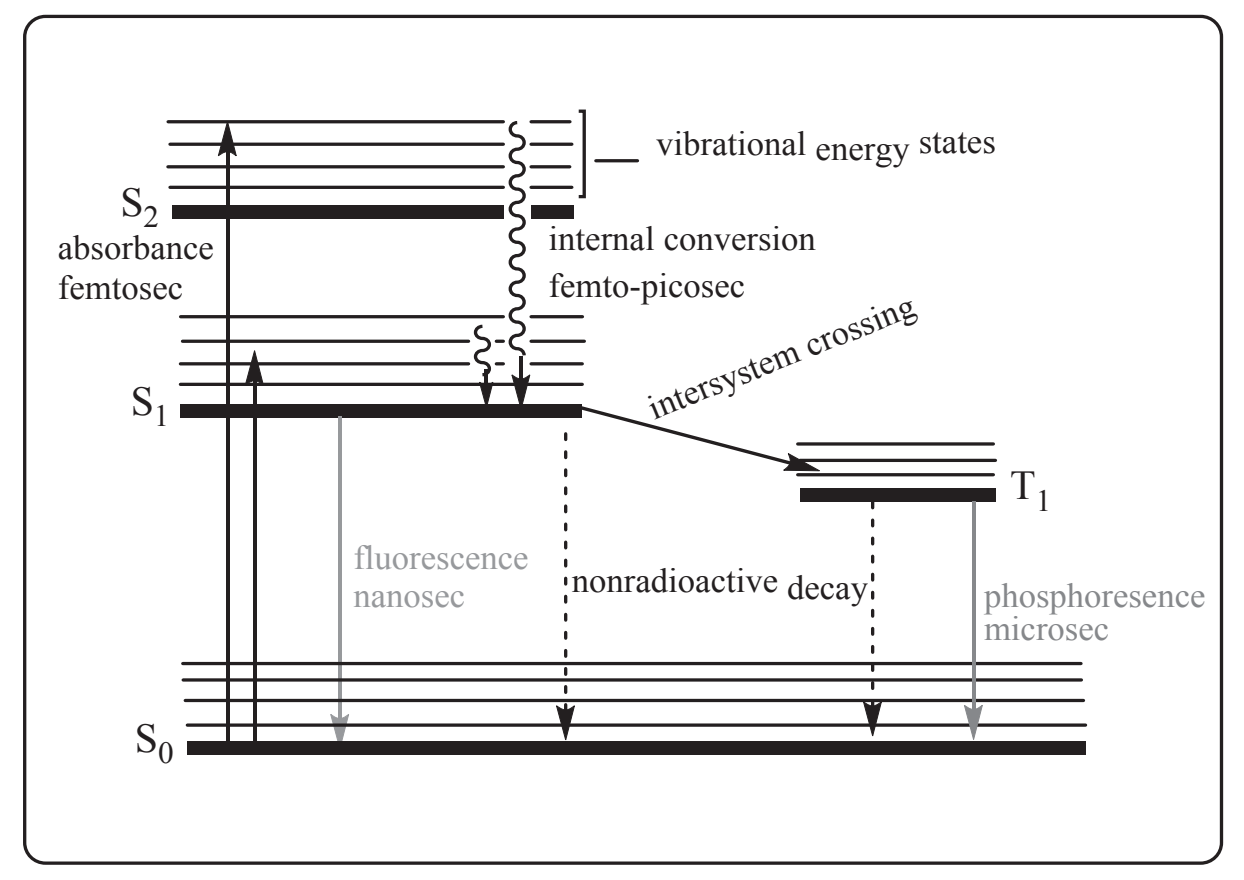

Figure 1.1 Jablonski diagram explaining the phenomena of photoluminescence

Molecules that exhibit fluorescence are called fluorophores or fluorescent molecules. Important characteristics to describe the fluophore include: absorption maxima, emission maxima, molar extinction coefficient $(\varepsilon)$, quantum yield $(\Phi)$, and lifetime $(\tau)$. Absorption and emission maxima are the maximum absorption and emission wavelengths of the fluorophore respectively. The molar extinction coefficient is the ability of the molecule's light absorption, which governs the fluorescence property of a molecule. Quantum yield, a measure for the emission efficiency of the fluorophore, is the ratio of number of photons emitted through fluorescence to the number of photons absorbed. High quantum yield is a desirable property for all fluorescence spectroscopy applications. Brightness of the fluorescent molecule, which depends on the extinction coefficient and quantum yield, is also an important criteria for fluorescent imaging applications. Fluorescent lifetime is the average time a molecule spends in its excited state before it reaches ground state through photon emission. This is another optical property that can also be considered for practical applications. 


\subsection{Small molecule fluorophores for labelling biomolecules}

Fluorescent labelling, in contrast to the absorbance-based dye or radioactive labelling, is one of the most informative and sensitive bioanalytical techniques for studying the biomolecules. Although some of the biomolecules like aromatic amino acids, NADH, Flavins are intrinsic fluorophores, using organic fluorescent dyes for labelling provides better sensitivity and visualization. Moreover the availability of fluorophores in wide a range of wavelengths and notable advancement in fluorescence instrumentation makes this area more promising. Fluorescent dyes can be attached either by covalent or noncovalent linkages, and/or using antibodies to biomolecules. The biomolecules that are visualized mainly are amino acids, peptides, proteins, and DNA. Fine tuning of fluorescent dyes for water solubility, photo stability, high quantum yields, extended conjugation, and incorporation of the variety of reactive functional groups based on the analyte is achieved by organic synthetic techniques.

For labelling from near ultraviolet to $500 \mathrm{~nm}$ wavelength range, the fluorescent dyes used are oxobenzopyrans, benzooxdiazoles, dansyl chloride, naphthalene, and 2,3dicarboxyaldehyde ${ }^{1}$. From the $500 \mathrm{~nm}$ to near-infrared region Fluorescein, Rhodamine, BODIPY, suqarines and cyanines are the majorly used fluorescent dye classes for bio labelling. Dansyl chloride is the first fluorescent molecule used to label the proteins ${ }^{2}$. For labelling biomolecules in the emission range up to $500 \mathrm{~nm}$, fluorophore derivatives based on coumarins, oligothiophenes, phanquinones, and benzooxadiazoles are reported ${ }^{3-12}$. Naphthalene based fluorophores ${ }^{13-14}$ are also used for bimolecular labelling in the emission range below $500 \mathrm{~nm}$. In the wavelength range $500-700 \mathrm{~nm}$, where problems of auto fluorescence from biomolecules are minimal, fluorescent dyes fluorescein ${ }^{15-20}$, rhodamine ${ }^{21-25}$, and BODIPY ${ }^{26-30}$ are the good choices for bimolecular labelling. In contrast to the other fluorescent dyes, above listed dyes have high molar extinction coefficients, high quantum yield, and ease of synthesis of water soluble derivatives. That attract the researchers to use these as labelling dyes. Squaraines ${ }^{31-33}$ and cynine derivatives ${ }^{34-38}$ are used as fluorescent labelling dyes in the near infrared (NIR) region. These NIR dyes are mainly used in DNA sequencing and gene sequencing applications. 


\subsection{Small molecule fluorescent probes}

In order to understand key recognition events at the molecular level in chemistry, cellular biology, and environmental sciences, it requires highly sensitive transduction (signaling) mechanisms ${ }^{39}$. Among several analytical techniques, fluorescence spectroscopy can transduce these events with sensitivity down to a single molecule level with a fast response time through easily operable and inexpensive instrumentation. Fluorescent probes are the molecules that undergo changes in photophyiscal properties of the fluorophores such as emission intensity, life-time, excitation, emission, and quantum yield with concominant environmental changes around the fluorophore through a binding event. The main components of the probes are receptor, where selective analyte binding takes place, and fluorophore where detectable spectroscopic changes occur. The performance of the fluorescent probe depends on the selectivity of the receptor to a particular analyte in a complex system and corresponding modulation of spectral properties of the fluorophore to that event.

Tsien et al. synthesized the first fluorescent probes for calcium ion ${ }^{40-41}$ using 1, 2-bis(oaminophenoxy)ethane-N,N,-N',N'-tetraacetic acid (BAPTA) as a receptor, a calcium ion chelator, in 1980. Since then, this flexible design strategy captivated the attention of researchers, which led to explosive progress in the development of fluorescent sensors for various targets. Yet there is enormous demand for highly sensitive fluorescent sensors for a variety of targets. In contrast to the conventional analytical methods, synthetic organic techniques provide the tools for flexible synthesis of fluorophore and receptor of the fluorescent probe for a target analyte. So far, fluorescent probes are prepared for a variety of anlytes that include metal cations ${ }^{42-46}$, anions ${ }^{47-49}$, reactive oxygen species ${ }^{50-53}$, reactive nitrogen species ${ }^{54-56}$, reactive sulphur species ${ }^{57-59}$, and amino acids ${ }^{60-61}$. Fluorescent probes for toxic metals ${ }^{62-64}$ and $\mathrm{pH}$ moniotinng ${ }^{65-69}$ are also synthesized. The flip side in this probe design is that extensive trial and error experimentation is needed. This should be replaced with rational design strategies that use computer simulation techniques. 


\subsection{Design principles based on different fluorescent modulation mechanisms}

In addition to the receptor binding interaction, the way of attachment of receptor and fluorophore, which are important components of the fluorescent probe system, is an important requirement for designing the fluorescent probe. Three main strategies ${ }^{70}$ for the design of fluorescent probes include 1) integration of the receptor into the $\pi$-electronic system of the fluorophore, i.e, both are in the same plane 2) linking the fluorophore and receptor with an approximately $90^{\circ}$ dihedral angle where the $\pi$-electron conjugation between them is very low 3) fluophore and receptor are covalently linked through a spacer where there is no $\pi$-electron conjugation between them (figure 1.2).

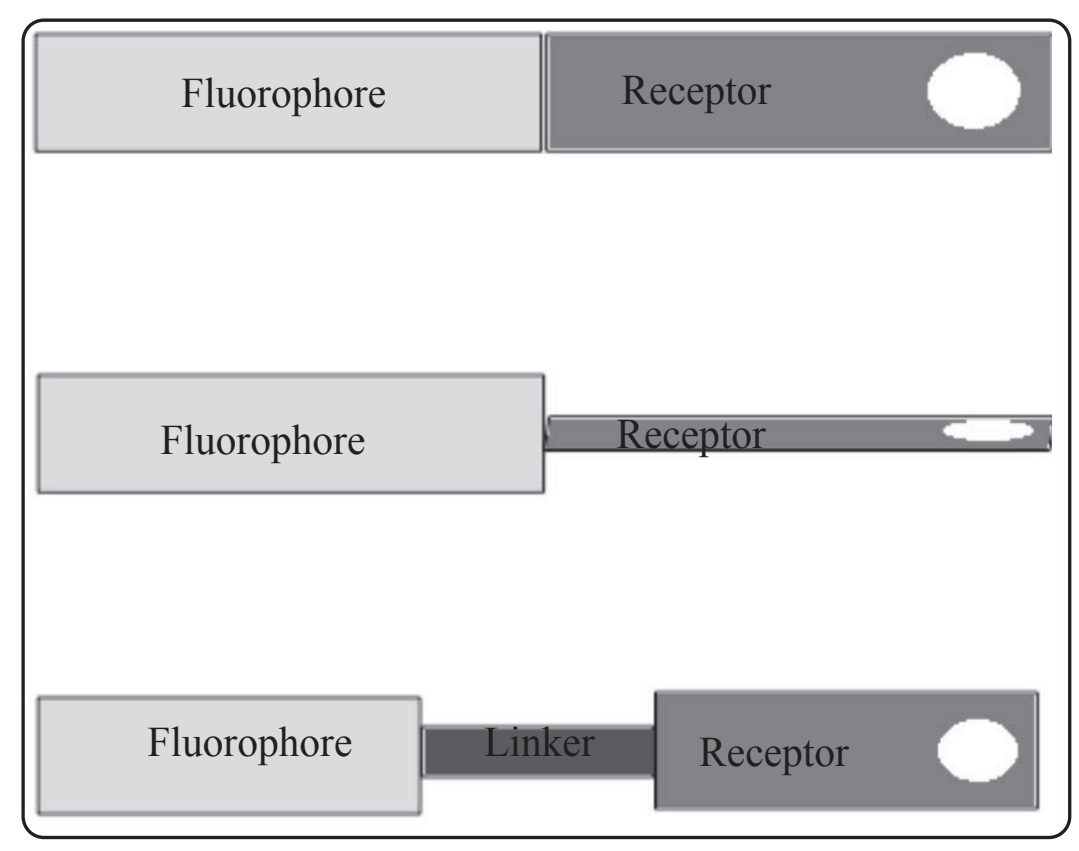

Figure 1.2. Different ways of connecting the fluorophore and receptor

Based on these design strategies, three different electronic mechanism based probes that controls the photophysical properties of the fluorophores, are reported in literature.

\subsubsection{Photo induced electron transfer (PET) based probes}

In the PET based probes, fluorescence quenching occurs when the fluorophore and receptor are linked, orthogonally or through a spacer, with an available lone pair or strong electron withdrawing group on the receptor. The regaining of fluorescence occurs when analyte 
binds to the receptor results in inhibiting the electron transfer to or from the excited fluorophore $^{71}$. It can be better explained using the frontier orbitals energies of the fluorophore and receptor. In general, a fluorophore fluoresce, when a photoexcited electron jumps to HOMO (highest occupied molecular orbital) from the LUMO (lowest unoccupied molecular orbital) with a subsequent photon emission. In PET based probes, Fluorescence is deactivated, if the energy of the lone pair orbital or the empty orbital of the receptor lies in between those of frontier orbitals of the fluorophore. This deactivation happens through blocking the deexcitation of the photoexcited electron and subsequent transfer of either receptor's lone pair electron to the hole of the fluorophore's HOMO or the fluorophore's excited electron to the receptor's empty LUMO orbital (figure 1.3). Based on the presence of available filled orbital or strong electron withdrawing group on the receptor, PET probes are divided into two types. Nagano et al. well studied and rationalize the two PET based mechanisms $^{72} 73$.

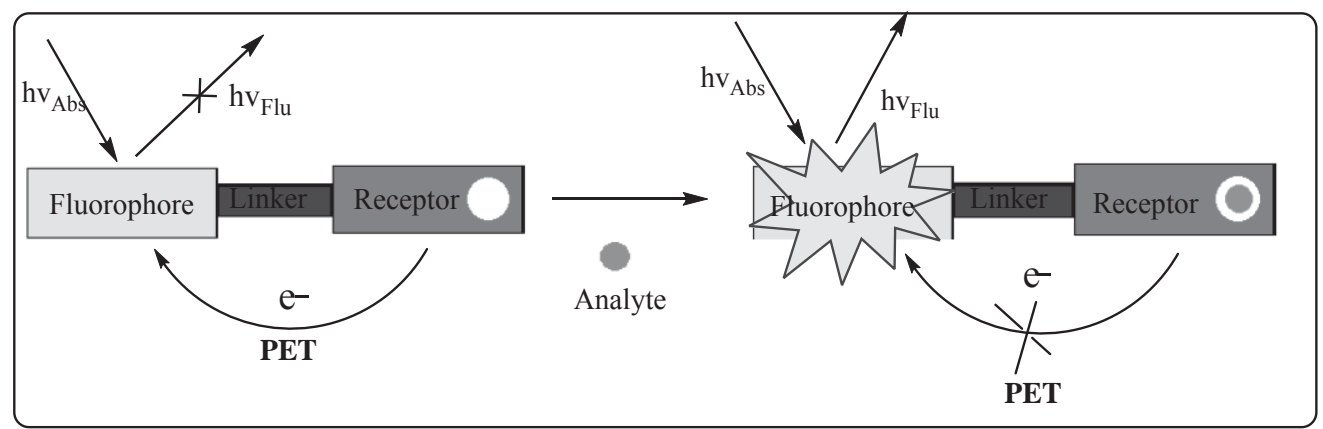

Figure 1.3. General principle of photoinduced electron transfer (PET) fluorescence mechanism

\subsubsection{Acceptor-excited photoinduced electron transfer (a-PET) based probes}

In these fluorescent modulation probes, Fluorescence is quenched when the lone pair electron transferred from the donor receptor to the HOMO of the acceptor fluorophore. This happens only if the HOMO energy of the receptor is in between those of the fluorophore frontier orbitals. In other words, oxidation potential of the receptor can be finetuned using electron donating groups to quench the fluorescence of the fluorophore. After 
a complexation or reaction with analyte through the lone pair bonding interaction, HOMO energy levels of the receptor is lowered concomitant fluorescence enhancement occurs (figure 1.4). This is a good design strategy for the "off-on" fluorescent probes synthesis. Several a-PET based fluorescent probes are reported in literature for variety of analytes ${ }^{73-}$ 82.

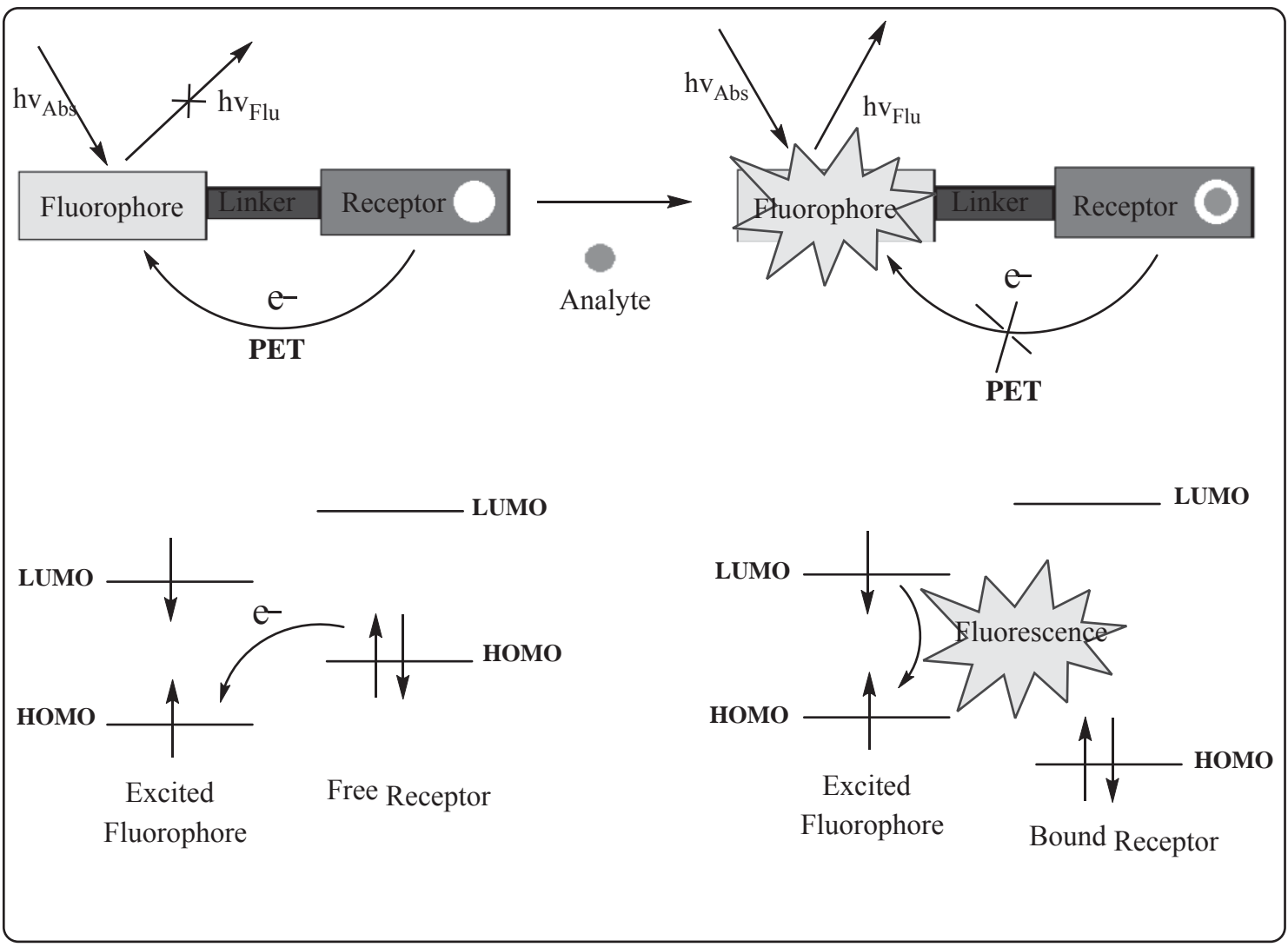

Figure 1.4. General principle, frontier orbitals energy diagram of acceptor-excited photoinduced electron transfer (a-PET) fluorescent probes

\subsubsection{Donor-excited photoinduced electron transfer (d-PET) based probes}

In these probes, fluorescence is quenched when the excited electron from the donor fluorophore is transferred to the empty LUMO orbital of the receptor. This is due to the energy of the empty LUMO of the receptor is in between those of fluorophore frontier 
orbitals (figure 1.5). In other words, fluorescence is controlled by altering the reduction potential of the receptor using different electron withdrawing groups. After the complexation with analyte, LUMO of the receptor is high enough to regain the fluorescence (figure 1.5). The feasibility of electron transfer from excited fluorophore to the receptor can be calculated using Rehm-Weller equation: $\Delta G_{e T}=E_{O X}-E_{\text {red }}-\Delta E_{0,0}-\omega_{p}$

$\Delta G_{e T}$ - Change in free energy, $E_{O X}$ - Oxidation potential of fluorophore

$E_{\text {red }}$ - Reduction potential of receptor, $\Delta E_{0,0}$ - Singlet excited state energy,

$\omega_{p}$ - work term for the charge separation state.

Equation 1.1 Rehm-Weller equation

Though only few d-PET based probes are reported in literature, this is can be a potential designing route for the development fluorescent probes for biomolecules ${ }^{56,72,83-84}$. 


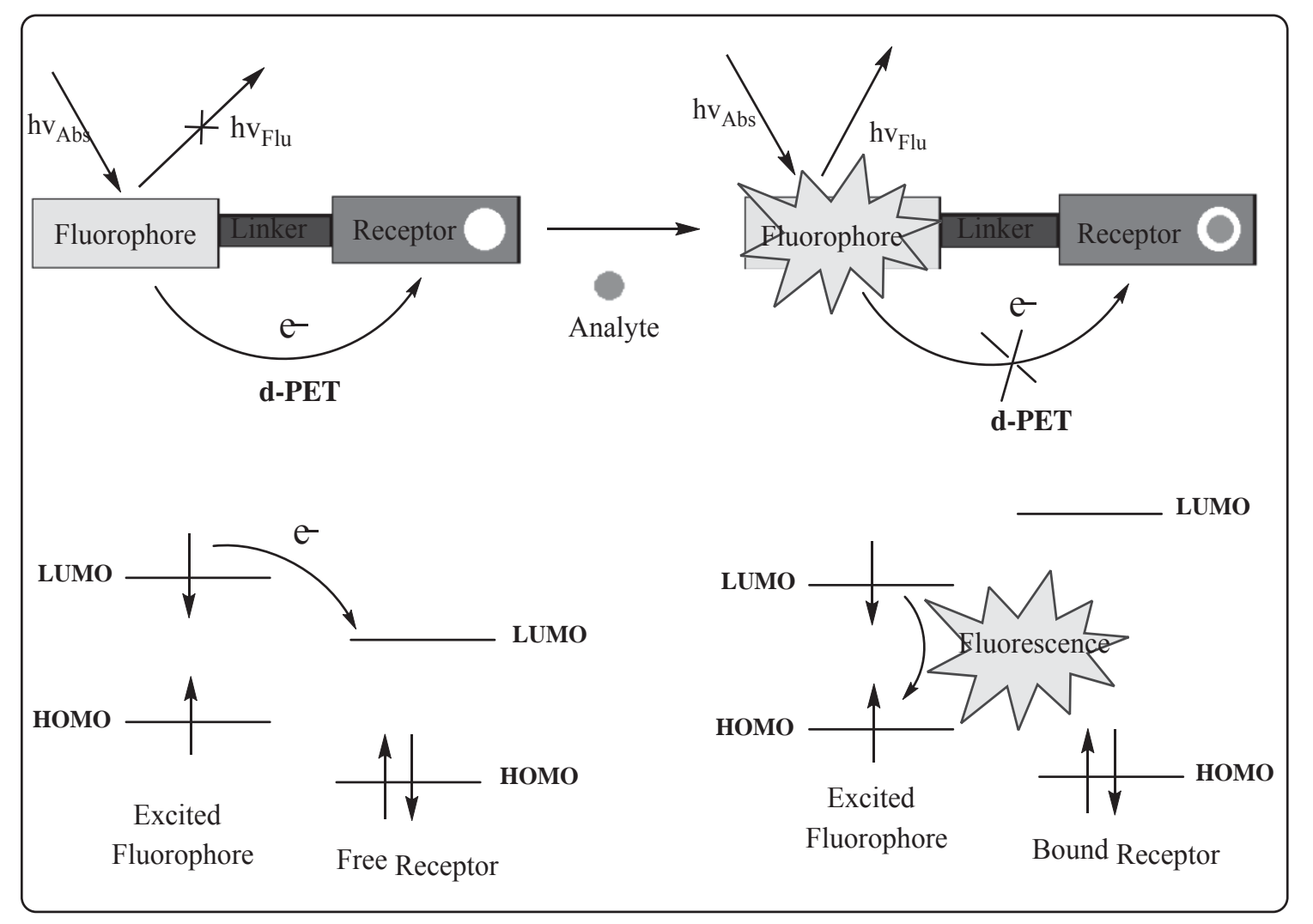

Figure 1.5. General principle, frontier orbitals energy diagram of donor-excited photoinduced electron transfer (d-PET) fluorescent probes

\subsubsection{Intramolecular charge transfer (ICT) based probes}

In these probes, both electron donating (D) and electron acceptor groups (A) are present in the Fluorophore and receptor integrated through the $\pi$-conjugated system. Thus the donor$\pi$ conjugated bridge- acceptor (D- $\pi-A)$ system is generated with a predominant intra molecular charge transfer from donor to acceptor at the excited state. Complexation or bonding of analyte either to the donor (D) or to the acceptor (A) disrupts this charge transfer in D- $\pi$-A conjugated system ${ }^{71}$. This disruption effects the electron donating or electron accepting tendency which alters the photophysical properties of the fluorophore. In other words if analyte binding causes reduction in electron donating tendencies, a blue shift in the absorption and emission spectrum of the fluorophore occurs (figure 1.6), whereas if analyte binding causes enhancement of electron acceptor tendencies, a red shift in the 
absorption and emission spectrum of the flurophore occurs (figure 1.7). To date, most fluorescent probes reported in literature for different anlytes are ICT based probes ${ }^{85-90}$ only.

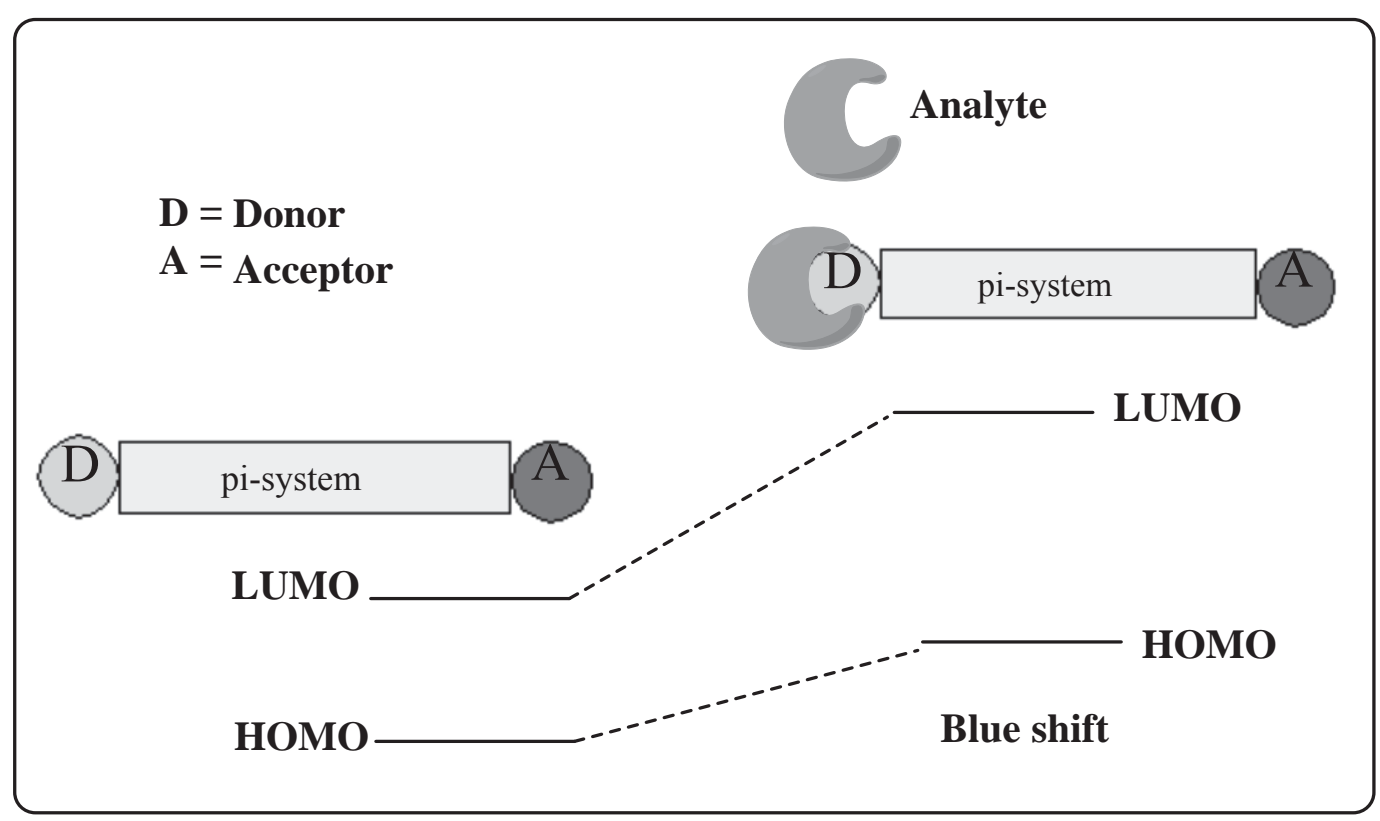

Figure 1.6. Analyte binding to donor induced fluorescent blue shift in intramolecular charge trnasfer (ICT) fluorescent probes 


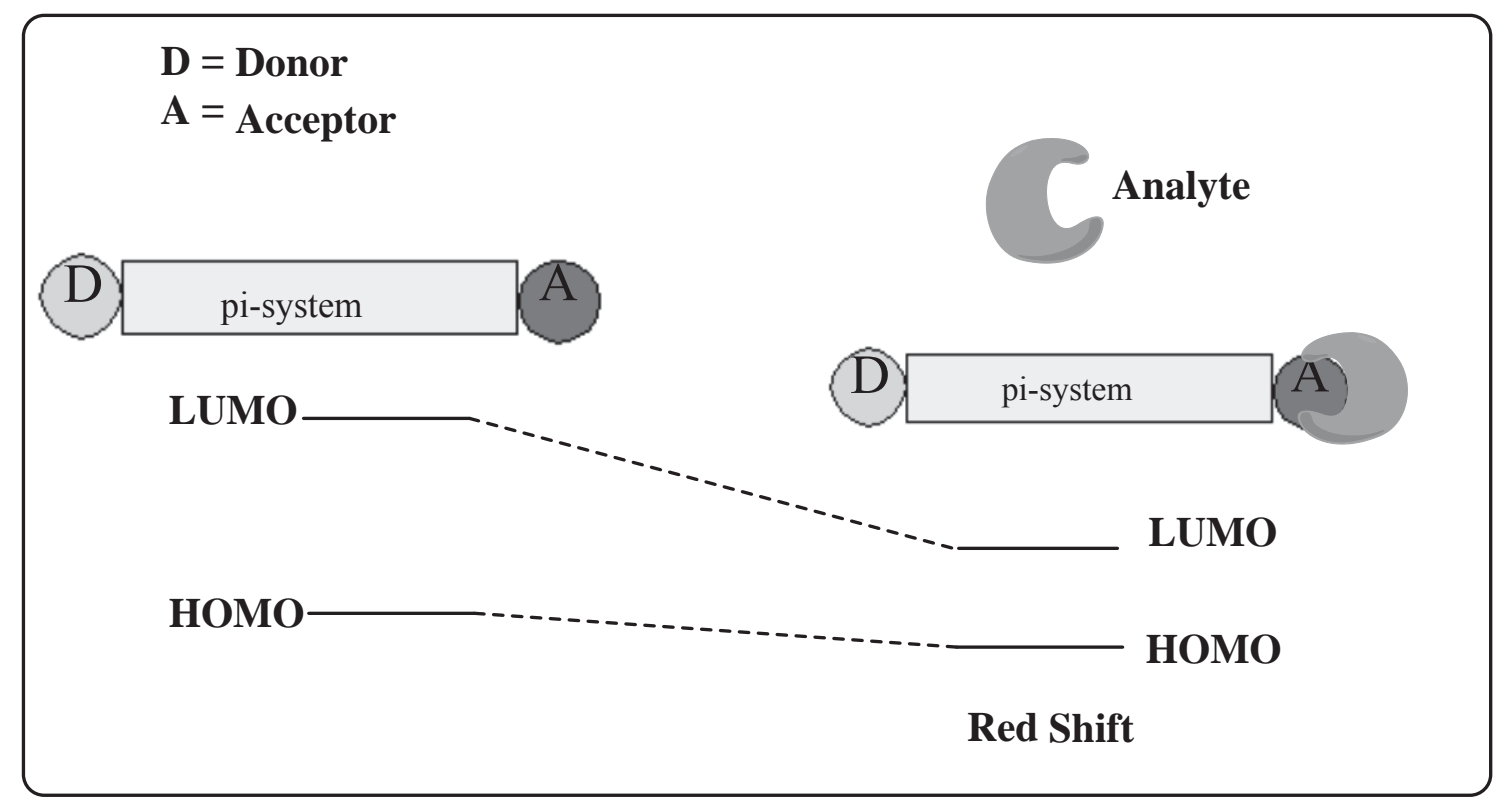

Figure 1.7. Analyte binding to acceptor induced fluorescent red shift in intramolecular charge transfer (ICT) fluorescent probes

\subsubsection{Spirocyclization based probes}

Among the fluorescent molecules, xanthone derivatives particularly rhodamines and fluoresceins have superior photophysical properties such as high extinction coefficients, superior quantum yields, and high photostability. In addition to that, these molecules are able to form intramolecular spirocyclic ring structures which deconjugates these fluorophores. Derivatives of spirolactum/spirolactone rings of these molecules are nonfluorescent, but the open ring derivatives are highly fluorescent (figure 1.8). This ring opening generally takes place in an acidic medium through the activation of carbonyl group in the spirolactum/spirolactone ring. This peculiar modulation of photophysical properties with micro environmental changes around the fluorophore leads to the development of a rational design strategy for a new turn-on fluorescent probes. In other words, analyte specific non-fluorescent spirolactum/spirolactone derivateives are developed, which are fluorescent after binding with the analyte. Czarnik et al. developed first spirocyclic based $\mathrm{Cu}^{+2}$ fluorescent probe ${ }^{91}$. After that large numner of spirocyclic turn-on fluorescent probes 
are reported for variety of analytes that include metal ions ${ }^{92-95}$, anions ${ }^{96}$, reactive oxygen species $^{52,54,97}$ thiols $^{98}$, and for $\mathrm{pH}^{\text {changes }}{ }^{99}$ etc.

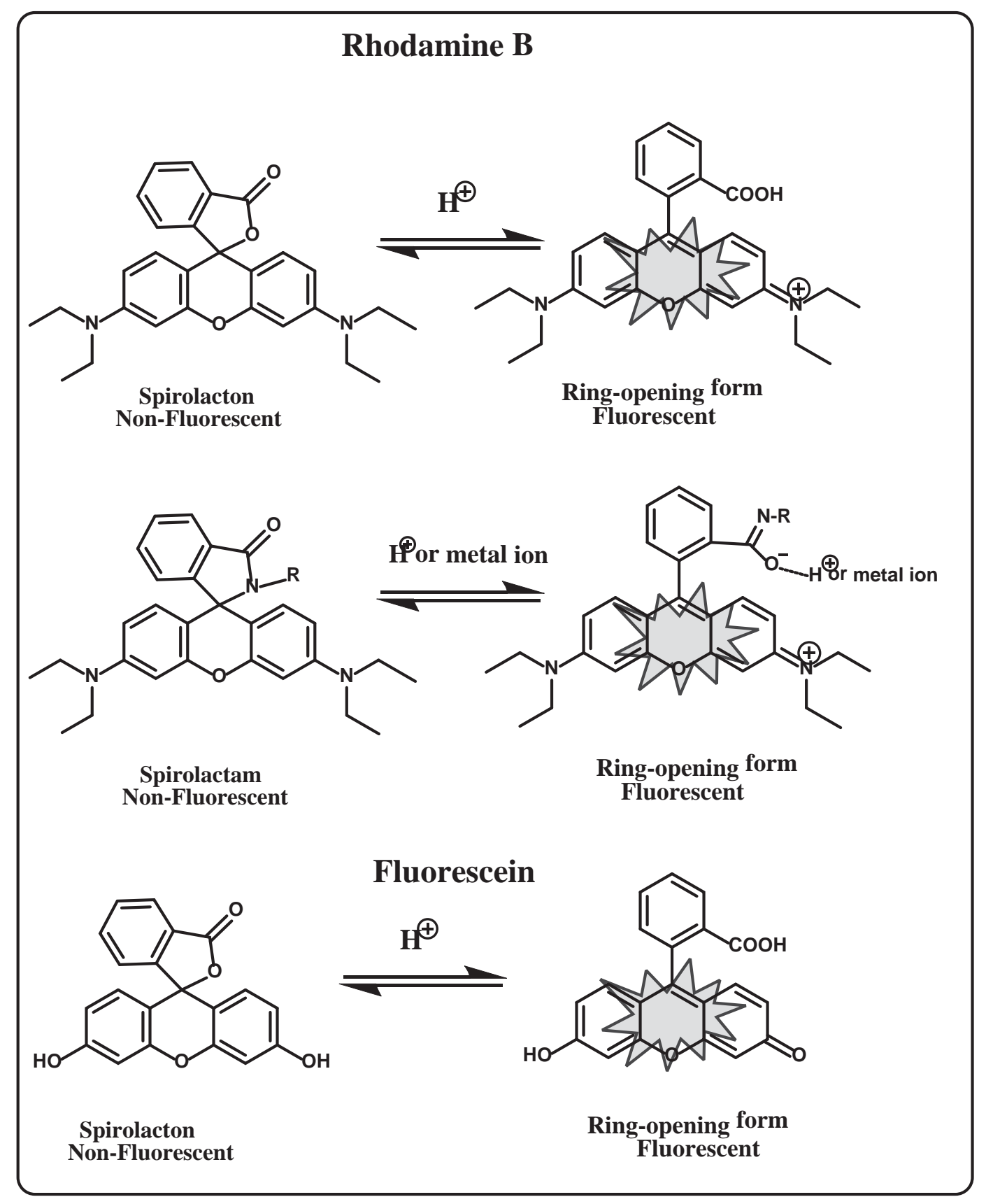

Figure 1.8. Ring-opening fluorescence turn-on mechanism in xanthene derivatives 


\subsection{Small Fluorescent molcules as bioimaging probes}

Fluorescent bioimaging, in contrast to the other expensive bioimaging techniques that includes magnetic resonance imaging, positron emission topography, and ultrasound, is highly sensitive and target specific ${ }^{100}$. Moreover, it is a less-invasive, and has a higher target to background ratio with sub cellular resolution than the conventional imaging techniques. Unlike other imaging techniques, this optical imaging technique enables the visualization of very low concentration small biomolecules such as anions, cations, amino acids, small neutral molecules in live cells, through target binding fluorescent modulation not through the accumulation in cells. The proverb "seeing is believing" is well suited for fluorescent bioimaging probes which is an indispensable tool that enables the visualization of physiological or pathophysiological changes in cells. For fluorescent bioimaging probes, synthetic chemical strategies are helpful to meet the general requirements of the probes which include longer wave lengths, brightness, non-toxic, cell membrane permeability, stability over physiological $\mathrm{pH}$ range, water solubility and photostability. A variety of small molecule fluorophores such as fluorescein, BODIPY, rhodamine and cyanine with wavelength range from blue to a near infrared region are available to synthesize tailor made imaging probes using target specific receptors. Several fluorescent bioimaging probes for very low concentration small molecule cellular analytes such as cations, anions, reactive oxygen species, and reactive nitrogen species are reported in literature.

\subsubsection{Nitric oxide (NO) fluorescent bioimaging probes}

Nitric oxide, a small reactive free radical, is a biological messenger molecule that endogenously produced by inducible and constitutive nitric oxide synthases (iNOS, cNOS). Based on its concentration it is involved in many physiological and pathophysiological processes. At low concentrations nitric oxide (NO) regulates signal transduction, smooth muscle relaxation, immune system control, neurotransmission, blood pressure control, learning and memory. In contrast mis-regulation of NO at micro molar concentrations leads to carcinogenesis, neurodegenerative disorders heart disease, hypertension, and gastrointestinal distress. Since it is highly reactive and easily diffuses through cells, detection of NO in cells is a difficult task. Although several analytical 
techniques such as spectrophotometric methods, electron paramagnetic resonance (EPR) methods, electrochemical methods, and fluorescence methods are available for NO detection, of these methods fluorescence detection is highly sensitive and amenable to imaging the intracellular and extracellular NO with high spatiotemporal resolution.

Metal based and organic reaction based are the main classes of small molecule Fluorescent probes for NO detection based on the mechanism. Metal based probes contains transition metal complex-fluorophore reporter system where fluorescence turn-on occurs either through the reduction reaction of the metal with NO or through metal-NO reaction with fluorophore displacement (figure 1.9). However the metal based probes have serious shortcomings that include cell trapability, biological incompatibility and easy leakage from the cells. Thus reaction based fluorescent probes has grabbed more attention as fluorescent bioimaging NO probes. In these probes electron rich o-phenylene diamine is used as a NO reacting site. The fluorophore-receptor system quench the fluorescence through photoinduced electron transfer (PET). After reaction with NO an electron deficient aryl triazole formation restores the fluorescene (figure 1.10). o-diaminonaphthalene (DAN), odiaminofluorescein (DAF) first commercially available NO bioimaging probes. Using this NO reacting site many NO fluorescent probes with different fluorephores like fluorescein, rhodamine, BODIPY, cyanine are reported in literature. Spirocyclization based NO probes using the same NO reacting group are also reported in the literature. However most of these NO bioimging probes are not water soluble. So highly desirable NO bioimaging probes which are cell membrane permeable, water soluble, stable at physiological $\mathrm{pH}$, biocompatible are to be synthesized. 


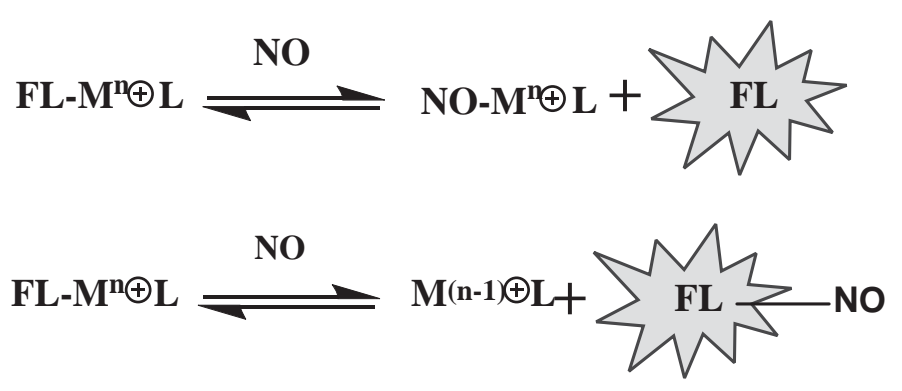

Figure 1.9. Metal based nitric oxide (NO) fluorescent probes

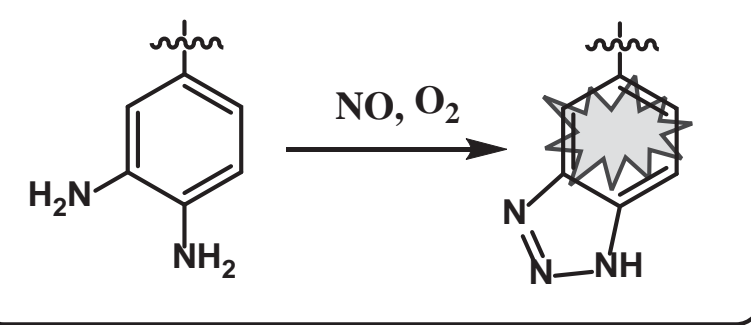

Figure 1.10. Fluorescence turn-on mechanism in nitric oxide (NO) probes

\subsection{2 $\mathrm{pH}$ activatable fluorescent probes}

Intracellular $\mathrm{pH}\left(\mathrm{pH}_{\mathrm{i}}\right)$ is an important physiological parameter that controls many cellular events including proliferation, apoptosis, ion transport, homeostasis, and cell adhesion. Aberrant cellular $\mathrm{pH}$ leads to pathological conditions that causes cancer and Alzheimer's. So, measuring the changes in cellular $\mathrm{pH}$ is highly needed to understand the intracellular pathways. Although $\mathrm{pH}$ measuring techniques like positron emission topography and magnetic resonance imaging (MRI) are available, fluorescence bioimaging probes provide more sensitivity, non-invasive to cells and high spatiotemporal resolution with real time imaging of cellular $\mathrm{pH}$ changes. Fluorescent $\mathrm{pH}$ probes mainly works on protonation/deprotonation induced fluorescent modulation mechanism (figure 1.11, 1.12). Protonation sites used in $\mathrm{pH}$ probes generally are hydroxy, carboxylic acid and amine 
groups. Also, probes can be designed based on the $\mathrm{pH}$ of the interest by tuning the $\mathrm{pKa}$ value of the $\mathrm{H}^{+}$recognition element by introducing electron donating/electron withdrawing groups. Most of the $\mathrm{pH}$ fluorescent probes synthesized so far are using xanthenes, BODIPY, and cyanine fluorophores for near neutral $\mathrm{pH}$ measurements inside the cell. Abnormal $\mathrm{pH}$ changes in acidic organelles like lysosomes are associated with pathological pathways. Since only very few liposomal $\mathrm{pH}$ probes are reported in literature, more NIR $\mathrm{pH}$ probes are needed in this regard.

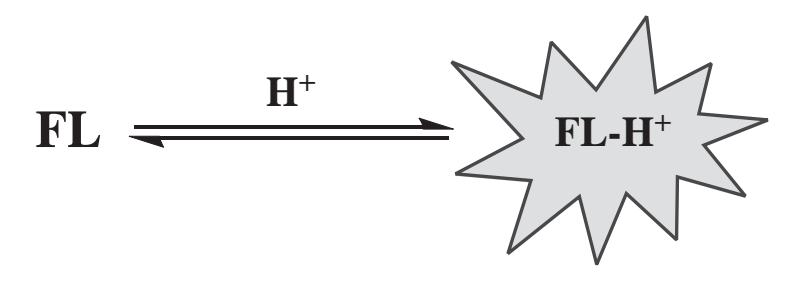

Figure 1.11. Protonation induced fluorescence activation in fluorescent $\mathrm{pH}$ probes

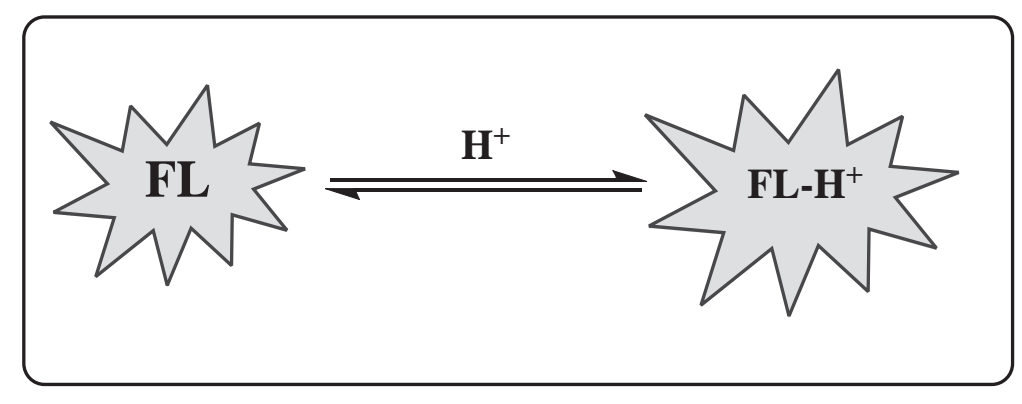

Figure 1.12. Protonation induced fluorescence shift in fluorescent $\mathrm{pH}$ probe

\subsection{Introduction to electrochemical probes}

Biological or biochemical processes at molecular level can only be understood by converting these processes into quantifiable signals. This can be achieved using a physiochemical platform that transduce the binding event into appropriate materials or devices. If the transduction of the binding event is through electrical signal those devices 
are called electrochemical probes (figure 1.13). Based on the type of measurable electrical properties, these electrochemical probes are divided into variety of models. Conductometric devices measure the binding event through altering conducting properties, potentiometric devices measure the binding event through altering potential, amperometric devices measure the binding event through altering current and impedemetric devices measure the impedence. The performance of the electrochemical probes is determined by the surface architecture that is the connectivity between the binding event and the electrode surface, and also the sensitivity of the electrodes used in the probe. When use in turbid media, high sensitivity and miniaturization prospects are some of the plus points of electrochemical sensors over others.

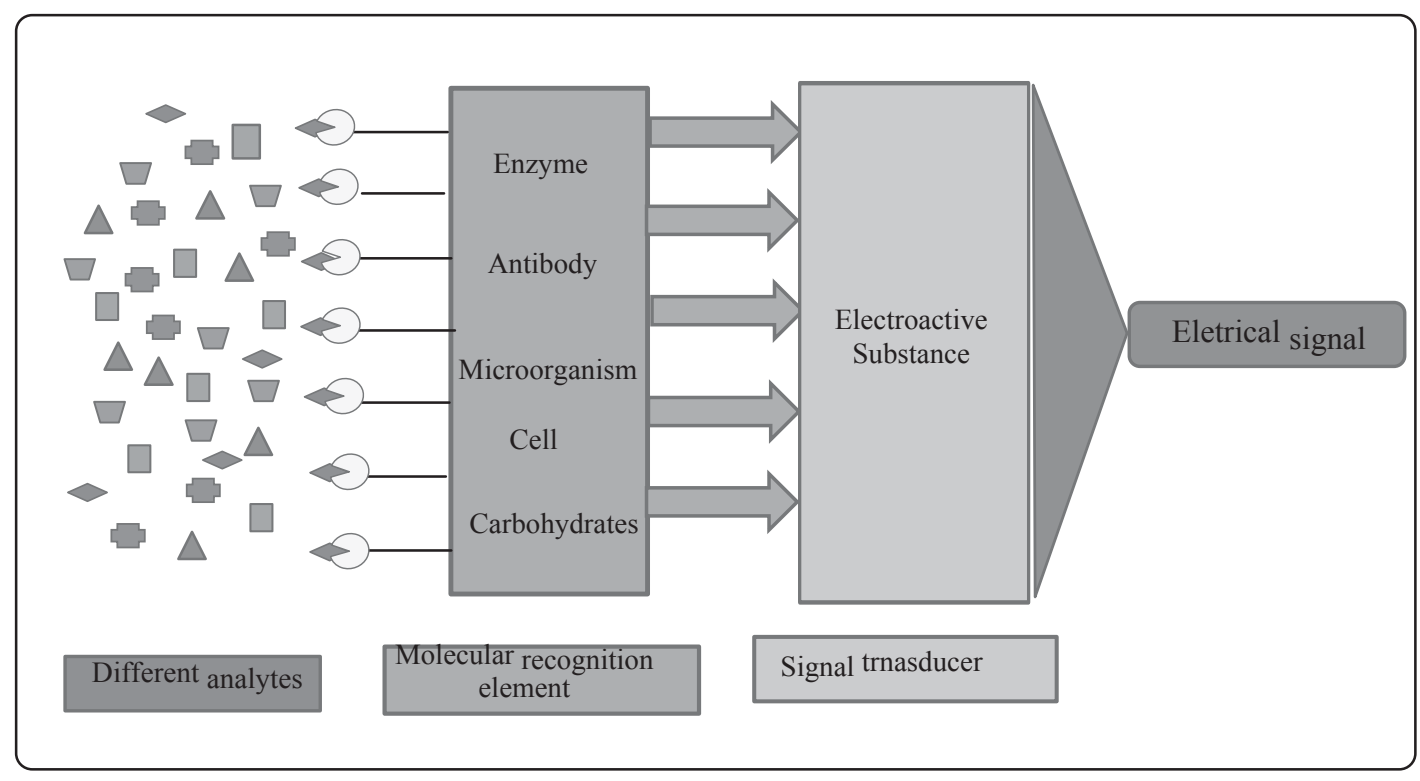

Figure 1.13. Schematic diagram for electrochemical biosensor

\subsection{Ferrocene and polyaniline carbohydrate conjugates as biosensors}

Biosensor is an analytical device that that combines the biological recognition element with a transduction mechanism. These biological recognition elements include antibodies, enzymes, proteins, carbohydrates, DNA and RNA molecules. In addition to these natural recognition elements, artificial biological recognition elements such as aptmeters, phase display antibody libraries and synthetic organic molecules are also used. Though the literature of biosensors is vast, so far only very few commercial biosensors are available. 
Glucose biosensor which is used for diabetic diagnosis, food science and biotechnology is the only commercially successful bio sensor. However continuous research of biosensors for foodborne pathogens, drug screening, environmental toxicity and genotoxicity, and so forth is going on.

Molecular interactions between the carbohydrates and cell surface proteins is crucial in understanding the many biological processes such as cell-cell adhesion, cell-growth regulation, cell-differentiation, viral and bacterial infection, immune response and cancer metastasis. In electrochemical sensors, detection of these interactions is measured as electrical signal which is triggered by the binding event. But individual carbohydrateprotein interactions are very weak. To investigate such weak interactions, amplification of those signals are necessary. This amplification is achieved through the use of synthetic multivalent carbohydrates by mimicking the nature (figure 1.14). This achieves through polymers where in each monomer is binding site, strong binding between the carbohydrates and lectins takes place through multivalent interactions on cell surface. Glyco-polymers or self-assembled monolayers (SAMs) have been employed to demonstrate that multivalent binding that occurs in biomolecules.

Poly aniline, a conjugated polymer, has been used as transduction material in electrochemical biosensors for understanding the carbohydrate-lectin interactions. The intrinsic electrical properties such as high conductivity and unique redox properties of this polymer can well transform the biological binding event into a measurable electrical signal. The polymer transport properties, multiple cooperative binding produce amplified sensitivity in sensing applications. Polyaniline's environmental stability and ease of synthesis are also additional advantages for its use as transducer. Biological binding event triggered oxidation station change in the polyaniline can be well exploited as a transduction element in the electrochemical probes.

Ferrocene's tunable redox properties and stability in aqueous, aerobic media makes it good choice as a transducer in electrochemical probes. The transducer, ferrocene, should be attached to recognition element, in this case carbohydrate, to make an electrochemical probe. Number of synthetic approaches for symmetric and asymmetric redox-active carbohydrates (glucose, mannose, and galactose) based on ferrocene have been reported. 
These redox-active carbohydrate link biological and electrical activities by transduce biological activities into electrical signals.

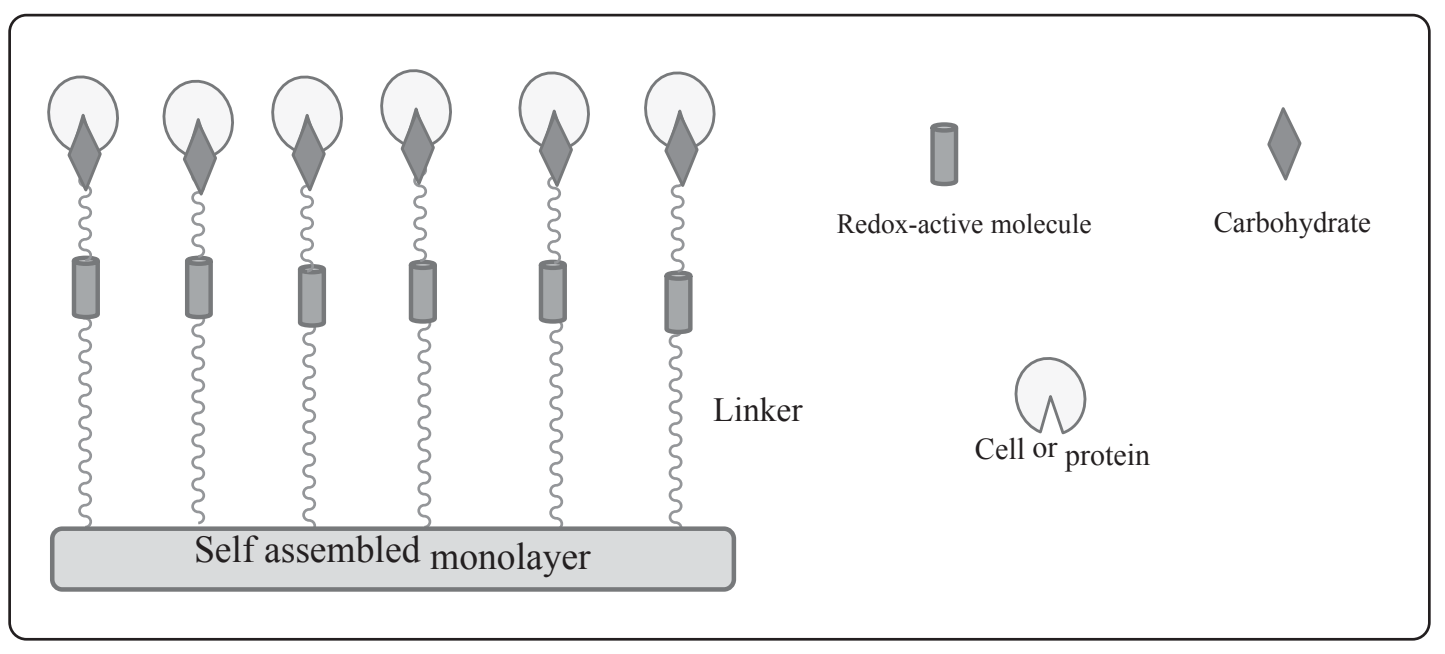

Figure 1.14. Diagram for immobilized redox active-carbohydrate binding mechanism

\section{References}

1. Gonçalves, M. S. T., Fluorescent Labeling of Biomolecules with Organic Probes. Chem Rev 2008, 109 (1), 190-212.

2. WEBER, G., Polarization of the fluorescence of macromolecules. II. Fluorescent conjugates of ovalbumin and bovine serum albumin. Biochem. J. 1952, 51 (2), 155167.

3. Berthelot, T.; Laïn, G.; Latxague, L.; Déleris, G., Synthesis of Novel Fluorogenic L-Fmoc Lysine Derivatives as Potential Tools for Imaging Cells. Journal of Fluorescence 2004, 14 (6), 671-675.

4. Han, P.; Zhou, X.; Huang, B.; Zhang, X.; Chen, C., On-gel fluorescent visualization and the site identification of S-nitrosylated proteins. Analytical Biochemistry 2008, 377 (2), 150-155. 
5. Webb, M. R.; Corrie, J. E. T., Fluorescent Coumarin-Labeled Nucleotides to Measure ADP Release from Actomyosin. Biophysical journal 2001, 81 (3), 15621569.

6. Sato, E.; Matsuhisa, A.; Sakashita, M.; Kanaoka, Y., New Water-Soluble Fluorogenic Amine. : 7-Aminocoumarin-4-methanesulfonic Acid (ACMS) and Related Substrates for Proteinases. CHEMICAL \& PHARMACEUTICAL BULLETIN 1988, 36 (9), 3496-3502.

7. Brune, M.; Hunter, J. L.; Corrie, J. E. T.; Webb, M. R., Direct, Real-Time Measurement of Rapid Inorganic Phosphate Release Using a Novel Fluorescent Probe and Its Application to Actomyosin Subfragment 1 ATPase. Biochemistry 1994, 33 (27), 8262-8271.

8. Hirshberg, M.; Henrick, K.; Lloyd Haire, L.; Vasisht, N.; Brune, M.; Corrie, J. E. T.; Webb, M. R., Crystal Structure of Phosphate Binding Protein Labeled with a Coumarin Fluorophore, a Probe for Inorganic Phosphate†, + . Biochemistry 1998, 37 (29), 10381-10385.

9. Piloto, A. M.; Rovira, D.; Costa, S. P. G.; Gonçalves, M. S. T., Oxobenzo[f]benzopyrans as new fluorescent photolabile protecting groups for the carboxylic function. Tetrahedron 2006, 62 (51), 11955-11962.

10. Capobianco, M. L.; Naldi, M.; Zambianchi, M.; Barbarella, G., Oligothiophene phosphoramidites for oligonucleotide labelling. Tetrahedron Letters 2005, 46 (47), 8181-8184.

11. Scott, D. J.; Leejeerajumnean, S.; Brannigan, J. A.; Lewis, R. J.; Wilkinson, A. J.; Hoggett, J. G., Quaternary re-arrangement analysed by spectral enhancement: the interaction of a sporulation repressor with its antagonist. Journal of Molecular Biology 1999, 293 (5), 997-1004.

12. Eugenio Vazquez, M.; Rothman, D. M.; Imperiali, B., A new environmentsensitive fluorescent amino acid for Fmoc-based solid phase peptide synthesis. Organic \& Biomolecular Chemistry 2004, 2 (14), 1965-1966. 
13. Cohen, B. E.; McAnaney, T. B.; Park, E. S.; Jan, Y. N.; Boxer, S. G.; Jan, L. Y., Probing Protein Electrostatics with a Synthetic Fluorescent Amino Acid. Science 2002, 296 (5573), 1700-1703.

14. Lu, M.-J.; Chiu, T.-C.; Chang, P.-L.; Ho, H.-T.; Chang, H.-T., Determination of glycine, glutamine, glutamate, and $\gamma$-aminobutyric acid in cerebrospinal fluids by capillary electrophoresis with light-emitting diode-induced fluorescence detection. Analytica Chimica Acta 2005, 538 (1-2), 143-150.

15. Konecsni, T.; Kilár, F., Monitoring of the conjugation reaction between human serum transferrin and fluorescein isothiocyanate by capillary electrophoresis. Journal of Chromatography A 2004, 1051 (1-2), 135-139.

16. Brimble, M. A.; Kowalczyk, R.; Harris, P. W. R.; Dunbar, P. R.; Muir, V. J., Synthesis of fluorescein-labelled O-mannosylated peptides as components for synthetic vaccines: comparison of two synthetic strategies. Organic \& Biomolecular Chemistry 2008, 6 (1), 112-121.

17. Gosalia, D. N.; Salisbury, C. M.; Maly, D. J.; Ellman, J. A.; Diamond, S. L., Profiling serine protease substrate specificity with solution phase fluorogenic peptide microarrays. PROTEOMICS 2005, 5 (5), 1292-1298.

18. Tian, M.; Wu, X.-L.; Zhang, B.; Li, J.-L.; Shi, Z., Synthesis of chlorinated fluoresceins for labeling proteins. Bioorganic \& Medicinal Chemistry Letters 2008, 18 (6), 1977-1979.

19. Griffin, B. A.; Adams, S. R.; Tsien, R. Y., Specific Covalent Labeling of Recombinant Protein Molecules Inside Live Cells. Science 1998, 281 (5374), 269272.

20. Kottegoda, S.; Aoto, P. C.; Sims, C. E.; Allbritton, N. L., Biarsenical-Tetracysteine Motif as a Fluorescent Tag for Detection in Capillary Electrophoresis. Analytical Chemistry 2008, 80 (14), 5358-5366.

21. Horneffer, V.; Forsmann, A.; Strupat, K.; Hillenkamp, F.; Kubitscheck, U., Localization of Analyte Molecules in MALDI Preparations by Confocal Laser Scanning Microscopy. Analytical Chemistry 2001, 73 (5), 1016-1022. 
22. Mandalà, M.; Serck-Hanssen, G.; Martino, G.; Helle, K. B., The Fluorescent Cationic Dye Rhodamine 6G as a Probe for Membrane Potential in Bovine Aortic Endothelial Cells. Analytical Biochemistry 1999, 274 (1), 1-6.

23. Wang, Y.; Hao, D.; Stein, W. D.; Yang, L., A kinetic study of Rhodamine123 pumping by P-glycoprotein. Biochimica et Biophysica Acta (BBA) - Biomembranes 2006, 1758 (10), 1671-1676.

24. Bandichhor, R.; Petrescu, A. D.; Vespa, A.; Kier, A. B.; Schroeder, F.; Burgess, K., Synthesis of a New Water-Soluble Rhodamine Derivative and Application to Protein Labeling and Intracellular Imaging. Bioconjugate Chemistry 2006, 17 (5), 1219-1225.

25. Gao, Y.; Li, J. L.; Shi, Z., Synthesis and application of rhodamine-based fluorescent probe dyes with spacer linker arm. Chinese Chemical Letters 2007, 18 (6), 667669.

26. Metzker, M. L.; Lu, J.; Gibbs, R. A., Electrophoretically Uniform Fluorescent Dyes for Automated DNA Sequencing. Science 1996, 271 (5254), 1420-1422.

27. Lee, L. G.; Connell, C. R.; Woo, S. L.; Cheng, R. D.; McArdle, B. F.; Fuller, C. W.; Halloran, N. D.; Wilson, R. K., DNA sequencing with dye-labeled terminators and T7 DNA polymerase: effect of dyes and dNTPs on incorporation of dyeterminators and probability analysis of termination fragments. Nucleic Acids Research 1992, 20 (10), 2471-2483.

28. Kálai, T.; Hideg, K., Synthesis of new, BODIPY-based sensors and labels. Tetrahedron 2006, 62 (44), 10352-10360.

29. Hubbell, W. L.; Altenbach, C.; Hubbell, C. M.; Khorana, H. G., Rhodopsin structure, dynamics, and activation: A perspective from crystallography, sitedirected spin labeling, sulfhydryl reactivity, and disulfide cross-linking. In Advances in Protein Chemistry, Douglas, C. R., Ed. Academic Press: 2003; Vol. Volume 63, pp 243-290.

30. Olofsson, M.; Kalinin, S.; Zdunek, J.; Oliveberg, M.; Johansson, L. B. A., Tryptophan-BODIPY: A versatile donor-acceptor pair for probing generic changes 
of intraprotein distances. Physical Chemistry Chemical Physics 2006, 8 (26), 31303140 .

31. Tatarets, A. L.; Fedyunyayeva, I. A.; Dyubko, T. S.; Povrozin, Y. A.; Doroshenko, A. O.; Terpetschnig, E. A.; Patsenker, L. D., Synthesis of water-soluble, ringsubstituted squaraine dyes and their evaluation as fluorescent probes and labels. Analytica Chimica Acta 2006, 570 (2), 214-223.

32. Volkova, K. D.; Kovalska, V. B.; Tatarets, A. L.; Patsenker, L. D.; Kryvorotenko, D. V.; Yarmoluk, S. M., Spectroscopic study of squaraines as protein-sensitive fluorescent dyes. Dyes and Pigments 2007, 72 (3), 285-292.

33. Yagi, S.; Murayama, S.; Hyodo, Y.; Fujie, Y.; Hirose, M.; Nakazumi, H., Synthesis and light absorption/emission properties of novel bis-squaraine dyes with extensively conjugated [small pi]-electron systems. Journal of the Chemical Society, Perkin Transactions 1 2002, (12), 1417-1419.

34. Gómez-Hens, A.; Aguilar-Caballos, M. P., Long-wavelength fluorophores: new trends in their analytical use. TrAC Trends in Analytical Chemistry 2004, 23 (2), 127-136.

35. Mitra, R. D.; Shendure, J.; Olejnik, J.; Edyta Krzymanska, O.; Church, G. M., Fluorescent in situ sequencing on polymerase colonies. Analytical Biochemistry 2003, $320(1), 55-65$.

36. McCorquodale, E. M.; Colyer, C. L., Indocyanine green as a noncovalent, pseudofluorogenic label for protein determination by capillary electrophoresis. ELECTROPHORESIS 2001, 22 (12), 2403-2408.

37. Hilderbrand, S. A.; Kelly, K. A.; Weissleder, R.; Tung, C.-H., Monofunctional Near-Infrared Fluorochromes for Imaging Applications. Bioconjugate Chemistry 2005, $16(5), 1275-1281$.

38. Flanagan, J. H.; Khan, S. H.; Menchen, S.; Soper, S. A.; Hammer, R. P., Functionalized Tricarbocyanine Dyes as Near-Infrared Fluorescent Probes for Biomolecules. Bioconjugate Chemistry 1997, 8 (5), 751-756. 
39. de Silva, A. P.; Gunaratne, H. Q. N.; Gunnlaugsson, T.; Huxley, A. J. M.; McCoy, C. P.; Rademacher, J. T.; Rice, T. E., Signaling Recognition Events with Fluorescent Sensors and Switches. Chem Rev 1997, 97 (5), 1515-1566.

40. Tsien, R. Y., New calcium indicators and buffers with high selectivity against magnesium and protons: design, synthesis, and properties of prototype structures. Biochemistry 1980, 19 (11), 2396-2404.

41. Grynkiewicz, G.; Poenie, M.; Tsien, R. Y., A new generation of Ca2+ indicators with greatly improved fluorescence properties. Journal of Biological Chemistry 1985, 260 (6), 3440-3450.

42. Suzuki, Y.; Komatsu, H.; Ikeda, T.; Saito, N.; Araki, S.; Citterio, D.; Hisamoto, H.; Kitamura, Y.; Kubota, T.; Nakagawa, J.; Oka, K.; Suzuki, K., Design and Synthesis of Mg2+-Selective Fluoroionophores Based on a Coumarin Derivative and Application for $\mathrm{Mg} 2+$ Measurement in a Living Cell. Analytical Chemistry 2002, 74 (6), 1423-1428.

43. Minta, A.; Tsien, R. Y., Fluorescent indicators for cytosolic sodium. Journal of Biological Chemistry 1989, 264 (32), 19449-19457.

44. Callan, J. F.; de Silva, A. P.; Magri, D. C., Luminescent sensors and switches in the early 21 st century. Tetrahedron 2005, 61 (36), 8551-8588.

45. Sen, S.; Sarkar, S.; Chattopadhyay, B.; Moirangthem, A.; Basu, A.; Dhara, K.; Chattopadhyay, P., A ratiometric fluorescent chemosensor for iron: discrimination of Fe2+ and Fe3+ and living cell application. Analyst 2012, 137 (14), 3335-3342.

46. Hirano, T.; Kikuchi, K.; Urano, Y.; Nagano, T., Improvement and Biological Applications of Fluorescent Probes for Zinc, ZnAFs. Journal of the American Chemical Society 2002, 124 (23), 6555-6562.

47. Chen, X.; Nam, S.-W.; Kim, G.-H.; Song, N.; Jeong, Y.; Shin, I.; Kim, S. K.; Kim, J.; Park, S.; Yoon, J., A near-infrared fluorescent sensor for detection of cyanide in aqueous solution and its application for bioimaging. Chemical Communications 2010, 46 (47), 8953-8955. 
48. Zhu, B.; Yuan, F.; Li, R.; Li, Y.; Wei, Q.; Ma, Z.; Du, B.; Zhang, X., A highly selective colorimetric and ratiometric fluorescent chemodosimeter for imaging fluoride ions in living cells. Chemical Communications 2011, 47 (25), 7098-7100.

49. Kumar, R.; Kumar, S.; Singh, P.; Hundal, G.; Hundal, M. S.; Kumar, S., A fluorescent chemosensor for detection of perchlorate ions in water. Analyst 2012, $137(21), 4913-4916$.

50. Maeda, H.; Fukuyasu, Y.; Yoshida, S.; Fukuda, M.; Saeki, K.; Matsuno, H.; Yamauchi, Y.; Yoshida, K.; Hirata, K.; Miyamoto, K., Fluorescent Probes for Hydrogen Peroxide Based on a Non-Oxidative Mechanism. Angewandte Chemie International Edition 2004, 43 (18), 2389-2391.

51. Maeda, H.; Yamamoto, K.; Kohno, I.; Hafsi, L.; Itoh, N.; Nakagawa, S.; Kanagawa, N.; Suzuki, K.; Uno, T., Design of a Practical Fluorescent Probe for Superoxide Based on Protection-Deprotection Chemistry of Fluoresceins with Benzenesulfonyl Protecting Groups. Chemistry - A European Journal 2007, 13 (7), 1946-1954.

52. Yapici, N. B.; Jockusch, S.; Moscatelli, A.; Mandalapu, S. R.; Itagaki, Y.; Bates, D. K.; Wiseman, S.; Gibson, K. M.; Turro, N. J.; Bi, L., New Rhodamine Nitroxide Based Fluorescent Probes for Intracellular Hydroxyl Radical Identification in Living Cells. Organic Letters 2011, 14 (1), 50-53.

53. Leslie, A. K.; Li, D.; Koide, K., Amine-Promoted $\beta$-Elimination of a $\beta$-Aryloxy Aldehyde for Fluorogenic Chemodosimeters. The Journal of Organic Chemistry 2011, 76 (16), 6860-6865.

54. Zheng, H.; Shang, G.-Q.; Yang, S.-Y.; Gao, X.; Xu, J.-G., Fluorogenic and Chromogenic Rhodamine Spirolactam Based Probe for Nitric Oxide by Spiro Ring Opening Reaction. Organic Letters 2008, 10 (12), 2357-2360.

55. Yang, D.; Wang, H.-L.; Sun, Z.-N.; Chung, N.-W.; Shen, J.-G., A Highly Selective Fluorescent Probe for the Detection and Imaging of Peroxynitrite in Living Cells. Journal of the American Chemical Society 2006, 128 (18), 6004-6005. 
56. Ueno, T.; Urano, Y.; Kojima, H.; Nagano, T., Mechanism-Based Molecular Design of Highly Selective Fluorescence Probes for Nitrative Stress. Journal of the American Chemical Society 2006, 128 (33), 10640-10641.

57. Ros-Lis, J. V.; García, B.; Jiménez, D.; Martínez-Máñez, R.; Sancenón, F.; Soto, J.; Gonzalvo, F.; Valldecabres, M. C., Squaraines as Fluoro-Chromogenic Probes for Thiol-Containing Compounds and Their Application to the Detection of Biorelevant Thiols. Journal of the American Chemical Society 2004, 126 (13), 4064-4065.

58. Zhu, B.; Zhang, X.; Li, Y.; Wang, P.; Zhang, H.; Zhuang, X., A colorimetric and ratiometric fluorescent probe for thiols and its bioimaging applications. Chemical Communications 2010, 46 (31), 5710-5712.

59. Lippert, A. R.; New, E. J.; Chang, C. J., Reaction-Based Fluorescent Probes for Selective Imaging of Hydrogen Sulfide in Living Cells. Journal of the American Chemical Society 2011, 133 (26), 10078-10080.

60. Long, L.; Lin, W.; Chen, B.; Gao, W.; Yuan, L., Construction of a FRET-based ratiometric fluorescent thiol probe. Chemical Communications 2011, 47 (3), 893895.

61. Sun, Y.-Q.; Chen, M.; Liu, J.; Lv, X.; Li, J.-f.; Guo, W., Nitroolefin-based coumarin as a colorimetric and fluorescent dual probe for biothiols. Chemical Communications 2011, 47 (39), 11029-11031.

62. Kwon, J. Y.; Jang, Y. J.; Lee, Y. J.; Kim, K. M.; Seo, M. S.; Nam, W.; Yoon, J., A Highly Selective Fluorescent Chemosensor for Pb2+. Journal of the American Chemical Society 2005, 127 (28), 10107-10111.

63. Peng, X.; Du, J.; Fan, J.; Wang, J.; Wu, Y.; Zhao, J.; Sun, S.; Xu, T., A Selective Fluorescent Sensor for Imaging $\mathrm{Cd} 2+$ in Living Cells. Journal of the American Chemical Society 2007, 129 (6), 1500-1501.

64. Lin, W.; Cao, X.; Ding, Y.; Yuan, L.; Long, L., A highly selective and sensitive fluorescent probe for $\mathrm{Hg} 2+$ imaging in live cells based on a rhodamine-thioamidealkyne scaffold. Chemical Communications 2010, 46 (20), 3529-3531. 
65. Rink, T. J.; Tsien, R. Y.; Pozzan, T., Cytoplasmic $\mathrm{pH}$ and free $\mathrm{Mg} 2+$ in lymphocytes. The Journal of Cell Biology 1982, 95 (1), 189-196.

66. Han, J.; Loudet, A.; Barhoumi, R.; Burghardt, R. C.; Burgess, K., A Ratiometric pH Reporter For Imaging Protein-dye Conjugates In Living Cells. Journal of the American Chemical Society 2009, 131 (5), 1642-1643.

67. Chen, S.; Liu, J.; Liu, Y.; Su, H.; Hong, Y.; Jim, C. K. W.; Kwok, R. T. K.; Zhao, N.; Qin, W.; Lam, J. W. Y.; Wong, K. S.; Tang, B. Z., An AIE-active hemicyanine fluorogen with stimuli-responsive red/blue emission: extending the $\mathrm{pH}$ sensing range by "switch + knob" effect. Chemical Science 2012, 3 (6), 1804-1809.

68. Tang, B.; Liu, X.; Xu, K.; Huang, H.; Yang, G.; An, L., A dual near-infrared pH fluorescent probe and its application in imaging of HepG2 cells. Chemical Communications 2007, (36), 3726-3728.

69. Myochin, T.; Kiyose, K.; Hanaoka, K.; Kojima, H.; Terai, T.; Nagano, T., Rational Design of Ratiometric Near-Infrared Fluorescent $\mathrm{pH}$ Probes with Various pKa Values, Based on Aminocyanine. Journal of the American Chemical Society 2011, 133 (10), 3401-3409.

70. Basabe-Desmonts, L.; Reinhoudt, D. N.; Crego-Calama, M., Design of fluorescent materials for chemical sensing. Chemical Society Reviews 2007, 36 (6), 993-1017.

71. Yang, Y.; Zhao, Q.; Feng, W.; Li, F., Luminescent Chemodosimeters for Bioimaging. Chem Rev 2012, 113 (1), 192-270.

72. Ueno, T.; Urano, Y.; Setsukinai, K.-i.; Takakusa, H.; Kojima, H.; Kikuchi, K.; Ohkubo, K.; Fukuzumi, S.; Nagano, T., Rational Principles for Modulating Fluorescence Properties of Fluorescein. Journal of the American Chemical Society 2004, 126 (43), 14079-14085.

73. He, H.; Mortellaro, M. A.; Leiner, M. J. P.; Young, S. T.; Fraatz, R. J.; Tusa, J. K., A Fluorescent Chemosensor for Sodium Based on Photoinduced Electron Transfer. Analytical Chemistry 2002, 75 (3), 549-555.

74. Gee, K. R.; Zhou, Z.-L.; Qian, W.-J.; Kennedy, R., Detection and Imaging of Zinc Secretion from Pancreatic $\beta$-Cells Using a New Fluorescent Zinc Indicator. Journal of the American Chemical Society 2002, 124 (5), 776-778. 
75. He, H.; Mortellaro, M. A.; Leiner, M. J. P.; Fraatz, R. J.; Tusa, J. K., A Fluorescent Sensor with High Selectivity and Sensitivity for Potassium in Water. Journal of the American Chemical Society 2003, 125 (6), 1468-1469.

76. Komatsu, H.; Iwasawa, N.; Citterio, D.; Suzuki, Y.; Kubota, T.; Tokuno, K.; Kitamura, Y.; Oka, K.; Suzuki, K., Design and Synthesis of Highly Sensitive and Selective Fluorescein-Derived Magnesium Fluorescent Probes and Application to Intracellular 3D Mg2+ Imaging. Journal of the American Chemical Society 2004, $126(50), 16353-16360$.

77. Egawa, T.; Hanaoka, K.; Koide, Y.; Ujita, S.; Takahashi, N.; Ikegaya, Y.; Matsuki, N.; Terai, T.; Ueno, T.; Komatsu, T.; Nagano, T., Development of a Far-Red to Near-Infrared Fluorescence Probe for Calcium Ion and its Application to Multicolor Neuronal Imaging. Journal of the American Chemical Society 2011, 133 (36), 14157-14159.

78. Iyoshi, S.; Taki, M.; Yamamoto, Y., Rosamine-Based Fluorescent Chemosensor for Selective Detection of Silver(I) in an Aqueous Solution. Inorganic Chemistry 2008, 47 (10), 3946-3948.

79. Tang, B.; Cui, L. J.; Xu, K. H.; Tong, L. L.; Yang, G. W.; An, L. G., A Sensitive and Selective Near-Infrared Fluorescent Probe for Mercuric Ions and Its Biological Imaging Applications. ChemBioChem 2008, 9 (7), 1159-1164.

80. Dodani, S. C.; He, Q.; Chang, C. J., A Turn-On Fluorescent Sensor for Detecting Nickel in Living Cells. Journal of the American Chemical Society 2009, 131 (50), 18020-18021.

81. Nolan, E. M.; Lippard, S. J., Small-Molecule Fluorescent Sensors for Investigating Zinc Metalloneurochemistry. Accounts of Chemical Research 2008, 42 (1), 193203.

82. Burdette, S. C.; Frederickson, C. J.; Bu, W.; Lippard, S. J., ZP4, an Improved Neuronal Zn2+ Sensor of the Zinpyr Family. Journal of the American Chemical Society 2003, 125 (7), 1778-1787.

83. Nagano, T., Development of fluorescent probes for bioimaging applications. Proceedings of the Japan Academy, Series B 2010, 86 (8), 837-847. 
84. Sunahara, H.; Urano, Y.; Kojima, H.; Nagano, T., Design and Synthesis of a Library of BODIPY-Based Environmental Polarity Sensors Utilizing Photoinduced Electron-Transfer-Controlled Fluorescence ON/OFF Switching. Journal of the American Chemical Society 2007, 129 (17), 5597-5604.

85. Zhou, X.; Su, F.; Tian, Y.; Youngbull, C.; Johnson, R. H.; Meldrum, D. R., A New Highly Selective Fluorescent K+ Sensor. Journal of the American Chemical Society 2011, 133 (46), 18530-18533.

86. Komatsu, H.; Miki, T.; Citterio, D.; Kubota, T.; Shindo, Y.; Kitamura, Y.; Oka, K.; Suzuki, K., Single Molecular Multianalyte $(\mathrm{Ca} 2+, \mathrm{Mg} 2+)$ Fluorescent Probe and Applications to Bioimaging. Journal of the American Chemical Society 2005, 127 (31), 10798-10799.

87. Xu, Z.; Xiao, Y.; Qian, X.; Cui, J.; Cui, D., Ratiometric and Selective Fluorescent Sensor for CuII Based on Internal Charge Transfer (ICT). Organic Letters 2005, 7 (5), 889-892.

88. Badugu, R.; Lakowicz, J. R.; Geddes, C. D., Enhanced Fluorescence Cyanide Detection at Physiologically Lethal Levels: Reduced ICT-Based Signal Transduction. Journal of the American Chemical Society 2005, 127 (10), 36353641.

89. Srikun, D.; Miller, E. W.; Domaille, D. W.; Chang, C. J., An ICT-Based Approach to Ratiometric Fluorescence Imaging of Hydrogen Peroxide Produced in Living Cells. Journal of the American Chemical Society 2008, 130 (14), 4596-4597.

90. Wang, J.; Qian, X.; Cui, J., Detecting Hg2+ Ions with an ICT Fluorescent Sensor Molecule: Remarkable Emission Spectra Shift and Unique Selectivity. The Journal of Organic Chemistry 2006, 71 (11), 4308-4311.

91. Dujols, V.; Ford, F.; Czarnik, A. W., A Long-Wavelength Fluorescent Chemodosimeter Selective for $\mathrm{Cu}(\mathrm{II})$ Ion in Water. Journal of the American Chemical Society 1997, 119 (31), 7386-7387.

92. Chen, X.; Ma, H., A selective fluorescence-on reaction of spiro form fluorescein hydrazide with $\mathrm{Cu}(\mathrm{II})$. Analytica Chimica Acta 2006, 575 (2), 217-222. 
93. Jung Jou, M.; Chen, X.; Swamy, K. M. K.; Na Kim, H.; Kim, H.-J.; Lee, S.-g.; Yoon, J., Highly selective fluorescent probe for Au3+ based on cyclization of propargylamide. Chemical Communications 2009, (46), 7218-7220.

94. Tang, B.; Ding, B.; Xu, K.; Tong, L., Use of Selenium to Detect Mercury in Water and Cells: An Enhancement of the Sensitivity and Specificity of a Seleno Fluorescent Probe. Chemistry - A European Journal 2009, 15 (13), 3147-3151.

95. Wu, D.; Huang, W.; Duan, C.; Lin, Z.; Meng, Q., Highly Sensitive Fluorescent Probe for Selective Detection of $\mathrm{Hg} 2+$ in DMF Aqueous Media. Inorganic Chemistry 2007, 46 (5), 1538-1540.

96. Koide, Y.; Urano, Y.; Hanaoka, K.; Terai, T.; Nagano, T., Development of an SiRhodamine-Based Far-Red to Near-Infrared Fluorescence Probe Selective for Hypochlorous Acid and Its Applications for Biological Imaging. Journal of the American Chemical Society 2011, 133 (15), 5680-5682.

97. Lippert, A. R.; Van de Bittner, G. C.; Chang, C. J., Boronate Oxidation as a Bioorthogonal Reaction Approach for Studying the Chemistry of Hydrogen Peroxide in Living Systems. Accounts of Chemical Research 2011, 44 (9), 793-804.

98. Tang, B.; Yin, L.; Wang, X.; Chen, Z.; Tong, L.; Xu, K., A fast-response, highly sensitive and specific organoselenium fluorescent probe for thiols and its application in bioimaging. Chemical Communications 2009, (35), 5293-5295.

99. Zhang, W.; Tang, B.; Liu, X.; Liu, Y.; Xu, K.; Ma, J.; Tong, L.; Yang, G., A highly sensitive acidic $\mathrm{pH}$ fluorescent probe and its application to HepG2 cells. Analyst 2009, 134 (2), 367-371.

100. Kobayashi, H.; Ogawa, M.; Alford, R.; Choyke, P. L.; Urano, Y., New Strategies for Fluorescent Probe Design in Medical Diagnostic Imaging. Chem Rev 2009, 110 (5), 2620-2640. 


\section{Chapter 2 : Highly Water-soluble BODIPY-based Fluorescent Probe for Sensitive and Selective Detection of Nitric Oxide in Living Cells ${ }^{1}$}

Giri Vegesna, ${ }^{a}$ Srinivas R. Sripathi, ${ }^{b}$ Jingtuo Zhang, ${ }^{a}$ Shilei Zhu, ${ }^{a}$ Weilue He, ${ }^{c}$ Fen-Tair Luo, ${ }^{\mathrm{d}}$ Wan Jin Jahng, ${ }^{\mathrm{b}}$ Megan Frost, ${ }^{\mathrm{c}}$ and Haiying Liu ${ }^{\mathrm{a} *}$

a Department of Chemistry, ${ }^{b}$ Department of Biological Science, ${ }^{c}$ Department of Biomedical Engineering, Michigan Technological University, Houghton, MI 49931

${ }^{\mathrm{d}}$ Institute of Chemistry, Academia Sinica, Taipei, Taiwan 11529, Republic of China

\footnotetext{
${ }^{1}$ The material included in this chapter was previously published in the ACS Appl. Mater. Interfaces, 2013, 5 (10), pp 4107-4112 DOI: 10.1021/am303247s Publication Date (Web): April 24, 2013Copyright (C) 2013 American Chemical Society http://pubs.acs.org/doi/abs/10.1021/am303247
} 


\subsection{Abstract}
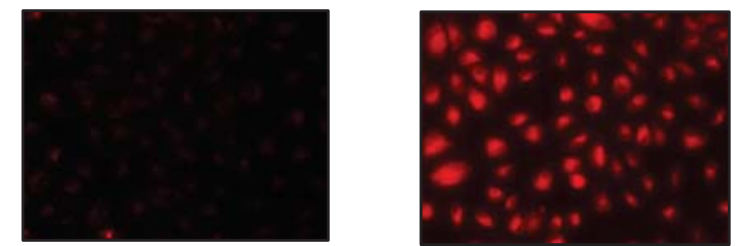

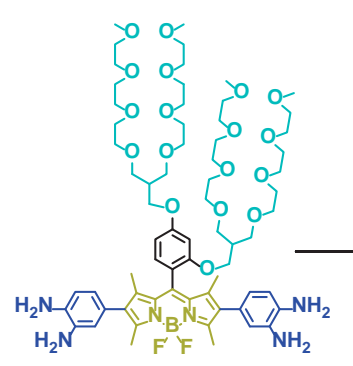

A

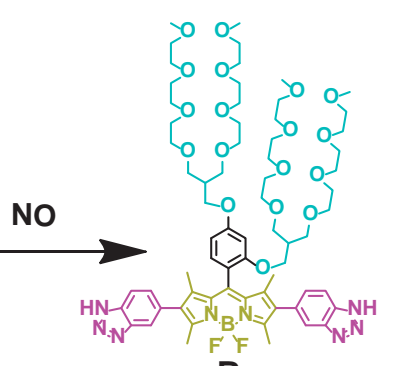

B

Figure 2.1 Fluorescent probes A and B and their cell images

A highly water-soluble BODIPY dye bearing electron-rich o-diaminophenyl groups at 2,6positions was prepared as a highly sensitive and selective fluorescent probe for detection of nitric oxide (NO) in living cells. The fluorescent probe displays an extremely weak fluorescence with fluorescence quantum yield of 0.001 in $10 \mathrm{mM}$ phosphate buffer $(\mathrm{pH}$ 7.0) in the absence of NO as two electron-rich o-diaminophenyl groups at 2,6-positions significantly quench the fluorescence of the BODIPY dye via photoinduced electron transfer mechanism. The presence of $\mathrm{NO}$ in cells enhances the dye fluorescence dramatically. The fluorescent probe demonstrates excellent water solubility, membrane permeability and compatibility with living cells for sensitive detection of NO.

Keywords: BODIPY dyes, Fluorescent probe, Nitric Oxide, Cell imaging 


\subsection{Introduction}

Nitric oxide (NO) is a highly reactive free-radical gas as a ubiquitous signaling molecule that is responsible for both physiologically positive as well as pathogenic states in many biological systems. ${ }^{1-7}$ NO has been shown to have anti-apoptotic effects in endothelial cells, lymphoma cells, ovarian follicles, cardiac myocytes and hepatocytes. ${ }^{2}$ In apparent contradiction to this, $\mathrm{NO}$ has been shown to possess pro-apoptotic properties in macrophages, neurons, pancreatic $\beta$-cells, thymocytes chondrocytes and hepatocytes. ${ }^{3} \mathrm{NO}$ has shown to have beneficial antiviral, antimicrobial, antiparisital, immunomodulary, and antitumoral effects when induced at the right time and place. Reviews suggest $\mathrm{NO}$ in low levels has a protective and proliferative effect on some cells while at high levels induces cell cycle arrest, apoptosis and senescence. ${ }^{1,6-7}$ The pathways that regulate nitric oxide synthase (NOS), the enzyme responsible for endogenous production of NO, differ between cells and species. ${ }^{8}$ The regulation of NO production can occur at the transcription, posttranscriptional, translational, and posttranslational level. The observed effects of NO are dependent on a number of factors including flux of NO, the timing of NO release, the accumulated dose of NO, and the type of cells exposed to the NO. Clearly defining how much NO is needed and for what duration it is produced to induce the array of activity it has on biological systems is essential to enabling better understanding of the role NO plays in various pathways.

Accurate detection and quantification of $\mathrm{NO}$ will provide effective approaches for better understanding of its origins, activities and biological functions. A variety of methods such as electron paramagnetic resonance spectroscopy, ${ }^{9}$ colorimetric, ${ }^{10}$ fluorometric, ${ }^{11-29}$ electrochemical, ${ }^{30-31}$ and chemiluminescence techniques ${ }^{31}$ have been developed to detect and monitor endogenous $\mathrm{NO}$ for insightful understanding of the complicated functions of NO in living systems. Among these methods, metal-based and o-diaminophenyl-based fluorescent probes have been shown very promising to visualize NO in vitro and in vivo at cellular level under physiological conditions because of its high sensitivity, selectivity, real-time and simplicity. ${ }^{11-26}$ The response mechanism is based on irreversible reaction of o-diaminophenyl group of the fluorescent probe with by-products such as the autooxidation 
of NO to turn on the probe fluorescence via formation of a strongly fluorescent benzotriazole derivative. ${ }^{11-25}$ However, some of these fluorescent probes show different undesirable properties such as poor compatibility with living cells, low water solubility or membrane permeability.

In this paper, we chose BODIPY (4,4'-difluoro-4-bora-3a,4a-diaza-s-indacene) dye as a fluorophore because of its many distinctive and desirable properties such as high absorption coefficients, narrow absorption bands, sharp emissions, high fluorescence quantum yields, and excellent chemical and photostabilities. ${ }^{32-35}$ We report highly watersoluble BODIPY-based dye bearing electron-rich o-diaminophenyl groups at 2,6-positions as a sensitive and selective fluorescent probe for $\mathrm{NO}$ by introducing branched oligo(ethylene glycol)methyl ether residues to BODIPY dye at the meso-position, and two electron-rich o-diaminophenyl groups at 2,6-positions as fluorescent sensing switches. The fluorescent probe was prepared by palladium-catalyzed Suzuki coupling reaction of (4amino-3-nitrophenyl)boronic acid with 2,6-diiodo-BODIPY dye in a basic condition and followed by reduction of nitro moieties of 3-amino-4-nitrophenyl groups to amine groups. The fluorescent probe displays sensitive response to $\mathrm{NO}$ as the presence of $\mathrm{NO}$ significantly enhances the fluorescence intensity of the fluorescence probe by reducing effectiveness of photoinduced electron transfer of electron-rich o-diaminophenyl groups at 2,6-positions via irreversible oxidation of the o-diaminophenyl groups. The fluorescent probe possesses excellent water solubility, membrane permeability and compatibility with living cells for sensitive detection of NO in living cells.

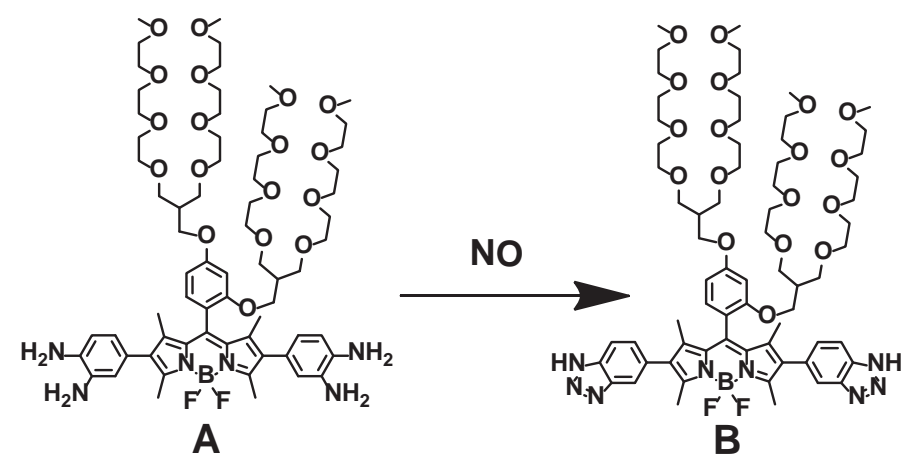

Scheme 2.1. BODIPY-based fluorescent probe for NO. 


\subsection{Experimental section}

\subsubsection{Materials}

Fluorescent probe A: ${ }^{1} \mathrm{H}$ NMR $\left(400 \mathrm{MHz}, \mathrm{CDCl}_{3}\right): \delta 7.00(\mathrm{~d}, \mathrm{~J}=8.4 \mathrm{~Hz}, 1 \mathrm{H}), 6.66(\mathrm{~d}, \mathrm{~J}$ $=8 \mathrm{~Hz}, 2 \mathrm{H}), 6.56(\mathrm{dd}, \mathrm{J}=8.4,2.4 \mathrm{~Hz}, 1 \mathrm{H}), 6.51(\mathrm{~d}, \mathrm{~J}=2 \mathrm{~Hz}, 1 \mathrm{H}), 6.48(\mathrm{~d}, \mathrm{~J}=2 \mathrm{~Hz}, 2 \mathrm{H})$, $6.45(\mathrm{dd}, \mathrm{J}=8,1.6 \mathrm{~Hz}, 2 \mathrm{H}), 4.02(\mathrm{~d}, \mathrm{~J}=6 \mathrm{~Hz}, 2 \mathrm{H}), 3.95$ (d, J = 5.6 Hz, 2H), 3.61-3.43 (m, $50 \mathrm{H}), 3.38-3.24(\mathrm{~m}, 18 \mathrm{H}) 2.48(\mathrm{~s}, 6 \mathrm{H}), 2.42-2.34(\mathrm{~m}, 1 \mathrm{H}), 2.32-2.22(\mathrm{~m}, 1 \mathrm{H}), 1.39(\mathrm{~s}, 6 \mathrm{H})$. ${ }^{13} \mathrm{C}$ NMR (100 MHz, $\left.\mathrm{CDCl}_{3}\right): \delta 161.49,156.80,153.57,139.06,138.37,134.81,133.84$, $133.54,131.96,129.98,125.74,122.17,118.58,117.21,116.59,106.25,100.38,72.12$, $70.83,70.82,70.80,70.77,70.73,70.68,70.66,70.50,69.57,69.30,66.60,66.27,59.21$, 59.17, 40.20, 39.74, 13.58, 12.59. IR ( $\left.\mathrm{cm}^{-1}\right): 3355,2870,1609,1533,1463,1388,1287$, 1203, 1177, 1096, 1004, 849, 767, 707. HRMS (FAB) calcd for $\mathrm{C}_{67} \mathrm{H}_{103} \mathrm{~N}_{6} \mathrm{~F}_{2} \mathrm{BO}_{18}[\mathrm{M}]^{+}$, 1328.7390; found, 1328.7407.

\subsubsection{NOC-7 treatment and fluorescence imaging}

Murine macrophages (RAW 264.7) or human retinal pigment epithelial cells (ARPE-19) were washed twice with phosphate buffered saline (PBS) to remove the traces of serum and incubated with the final concentration of $5 \mu \mathrm{M}$ fluorescent probe A in FBS (fetal bovine serum)-free DMEM (Dulbecco's modified Eagle medium) for $4 \mathrm{~h}$ at $37^{\circ} \mathrm{C}$. After being repeatedly washed with PBS, cells were treated with $4 \mu \mathrm{M}$ of nitric oxide (NO) donor NOC-7 (Santa Cruz Biotechnology Inc, CA) in serum free DMEM for 1-2 h. Untreated cells with NOC-7 are considered as control. Differential interference contrast (DIC) and fluorescence images were acquired using a Zeiss AxioVert 200 M Apo Tome fluorescent microscope with 20X magnification, with an exposure time of $500 \mathrm{~ms}$ for murine macrophages and one second for human retinal pigment epithelial cells. Emission from the fluorescent probe A was detected with the red filter set (with excitation at $540 \mathrm{~nm}$ ). 


\subsubsection{LPS treatment and fluorescence imaging}

For studies of endogenously generated NO detection, lipopolysaccharide (LPS) was used to stimulate NO through iNOS (inducible nitric oxide synthase) for $16 \mathrm{~h}$. After murine macrophage cells were washed with PBS for 3 times, $100 \mathrm{ng} / \mathrm{mL}$ LPS in FBS-free media was applied to cells. After 16-h incubation with LPS, the cells were washed by PBS for 3 times and $10 \mu \mathrm{M}$ fluorescent probe A dissolved in FBS-free media was applied to cells. 10 $\mu \mathrm{M}$ 4, 5-diaminofluorescein diacetate (DFA) was used as a control for comparison purpose. After one-hour incubation, cells were washed by PBS for 3 times and living cell was mounted onto Zeiss AxioVert $200 \mathrm{M}$ inverted microscope for imaging. Images were obtained at excitation of $500 \mathrm{~nm}$.

\subsection{Results and Discussion}

\subsubsection{Synthetic approach to fluorescent probe for NO}

In order to prepare highly water-soluble BODIPY-based fluorescent probe for NO, we introduced branched oligo(ethylene glycol)methyl ether residues to BODIPY dyes at the meso positions and electron-rich o-diaminophenyl groups at 2,6-positions as fluorescent switches to BODIPY dye (Scheme 2.2). We demonstrated effective use of branched oligo(ethylene glycol)methyl ether residues to achieve high water solubility of BODIPY dyes with emissions ranging from green to near-infrared regions by introducing these residues to BODIPY dyes at all positions such as the meso, 2,6-positions, 3-position, 3,5positions, 1,7-positions, 1,3,5,7-positions or 4,4'-positions. ${ }^{36-37}$ In order to control effective turn-on switch for sensitive detection of NO, we used an ortho-substituent group of branched oligo(ethylene glycol)methyl ether on the meso-phenyl ring of BODIPY dye as this approach has been reported to effectively enhance fluorescence quantum yield of BODIPY dye in aqueous solution by preventing dye aggregation resulting from $\pi-\pi$ stacking interactions among BODIPY dyes and free rotation of the meso-phenyl ring of BODIPY dye. ${ }^{37}$ BODIPY dye bearing branched oligo(ethylene glycol)methyl ether at the meso position (4) was prepared according to our reported procedure, starting from 2,4- 
dihydroxybenzaldehyde (1) (Scheme 2).${ }^{37}$ 2,6-Diiodo-BODIPY dye (5) was prepared by iodonization of BODIPY dye 4 at 2,6-positions in the presence of iodine and iodic acid in mixed solution of ethanol and water. ${ }^{37-39}$ BODIPY dye bearing 4-amino-3-nitrophenyl groups at 2,6-positions (8) was prepared by palladium-catalyzed Suzuki coupling reaction of (4-amino-3-nitrophenyl)boronic acid (7) with 2,6-diiodo-BODIPY dye (5) in a basic condition under a nitrogen atmosphere (Scheme 2.2). ${ }^{39}$ BODIPY dye bearing electron-rich o-diaminophenyl groups at 2,6-positions (fluorescent probe A) was prepared by reduction of nitro moieties from 4-amino-3-nitrophenyl groups at 2,6-positions of BODIPY dye (8) to amine groups in the presence of hydrazine hydrate and palladium on carbon in ethanol solution.

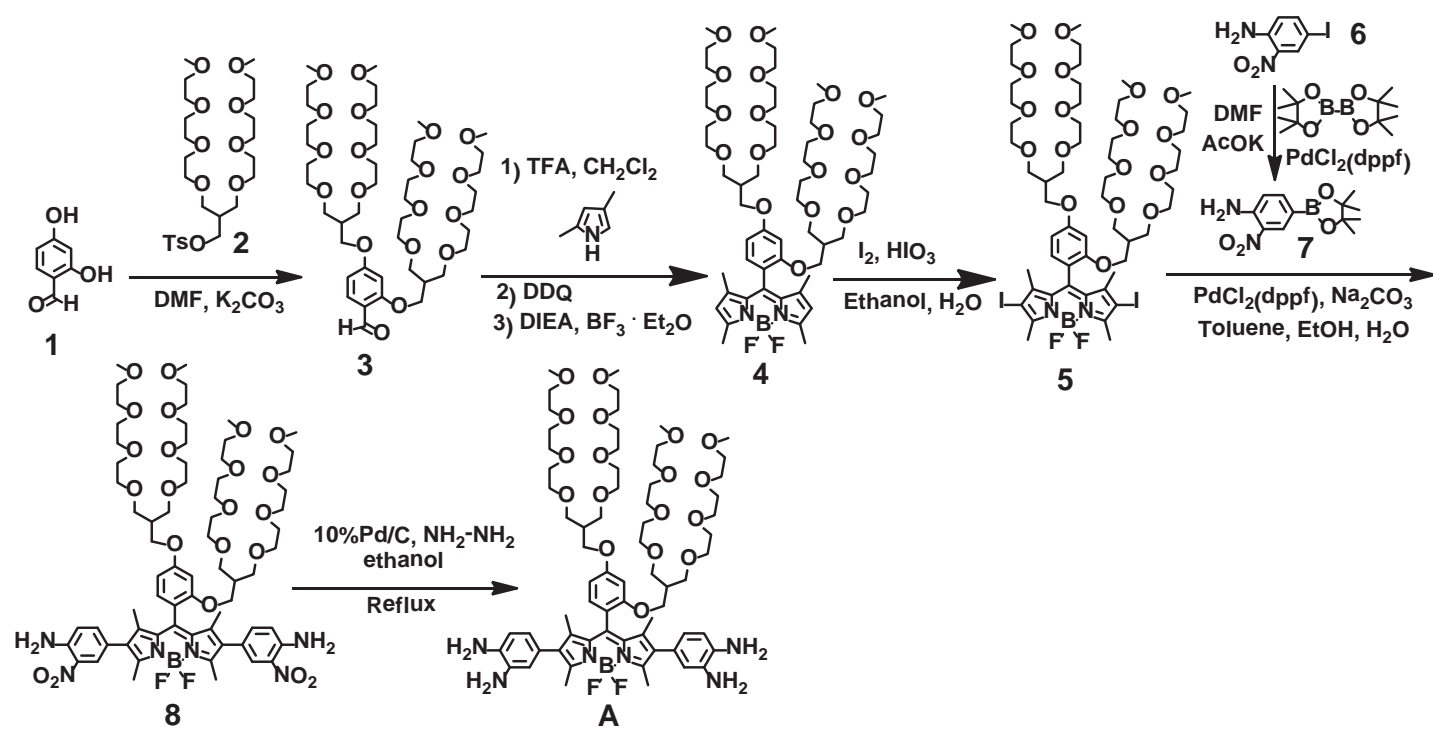

Scheme 2.2 Synthetic route to BODIPY-based fluorescent probe A for NO.

\subsubsection{Optical properties of BODIPY dyes}

The absorbance and fluorescence properties, and fluorescence lifetimes of BODIPY dyes 4, 5, 8 and fluorescent probe A are summarized in Table 2.1. All BODIPY dyes 4, 5 and A except 8 are highly soluble not only in water but also in common organic solvents such as tetrahydrofuran, dichloromethane, chloroform, DMSO, DMF and ethyl acetate. 
BODIPY dye 4 in PBS solution shows a strong absorption peak at $501 \mathrm{~nm}$ due to $\mathrm{S}_{0} \rightarrow \mathrm{S}_{1}$ $\left(\pi-\pi^{*}\right)$ transition and a weaker broad band around $350 \mathrm{~nm}$ attributed to the $\mathrm{S}_{0} \rightarrow \mathrm{S}_{2}\left(\pi-\pi^{*}\right)$ transition $^{32,34-35}$ and displays fluorescence peak at $511 \mathrm{~nm}$ with high fluorescence quantum yield of 0.32 (Table 2.1). The high fluorescence quantum yield is attributed to enhanced steric hindrance of the bulky ortho-substituent group on the meso-phenyl ring of BODIPY dye 4 , that can effectively prevent free rotation of the meso-phenyl ring and potential $\pi-\pi$ stacking interactions between the BODIPY cores in aqueous solution. ${ }^{37}$ 2,6-DiiodoBODIPY dye (5) displays large red shifts ( $34 \mathrm{~nm}$ and $42 \mathrm{~nm}$ ) in both the absorption and fluorescence maxima, respectively compared with BODIPY dye 4 as 2,6-diiodo substituents serve as auxochromes. ${ }^{39}$ However, it exhibits low fluorescence quantum yield of 0.009 with short fluorescence lifetime of $0.026 \mathrm{~ns}$ in PBS solution because of efficient intersystem crossing induced by the heavy atom effect of iodine. ${ }^{4 a, 4 b}$ BODIPY dye bearing 4-amino-3-nitrophenyl groups at 2,6-positions (8) displays poor water solubility in aqueous solution because a hydrophobic feature of 4-amino-3-nitrophenyl groups at 2,6-positions makes the BODIPY core more hydrophobic. It shows absorption and emission peaks at $534 \mathrm{~nm}$ and $592 \mathrm{~nm}$ with fluorescence quantum yield of 0.084 in acetonitrile solution. Fluorescent probe A displays absorption and emission peaks at $539 \mathrm{~nm}$ and $570 \mathrm{~nm}$, respectively, and shows very weak fluorescence with fluorescence quantum yield of 0.001 in phosphate buffer $(\mathrm{pH}$ 7.0) because two electron-rich o-diaminophenyl groups at 2,6positions significantly quench the fluorescence of the BODIPY dye via photoinduced electron transfer mechanism. ${ }^{11-24}$ But it can become highly fluorescent after it reacts with NO to generate the electron-deficient product with the triazole rings, which cannot engender photoinduced electron transfer. ${ }^{11-24}$ 


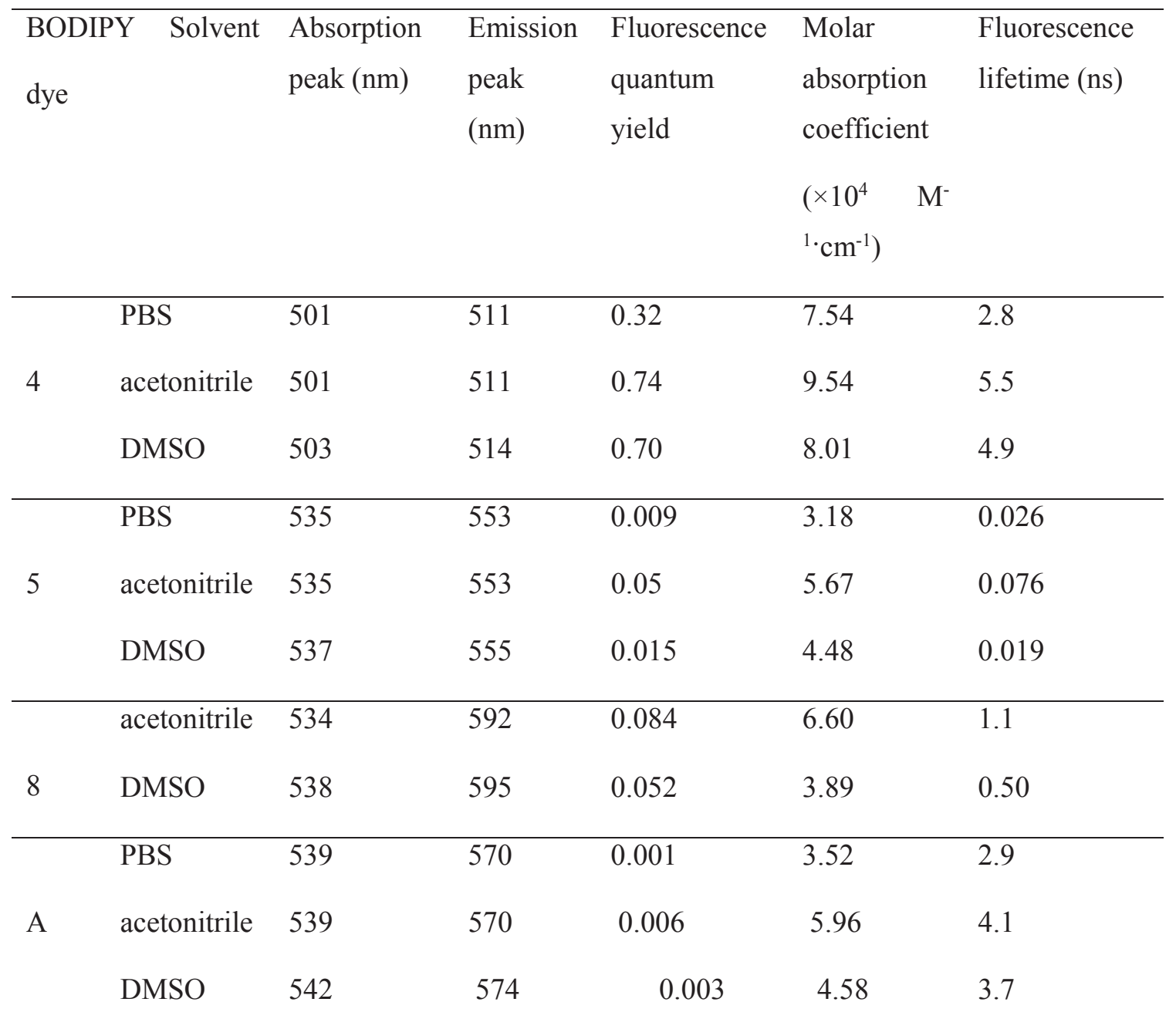

Table 2.1. Optical properties, fluorescence lifetimes and fluorescence quantum yields of BODIPY dyes 4, 5 and A in $10 \mathrm{mM}$ phosphate buffer solution (PBS) (pH 7.0), acetonitrile and DMSO, and BODIPY dye 8 in acetonitrile and DMSO.

\subsubsection{The fluorescent response and selectivity of the fluorescent probe A to NO}

We examined the performance of BODIPY-based fluorescent probe to detect NO in aqueous solution by conducting a titration experiment with a NO-saturated stock solution 
and a fluorescent probe solution $(5 \mu \mathrm{M})$ in $10 \mathrm{mM}$ phosphate buffer at $\mathrm{pH}$ 7.0. The fluorescence intensity of the fluorescent probe is significantly enhanced upon addition of the NO solution. In addition, the concentration dependent fluorescence enhancement displays a linearity from 1.7 to $8.3 \mu \mathrm{M}$ with the detection limit $(\mathrm{S} / \mathrm{N}=3)$ of $5.0 \times 10^{-7} \mathrm{M}$ (Figure 2.2). The detection limit of 4,5-diaminofluorescein diacetate for NO is $5 \mathrm{~nm} .{ }^{40}$ The detection limit of fluoresceinamine for $\mathrm{NO}$ is $44 \mathrm{~nm}^{41}$ A detection sensitivity limit of 6.3 $\mathrm{nM}$ in a mixed solution of dichloromethne and ethanol $(4: 1, \mathrm{v} / \mathrm{v})$ was reported in NO sensor of metal-based conjugated polymer. ${ }^{42}$ The increase of the probe fluorescence intensity arises from formation of a strongly fluorescent benzotriazole derivative through reaction of $o$-diaminophenyl groups of the fluorescent probe at 2,6-positions with NO-derivated byproducts such as the autooxidation of NO, which prevents fluorescence quenching via photoinduced electron transfer from $o$-diaminophenyl groups. ${ }^{11-24}$ The reaction product, BODIPY dye B (Scheme 2.1), was determined by electrospray mass spectrometry and an ion peak was found at $1349.7(\mathrm{~m} / \mathrm{z})(\mathrm{M}-\mathrm{H})^{+}$, corresponding to the ion that one proton was removed from BODIPY dye B.

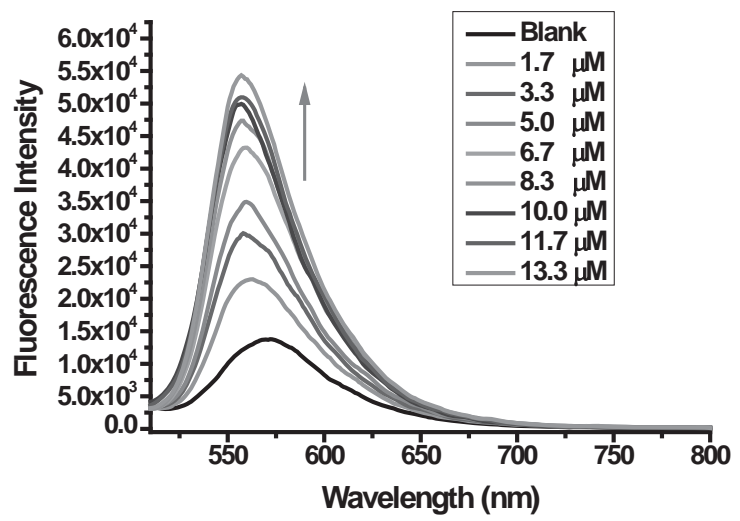

Figure 2.2. Fluorescence spectra of $5 \mu \mathrm{M}$ fluorescent probe $\mathrm{A}$ in the absence and presence of different amounts of NO stock solution in a phosphate buffer $(10 \mathrm{mM}, \mathrm{pH} 7.0)$ at room temperature. 


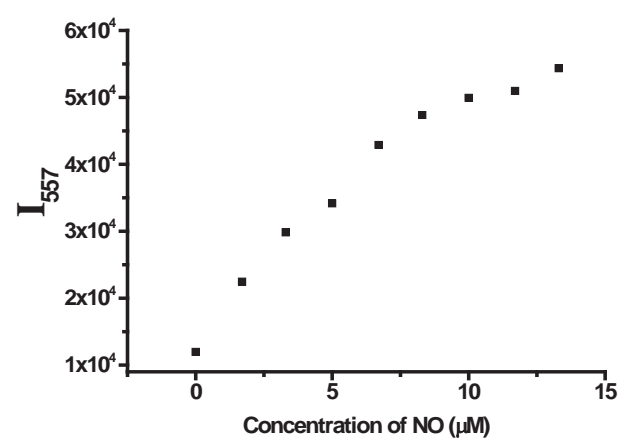

Figure 2.3 Fluorescent intensity change at $557 \mathrm{~nm}\left(\mathrm{I}_{557}\right)$ of $5 \mu \mathrm{M}$ fluorescent probe upon titration of NO stock solution $(0-13.3 \mu \mathrm{M})$

The selective experiments were conducted by addition of other reactive nitrogen species (RNS) and oxygen species (ROS) to the fluorescent probe in $10 \mathrm{mM}$ phosphate buffer ( $\mathrm{pH}$ 7.0) (Figures 2.4 and 2.5). The fluorescent probe shows high specificity for NO over other reactive species present in the biological milieu. Except $\mathrm{OCl}^{-}$, other RNS and ROS such as $\mathrm{NO}_{2}, \mathrm{O}_{2}{ }^{-}, \mathrm{NO}_{3}{ }^{-}, \mathrm{H}_{2} \mathrm{O}_{2}$, and $\mathrm{OH}$ failed to induce significant emission enhancement of the fluorescent probe (Figure 2.4), that makes this dye a unique tool to measure levels of NO in biological samples and help understand their role in health and disease. This probe showed similar selectivity as 4,5-diaminofluorescein diacetate. ${ }^{40}$

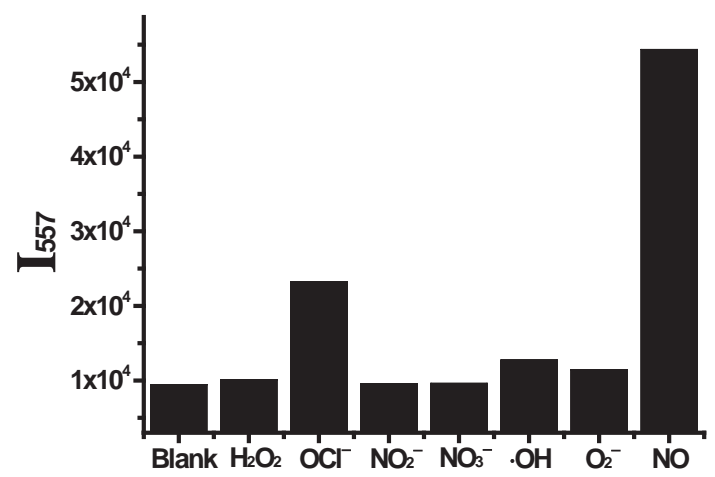

Figure 2.4. The fluorescent responses of $5 \mu \mathrm{M}$ fluorescent probe A to different ROS and RNS $(100 \mu \mathrm{M}$, NO: $13.3 \mu \mathrm{M})$ in $10 \mathrm{mM}$ phosphate buffer (pH 7.0) at $557 \mathrm{~nm}$. 


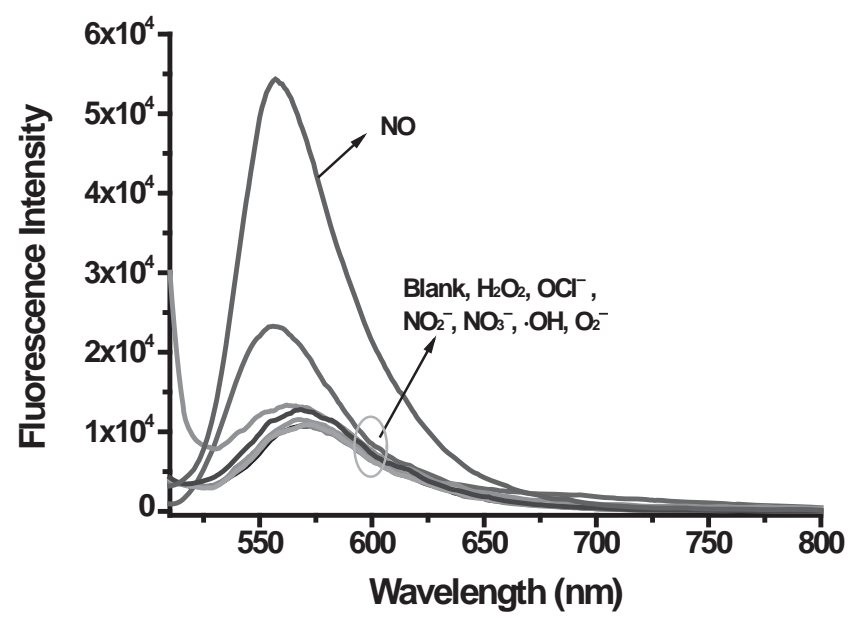

Figure 2.5. The fluorescent spectra of $5 \mu \mathrm{M}$ fluorescent probe $\mathrm{A}$ upon addition of different ROS and RNS (100 $\mu \mathrm{M}, \mathrm{NO}: 13.3 \mu \mathrm{M})$ in phosphate buffer (10 mM, pH 7.0).

\subsubsection{Use of fluorescent probe to detect nitric oxide in living cells}

NO is a highly reactive free-radical gas that is involved in protein nitration. Studies suggest that NO plays a specific protective and cytotoxic role in cells. Clearly defining how much NO is needed and for what duration it is produced to induce protein nitration is critical because excess levels of $\mathrm{NO}$ are toxic and will lead to tissue damage and cell death. The fluorescence imaging technique is a very important tool for in vivo and in situ monitoring of NO.

To evaluate the feasibility of using fluorescent probe A to detect NO in cells, we tested fluorescent probe A in human-derived retinal pigment epithelium (ARPE-19) and murine macrophages (RAW 264.7). First cells were incubated with $5 \mu \mathrm{M}$ fluorescent probe A in FBS-free DMEM at $37^{\circ} \mathrm{C}$ for $4 \mathrm{~h}$, and then washed three times with PBS buffer. After that $4 \mu \mathrm{M}$ NOC-7 (1-hydroxy-2-oxo-3-(N-3-methyl-aminopropyl)-3-methyl-1-triazene), an exogeneous $\mathrm{NO}$ donor with a half-life time of $5 \mathrm{~min}$ at a concentration of $0.1 \mathrm{mM}$ in $0.1 \mathrm{M}$ PBS (pH 7.4), ${ }^{43}$ was added and incubated for 1 or $2 \mathrm{~h}$, and cells were washed thrice with PBS. We observed very weak fluorescence of the cells in the absence of NOC-7 (Figures 2.6C, 2.6D, 2.7C and 2.7D). The purpose of adding the NO donor was to ensure modulation of the NO levels in the cells and that the NO was detected by the probe. This was indeed 
the case, with an increase in the addition of NOC-7 an increase in fluorescence was observed for both cell types (Figures $2.6 \mathrm{G}$ and $2.7 \mathrm{G}$ ). In addition, the fluorescence intensity in both cells increased significantly by longer time ( $2 \mathrm{~h}$, Figures $2.6 \mathrm{H}$ and $2.7 \mathrm{H})$. These results confirm that the fluorescent probe A is turned on by $\mathrm{NO}$ and its fluorescent intensity depends upon the levels of $\mathrm{NO}$ in solution. In addition, the fluorescent probe A is firmly trapped within a cell without an issue of diffusing out of cells after its initial loading, which was reported in metal-based florescent NO probe. ${ }^{18}$

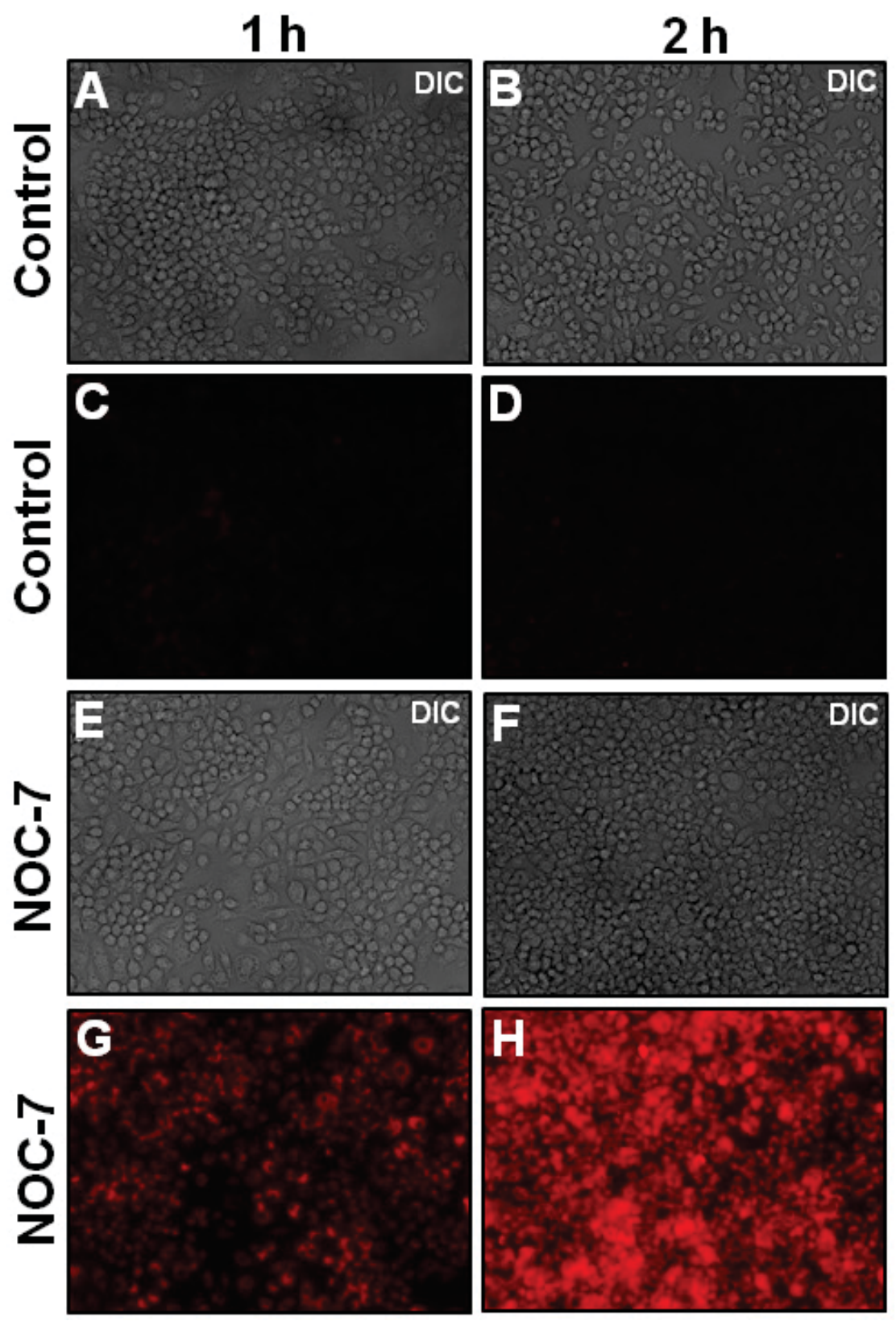


Figure 2.6. Visualization of NO produced in macrophages (RAW 264.7) using $5 \mu \mathrm{M}$ fluorescent probe A after supply with NOC-7 (4 $\mu \mathrm{M})$ for one or two h. (A) Bright-field, $1 \mathrm{~h}$, (B) Bright-field, 2h, (C) Fluorescence, 1h, (D) Fluorescence, 2h, (E) Bright-field, NOC-7, 1h, (F) Bright-field, NOC-7, 2 h, (G) Fluorescence, NOC-7,1 h, (H) Fluorescence, NOC$7,2 \mathrm{~h}$.

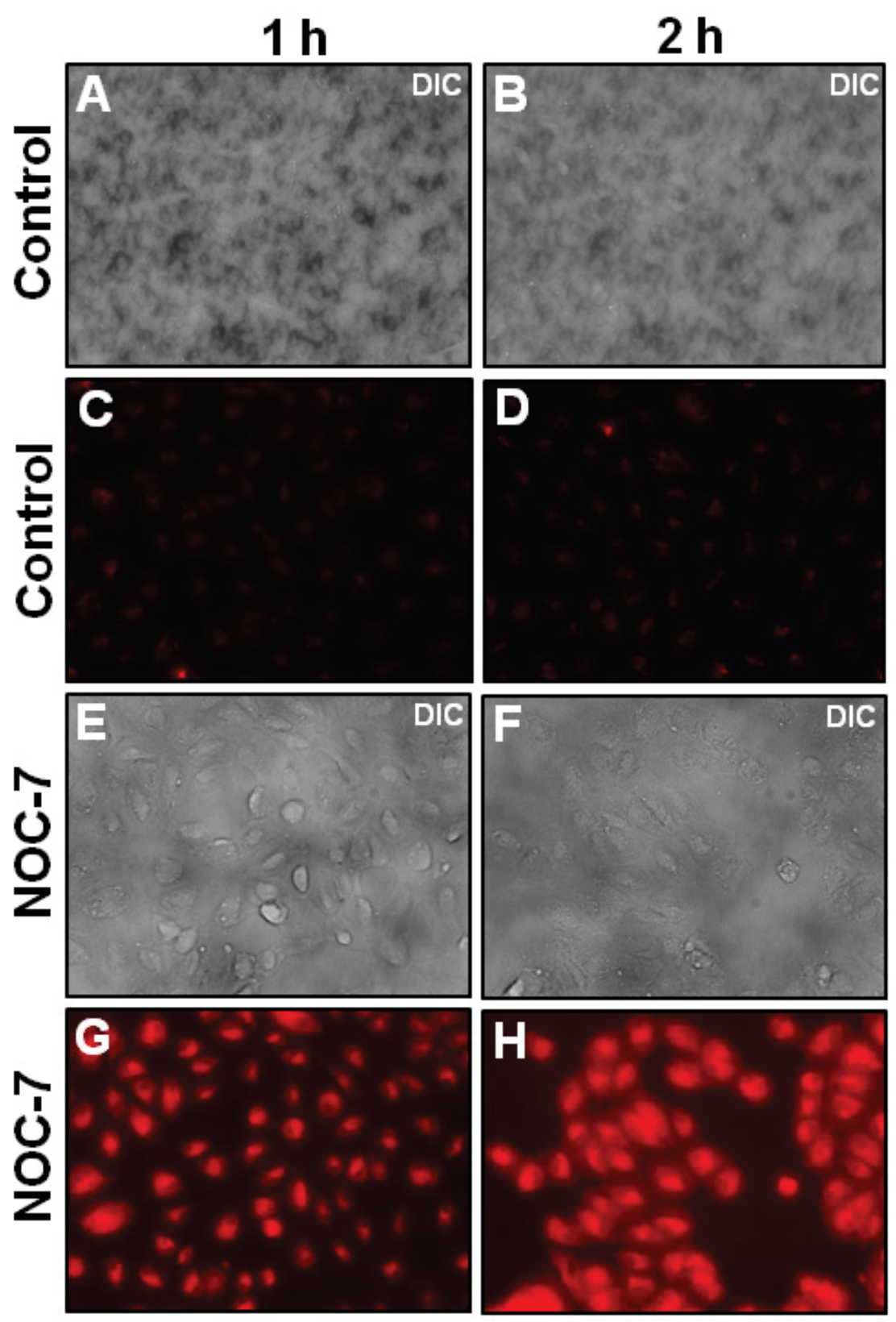


Figure 2.7. Visualization of NO produced in retinal pigment epithelial cells (ARPE-19) using $5 \mu \mathrm{M}$ fluorescent probe A after supply with NOC-7 $(4 \mu \mathrm{M})$ for one or two h. (A) Bright-field,1h, (B) Bright-field, 2h, (C) Fluorescence, 1h, (D) Fluorescence, 2h, (E) Bright-field, NOC-7, 1h, (F) Bright-field, NOC-7, 2 h, (G) Fluorescence, NOC-7,1 h, (H) Fluorescence, NOC-7, 2 h.

We performed fluorescence microscopic imaging of biologically produced NO in RAW 264.7 murine macrophages in order to evaluate the utility of fluorescent probe A to detect endogenously generated NO (Figure 2.8). The RAW 264.7 murine macrophages were stimulated by lipopolysaccharide (LPS; $100 \mathrm{ng} / \mathrm{mL}$ ) for $16 \mathrm{~h}$ and followed by further incubation with $10 \mu \mathrm{M}$ fluorescent probe A for one hour. In a control experiment, cells were incubated for $16 \mathrm{~h}$ with fluorescent probe $\mathrm{A}$ in the absence of LPS. In addition, we use the commercially available NO fluorescent probe, 4,5-diaminofluorescein diacetate (DAF-2 DA) as a control for the purpose of comparison to probe A. A noticeable fluorescence increase in RAW 264.7 murine macrophages was observed at $1 \mathrm{~h}$ incubation with fluorescent probe A after induction of nitric oxide synthase while it was not observed in the control cells, indicating that fluorescent probe A can detect endogenously produced NO. The similar result was observed in control experiments by using DAF-2 DA (Figure A.15 in supporting information). The sensitivity of the commercial fluorescent probe (DAF-2 DA) for NO is a little higher than that of this BODIPY-based fluorescent probe in cell imaging. However, the commercially fluorescent probe shows higher fluorescent background in the cells without LPS stimulation under the identical condition (Figure A.15 in supporting information). 

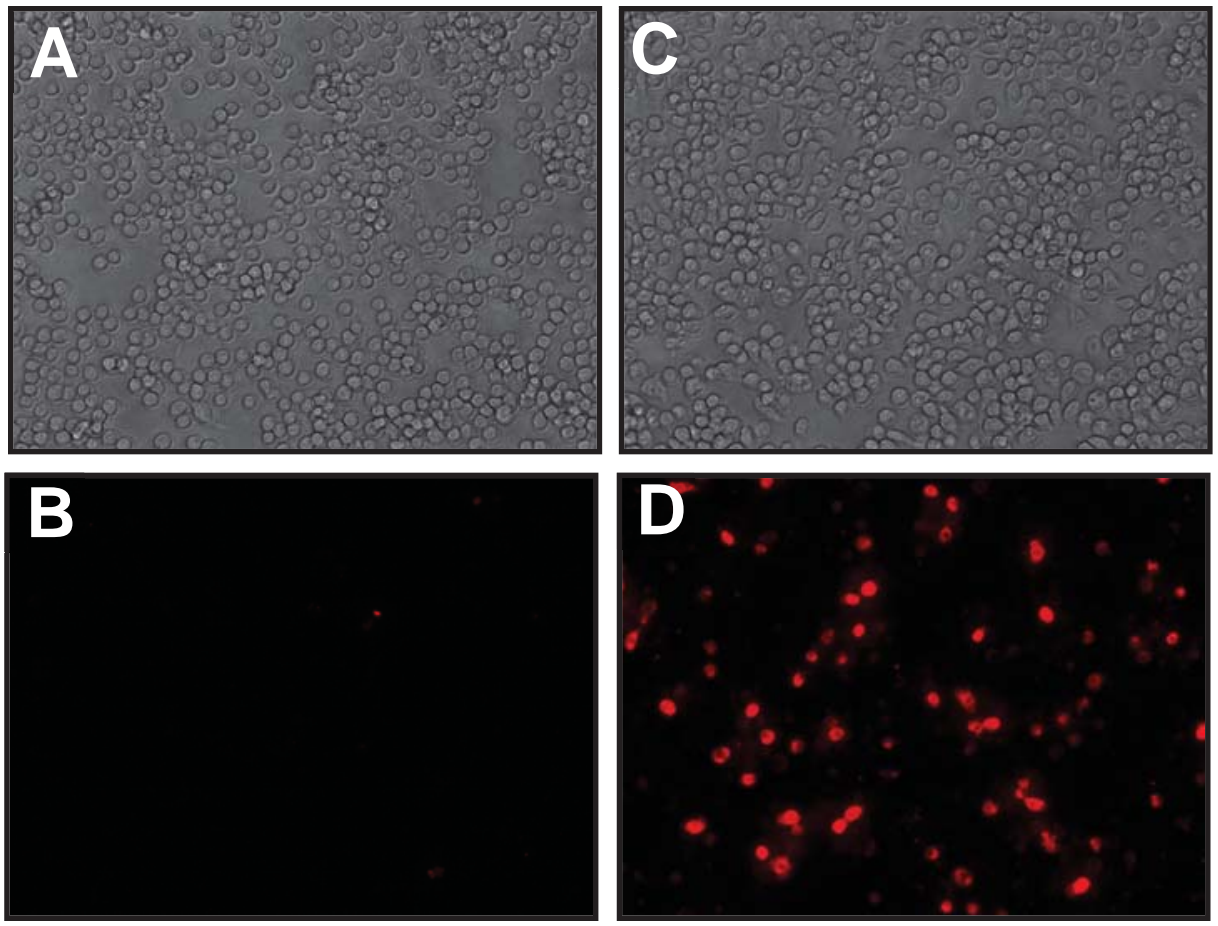

Figure 2.8. Endogenous NO detection in RAW 264.7 murine macrophages by $10 \mu \mathrm{M}$ fluorescent probe A without and with stimulation of $100 \mathrm{ng} / \mathrm{mL}$ lipopolysaccharide. (A) Bright-field,1h, (B) Fluorescence, 1h (C) Bright-field, LPS stimulation, 1h, (D) Fluorescence, LPS stimulation, $1 \mathrm{~h}$.

\subsection{Conclusion}

We have prepared a highly water-soluble BODIPY-based fluorescent probe for sensitive and selective detection of NO. The fluorescent probe shows differential response to the levels of NO produced endogenously in live murine macrophages and exogenously in human retinal pigment epithelial cells.

Author information

Corresponding author

E-mail: hyliu@,mtu.edu 
Notes

The authors declare no competing financial interest.

\section{ACKNOWLEDGMENTS}

This work was partially supported by National Science Foundation (to H.Y. Liu).

\section{References}

1. Thomas, D. D.; Ridnour, L. A.; Isenberg, J. S.; Flores-Santana, W.; Switzer, C. H.; Donzelli, S.; Hussain, P.; Vecoli, C.; Paolocci, N.; Ambs, S.; Colton, C. A.; Harris, C. C.; Roberts, D. D.; Wink, D. A., The chemical biology of nitric oxide: Implications in cellular signaling. Free Radic. Biol. Med. 2008, 45 (1), 18-31.

2. Blaise, G. A.; Gauvin, D.; Gangal, M.; Authier, S., Nitric oxide, cell signaling and cell death. Toxicology 2005, 208 (2), 177-192.

3. Li, C. Q.; Wogan, G. N., Nitric oxide as a modulator of apoptosis. Cancer Lett. 2005, $226(1), 1-15$.

4. Calabrese, V.; Mancuso, C.; Calvani, M.; Rizzarelli, E.; Butterfield, D. A.; Stella, A. M. G., Nitric oxide in the central nervous system: neuroprotection versus neurotoxicity. Nat. Rev. Neurosc. 2007, 8 (10), 766-775.

5. Vidwans, A. S.; Kim, S.; Coffin, D. O.; Wink, D. A.; Hewett, S. J., Analysis of the neuroprotective effects of various nitric oxide donor compounds in murine mixed cortical cell culture. J. Neurochem. 1999, 72 (5), 1843-1852.

6. $\quad$ Ridnour, L. A.; Thomas, D. D.; Switzer, C.; Flores-Santana, W.; Isenberg, J. S.; Ambs, S.; Roberts, D. D.; Wink, D. A., Molecular mechanisms for discrete nitric oxide levels in cancer. Nitric Oxide-Biol. Ch. 2008, 19 (2), 73-76.

7. Grisham, M. B.; Jourd'Heuil, D.; Wink, D. A., Nitric oxide - I. Physiological chemistry of nitric oxide and its metabolites: implications in inflammation. Am. J. Physiol. Gastrointest. Liver Physiol. 1999, 276 (2), G315-G321. 
8. Zhou, L.; Zhu, D.-Y., Neuronal nitric oxide synthase: Structure, subcellular localization, regulation, and clinical implications. Nitric Oxide-Biol. Ch. 2009, 20 (4), 223-230.

9. Hogg, N., Detection of nitric oxide by electron paramagnetic resonance spectroscopy. Free Radic. Biol. Med. 2010, 49 (2), 122-129.

10. Lalezari, P.; Lekhraj, R.; Casper, D., A method for concentrating organic dyes: Colorimetric measurements of nitric oxides and sialic acids. Anal. Biochem. 2011, $416(1), 92-99$.

11. Pluth, M. D.; Chan, M. R.; McQuade, L. E.; Lippard, S. J., Seminaphthofluorescein-Based Fluorescent Probes for Imaging Nitric Oxide in Live Cells. Inorg. Chem. 2011, 50 (19), 9385-9392.

12. Pluth, M. D.; Tomat, E.; Lippard, S. J., Biochemistry of Mobile Zinc and Nitric Oxide Revealed by Fluorescent Sensors. In Annu. Rev. Biochem., 2011; Vol. 80, pp 333-355.

13. Tinberg, C. E.; Tonzetich, Z. J.; Wang, H.; Do, L. H.; Yoda, Y.; Cramer, S. P.; Lippard, S. J., Characterization of Iron Dinitrosyl Species Formed in the Reaction of Nitric Oxide with a Biological Rieske Center. J. Am. Chem. Soc. 2010, 132 (51), 18168-18176.

14. McQuade, L. E.; Pluth, M. D.; Lippard, S. J., Mechanism of Nitric Oxide Reactivity and Fluorescence Enhancement of the NO-Specific Probe CuFL1. Inorg. Chem. 2010, 49 (17), 8025-8033.

15. McQuade, L. E.; Lippard, S. J., Fluorescence-Based Nitric Oxide Sensing by $\mathrm{Cu}(\mathrm{II})$ Complexes That Can Be Trapped in Living Cells. Inorg. Chem. 2010, 49 (16), 7464-7471.

16. Tonzetich, Z. J.; McQuade, L. E.; Lippard, S. J., Detecting and Understanding the Roles of Nitric Oxide in Biology. Inorg. Chem. 2010, 49 (14), 6338-6348.

17. Rosenthal, J.; Lippard, S. J., Direct Detection of Nitroxyl in Aqueous Solution Using a Tripodal Copper(II) BODIPY Complex. J. Am. Chem. Soc. 2010, 132 (16), 5536-5537. 
18. Pluth, M. D.; McQuade, L. E.; Lippard, S. J., Cell-Trappable Fluorescent Probes for Nitric Oxide Visualization in Living Cells. Org. Lett. 2010, 12 (10), 2318-2321.

19. McQuade, L. E.; Ma, J.; Lowe, G.; Ghatpande, A.; Gelperin, A.; Lippard, S. J., Visualization of nitric oxide production in the mouse main olfactory bulb by a celltrappable copper(II) fluorescent probe. Proc. Natl. Acad. Sci. U. S. A. 2010, 107 (19), 8525-8530.

20. McQuade, L. E.; Lippard, S. J., Fluorescent probes to investigate nitric oxide and other reactive nitrogen species in biology (truncated form: fluorescent probes of reactive nitrogen species). Curr. Opin. Chem. Biol. 2010, 14 (1), 43-49.

21. Boens, N.; Leen, V.; Dehaen, W., Fluorescent indicators based on BODIPY. Chem. Soc. Rev. 2012, 41 (3), 1130-1172.

22. Gabe, Y.; Urano, Y.; Kikuchi, K.; Kojima, H.; Nagano, T., Highly sensitive fluorescence probes for nitric oxide based on boron dipyrromethene chromophorerational design of potentially useful bioimaging fluorescence probe. J. Am. Chem. Soc. 2004, 126 (10), 3357-3367.

23. Nagano, T., Bioimaging Probes for Reactive Oxygen Species and Reactive Nitrogen Species. J. Clin. Biochem. Nutr. 2009, 45 (2), 111-124.

24. Sasaki, E.; Kojima, H.; Nishimatsu, H.; Hirata, Y.; Nagano, T., Development of near-infrared fluorescent probes for in vivo nitric oxide imaging. Nitric Oxide-Biol. Ch. 2004, 11 (1), 87-88.

25. Gabe, Y.; Ueno, T.; Urano, Y.; Kojima, H.; Nagano, T., Tunable design strategy for fluorescence probes based on 4-substituted BODIPY chromophore: improvement of highly sensitive fluorescence probe for nitric oxide. Anal. Bioanal. Chem. 2006, 386 (3), 621-626.

26. Zhang, X.; Chi, R. A.; Zou, J.; Zhang, H. S., Development of a novel fluorescent probe for nitric oxide detection: 8-(3',4'-diaminophenyl)-difluoroboradiaza-Sindacence. Spectrochimica Acta Part a-Molecular and Biomolecular Spectroscopy 2004, 60 (13), 3129-3134.

27. Huang, K. J.; Wang, H.; Guo, Y. H.; Fan, R. L.; Zhang, H. S., Spectrofluorimetric determination of trace nitrite in food products with a new fluorescent probe $1,3,5,7-$ 
tetramethyl-2,6-dicarbethoxy-8(3',4'-diaminophenyl)-difluoroboradiaza-sindacene. Talanta 2006, 69 (1), 73-78.

28. Li, J. S.; Wang, H.; Zhang, M.; Zhang, H. S., Spectrofluorimetric determination of total amount of nitrite and nitrate in biological sample with a new fluorescent probe 1,3,5,7-tetramethyl-8-(3 ',4 '-diaminophenyl)-difluoroboradiaza-s-indacence. Talanta 2003, 61 (6), 797-802.

29. Zhang, X. A.; Wang, H.; Li, J. S.; Zhang, H. S., Development of a fluorescent probe for nitric oxide detection based on difluoroboradiaza-s-indacene fluorophore. Anal. Chim. Acta 2003, 481 (1), 101-108.

30. Rodriguez-Rodriguez, R.; Simonsen, U., Measurement of Nitric Oxide and Reactive Oxygen Species in the Vascular Wall. Curr. Anal. Chem. 2012, 8 (4), 485494.

31. Mandon, J.; Hogman, M.; Merkus, J. F. M.; van Amsterdam, J.; Harren, F. J. M.; Cristescu, S. M., Exhaled nitric oxide monitoring by quantum cascade laser: comparison with chemiluminescent and electrochemical sensors. J. Biomed. Opt. 2012, $17(1)$.

32. Loudet, A.; Burgess, K., BODIPY dyes and their derivatives: Syntheses and spectroscopic properties. Chem. Rev. 2007, 107, 4891-4932.

33. Ziessel, R.; Ulrich, G.; Harriman, A., The chemistry of Bodipy: a new El Dorado for fluorescence tools. New J. Chem. 2007, 31 (4), 496-501.

34. Ulrich, G.; Ziessel, R.; Harriman, A., The chemistry of fluorescent bodipy dyes: Versatility unsurpassed. Angew. Chem. Int. Ed. 2008, 47, 1184-1201.

35. Benniston, A. C.; Copley, G., Lighting the way ahead with boron dipyrromethene (Bodipy) dyes. PCCP 2009, 11 (21), 4124-4131.

36. Zhu, S. L.; Zhang, J. T.; Vegesna, G.; Tiwari, A.; Luo, F. T.; Zeller, M.; Luck, R.; Li, H. H.; Green, S.; Liu, H. Y., Controlled Knoevenagel reactions of methyl groups of 1,3,5,7-tetramethyl BODIPY dyes for unique BODIPY dyes. RSC Adv. 2012, 2 , 404-407. 
37. Zhu, S.; Zhang, J.; Vegesna, G.; Luo, F.-T.; Green, S. A.; Liu, H., Highly WaterSoluble Neutral BODIPY Dyes with Controllable Fluorescence Quantum Yields. Org. Lett. 2011, 13 (3), 438-441.

38. Donuru, V. R.; Zhu, S. L.; Green, S.; Liu, H. Y., Near-infrared emissive BODIPY polymeric and copolymeric dyes. Polymer 2010, 51 (23), 5359-5368.

39. Zhu, S. L.; Dorh, N.; Zhang, J. T.; Vegesna, G.; Li, H. H.; Luo, F. T.; Tiwari, A.; Liu, H. Y., Highly water-soluble neutral near-infrared emissive BODIPY polymeric dyes. J. Mater. Chem. 2012, 22 (6), 2781-2790.

40. Kojima, H.; Nakatsubo, N.; Kikuchi, K.; Kawahara, S.; Kirino, Y.; Nagoshi, H.; Hirata, Y.; Nagano, T., Detection and imaging of nitric oxide with novel fluorescent indicators: Diaminofluoresceins. Anal. Chem. 1998, 70 (13), 2446-2453.

41. Shiue, T. W.; Chen, Y. H.; Wu, C. M.; Singh, G.; Chen, H. Y.; Hung, C. H.; Liaw, W. F.; Wang, Y. M., Nitric Oxide Turn-on Fluorescent Probe Based on Deamination of Aromatic Primary Monoamines. Inorg. Chem. 2012, 51 (9), 54005408 .

42. Smith, R. C.; Tennyson, A. G.; Lim, M. H.; Lippard, S. J., Conjugated polymerbased fluorescence turn-on sensor for nitric oxide. Org. Lett. 2005, 7 (16), 35733575 .

43. Itoh, Y.; Ma, F. H.; Hoshi, H.; Oka, M.; Noda, K.; Ukai, Y.; Kojima, H.; Nagano, T.; Toda, N., Determination and bioimaging method for nitric oxide in biological specimens by diaminofluorescein fluorometry. Anal. Biochem. 2000, 287 (2), 203209. 


\section{Chapter 3 : pH-Activatable Near-Infrared Fluorescent Probes for Detection of Lysosomal pH inside Living Cells ${ }^{2}$}

Giri Vegesna, ${ }^{a}$ Jagadeesh Janjanam, ${ }^{\mathrm{a}}$ Jianheng $\mathrm{Bi}^{\mathrm{a}}{ }^{\mathrm{a}}$ Fen-Tair Luo ${ }^{\mathrm{b}}$, Connor Olds, ${ }^{\mathrm{a}}$ Ashutosh Tiwari* and Haiying Liu ${ }^{\mathrm{a} *}$

aDepartment of Chemistry, Michigan Technological University, Houghton, MI 49931

${ }^{\mathrm{b}}$ Institute of Chemistry, Academia Sinica, Taipei, Taiwan 11529, Republic of China

${ }^{2}$ This chapter is currently under review for Journal of materials chemistry $B$. 


\subsection{Abstract}

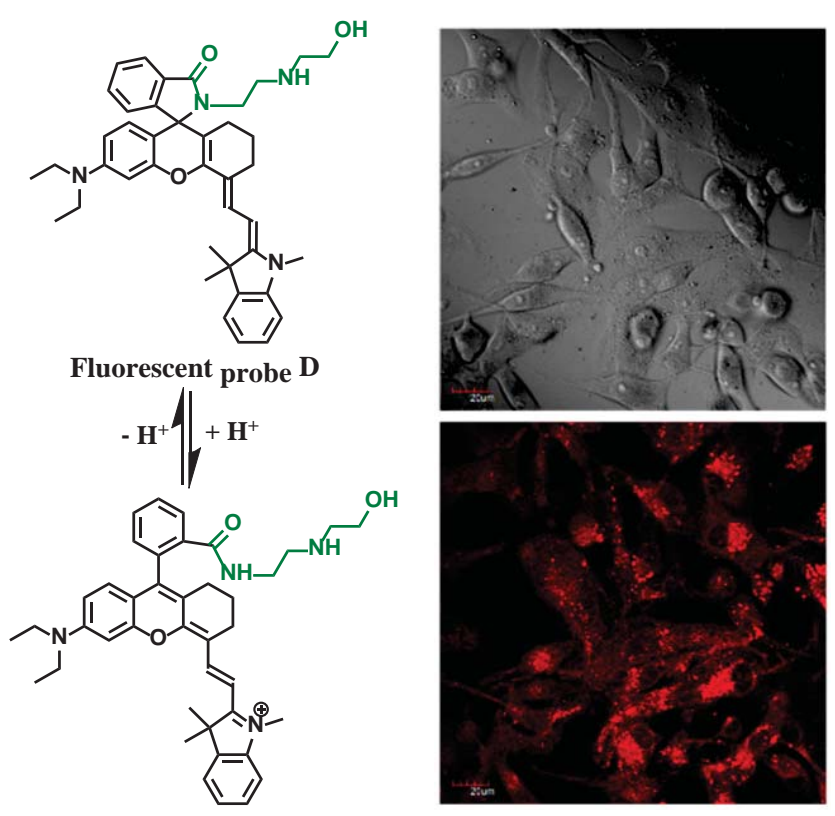

Figure 3.1. Spirocyclic ring closing and opening forms of probe D and their cell images

Four near-infrared fluorescent probes (A, B, C and D) have been synthesized, characterized, and evaluated for detection of lysosomal $\mathrm{pH}$ inside living cells. The fluorescent probes display highly sensitive and selective fluorescent response to acidic $\mathrm{pH}$ as the acidic $\mathrm{pH}$ results in drastic structural changes from spirocyclic (non-fluorescent) forms to ring-opening (fluorescent) forms of the fluorescent probes. The fluorescence intensities of the fluorescent probes (B, C and D) increase significantly by more than 200fold from $\mathrm{pH} 7.4$ to 4.2 . The fluorescent probe D bearing N-(2-hydroxyethyl) ethylene amide residue possesses advantages of high sensitivity, brilliant photostability, good cell membrane permeability, strong $\mathrm{pH}$ dependence and low auto-fluorescence background. It has been successfully applied to selectively stain lysosomes and detect lysosomal $\mathrm{pH}$ changes inside normal endothelial and breast cancer cells.

\subsection{Introduction}

Intracellular $\mathrm{pH}$, known as $\mathrm{pHi}$, plays a pivotal role in cell function and regulation such as cell volume regulation, vesicle trafficking, cellular metabolism, cell membrane polarity, cellular signaling, cell activation, growth, proliferation and apoptosis. ${ }^{1-4}$ Abnormal $\mathrm{pH}$ values in organelles are associated with many diseases such as cancer and Alzheimer's. 5, 6 
A low intra-compartmental $\mathrm{pH}$ in organelles functions to denature proteins or activate enzymes that are normally inactive around neutral $\mathrm{pH}^{7}$ For example, the membrane around lysosome at $\mathrm{pH} 4.5$ - 5.0 enables digestive enzymes to degrade proteins, DNA, RNA, polysaccharides, lipids, viruses and bacteria. ${ }^{4,7,8}$ Significant disruptive changes in the lysosomal $\mathrm{pH}$ can lead to lysosome malfunction and consequently result in lysosomal storage diseases. ${ }^{9}$ Therefore, it is very important to precisely monitor lysosomal $\mathrm{pH}$ inside living cells in order to investigate cellular functions and understand physiological and pathological processes. A variety of techniques such as microelectrodes, nuclear magnetic resonance, ${ }^{10}$ absorbance spectroscopy, and fluorescence imaging and spectroscopy $y^{11-22}$ have been developed to measure intracellular $\mathrm{pH}$. Fluorescence spectroscopy using $\mathrm{pH}-$ sensitive fluorescent probes is becoming one of the most powerful tools for monitoring intracellular $\mathrm{pH}$, and possesses many technical and practical advantages over other methods because it can detect the intracellular $\mathrm{pH}$ of intact cells and subcellular regions with operational simplicity, high sensitivity, and excellent spatial and temporal resolution. ${ }^{11-21}$ Although several fluorescent probes for $\mathrm{pH}$ have been developed, ${ }^{23}$ only a few of them have been applied to detect lysosomal $\mathrm{pH}$ inside living cells. Most fluorescent probes including the commercial ones take advantages of lysosomotropism where their weak bases of tertiary amine groups help the probes selectively accumulate in acidic lysosomes through the protonation of the amine groups in a cellular acidic environment. ${ }^{18}$ Their $\mathrm{pH}$ sensitivity primarily results from protonation of ionizable tertiary amine groups on the fluorophores in acidic lysosomes enhancing the probe fluorescence through the suppression of photo-induced electron transfer from the tertiary amine to the probe fluorophores. ${ }^{15}$ The potential drawback for these fluorescent probes is their broad $\mathrm{pH}$ response and relative high fluorescent background at $\mathrm{pH}$ 7.4. In order to address the fluorescent background issue, a few fluorescent probes for $\mathrm{pH}$ based on fluorescein and Rhodamine dyes have been developed to take advantages of low fluorescence background at $\mathrm{pH} 7.4$ because spirolactam rings of the fluorophores exists in the "ring-closed" state at $\mathrm{pH}$ 7.4. ${ }^{12,13,20,24}$ However, some fluorescent probes can result in cell damage because of their short absorption and emission wavelengths with less than $600 \mathrm{~nm}$. In this paper, we report four near-infrared fluorescent probes (A, B, C and D) with spirocyclic structures 
which are highly sensitive to the $\mathrm{pH}$ changes of the solutions. Under neutral or basic conditions, the fluorescent probes retain the spirocyclic form that is non-fluorescent and colorless. Acidic environment effectively triggers ring opening of the spirocylic form in fluorescent probes, and results in strong fluorescence (Scheme 3.1). Three sensitive nearinfrared fluorescent probes $(\mathrm{B}, \mathrm{C}$ and $\mathrm{D})$ have been used to detect lysosomal $\mathrm{pH}$ inside living cells with advantages of deep tissue light penetration and low autofluorescence. The fluorescent probes display extremely weak fluorescence at the extracellular $\mathrm{pH}$ at 7.4 and become strongly fluorescent at the intracellular lysosomal $\mathrm{pH}$ at 4.5.

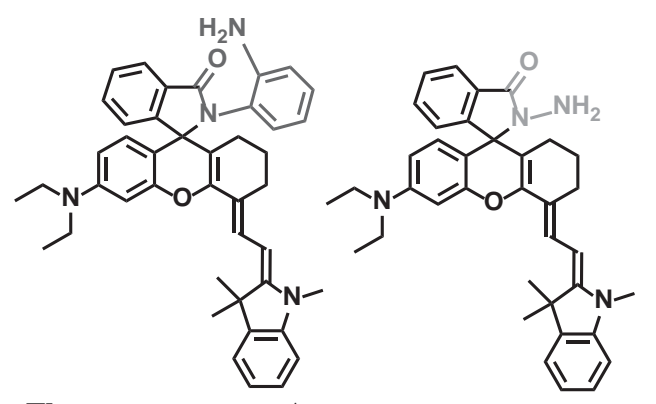

Fluorescent probe $A$ Fluorescent probe $B$

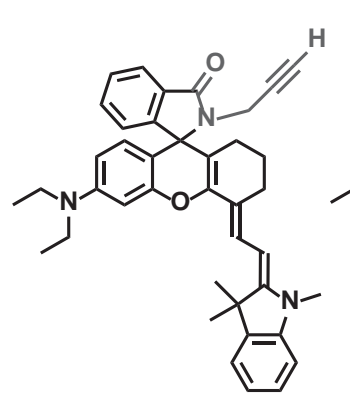

Fluorescent probe $\mathrm{C}$<smiles>[CH2-][C@H]1C=C[C@H]1[IH+]</smiles><smiles>[InH+][C@@H]1C=C[C@@H]1[I-]</smiles>

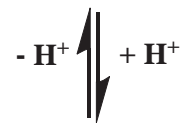

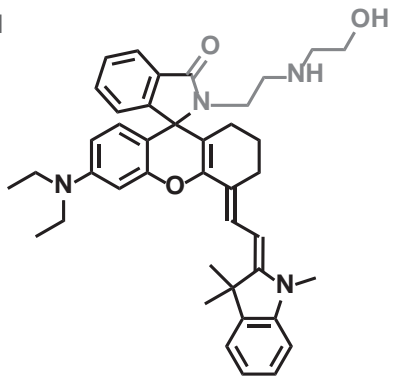

Fluorescent probe $D$

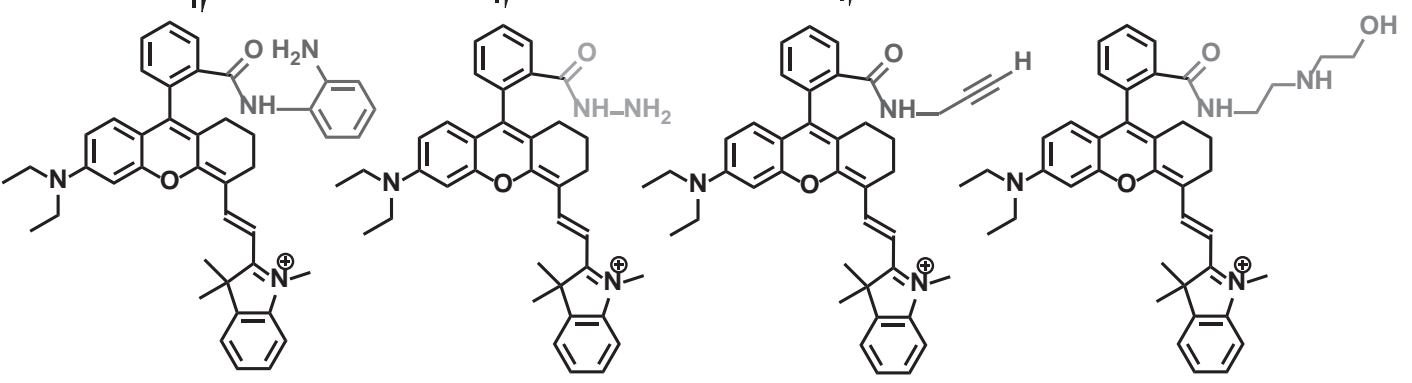

Scheme 3.1. Spirocyclic ring closing and opening forms of near-infrared fluorescent probes upon $\mathrm{pH}$ changes.

\subsection{Experimental Section}

\subsubsection{Instrumentation.}

${ }^{1} \mathrm{H}$ NMR and ${ }^{13} \mathrm{C}$ NMR spectra were obtained by using a $400 \mathrm{MHz}$ Varian Unity Inova NMR spectrophotometer instrument. ${ }^{1} \mathrm{H}$ and ${ }^{13} \mathrm{C}$ NMR spectra were recorded in $\mathrm{CDCl}_{3}$, 
chemical shifts $(\delta)$ are given in ppm relative to solvent peaks $\left({ }^{1} \mathrm{H}: \delta 7.26 ;{ }^{13} \mathrm{C}: \delta 77.3\right)$ as internal standard. HRMS were measured with fast atom bombardment (FAB) ionization mass spectrometer, double focusing magnetic mass spectrometer or matrix assisted laser desorption/ionization time of flight mass spectrometer. Infrared (IR) spectra were obtained in the 400-4000 $\mathrm{cm}^{-1}$ range using Perkin Elmer Spectrum One FTIR spectrometer. Absorption spectra were taken on a Perkin Elmer Lambda 35 UV/VIS spectrometer. Fluorescence spectra were recorded on a Jobin Yvon Fluoromax-4 spectrofluorometer. Fluorescence spectra of fluorescent probes were measured by using standard 1-cm pathlength fluorescence quartz cuvette at room temperature. All samples were scanned with increments of $1 \mathrm{~nm}$. Each spectrum for absorbance and fluorescence was measured at room temperature. $5 \mu \mathrm{M}$ fluorescent probe solution was used every single time under different $\mathrm{pH}$ environment. The citric acid sodium phosphate buffer solution was prepared freshly. The excitation wavelength at $670 \mathrm{~nm}$ was used to excite all fluorescent dyes.

\subsubsection{Materials}

Unless otherwise indicated, all reagents and solvents were obtained from commercial suppliers and used without further purification. Air- and moisture-sensitive reactions were conducted in oven-dried glassware using a standard Schlenk line or drybox techniques under an inert atmosphere of dry nitrogen.

Fluorescent probe A: When phosphorous oxychloride ( $0.68 \mathrm{~g}, 4.4 \mathrm{mmol})$ was added to the compound $5(0.5 \mathrm{~g}, 0.89 \mathrm{mmol})$ in 1,2-dichloroethane $(20 \mathrm{~mL})$ under a nitrogen atmosphere, the resulting reaction mixture was refluxed for 4 hours. After the solvent was removed under reduced pressure, dry acetonitrile $(30 \mathrm{~mL})$ was added to the reaction residue. When 1,2-diaminobenzene $(6)(0.38 \mathrm{~g}, 3.5 \mathrm{mmol})$ and triethylamine $(0.5 \mathrm{~mL})$ was added to the reaction solution, the reaction mixture was stirred at room temperature for overnight. After the solvent was removed under reduced pressure, the reaction residue was dissolved in dichloromethane $(50 \mathrm{~mL})$, washed it with water $(2 \times 20 \mathrm{~mL})$ and brine solution $(2 \times 20 \mathrm{~mL})$, respectively. Organic layer was collected, dried over anhydrous sodium sulfate, filtered and concentrated under reduced pressure. The crude compound was purified by flash column chromatography using EtOAc/hexane (50/50) to yield yellow 
crystal $(0.34 \mathrm{~g}, 60 \%) .{ }^{1} \mathrm{H}$ NMR $\left(400 \mathrm{MHz}, \mathrm{CDCl}_{3}\right): \delta 7.97-7.95(\mathrm{~d}, J=8.0 \mathrm{~Hz}, 1 \mathrm{H}), 7.54-$ 7.45 (m, 2H), 7.34-7.31 (d, J = 12.0 Hz, 1H), 7.24-7.22 (d, $J=8.0 \mathrm{~Hz}, 1 \mathrm{H}), 7.16-7.11(\mathrm{~m}$, 2H), 6.99-6.95 (t, $J=8.0 \mathrm{~Hz}, 1 \mathrm{H}), 6.83-6.80(\mathrm{t}, J=8.0 \mathrm{~Hz}, 1 \mathrm{H}), 6.66-6.52(\mathrm{~m}, 5 \mathrm{H}), 6.31-$ $6.24(\mathrm{~m}, 2 \mathrm{H}), 5.34-5.31(\mathrm{~d}, J=12.0 \mathrm{~Hz}, 1 \mathrm{H}), 3.36-3.31$ (q, $J=8.0 \mathrm{~Hz}, 4 \mathrm{H}), 3.11(\mathrm{~s}, 3 \mathrm{H})$, 2.63-2.57 (m, 1H), 2.38-2.30 (m, 1H), 2.15-2.04 (m, 1H), 1.66-1.61 (m, 7H). 1.37-1.25 (m, $2 \mathrm{H}), 1.18-1.14$ (t, $J=8.0 \mathrm{~Hz}, 6 \mathrm{H}) .{ }^{13} \mathrm{C}$ NMR $\left(100 \mathrm{MHz}, \mathrm{CDCl}_{3}\right): \delta 167.10,157.65,153.41$, $152.40,148.97,148.32,145.56,144.14,139.06,132.66,131.63,128.79,128.42,127.88$, $127.38,123.96,123.54,123.47,121.71,120.85,119.42,119.34,118.93,118.10,108.57$, $105.85,104.38,98.24,92.24,70.89,45.60,44.54,29.50,29.29,28.66,28.36,25.52,24.60$, 23.98, 22.28, 12.76. IR ( $\left.\mathrm{cm}^{-1}\right): 3346.38,2968.95,2928.01,1681.57,1619.38,1592.07$, $1493.12,1354.13,1316.93,1263.72,1216.24,1192.80,1126.33,1078.00,1019.85$, 930.06, 735.45, 702.26. HRMS (ESI) calcd for $\mathrm{C}_{43} \mathrm{H}_{45} \mathrm{~N}_{4} \mathrm{O}_{2}[\mathrm{M}+\mathrm{H}]^{+}, 649.3542$; found, 649.3559 .

Fluorescent probe B: After compound $5(0.30 \mathrm{~g}, 0.53 \mathrm{mmol})$ was dissolved in dry dichloromethane $(30 \mathrm{~mL})$ under nitrogen atmosphere at room temperature, dicyclohexylcarbodiimide (DCC) $(0.10 \mathrm{~g}, 0.53 \mathrm{mmol})$, and 4-dimethylaminopyridine $(0.007 \mathrm{~g}, 0.06 \mathrm{mmol})$ were added to the reaction mixture at room temperature. When hydrazine hydrate $(0.2 \mathrm{~mL}, 6.25 \mathrm{mmol})$ was added to the mixture, the reaction mixture was stirred for 60 minutes at room temperature. Workup procedure was the same as that for fluorescent probe $\mathbf{A}$. The crude compound was purified by flash column chromatography using EtOAc/Hexane (50/50) to get a yellow solid (0.15 g, 50\%). ${ }^{1} \mathrm{H}$ NMR (400 MHz, $\left.\mathrm{CDCl}_{3}\right): \delta$ 7.89-7.87 (d, $\left.J=8.0 \mathrm{~Hz}, 1 \mathrm{H}\right), 7.49-7.40(\mathrm{~m}, 3 \mathrm{H}), 7.19-7.13(\mathrm{~m}, 3 \mathrm{H}), 6.86-6.79$ (m, 1H), 6.60-6.59 (m, 1H), 6.39-6.26 (m, 3H), 5.37-5.34 (d, $J=12.0 \mathrm{~Hz}, 1 \mathrm{H}), 3.64$ (brs, $2 \mathrm{H}), 3.36-3.31(\mathrm{q}, J=8.0 \mathrm{~Hz}, 4 \mathrm{H}) 3.13(\mathrm{~s}, 3 \mathrm{H}), 2.60-2.43(\mathrm{~m}, 2 \mathrm{H}), 1.93-1.89(\mathrm{~m}, 1 \mathrm{H}), 1.71-$ $1.69(\mathrm{~m}, 6 \mathrm{H}), 1.36-1.24(\mathrm{~m}, 3 \mathrm{H}), 1.18-1.14(\mathrm{t}, J=8.0 \mathrm{~Hz}, 6 \mathrm{H}) .{ }^{13} \mathrm{C} \mathrm{NMR}(100 \mathrm{MHz}$, $\left.\mathrm{CDCl}_{3}\right): \delta 166.50,157.96,153.45$ 149.82, 148.99, 148.75, 145.55, 139.05, 132.50, 131.01, 128.42 , 127.91, 123.66, 123.17, 121.73, 120.32, 119.86, 119.49, 108.61, 105.88, 104.29, $102.93,98.17,92.22,68.02,49.20,45.64,44.59,34.19,30.26,30.14,29.33,28.58,28.50$, 25.87, 25.55, 25.19, 23.20, 22.50, 12.78. IR ( $\left.\mathrm{cm}^{-1}\right): 2929.64,1700.81,1622.93,1595.03$, 
1517.70, 1493.73, 1318.14, 1264.97, 1128.16, 735.27, 702.01. HRMS (ESI) calcd for $\mathrm{C}_{37} \mathrm{H}_{41} \mathrm{~N}_{4} \mathrm{O}_{2}[\mathrm{M}+\mathrm{H}]^{+}$, 573.3229; found, 573.3247.

Fluorescent probe C: When compound $5(0.30 \mathrm{~g}, 0.53 \mathrm{mmol})$ was dissolved in dry dichloromethane $(20 \mathrm{~mL})$ under nitrogen atmosphere at room temperature, N-hydroxysuccinimide (0.08 g, $0.69 \mathrm{mmol})$, dicyclohexylcarbodiimide (DCC) $(0.11 \mathrm{~g}, 0.53 \mathrm{mmol})$ were added to the reaction mixture sequentially. After the reaction for 30 minutes, propargylamine $(0.04 \mathrm{~g}, 0.80 \mathrm{mmol})$ was added through a syringe under nitrogen atmosphere and the reaction mixture was stirred at room temperature for 2 hours. After the reaction mixture was washed with water $(2 \times 20 \mathrm{~mL})$, the organic layer was collected, dried over anhydrous sodium sulfate and filtered. The filtrate was concentrated under reduced pressure. The crude compound was purified by flash column chromatography using EtOAc/Hexane (25/75) to afford pale yellow syrupy compound $(0.19 \mathrm{~g}, 60 \%) .{ }^{1} \mathrm{H}$ NMR (400 MHz, $\left.\mathrm{CDCl}_{3}\right): \delta 7.89-7.87(\mathrm{~d}, J=8.0 \mathrm{~Hz}, 1 \mathrm{H}), 7.50-7.39(\mathrm{~m}, 3 \mathrm{H}), 7.20-7.14(\mathrm{~m}, 3 \mathrm{H})$, 6.88-6.79 (m, 1H), 6.65-6.53 (m, 1H), 6.34-6.24 (m, 3H), 5.44-5.27 (m, 1H), 4.24-4.19 (dd, $J=16.0,4.0 \mathrm{~Hz}, 1 \mathrm{H}), 3.76-3.71(\mathrm{dd}, J=16.0,4.0 \mathrm{~Hz}, 1 \mathrm{H}), 3.36-3.31(\mathrm{q}, J=8.0 \mathrm{~Hz}$, 4H) 3.20-3.03 (brs, $3 \mathrm{H}), 2.67-2.30(\mathrm{~m}, 2 \mathrm{H}), 2.01-1.95(\mathrm{~m}, 1 \mathrm{H}), 1.75-1.70(\mathrm{~m}, 6 \mathrm{H}), 1.61-$ $1.24(\mathrm{~m}, 4 \mathrm{H}), 1.18-1.15(\mathrm{t}, J=8.0 \mathrm{~Hz}, 6 \mathrm{H}) .{ }^{13} \mathrm{C} \mathrm{NMR}\left(100 \mathrm{MHz}, \mathrm{CDCl}_{3}\right): \delta 167.79,153.26$, 148.97, 132.66, 131.56, 128.70, 128.36, 127.97, 123.67, 123.26, 121.76, 119.55, 108.67, $105.93,104.59,97.99,79.28,70.43,45.66,44.60,28.66,25.56,24.62,23.43,22.19,12.81$. IR $\left(\mathrm{cm}^{-1}\right): 3303.82,2981.38,1699.76,1501.13,1393.66,1368.38,1250.46,1146.75$, 1049.46, 855.42, 736.62, 702.53. HRMS (FAB) calcd for $\mathrm{C}_{40} \mathrm{H}_{42} \mathrm{~N}_{3} \mathrm{O}_{2}[\mathrm{M}]^{+}, 596.3277$; found, 596.3276 .

Fluorescent probe D: After 1,1'-carbonyldiimidazole (9) (0.06 g, $0.4 \mathrm{mmol}$ ) was added to the solution of compound $5(0.15 \mathrm{~g}, 0.26 \mathrm{mmol})$ in anhydrous dichloromethane $(20 \mathrm{~mL})$ under nitrogen atmosphere, the reaction mixture was stirred at room temperature for 4 hours. After the complete consumption of compound 5 (confirmed by TLC), N-(2hydroxyethyl)ethylenediamine $(0.05 \mathrm{~mL}, 0.53 \mathrm{mmol})$ was added to the mixture, the reaction mixture was further stirred overnight. After work-up, the organic layer was washed with water $(2 \times 30 \mathrm{~mL})$ and brine solution $(2 \times 30 \mathrm{~mL})$, respectively, dried over anhydrous sodium sulfate, and filtered. The filtrate was concentrated under reduced 
pressure. The crude compound was purified by preparative TLC plate with dichloromethane/EtOH (20/1) to afford a brown solid (0.09 g, 52\%). ${ }^{1} \mathrm{H}$ NMR (400 MHz, $\left.\mathrm{CDCl}_{3}\right): \delta$ 7.81-7.79 (d, $\left.J=8.0 \mathrm{~Hz}, 1 \mathrm{H}\right), 7.49-7.34(\mathrm{~m}, 3 \mathrm{H}), 7.16-7.09(\mathrm{~m}, 3 \mathrm{H}), 6.81-6.77$ $(\mathrm{t}, J=8.0 \mathrm{~Hz}, 1 \mathrm{H}), 6.57-6.55(\mathrm{~d}, J=8.0 \mathrm{~Hz}, 1 \mathrm{H}), 6.35-6.23(\mathrm{~m}, 3 \mathrm{H}), 5.37-5.34(\mathrm{~d}, J=12.0$ $\mathrm{Hz}, 1 \mathrm{H}), 3.53-3.26$ (m, 8H), 3.10 (s, 3H), 2.67-2.38 (m, 6H), 1.72-1.60 (m, 7H), 1.39-1.23 $(\mathrm{m}, 3 \mathrm{H}), 1.16-1.12(\mathrm{t}, J=8.0 \mathrm{~Hz}, 6 \mathrm{H}) \cdot{ }^{13} \mathrm{C} \mathrm{NMR}\left(100 \mathrm{MHz}, \mathrm{CDCl}_{3}\right): \delta 169.13,157.96$, $152.96,151.81,148.90,148.26,145.50,138.99,132.42$, 132.04, 128.60, 128.36, 127.93, $123.62,123.01,121.72,120.27,119.87,119.55,108.66,107.87,105.93,105.46,103.60$, $97.95,92.24,67.24,61.06,51.17,48.14,45.65,44.57,40.03,30.58,30.16,29.89$, 29.32, 28.71, 28.52, 25.55, 23.25, 22.44, 12.78. IR ( $\left.\mathrm{cm}^{-1}\right): 3364.27,2968.80,2927.21,1682.60$, $1621.06,1592.96,1515.14,1492.83,1466.55,1352.63,1316.87,1264.54,1214.76$, 1192.54, 1125.75, 1077.46, 929.65, 817.77, 733.88, 701.34. HRMS (ESI) calcd for $\mathrm{C}_{41} \mathrm{H}_{49} \mathrm{~N}_{4} \mathrm{O}_{3}[\mathrm{M}+\mathrm{H}]^{+}, 645.3804$; found, 645.3823 .

\subsubsection{Cell culture and confocal fluorescence imaging}

Breast cancer (MDA-MB-231) and normal endothelial (HUVEC-C) cell lines were obtained from ATCC. The cells were cultured according to the published procedures. ${ }^{25}$ Briefly, the cells were plated on 12-well culture plates or $35 \mathrm{~mm}$ glass bottom culture dishes (MatTek Corp.) at a density of 1 X $10^{5}$ cells/ $\mathrm{mL}$ for live cell imaging. After 24-h incubation at $37{ }^{\circ} \mathrm{C}$ in $5 \% \mathrm{CO}_{2}$ incubator, the media was removed and cells were rinsed with 1X PBS. Fresh serum free media with 5 or $20 \mu \mathrm{M}$ of fluorescent probes $\mathbf{B}, \mathbf{C}$ and $\mathbf{D}$ were added and incubated for 2 hours. Live cell imaging was performed with inverted fluorescence microscope (Model AMF-4306; $E_{\text {VOS }}$, AMG) for initial dye concentration standardization. The final cell images were obtained with confocal laser scanning microscope (Olympus FV1000) with excitation wavelengths at $405 \mathrm{~nm}$ for Hoechst 33342 (Sigma-Aldrich), at $488 \mathrm{~nm}$ for LysoSensor Green DND-189 (Invitrogen), and at $635 \mathrm{~nm}$ for fluorescent probes B, C and D. The fluorescence images were obtained at $60 \mathrm{X}$ magnification and the exposure times for each laser were kept constant for each image series. 


\subsection{Results and Discussion}

\subsubsection{Design and synthesis of near-infrared fluorescent probes for $\mathrm{pH}$}

Fluorescent dye (5) ((E)-2-(2-(9-(2-carboxyphenyl)-6-(diethylamino)-2,3-dihydro-1Hxanthen-4-yl)vinyl)-1,3,3-trimethyl-3H-indol-1-ium perchlorate) was chosen as a nearinfrared fluorophore to prepare near-infrared fluorescent probes for lysosomal $\mathrm{pH}$ in living cells because of its advantageous photophysical properties including a large absorption extinction coefficient $(1.4 \times 105 \mathrm{M}-1 \mathrm{~cm}-1)$, high fluorescence quantum yield $(41 \%$ in methanol) with near-infrared emission peak at $720 \mathrm{~nm}$, good photostability and chemical stability. It displays absorption maximum peak at $710 \mathrm{~nm}$ due to $\mathrm{S} 0 \rightarrow \mathrm{S} 1$ transition, a shoulder peak at $650 \mathrm{~nm}$, and near-infrared emission peak at $731 \mathrm{~nm}$ in ethanol mixed solution (please see Figures B.24 and B.25 in supporting information). Fluorescent dye (5) was prepared by condensation of 2-(4-(diethylamino)-2-hydroxybenzoyl)benzoic acid (1) with cyclohexanone (2) in acidic conditions, yielding 9-(2-carboxyphenyl)-6(diethylamino)-1,2,3,4-tetrahydroxanthylium perchlorate (3), and followed by condensation of compound 3 with Fisher's aldehyde (4) in acetic anhydride at $50{ }^{\circ} \mathrm{C}$ (Scheme 3.2).26 Near-infrared fluorescent probes are readily synthesized from fluorescent dye 5 through one- or two-step procedures. Fluorescent probe A was synthesized by treating fluorescent dye 5 with phosphoryl chloride (POCl3), and followed by further reaction with 1,2-diaminobenzene. Fluorescent probe B was prepared by coupling fluorescent dye 5 with dicyclohexylcarbodiimide (DCC) and 4-dimethylaminopyridine (DMAP) in dichloromethane for 30 minutes, and followed by further reaction with hydrazine hydrate for one hour. Fluorescent probe $\mathrm{C}$ was synthesized by coupling fluorescent dye 5 with $\mathrm{N}$-hydroxysuccinimide in the presence of dicyclohexylcarbodiimide (DCC) for 30 minutes, and followed by further reaction with propargylamine (Scheme 3.2). Fluorescent probe $\mathrm{D}$ was prepared by reacting fluorescent dye 5 with 1,1'carbonyldiimidazole (9) in dry dichloromethane for four hours, and followed by further reaction with $\mathrm{N}$-(2-hydroxyethyl)ethylenediamine for overnight. Introduction of N-(2hydroxyethyl)ethylenediamine to fluorescent dye 5 is expected to enhance hydrophilic property of fluorescent probe and facilitate selective accumulation of fluorescent probe D 
in lysosome in living cells via protonation of the secondary amine in an acidic environment at $\mathrm{pH} 4.5$ inside lysosome.

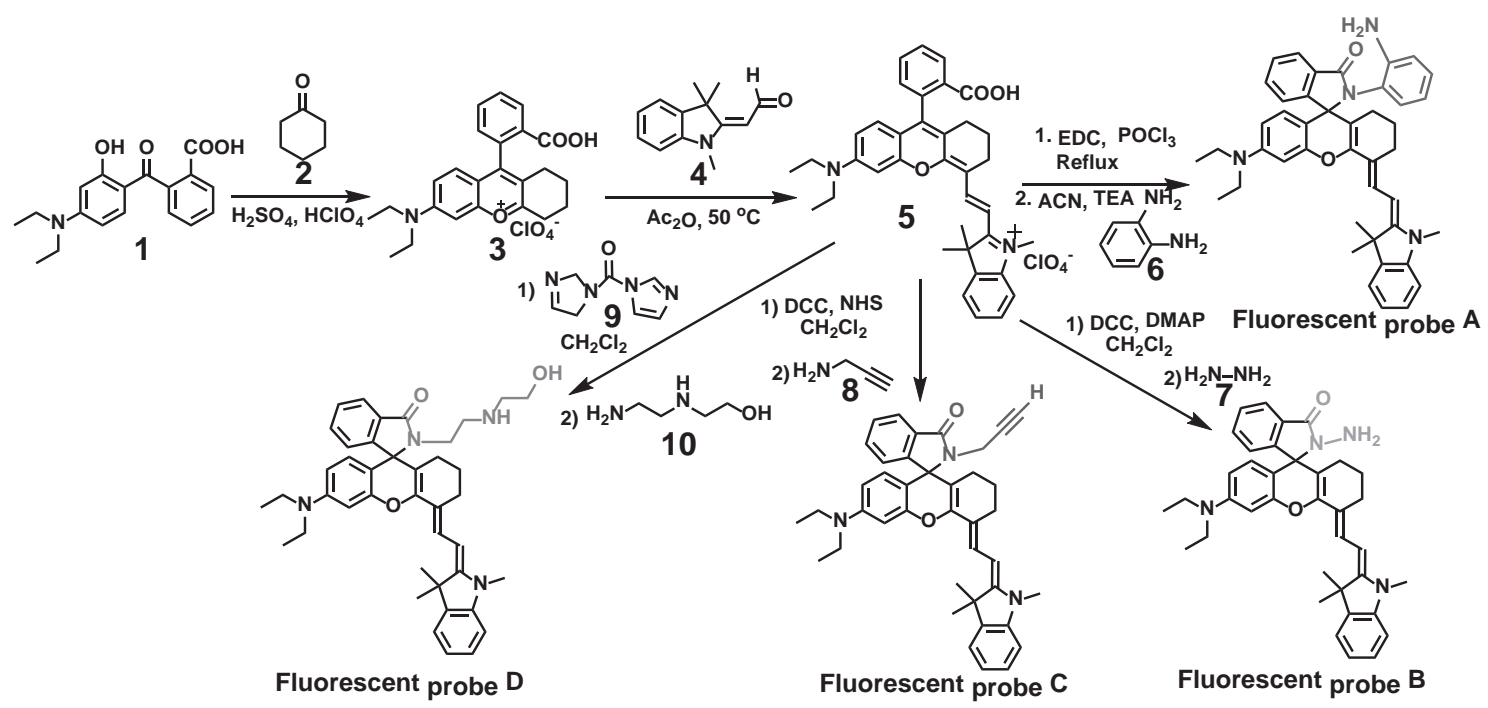

Scheme 3.2. Synthetic routes to near-infrared fluorescent probe for $\mathrm{pH}$.

\subsubsection{Optical responses of fluorescent probes to $\mathrm{pH}$}

We investigated effect of $\mathrm{pH}$ on absorption spectra of the fluorescent probes. Fluorescent probe A displays a strong absorption peak at $379 \mathrm{~nm}$, a moderate absorption at $479 \mathrm{~nm}$, and an extremely weak absorption peak at $720 \mathrm{~nm}$ in $40 \mathrm{mM}$ citrate-phosphate buffer solution ( $\mathrm{pH}$ 6.9) containing 40\% ethanol (Figure 3.2). Gradual decreases of $\mathrm{pH}$ from $\mathrm{pH}$ 7.0 to $\mathrm{pH} 4.0$ results in significant absorbance enhancement at $720 \mathrm{~nm}$ accompanying with a shoulder peak at $663 \mathrm{~nm}$ (Figure 3.2), indicating that the spirolactam ring of the fluorophore was opened, leading to significant extension of $\pi$-conjugation of the fluorophore. Consequently, the solution of the fluorescent probe A displays distinct color changes from colorless to green along with the $\mathrm{pH}$ titration from 6.9 to 4.0. However, further decreases of $\mathrm{pH}$ below 2.0 causes decrease in the absorbance of fluorescent probe A at $720 \mathrm{~nm}$ (Figure 3.2). This may be due to charge imbalance of the fluorophore as the tertiary amine group of the fluorophore becomes protonated at extremely low $\mathrm{pH}$ (Scheme 3.3). Fluorescent probe $\mathbf{A}$ displays full fluorescent reversible responses between $\mathrm{pH} 3.0$ and 7.2 when it is treated with acid or base. The similar $\mathrm{pH}$ effect on absorbance of 
fluorescent probes B, C and D were also observed (Figure 3.3, and Figures B.17-B.19 in supporting information).

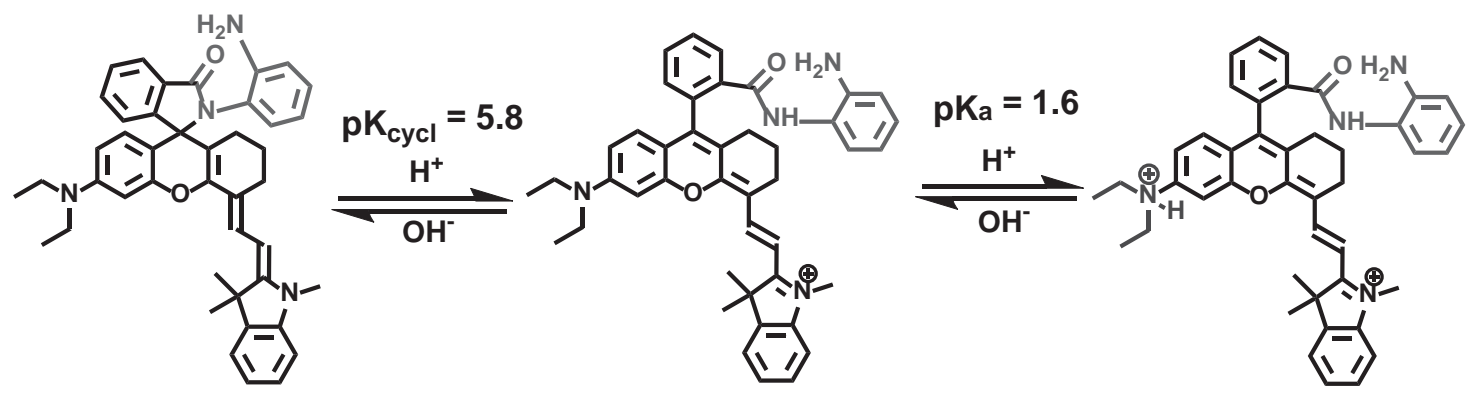

Fluorescent probe $\mathrm{A}$

Scheme 3.3. Chemical structures of fluorescent probe $\mathbf{A}$ at different $\mathrm{pH}$ values.
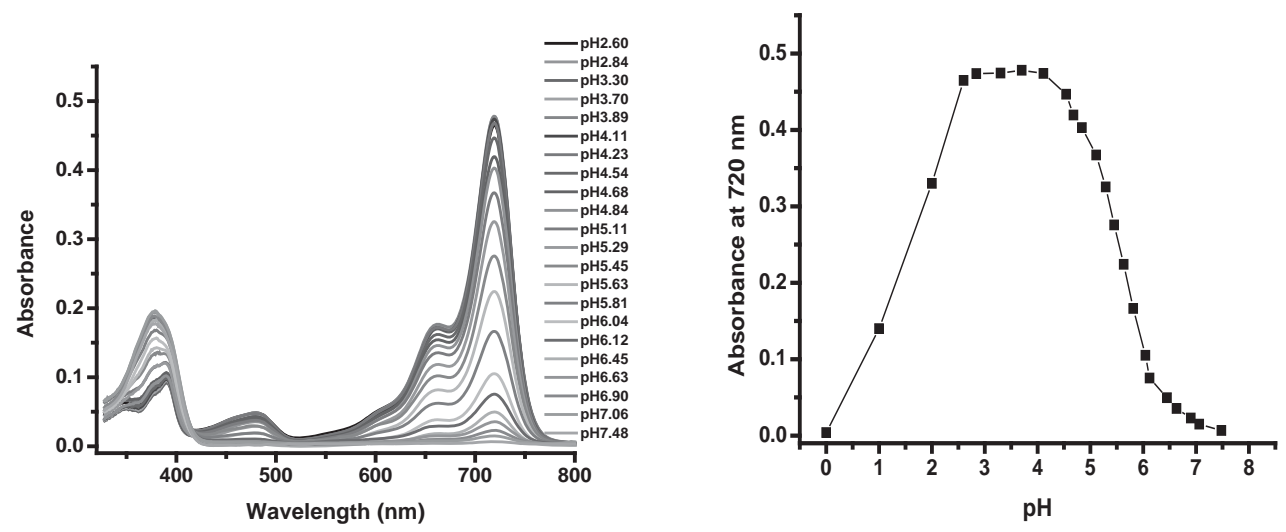

Figure 3.2. Absorption spectra of $5 \mu \mathrm{M}$ fluorescent probe $\mathbf{A}$ at different $\mathrm{pH}$ values (left) and effect of $\mathrm{pH}$ on absorbance of the fluorescent probe $\mathbf{A}$ at $718 \mathrm{~nm}$ (right). 

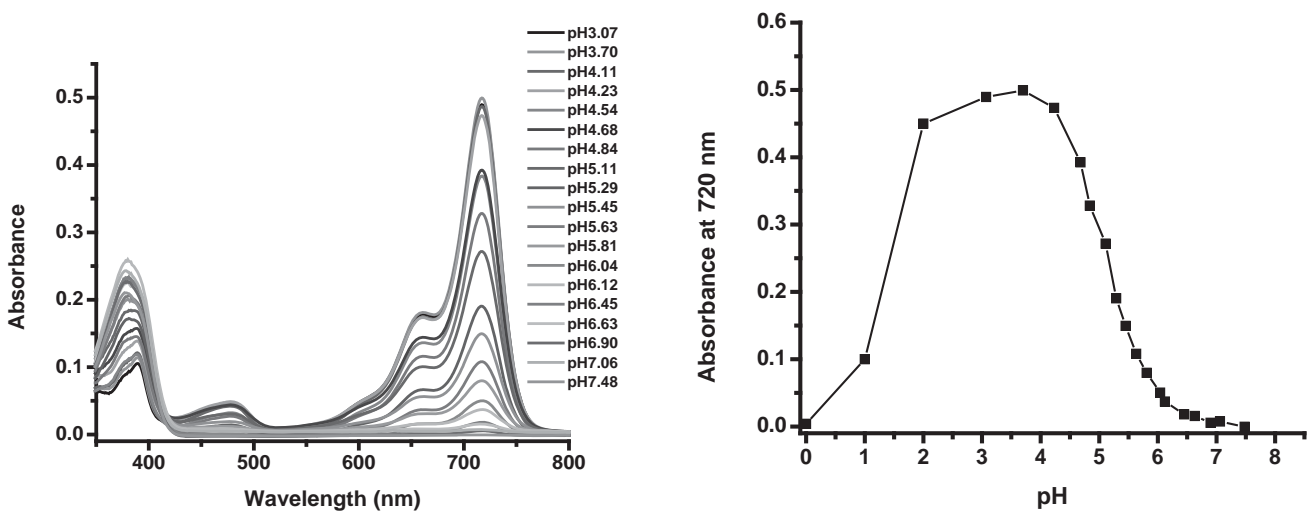

Figure 3.3. Absorption spectra of $5 \mu \mathrm{M}$ fluorescent probe $\mathbf{D}$ at different $\mathrm{pH}$ values (left) and effect of $\mathrm{pH}$ on absorbance of the fluorescent probe $\mathbf{D}$ at $718 \mathrm{~nm}$ (right).

In order to evaluate fluorescent probes for $\mathrm{pH}$ sensing application, we investigated $\mathrm{pH}$ effect on fluorescence intensity of $5 \mu \mathrm{M}$ fluorescent probes in $40 \mathrm{mM}$ citrate-phosphate buffer solution containing 40\% ethanol. Figure 3.4 displays the fluorescence spectra of fluorescent probe $\mathbf{A}$ at different $\mathrm{pH}$ values. The probe was non-fluorescent when the buffer $\mathrm{pH}$ is greater than 7.4. However, gradual decrease of $\mathrm{pH}$ from $\mathrm{pH} 7.4$ to $\mathrm{pH} 4.0$ results in appearance of a new fluorescence peak at $743 \mathrm{~nm}$, and significantly enhances fluorescence peak intensity. There is more than 71-fold increase in the fluorescence intensity of fluorescent probe $\mathbf{A}$ at $743 \mathrm{~nm}$ with $\mathrm{pH}$ decrease from 7.4 to 4.1, indicating the probe is very sensitive to acidic $\mathrm{pH}$ because of the $\mathrm{H}^{+}$-induced spirolactam ring opening of the fluorophore. The $\mathrm{pK}_{\mathrm{cycl}}$ value of the probe $\mathbf{A}$ is 5.8 related to spirolactam ring opening, which was obtained according to the Henderson-Hasselbach-type mass action equation. The fluorescence intensity of the probe $\mathbf{A}$ displayed linear responses to $\mathrm{pH}$ values in the range from 4.9 to 6.6. In addition, it showed excellent reversible responses to $\mathrm{pH}$ between 4.0 and 7.4. Compared with fluorescent probe $\mathbf{A}$, fluorescent probes $\mathbf{B}, \mathbf{C}$ and $\mathbf{D}$ exhibit much more sensitive fluorescent responses to $\mathrm{pH}$ with 395-, 592- and 229-fold increases in the fluorescence intensity at $743 \mathrm{~nm}$ with $\mathrm{pH}$ decrease from 7.4 to 4.1 , respectively. The $\mathrm{pK}_{\text {cycl }}$ values of the probe $\mathbf{B}, \mathbf{C}$ and $\mathbf{D}$ related to the spirolactam ring opening are 4.6, 4.9 and 5.4, respectively, which indicates that the probes $\mathbf{B}, \mathbf{C}$ and $\mathbf{D}$ are more suitable for 
lysosome imaging application. However, further decrease of $\mathrm{pH}$ to strong acidic conditions triggers significant fluorescence decreases of fluorescent probe $\mathbf{A}$ because the protonation of the nitrogen atom of the fluorophore significantly reduces the electron donating ability of the nitrogen atom, and results in charge imbalance through the resonance structure (Scheme 3.3). The analysis of fluorescence intensity changes of fluorescent probe $\mathbf{A}$ as a function of $\mathrm{pH}$ by using the Henderson-Hasselbach-type mass action equation yielded almost same $\mathrm{pK}_{\mathrm{a}}$ value of 1.8. The similar $\mathrm{pH}$ effect on fluorescent probes $\mathbf{B}, \mathbf{C}$ and $\mathbf{D}$ were also observed with further decrease of $\mathrm{pH}$ to strong acidic conditions. The analysis of fluorescence intensity changes of fluorescent probes $\mathbf{B}, \mathbf{C}$ and $\mathbf{D}$ gave almost the same $\mathrm{pK}_{\mathrm{a}}$ value of 1.7 .
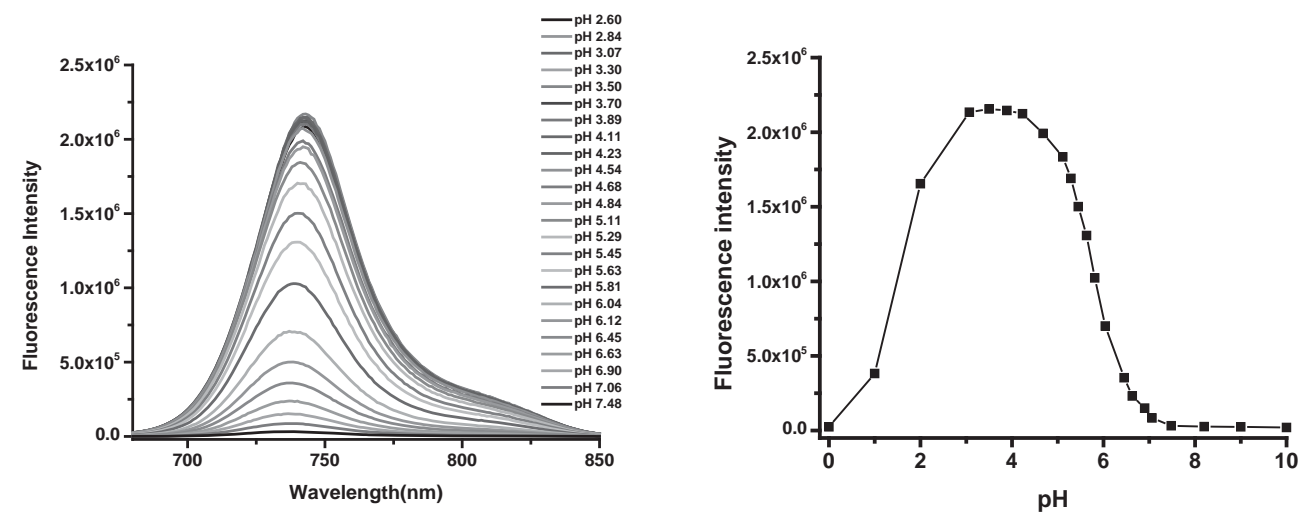

Figure 3.4. Fluorescent spectra of $5 \mu \mathrm{M}$ fluorescent probe $\mathbf{A}$ at different $\mathrm{pH}$ (left) and $\mathrm{pH}$ effect on fluorescence intensity of fluorescent probe $\mathbf{A}$ at $743 \mathrm{~nm}$ (right). 

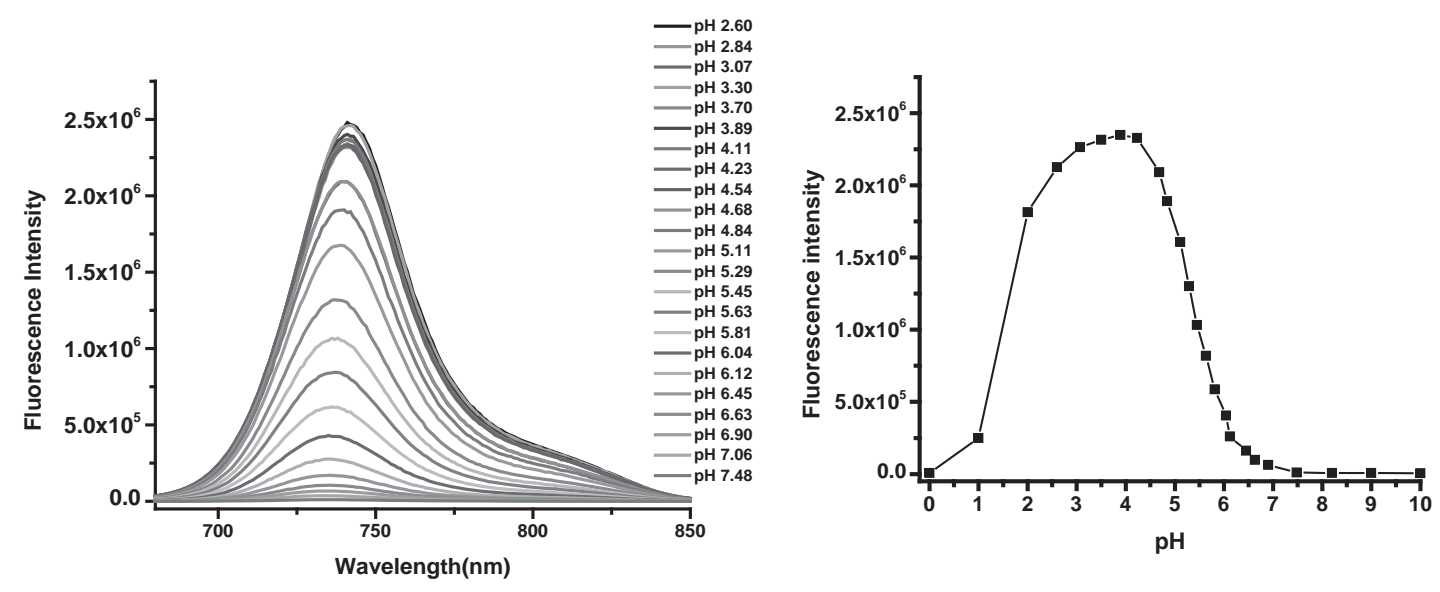

Figure 3.5. Fluorescent spectra of $5 \mu \mathrm{M}$ fluorescent probe $\mathbf{D}$ at different $\mathrm{pH}$ (left) and $\mathrm{pH}$ effect on fluorescence intensity of fluorescent probe $\mathbf{D}$ at $743 \mathrm{~nm}$ (right).

\subsubsection{The selectivity experiments of fluorescent probes to $\mathrm{pH}$ over metal ions.}

We investigated effect of metal ions on fluorescent response of fluorescent probes to $\mathrm{pH}$ by evaluating potential coordination of fluorescent probes with heavy, transition, and main group metal ions. Fluorescent probes A, B, C and $\mathbf{D}$ display no responses to $200 \mu \mathrm{M}$ alkali and alkaline-earth metal ions as such $\mathrm{Na}^{+}, \mathrm{K}^{+}, \mathrm{Ca}^{2+}$ and $\mathrm{Mg}^{2+}$, as well as some transitional metal ions $(200 \mu \mathrm{M})$ such as $\mathrm{Cu}^{2+}, \mathrm{Zn}^{2+}, \mathrm{Fe}^{3+}, \mathrm{Fe}^{2+}, \mathrm{Co}^{2+}, \mathrm{Ag}+, \mathrm{Ba}^{2+}, \mathrm{Cd}^{2+}, \mathrm{Pb}^{2+}, \mathrm{Ni}^{2+}$ and $\mathrm{Mn}^{2+}$ at $\mathrm{pH} 7.0$ and 4.1 (Figure 3.6, and Figures B.21-23), which indicates that the fluorescent probes display high selectivity to $\mathrm{pH}$ over these alkali, alkaline-earth metal ions, and transitional metal ions. 


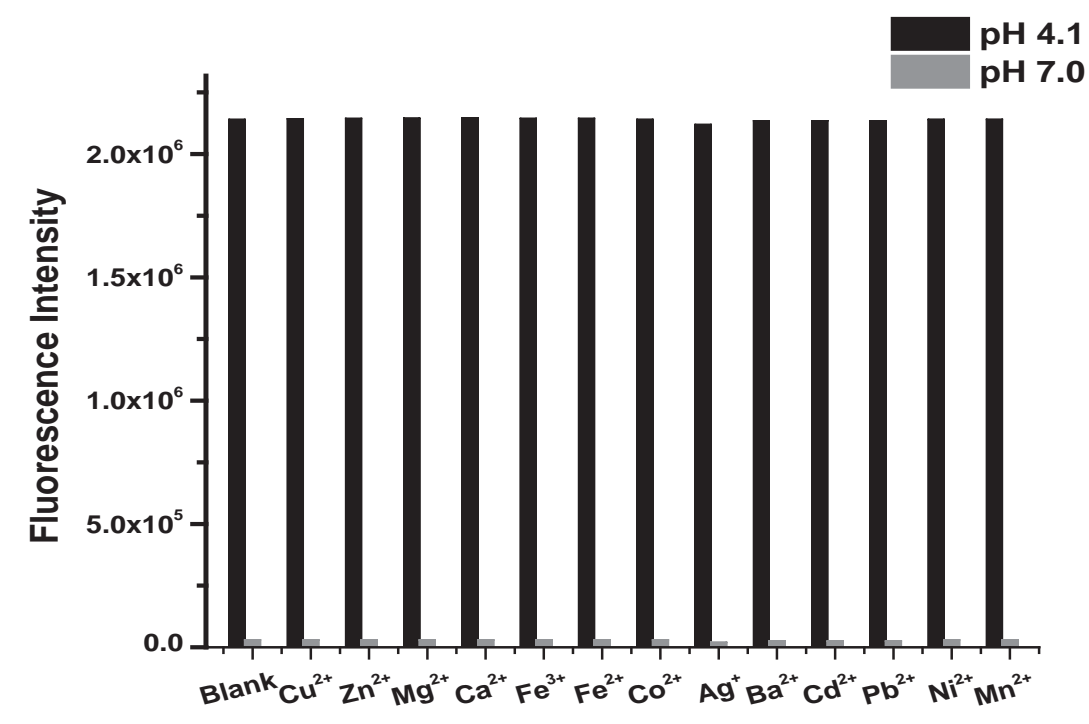

Figure 3.6. Fluorescent responses of $5 \mu \mathrm{M}$ fluorescent probe $\mathbf{A}$ to $\mathrm{pH}$ at 4.1 and 7.0 in the absence and presence of different metal ions $(200 \mu \mathrm{M})$, respectively.

\subsubsection{Live cell imaging of fluorescent probes}

All four fluorescent probes were used as near-infrared fluorescent probes in cultured cells and compared to commercial LysoSensor Green DND-189 to investigate if these probes could be used to target lysosomes/acidic organelles inside the cells as LysoSensor Green DND-189 is known to be retained specifically in acidic organelles. A series of experiments were performed: a breast cancer line (MDA-MB-231) and normal endothelial cell line (HUVEC) were loaded with fluorescent probes A, B, C and D and with LysoSensor Green DND-189 (Figures 3.7 and 3.8), respectively. All the probes except probe A showed a fluorescence signal inside the cell, specifically localized in lysosomes with maximum signal with probe $\mathbf{D}$ followed by probe $\mathbf{B}$ (Figures 3.7-9). The fluorescence signals of these probes were compared with the commercially available and well characterized lysosome specific probe, LysoSensor Green DND-189 (Lyso-green). To confirm the co-localization of these probes to lysosomes, we incubated both the cells with Lyso-green and these new probes. Images were captured for the same image field with different excitation and 
emission wavelengths of near-infrared fluorescent probes as compared to those of probe DND-189, which enables simultaneous visualization of both probes (near-infrared probes and DND-189) from the same intracellular compartment. When the images were overlaid they showed co-localization in lysosomes (Figures 3.7-9). The probe D was most fluorescent and showed a very high signal even at $5 \mu \mathrm{M}$ concentration and was clearly colocalized to lysosomes (Figure 3.9). The fluorescence intensity of the probe $\mathbf{D}$ is shown to match that of the probe DND-189 as the areas of low and high fluorescence of the probe match those of the probe DND-189, indicating the probe $\mathbf{D}$ can effectively distinguish between different $\mathrm{pH}$ values in the cell in a similar manner to the commercial probe DND189. Probe $\mathbf{B}$ showed a slightly weaker signal when compared to probe $\mathbf{D}$ (Figures 3.7-8). The close-up of co-localization of cells image clearly shows that the lysosomes were mostly localized as perinuclear clusters both in cancer and normal cell lines (Figure 3.9). These results are consistent with a previous report that showed lysosomes in serum-starved cells relocate towards the perinuclear position and forms clusters. ${ }^{27}$ In this study, the probes were incubated in serum-free media thus the lysosomes localize to perinuclear clusters. This data also confirms that the probes are localized only in lysosomes and are highly sensitive to $\mathrm{pH}$ environment. 


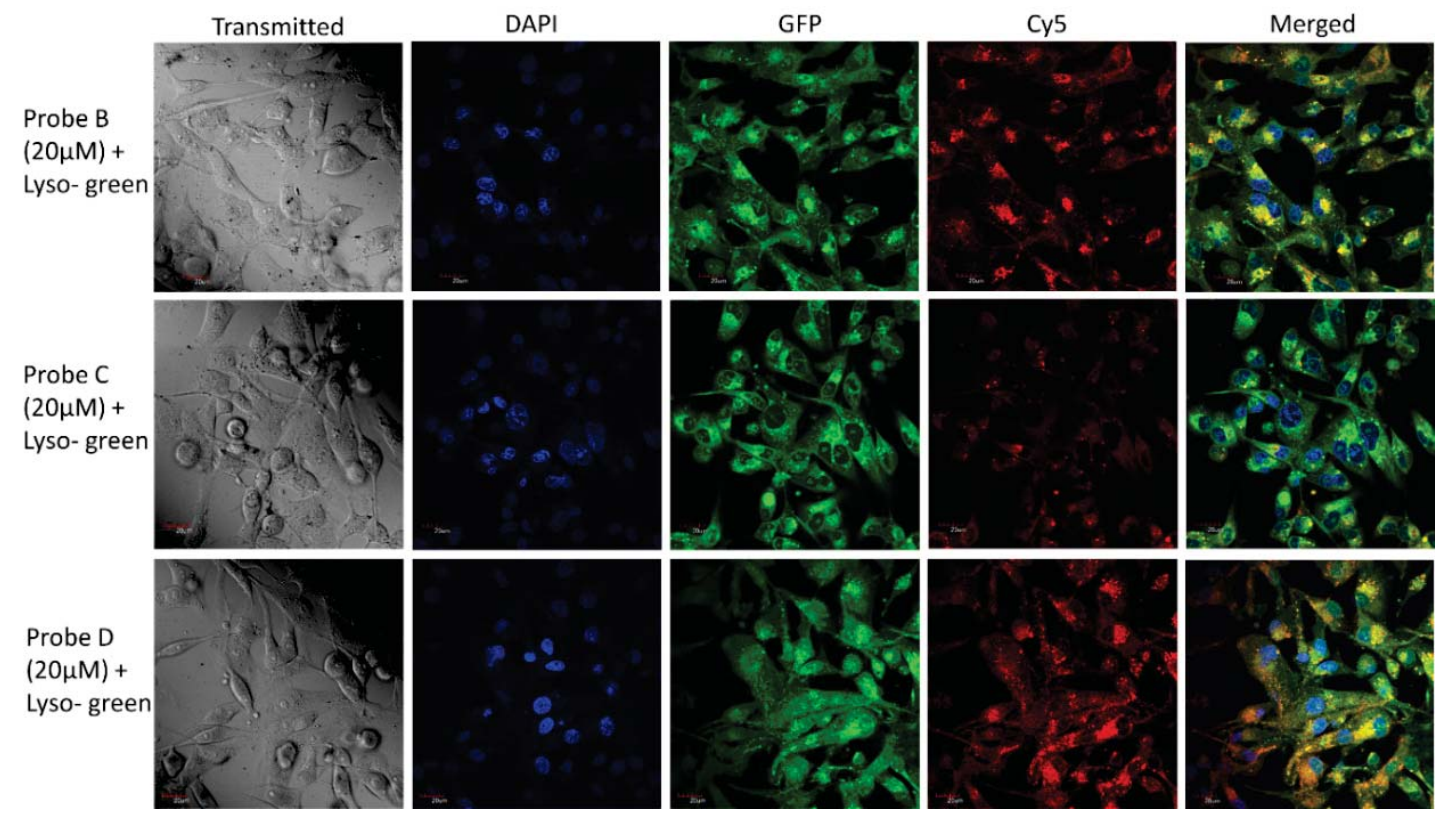

Figure 3.7. Fluorescence images of MDA-MB-231 cells incubated with fluorescent probes $\mathbf{B}, \mathbf{C}$, and D. Cells were incubated with $20 \mu \mathrm{M}$ of dyes $\mathbf{B}, \mathbf{C}$, and $\mathbf{D}$ for $2 \mathrm{~h}$ and imaged for co-localization in presence of LysoSensor Green, a lysosomal stain and Hoechest, a nuclear stain. The images were acquired using confocal fluorescence microscope at $60 \mathrm{X}$ magnification. 


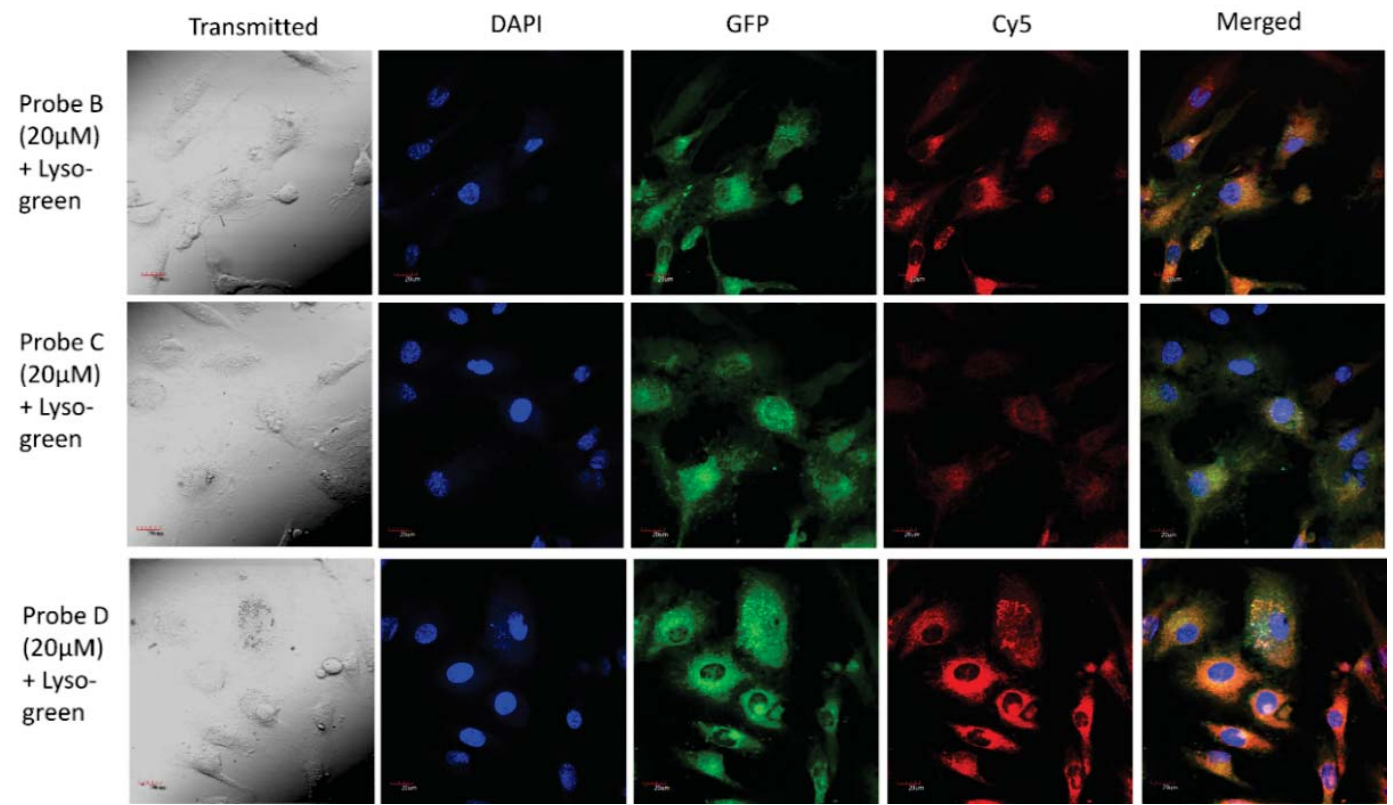

Figure 3.8. Fluorescence images of HUVEC-C cells incubated with fluorescent probes B, C, and D. Cells were incubated with $20 \mu \mathrm{M}$ of all three dyes for $2 \mathrm{~h}$ and imaged for colocalization with LysoSensor Green and Hoechest stains. The images were acquired using confocal fluorescence microscope at 60X magnification.

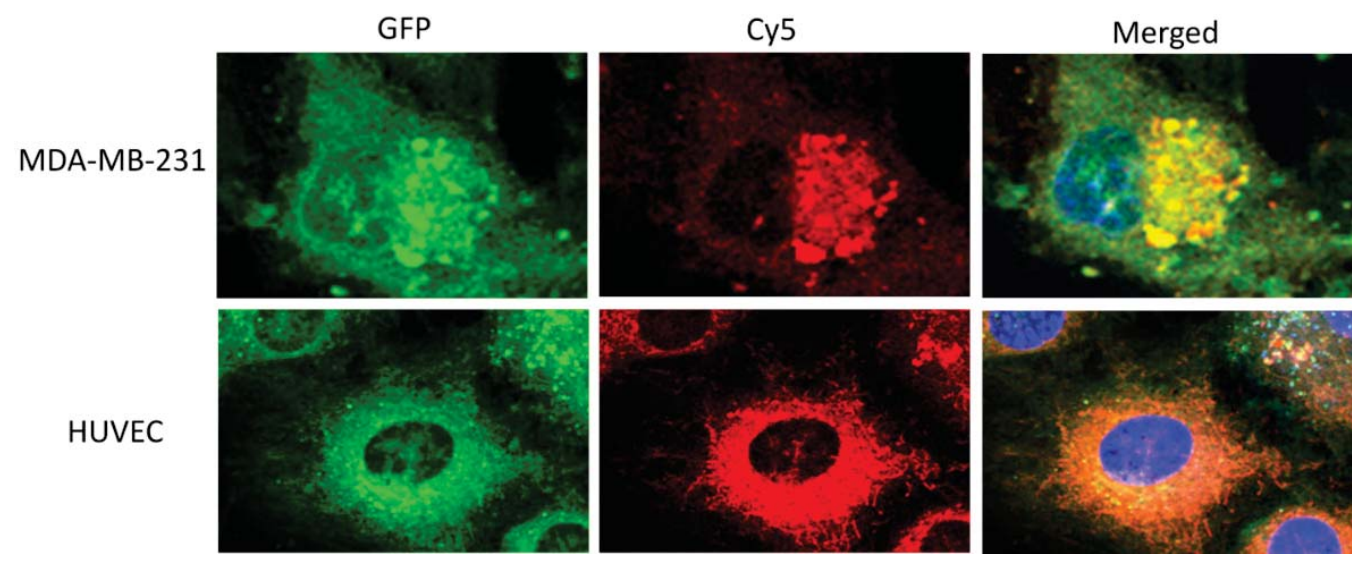

Figure 3.9. Enlarged images of MDA-MB-231 and HUVEC-C cells with fluorescent probe $\mathbf{D}$ showing co-localization of fluorescent probe $\mathbf{D}$ in lysosomes. The probe $\mathbf{D}$ shows much stronger signal in HUVEC cells compared to MDA-MB-231 cell. The lysosomes are mostly perinuclear in both cells due to serum-starvation for $2 \mathrm{~h}$. 


\subsection{Summary}

We have prepared four near-infrared fluorescent probes for $\mathrm{pH}$ (A-D). The response mechanism of the fluorescent probes to $\mathrm{pH}$ value relies on the structural changes between spirocylic and ring-opening forms of the near-infrared fluorophore. These probes are not fluorescent with a spriocylic form at neutral $\mathrm{pH}$ and highly fluorescent with a spirocycleopening at low $\mathrm{pH}$ value of $\sim 4.5$. Fluorescent probes have absorption and emission peaks at $718 \mathrm{~nm}$ and $743 \mathrm{~nm}$, respectively. The fluorescent probes $\mathbf{B}, \mathbf{C}$ and $\mathbf{D}$ are cell-permeable and are capable of selective and sensitive labeling of lysosomes, and may offer potential noninvasive monitoring of lysosomal $\mathrm{pH}$ changes during physiological and pathological processes.

\section{Supporting Information}

${ }^{1} \mathrm{H}$ and ${ }^{13} \mathrm{C}$ NMR, absorption and emission spectra of the fluorescent probes, absorption and fluorescent spectra of the fluorescent probes at different $\mathrm{pH}$ values, and fluorescent spectra of fluorescent probes at $\mathrm{pH} 7.0$ and 4.1 in the absence and presence of different metal ions, respectively. This material is available free of charge via the Internet at http://pubs.acs.org.

\section{AUTHOR INFORMATION}

*E-mail: hyliu@mtu.edu

*E-mail: tiwari@mtu.edu

*E-mail: luoft@gate.sinica.edu.tw

\section{Notes}

The authors declare no competing financial interest. 


\section{ACKNOWLEDGMENTS}

This work was supported partially by National Science Foundation (to H.Y. Liu), ALS Therapy Alliance (to A. Tiwari), and Pruett postdoctoral fellowship support for J. Janjanam (to A. Tiwari).

\section{References}

(1) Burleigh, S. C., van de Laar, T., Stroop, C. J. M., van Grunsven, W. M. J., O'Donoghue, N., Rudd, P. M., and Davey, G. P. (2011) Synergizing metabolic flux analysis and nucleotide sugar metabolism to understand the control of glycosylation of recombinant protein in $\mathrm{CHO}$ cells. BMC Biotechnol. 11.

(2) Humez, S., Monet, M., van Coppenolle, F., Delcourt, P., and Prevarskaya, N. (2004) The role of intracellular $\mathrm{pH}$ in cell growth arrest induced by ATP. American Journal of Physiology-Cell Physiology 287, C1733-C1746.

(3) Edinger, A. L., and Thompson, C. B. (2004) Death by design: apoptosis, necrosis and autophagy. Curr. Opin. Cell Biol. 16, 663-669.

(4) Johansson, A. C., Appelqvist, H., Nilsson, C., Kagedal, K., Roberg, K., and Ollinger, K. (2010) Regulation of apoptosis-associated lysosomal membrane permeabilization. Apoptosis 15, 527-540.

(5) Trombetta, E. S., Ebersold, M., Garrett, W., Pypaert, M., and Mellman, I. (2003) Activation of lysosomal function during dendritic cell maturation. Science 299, 1400-1403.

(6) Urano, Y., Asanuma, D., Hama, Y., Koyama, Y., Barrett, T., Kamiya, M., Nagano, T., Watanabe, T., Hasegawa, A., Choyke, P. L., and Kobayashi, H. (2009) Selective molecular imaging of viable cancer cells with $\mathrm{pH}$-activatable fluorescence probes. Nat. Med. 15, 104-109.

(7) Turk, B., and Turk, V. (2009) Lysosomes as "Suicide Bags" in Cell Death: Myth or Reality? J. Biol. Chem. 284, 21783-21787. 
(8) Stinchcombe, J., Bossi, G., and Griffiths, G. M. (2004) Linking albinism and immunity: The secrets of secretory lysosomes. Science 305, 55-59.

(9) Blott, E. J., and Griffiths, G. M. (2002) Secretory lysosomes. Nature Reviews Molecular Cell Biology 3, 122-131.

(10) He, S., Mason, R. P., Hunjan, S., Mehta, V. D., Arora, V., Katipally, R., Kulkarni, P. V., and Antich, P. P. (1998) Development of novel F-19 NMR pH indicators: Synthesis and evaluation of a series of fluorinated vitamin B-6 Analogues. Biorg. Med. Chem. 6, 1631-1639.

(11) Diwu, Z. J., Chen, C. S., Zhang, C. L., Klaubert, D. H., and Haugland, R. P. (1999) A novel acidotropic $\mathrm{pH}$ indicator and its potential application in labeling acidic organelles of live cells. Chem. Biol. 6, 411-418.

(12) Lv, H. S., Liu, J., Zhao, J., Zhao, B. X., and Miao, J. Y. (2013) Highly selective and sensitive $\mathrm{pH}-$ responsive fluorescent probe in living Hela and HUVEC cells. Sens. Actuator B-Chem. 177, 956-963.

(13) Zhu, H., Fan, J. L., Xu, Q. L., Li, H. L., Wang, J. Y., Gao, P., and Peng, X. J. (2012) Imaging of lysosomal $\mathrm{pH}$ changes with a fluorescent sensor containing a novel lysosome-locating group. Chem. Commun. 48, 11766-11768.

(14) Li, Z., Song, Y. L., Yang, Y. H., Yang, L., Huang, X. H., Han, J. H., and Han, S. F. (2012) Rhodamine-deoxylactam functionalized poly styrene-alter-(maleic acid) $\mathrm{s}$ as lysosome activatable probes for intraoperative detection of tumors. Chem. Sci. 3, 2941-2948.

(15) Ying, L. Q., and Branchaud, B. P. (2011) Selective labeling and monitoring pH changes of lysosomes in living cells with fluorogenic pH sensors. Bioorg. Med. Chem. Lett. 21, 3546-3549.

(16) Smith, D. G., McMahon, B. K., Pal, R., and Parker, D. (2012) Live cell imaging of lysosomal $\mathrm{pH}$ changes with $\mathrm{pH}$ responsive ratiometric lanthanide probes. Chem. Commun. 48, 8520-8522.

(17) Ma, L. J., Cao, W. G., Liu, J. L., Deng, D. Y., Wu, Y. Q., Yan, Y. H., and Yang, L. T. (2012) A highly selective and sensitive fluorescence dual-responsive $\mathrm{pH}$ probe in water. Sens. Actuator B-Chem. 169, 243-247. 
(18) Galindo, F., Burguete, M. I., Vigara, L., Luis, S. V., Kabir, N., Gavrilovic, J., and Russell, D. A. (2005) Synthetic macrocyclic peptidomimetics as tunable $\mathrm{pH}$ probes for the fluorescence imaging of acidic organelles in live cells. Angew. Chem. Int. Ed. 44, 6504-6508.

(19) DePedro, H. M., and Urayama, P. (2009) Using LysoSensor Yellow/Blue DND160 to sense acidic pH under high hydrostatic pressures. Anal. Biochem. 384, 359361.

(20) Hasegawa, T., Kondo, Y., Koizumi, Y., Sugiyama, T., Takeda, A., Ito, S., and Hamada, F. (2009) A highly sensitive probe detecting low pH area of HeLa cells based on rhodamine B modified beta-cyclodextrins. Biorg. Med. Chem. 17, 60156019.

(21) Lin, H. J., Herman, P., Kang, J. S., and Lakowicz, J. R. (2001) Fluorescence lifetime characterization of novel low-pH probes. Anal. Biochem. 294, 118-125.

(22) Fan, J. L., Lin, C. Y., Li, H. L., Zhan, P., Wang, J. Y., Cui, S., Hu, M. M., Cheng, G. H., and Peng, X. J. (2013) A ratiometric lysosomal pH chemosensor based on fluorescence resonance energy transfer. Dyes Pigment. 99, 620-626.

(23) Han, J. Y., and Burgess, K. (2010) Fluorescent Indicators for Intracellular pH. Chem. Rev. 110, 2709-2728.

(24) Hu, Z. Q., Li, M., Liu, M. D., Zhuang, W. M., and Li, G. K. (2013) A highly sensitive fluorescent acidic $\mathrm{pH}$ probe based on rhodamine B diethyl-2aminobutenedioate conjugate and its application in living cells. Dyes Pigment. 96, $71-75$.

(25) Zhu, S. L., Zhang, J. T., Janjanam, J., Bi, J. H., Vegesna, G., Tiwari, A., Luo, F. T., Wei, J. J., and Liu, H. Y. (2013) Highly water-soluble, near-infrared emissive BODIPY polymeric dye bearing RGD peptide residues for cancer imaging. Anal. Chim. Acta 758, 138-144.

(26) Yuan, L., Lin, W. Y., Yang, Y. T., and Chen, H. (2012) A Unique Class of NearInfrared Functional Fluorescent Dyes with Carboxylic-Acid-Modulated Fluorescence ON/OFF Switching: Rational Design, Synthesis, Optical Properties, 
Theoretical Calculations, and Applications for Fluorescence Imaging in Living Animals. J. Am. Chem. Soc. 134, 1200-1211.

(27) Korolchuk, V. I., Saiki, S., Lichtenberg, M., Siddiqi, F. H., Roberts, E. A., Imarisio, S., Jahreiss, L., Sarkar, S., Futter, M., Menzies, F. M., O'Kane, C. J., Deretic, V., and Rubinsztein, D. C. (2011) Lysosomal positioning coordinates cellular nutrient responses. Nat. Cell Biol. 13, 453-U242. 


\section{Chapter 4 : Synthesis of a Hybrid Dye, Tested its Reactivity for Fluorescent Probe Design ${ }^{3}$}

Giri Vegesna, ${ }^{a}$, Fen-Tair Luo ${ }^{b^{*}}$, and Haiying Liu ${ }^{a^{*}}$

aDepartment of Chemistry, Michigan Technological University, Houghton, MI 49931

${ }^{b}$ Institute of Chemistry, Academia Sinica, Taipei, Taiwan 11529, Republic of China

${ }^{3}$ This chapter may be submitted to a journal for possible publication in the near future. 


\subsection{Abstract}

A facile and scalable synthesis of new functionalizable fluorescent hybrid dye $\mathbf{A}$ has been designed synthesized and characterized. This dye has absorption and emission wavelength at 610 and $640 \mathrm{~nm}$ respectively, fluorescent quantum yield $22.3 \%$ in ethanol and molar absorption coefficient $1.66 \times 10^{4} \mathrm{M}^{-1} \cdot \mathrm{cm}^{-1}$. This new hybrid dye has reactive chlorine atom which can be functionalized to prepare imaging probes and also there is also scope for introducing water soluble groups. Three new compounds B, C, D were synthesized by reacting with primary and secondary amine to test the reactivity of chlorine atom of the hybrid dye. This concludes that this hybrid dye can be used to prepare fluorescent probes using different receptors.

\subsection{Introduction}

To study the biomolecules in live cells, fluorescent imaging is one of the most powerful techniques. Fluorescent dye are the essential molecular tools for the bioimaging technique. Although a large number of fluorescent probes are reported in literature till now, most of them have absorption and emission at 450-600 $\mathrm{nm}$ range only. Very less number of fluorescent probes with emission at $600-800 \mathrm{~nm}$ range are reported so far. In addition, the NIR probes have advantages over the visible range probes such as, deep tissue penetration, low auto fluorescence and minimum photo damage. So there is a lot of demand for NIR probes that exhibit good photostability for bio imaging applications.

Cyanine dyes are one class of NIR fluorescent molecules that are most widely used in bio imaging probe design. To synthesize a Cyanine based turn-on probe, fluorescence resonance energy transfer (FRET) approach is one strategy. For the FRET based cyanine probe design, an additional fluorescent dye is needed which makes the system more complex. Due to the relatively high energy levels of highest occupied molecular orbitals (HOMO) of cyanine dyes, it also quite difficult to synthesize photo induced electron (PET) based turn-on fluorescent cyanine probes. So there is lot of demand for easily Tunable NIR fluorescent dyes that can be have applications in bioimaging. 


\subsection{Experimental Section}

\subsubsection{Instrumentation}

${ }^{1} \mathrm{H}$ NMR and ${ }^{13} \mathrm{C}$ NMR spectra were obtained by using a $400 \mathrm{MHz}$ NMR spectrophotometer instrument. ${ }^{1} \mathrm{H}$ and ${ }^{13} \mathrm{C}$ NMR spectra were recorded in $\mathrm{CDCl}_{3}$, chemical shifts $(\delta)$ are given in ppm relative to solvent peaks $\left({ }^{1} \mathrm{H}: \delta 7.26 ;{ }^{13} \mathrm{C}: \delta 77.3\right)$ as internal standard. HRMS were measured with fast atom bombardment (FAB) ionization or matrixassisted laser desorption ionization (MALDI) using a double focusing magnetic or a matrix assisted laser desorption-time of flight mass spectrometer. Infrared (IR) samples were prepared as $\mathrm{KBr}$ pellets, and spectra were obtained in the $400-4000 \mathrm{~cm}^{-1}$ range using a Bruker Tensor-27 FTIR spectrometer. Absorption spectra were taken on a Perkin Elmer Lambda 35 UV/VIS spectrometer. Fluorescence spectra were recorded on a on a Jobin Yvon Fluoromax-4 spectrofluorometer. Fluorescence quantum yield of fluorescent dyes $\mathbf{A}, \mathbf{B}, \mathbf{C}$, and $\mathbf{D}$ in ethanol was measured by using standard 1-cm path-length fluorescence quartz cuvette at room temperature. All samples were scanned with increments of $1 \mathrm{~nm}$. Each of the spectrum for absorbance and fluorescence was measured under room temperature. The new dye was dissolved in $3 \mathrm{ml} \mathrm{EtOH} / \mathrm{Buffer}(\mathrm{v} / \mathrm{v} 2: 3)$ and got $5 \mu \mathrm{M}$ solution. The excitation wavelength at $550 \mathrm{~nm}$ was used to excite all fluorescent dye A and excitation wavelength at $500 \mathrm{~nm}$ was used to excite all fluorescent dyes $\mathbf{B}, \mathbf{C}$, and $\mathbf{D}$.

\subsubsection{Materials}

Unless otherwise indicated, all reagents and solvents were obtained from commercial suppliers and used without further purification. Air- and moisture-sensitive reactions were conducted in oven-dried glassware using a standard Schlenk line or drybox techniques under an inert atmosphere of dry nitrogen. 

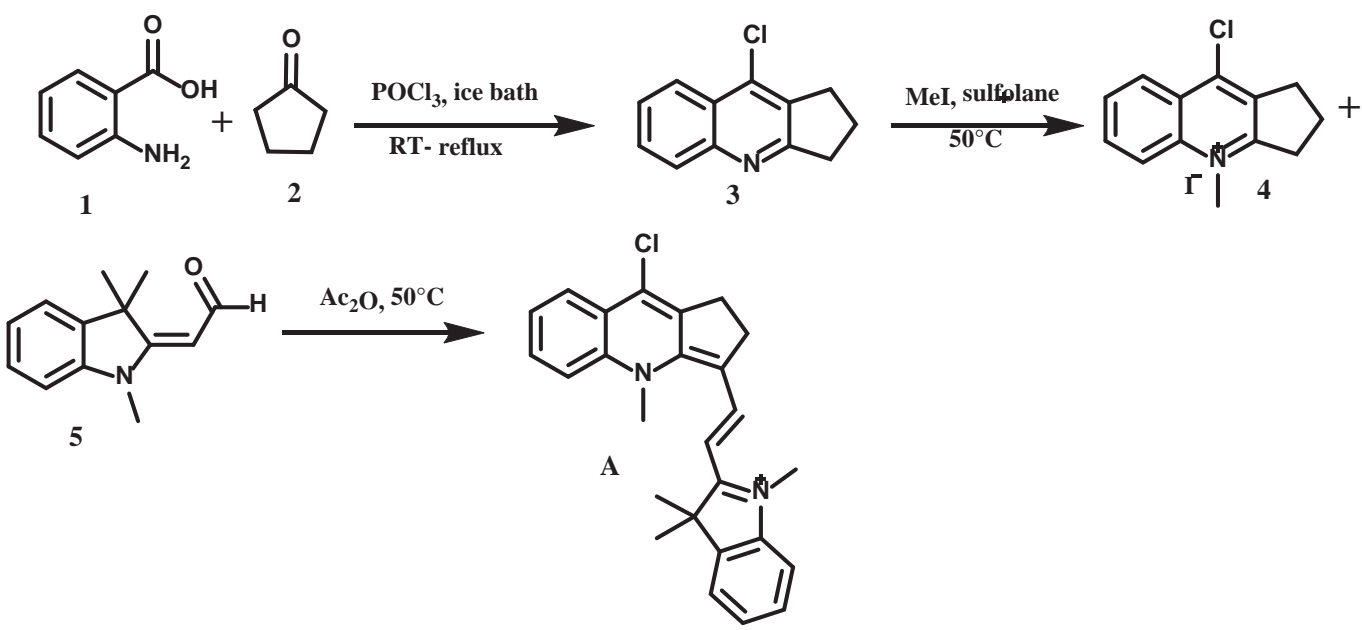

Scheme 4.1. Synthetic route to hybrid fluorescent dye A

Synthesis of compound 3: The mixture of compound 1 ( $4.0 \mathrm{~g}, 30.0 \mathrm{mmol})$ cyclopentanone $2(2.65 \mathrm{ml}, 30.0 \mathrm{mmol})$ at ice bath, $\mathrm{POCl}_{3}(25 \mathrm{ml})$ was added slowly. The resultant mixture was heated to reflux for $2 \mathrm{hrs}$. Then cool the reaction mass to room temperature and concentrated under vacuum. The residue was diluted with EtOAc (70 ml), neutralized with potassium carbonate. Compound was washed with water $(2 \times 100 \mathrm{ml})$ and brine $(2 \times 100 \mathrm{ml})$ solution. Organic layer was dried over sodium sulfate and concentrated under reduced pressure to yield a pale brown solid. The crude solid was recrystallized from acetone to give the compound 3 (5.3 g, 85\%). ${ }^{1} \mathrm{H}$ NMR (400 MHz, $\left.\mathrm{CDCl}_{3}\right): \delta 8.14-8.12$ (d, $J=8.0$ $\mathrm{Hz}, 1 \mathrm{H}), 8.02-7.99$ (d, $J=12.0 \mathrm{~Hz}, 1 \mathrm{H}), 7.67-7.63$ (t, $J=8.0 \mathrm{~Hz}, 1 \mathrm{H}), 7.55-7.51$ (t, $J=8.0$ $\mathrm{Hz}, 1 \mathrm{H}$ ), 3.23-3.19 (t, $J=8.0 \mathrm{~Hz}, 2 \mathrm{H}), 3.16-3.12$ (t, $J=8.0 \mathrm{~Hz}, 2 \mathrm{H}), 2.26-2.18$ (p, $J=8.0$ $\mathrm{Hz}, 2 \mathrm{H}) .{ }^{13} \mathrm{C} \mathrm{NMR}\left(100 \mathrm{MHz}, \mathrm{CDCl}_{3}\right): \delta 167.71,148.69,137.80,134.23,129.41,129.05$, 126.63, 125.50, 123.73, 35.71, 30.61, 22.88. HRMS (ESI) calcd for $\mathrm{C}_{12} \mathrm{H}_{10} \mathrm{ClN}[\mathrm{M}]^{+}$, 203.0498; found, 203.0502 .

Synthesis of compound 4: To the solution of compound $3(2.0 \mathrm{~g}, 10.0 \mathrm{mmol})$ in tetramethylene sulfone $(20 \mathrm{ml})$ at room temperature was treated with methyl iodide (4.0 $\mathrm{ml}, 64 \mathrm{mmol})$. The resulting reaction mass was heated to reflux for $12 \mathrm{hrs}$. After cooling to room temperature, the mixture was filtered, washed with dry ether to remove the organic impurities. The solid obtained was dried under vacuum to yield a yellowish green solid $\mathbf{4}$ (3.75 g, 44\%). ${ }^{1} \mathrm{H}$ NMR (400 MHz, DMSO- $\left.d_{6}\right): \delta 8.55-8.53(\mathrm{~d}, J=8.0 \mathrm{~Hz}, 1 \mathrm{H}), 8.49-8.47$ 
(d, $J=8.0 \mathrm{~Hz}, 1 \mathrm{H}), 8.24-8.20(\mathrm{~m}, 1 \mathrm{H}), 8.08-8.04(\mathrm{t}, J=8.0 \mathrm{~Hz}, 1 \mathrm{H}), 4.40(\mathrm{~s}, 3 \mathrm{H}), 3.68-$ $3.64(\mathrm{t}, J=8.0 \mathrm{~Hz}, 2 \mathrm{H}), 3.32-3.28(\mathrm{t}, J=8.0 \mathrm{~Hz}, 2 \mathrm{H}), 2.36-2.28(\mathrm{p}, J=8.0 \mathrm{~Hz}, 2 \mathrm{H}) .{ }^{13} \mathrm{C}$ NMR (100 MHz, DMSO- $\left.d_{6}\right): \delta 169.17,145.34,139.27,138.65,135.12,130.93,126.01$, $120.19,94.43,41.75,36.02,31.77,21.96$.

Synthesis of compound A: Compound $4(1.0 \mathrm{~g}, 2.65 \mathrm{mmol})$ and fisher aldehyde $5(0.53 \mathrm{~g}$, $2.65 \mathrm{mmol})$ were dissolved in acetic anhydride $(20 \mathrm{~mL})$ and the resulting reaction mixture was heated to $50^{\circ} \mathrm{C}$ for $30 \mathrm{Min}$. After quenching the reaction with water, acetic anhydride was distilled off. The crude Compound was dissolved in dichloromathnae $(40 \mathrm{~mL})$ and washed sequentially with aqueous sodium bi carbonate $(2 \times 60 \mathrm{~mL})$, water $(2 \times 60 \mathrm{~mL})$ and brine solution $(1 \times 60 \mathrm{~mL})$. Dry the organic layer with sodium sulfate and distil off the solvent under reduced pressure. The crude Compound was purified by column chromatography with DCM/EtOH (100:1 to 20:1) to yield a greenish solid (1.2 g, 81\%). ${ }^{1} \mathrm{H}$ NMR (400 MHz, CDCl3): $\delta$ 8.12-8.06 (m, 2H), 7.98-7.94 (m, 1H), 7.80-7.76 (m, 1H), 7.52-7.48 (m, 1H), 7.30-7.24 (m, 2H), 7.12-7.08 (m, 1H), 7.04-7.00 (m, 1H), 5.72-5.70 (m, $1 \mathrm{H}), 4.4(\mathrm{~s}, 3 \mathrm{H}), 3.62(\mathrm{~S}, 4 \mathrm{H}), 1.70(\mathrm{~s}, 6 \mathrm{H})$.
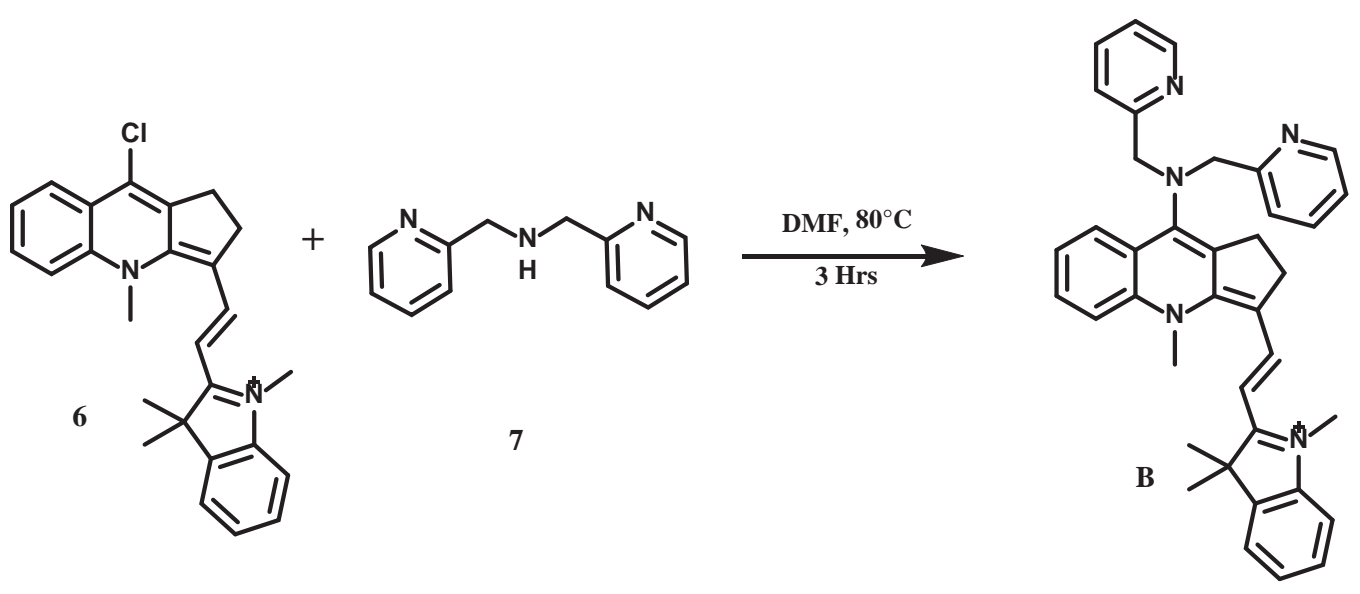

Scheme 4.2. Synthetic route to Compound B

Synthesis of compound B: Compound 6 ( $0.2 \mathrm{~g}, 0.49 \mathrm{mmol})$ and compound 7 (0.39 g,1.96 mmol) were dissolved in dry DMF (30 Ml) under nitrogen atmosphere. The reaction mixture was heated to $80^{\circ} \mathrm{C}$ for $3 \mathrm{hrs}$. Distill off the DMF under high vacuum and the crude compound was purified by column chromatography using DCM/EtOH (50/1 to 10/1) to 
yield a dark blue solid (0.08 g, 30\%). ${ }^{1} \mathrm{H}$ NMR (400 MHz, $\left.\mathrm{CDCl}_{3}\right): \delta 8.52-8.51(\mathrm{~d}, J=4.0$ $\mathrm{Hz}, 2 \mathrm{H}), 8.27-8.24$ (d, $J=12.0 \mathrm{~Hz}, 1 \mathrm{H}), 8.11-8.08$ (d, $J=12.0 \mathrm{~Hz}, 1 \mathrm{H}), 7.87-7.53(\mathrm{~m}, 5 \mathrm{H})$, 7.33-7.31 (d, $J=8.0 \mathrm{~Hz}, 2 \mathrm{H}), 7.27-7.17(\mathrm{~m}, 4 \mathrm{H}), 7.03-6.99(\mathrm{t}, J=8.0 \mathrm{~Hz}, 1 \mathrm{H}), 6.88-6.86$ $(\mathrm{d}, J=8.0 \mathrm{~Hz}, 1 \mathrm{H}), 5.49-5.46(\mathrm{~d}, J=12.0 \mathrm{~Hz}, 1 \mathrm{H}), 4.65(\mathrm{~s}, 4 \mathrm{H}), 4.43(\mathrm{~s}, 3 \mathrm{H}), 3.36(\mathrm{~s}, 3 \mathrm{H})$, 3.14-2.75 (m, 4H), 1.64(s, 6H). ${ }^{13} \mathrm{C}$ NMR (100 MHz, $\left.\mathrm{CDCl}_{3}\right): \delta 168.11,159.43,156.90$, $153.59,149.59,143.61,142.08,139.81,138.38,137.73,133.96,132.88,128.47,126.80$, $126.20,124.43,123.57,123.30,123.11,122.21,119.07,108.65,96.42,58.31,47.73$, 43.03, 30.65, 28.70, 27.70. HRMS (ESI) calcd for $\mathrm{C}_{38} \mathrm{H}_{38} \mathrm{~N}_{5}[\mathrm{M}]^{+}, 564.3127$; found, 564.3142 .

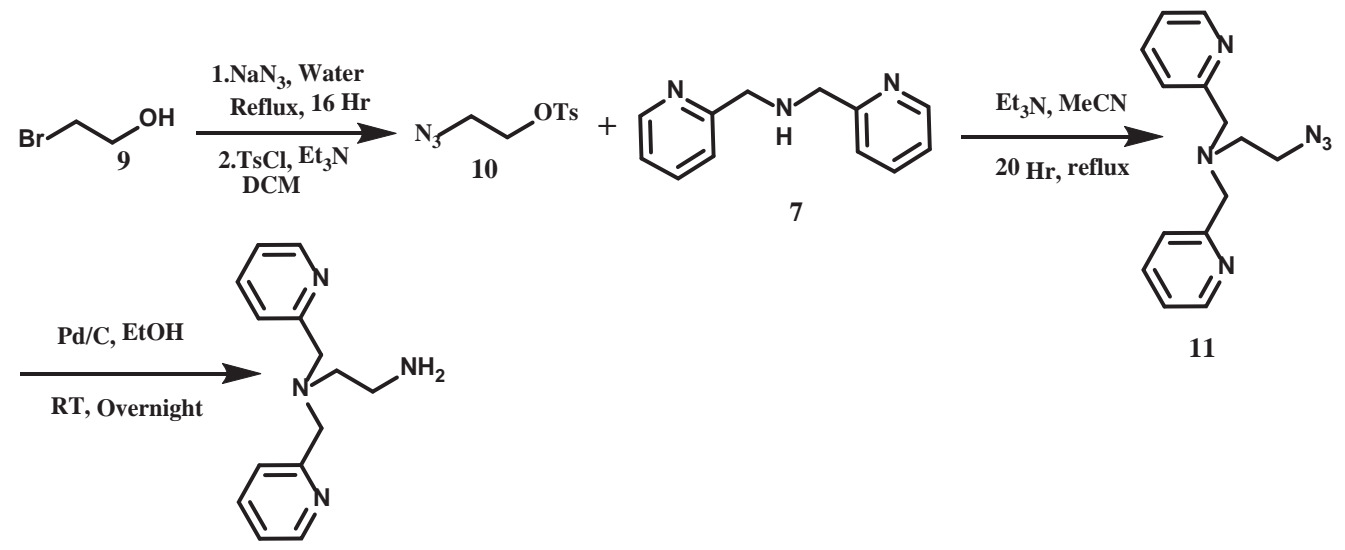

12

Scheme 4.3. Synthetic route to Compound 12

Synthesis of compound 10: In a round bottom flask, Bromoetahnol 9 (10 g, $80.02 \mathrm{mmol})$, water $(100 \mathrm{~mL})$ and Sodium azide $(6.2 \mathrm{~g}, 96.03 \mathrm{mmol})$ were added. This resulting reaction mixture was stirred at reflux overnight. Cool the solution, Extract the Azide compound into dichloromethane $(2 \times 100 \mathrm{~mL})$. The combined organic layers were washed with water $(2 \times 100 \mathrm{~mL})$, dried with anhydrous sodium sulphate and this azide in the dichloromethane was used as such for next step. To the azide compound $(6.8 \mathrm{~g}, 79.06 \mathrm{mmol})$ in round bottom flask, Triethylamine $(13.2 \mathrm{~mL}, 94.88 \mathrm{mmol})$ and P-toulenesulfonyl chloride $(18.08 \mathrm{~g}$, $94.88 \mathrm{mmol}$ ) was added. This mixture was stirred at room temperature for $6 \mathrm{hr}$. Reaction was quenched with water and the organic layer was washed with $1 \mathrm{~N} \mathrm{NaOH}(2 \times 200 \mathrm{~mL})$ 
and then with brine solution $(2 \times 200 \mathrm{~mL})$. Organic layer was dried with anhydrous sodium sulphate, solvent was evoparated under reduces pressure. The crude compound was purified by flash column chromatography with EtoAc/Hexane (20:80) to yield clear oil (17 g, 89\%). ${ }^{1} \mathrm{H}$ NMR (400 MHz, $\left.\mathrm{CDCl}_{3}\right): \delta 7.77-7.75(\mathrm{~d}, J=16.0 \mathrm{~Hz}, 2 \mathrm{H}), 7.33-7.31$ (d, $J=$ $8.0 \mathrm{~Hz}, 2 \mathrm{H}), 4.12-4.09$ (t, $J=6.0 \mathrm{~Hz}, 2 \mathrm{H}), 3.45-3.42(\mathrm{t}, J=6.0 \mathrm{~Hz}, 2 \mathrm{H}), 2.40(\mathrm{~s}, 3 \mathrm{H}) .{ }^{13} \mathrm{C}$ NMR (100 MHz, $\left.\mathrm{CDCl}_{3}\right): \delta$ 145.52, 132.69, 130.22, 128.11, 68.46, 49.76, 21.82

Synthesis of compound 11: In a round bottom flask, compund $\mathbf{1 0}(1 \mathrm{~g}, 4.14 \mathrm{mmol})$ and Di(2-picolyl) amine $7(0.74 \mathrm{~mL}, 4.14 \mathrm{mmol})$ were dissolved in acetonitrile $(20 \mathrm{~mL})$ under nitrogen atmosphere. To this solution triethylamine $(0.7 \mathrm{~mL}, 4.96 \mathrm{mmol})$ was added, the resulting reaction mixture was stirred under reflux overnight. Solvent was removed in vacuum, the residue was dissolved in dichloromethane $(30 \mathrm{~mL})$ and wash the organic layer sequentially with aqu. $\mathrm{K}_{2} \mathrm{CO}_{3}(2 \times 30 \mathrm{~mL})$ and brine solution $(2 \times 30 \mathrm{~mL})$. Organic layer was dried with anhydrous sodium sulphate and solvent was evaporated under reduced pressure. The crude compound was purified by column chromatography using Hexane/DCM/EtOH (4:5:1) to yield a brownish oil (0.9 g, 69\%). ${ }^{1} \mathrm{H}$ NMR (400 MHz, $\left.\mathrm{CDCl}_{3}\right): \delta 8.45-8.44(\mathrm{~m}$, 2H), 7.60-7.56 (m, 2H), 7.47-7.45 (d, $J=8.0 \mathrm{~Hz}, 2 \mathrm{H}), 7.09-7.06(\mathrm{~m}, 2 \mathrm{H}), 3.80(\mathrm{~s}, 4 \mathrm{H})$, 3.26-3.23 (t, $J=6.0 \mathrm{~Hz}, 2 \mathrm{H}), 2.77-2.74(\mathrm{t}, J=6.0 \mathrm{~Hz}, 2 \mathrm{H}) .{ }^{13} \mathrm{C} \mathrm{NMR}\left(100 \mathrm{MHz}, \mathrm{CDCl}_{3}\right)$ : $\delta$ 159.21, 149.19, 136.67, 123.16, 122.30, 60.72, 53.53, 49.24. HRMS (ESI) calcd for $\mathrm{C}_{14} \mathrm{H}_{17} \mathrm{~N}_{6}[\mathrm{M}+\mathrm{H}]^{+}, 269.1515$; found, 269.1522

Synthesis of compound 12: To the stirred solution of compound $\mathbf{1 1}(0.7 \mathrm{~g}, 2.61 \mathrm{mmol})$ in ethanol (20 mL), 10\% Pd/C (0.07 g, $10 \mathrm{wt} \%)$ was added. The reaction mixture was stirred under hydrogen atmosphere overnight. Reaction mass was filtered through Celite pad to get clear solution. Ethanol was then removed in vacuum to yield colourless oil ( $0.6 \mathrm{~g}$, 95\%) and this compound was used as such in the next step without purification. ${ }^{1} \mathrm{H}$ NMR (400 MHz, $\mathrm{CDCl}_{3}$ ): $\delta$ 8.47-8.45 (m, 2H), 7.60-7.55 (m, 2H), 7.44-7.42 (d, $\left.J=8.0 \mathrm{~Hz}, 2 \mathrm{H}\right)$, 7.10-7.04 (m, 2H), 3.78 (s, 4H), 2.74-2.71 (t, $J=12.0 \mathrm{~Hz}, 2 \mathrm{H}), 2.61-2.58$ (t, $J=12.0 \mathrm{~Hz}$, $2 \mathrm{H}), 1.43\left(\mathrm{~s}, 2 \mathrm{H}, \mathrm{NH}_{2}\right) .{ }^{13} \mathrm{C} \mathrm{NMR}\left(100 \mathrm{MHz}, \mathrm{CDCl}_{3}\right): \delta 159.83,149.24,136.53,123.12$, 122.14, 60.90, 57.65, 39.85. HRMS (ESI) calcd for $\mathrm{C}_{14} \mathrm{H}_{18} \mathrm{~N}_{4} \mathrm{Na}[\mathrm{M}+\mathrm{Na}]^{+}, 265.1429$; found, 265.1435 

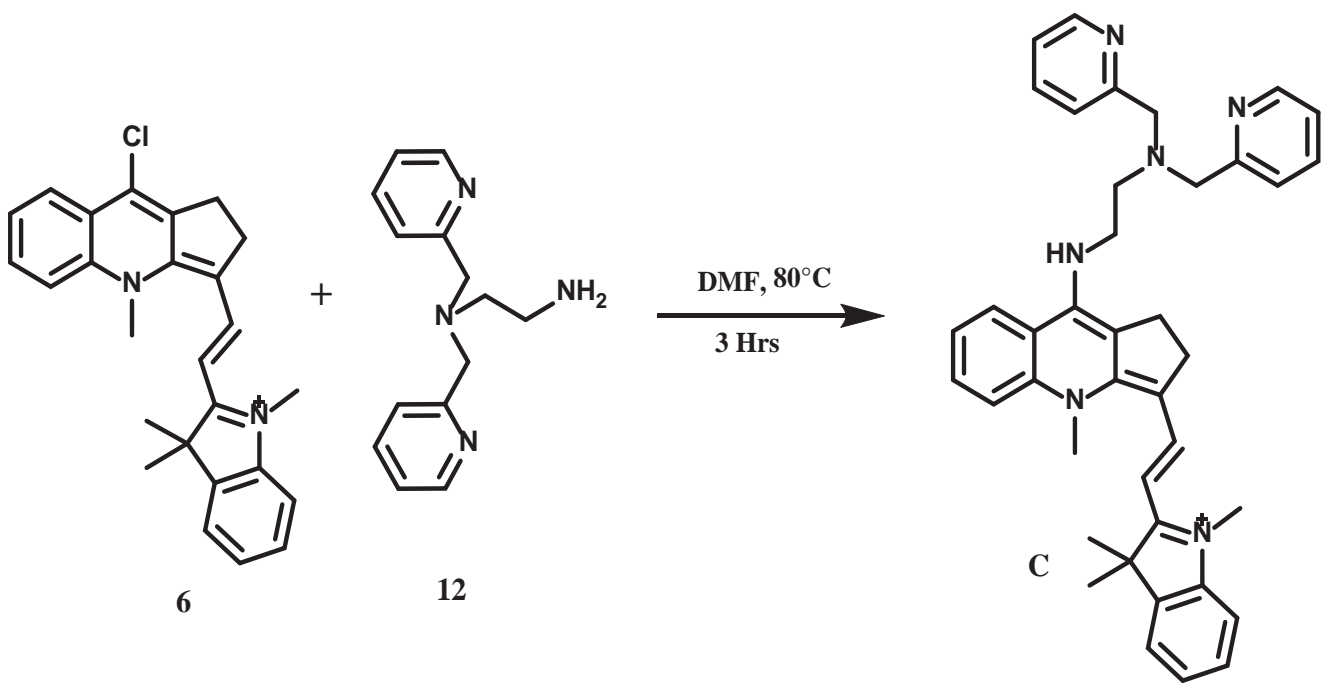

Scheme 4.4. Synthetic route to Compound $\mathrm{C}$

Synthesis of compound C: Compound $\mathbf{6}(0.2 \mathrm{~g}, 0.49 \mathrm{mmol})$ and compound $12(0.47 \mathrm{~g}$, $1.96 \mathrm{mmol}$ ) were dissolved in dry DMF $(40 \mathrm{~mL})$ under nitrogen atmosphere. The reaction mixture was heated to $80^{\circ} \mathrm{C}$ for $3 \mathrm{hrs}$. Distill off the DMF under high vacuum and the crude compound was purified by column chromatography using DCM/EtOH (50/1 to 10/1) to yield a dark blue solid $13(0.09 \mathrm{~g}, 30 \%) .{ }^{1} \mathrm{H}$ NMR $\left(400 \mathrm{MHz}, \mathrm{CDCl}_{3}\right)$ : $\delta$ 8.79-8.77 (d, $J=$ $8.0 \mathrm{~Hz}, 1 \mathrm{H}), 8.64$ (brs, 1H), 8.45-8.44 (d, $J=4.0 \mathrm{~Hz}, 2 \mathrm{H}), 7.95-7.85$ (m, 2H), 7.59-7.48 (m, 4H), 7.41-7.39 (d, $J=8.0 \mathrm{~Hz}, 2 \mathrm{H}), 7.23-7.12(\mathrm{~m}, 4 \mathrm{H}), 6.97-6.93(\mathrm{t}, J=8.0 \mathrm{~Hz}, 1 \mathrm{H})$, 6.77-6.75 (d, $J=8.0 \mathrm{~Hz}, 1 \mathrm{H}), 5.35-5.32(\mathrm{~d}, J=12.0 \mathrm{~Hz}, 1 \mathrm{H}), 4.26(\mathrm{~s}, 3 \mathrm{H}), 4.03(\mathrm{~s}, 6 \mathrm{H})$, 3.39-3.31 (m, 2H), 3.27 (s, 3H), 3.08-3.03 (m, 2H), 2.96-2.90 (m, 2H), $1.61(\mathrm{~s}, 6 \mathrm{H}) .{ }^{13} \mathrm{C}$ NMR (100 MHz, $\left.\mathrm{CDCl}_{3}\right): \delta 164.65,158.71,158.40,150.14,149.30,144.21,141.03$, 139.26, 136.97, 133.54, 133.14, 128.31, 125.82, 125.02, 123.66, 122.62, 122.05, 121.84, $118.90,118.51,116.77,107.63,94.65,59.73,51.96,46.85,42.92,41.51,30.06,29.11$, 28.63, 28.18. HRMS (ESI) calcd for $\mathrm{C}_{40} \mathrm{H}_{43} \mathrm{~N}_{6}[\mathrm{M}]^{+}, 607.8090$; found, 607.3566. 


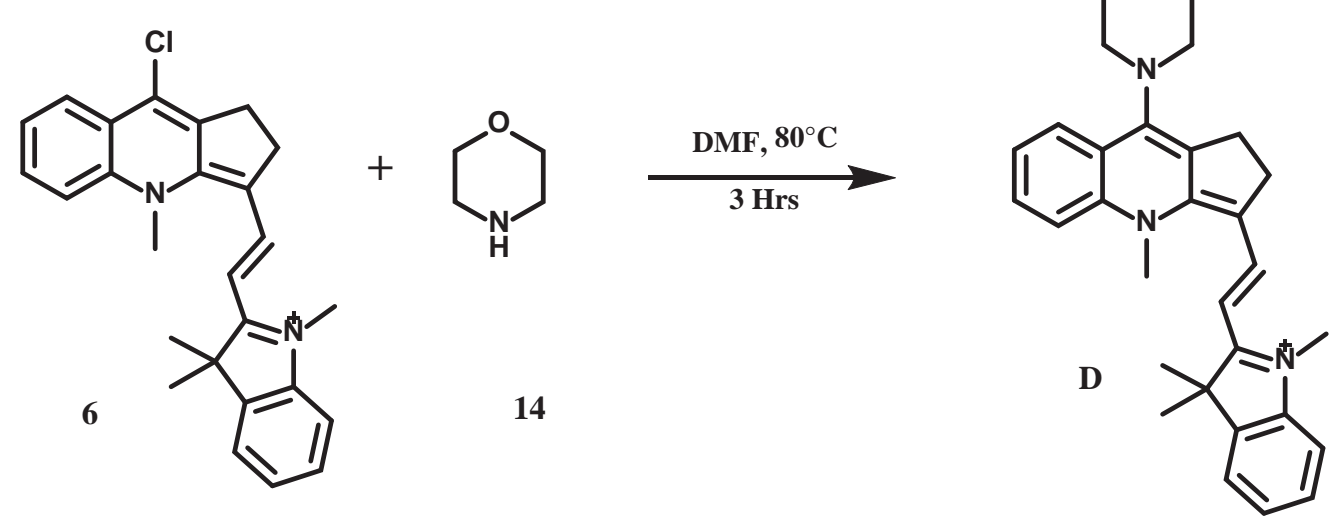

Scheme 4.5. Synthetic route to Compound D

Synthesis of compound D : Compound $\mathbf{6}(0.2 \mathrm{~g}, 0.49 \mathrm{mmol})$ and compound $14(0.39 \mathrm{~g}$, $1.96 \mathrm{mmol})$ were dissolved in dry DMF $(40 \mathrm{~mL})$ under nitrogen atmosphere. The reaction mixture was heated to $80^{\circ} \mathrm{C}$ for $3 \mathrm{hrs}$. Distill off the DMF under high vacuum and the crude compound was purified by column chromatography using DCM/EtOH (50/1 to 10/1) to yield a dark blue solid $15(0.07 \mathrm{~g}, 35 \%) .{ }^{1} \mathrm{H}$ NMR (400 MHz, $\left.\mathrm{CDCl}_{3}\right)$ : $\delta$ 8.04-8.02 (d, $J=$ $8.0 \mathrm{~Hz}, 1 \mathrm{H}), 7.92-7.90(\mathrm{~d}, J=8.0 \mathrm{~Hz}, 1 \mathrm{H}), 7.81-7.72(\mathrm{~m}, 2 \mathrm{H}), 7.52-7.48(\mathrm{t}, J=8.0 \mathrm{~Hz}$, $1 \mathrm{H}), 7.25-7.21(\mathrm{~m}, 2 \mathrm{H}), 7.02-6.99(\mathrm{t}, J=8.0 \mathrm{~Hz}, 1 \mathrm{H}), 6.87-6.85(\mathrm{~d}, J=8.0 \mathrm{~Hz}, 1 \mathrm{H}), 5.51-$ $5.48(\mathrm{~d}, J=12.0 \mathrm{~Hz}, 1 \mathrm{H}), 4.38(\mathrm{~s}, 3 \mathrm{H}), 3.93-3.86(\mathrm{~m}, 4 \mathrm{H}), 3.53-3.47(\mathrm{~m}, 4 \mathrm{H}), 3.37-3.31$ (m, 5H), 3.09-3.04 (m, 2H), 1.63 (s, 6H). ${ }^{13} \mathrm{C}$ NMR (100 MHz, $\left.\mathrm{CDCl}_{3}\right): \delta$ 167.97, 159.72, $153.11,143.65,141.89,139.79,138.20,132.85,130.49,128.48,126.43,125.70,123.27$, $123.17,123.05,122.17,119.02,108.65,96.44,67.55,52.01,47.68,42.97,30.70,29.13$, 28.69, 28.09. HRMS (FAB) calcd for $\mathrm{C}_{30} \mathrm{H}_{34} \mathrm{~N}_{3} \mathrm{O}[\mathrm{M}]^{+}, 452.2700$; found, 452.3000 .

\subsection{Results and conclusion}

Absorption and emission spectra for compounds A, B, C, and D were taken in ethanol. Change in the absorption spectra towards the shorter wavelengths and large strokes shift in compounds $\mathrm{B}, \mathrm{C}$, and $\mathrm{D}$ compared to the compound $\mathrm{A}$ is due to intra molecular charge transfer (ICT) caused by bridge head nitrogen. Molar absorption coefficient and quantum yields for the all the four compounds are calculated in ethanol. 


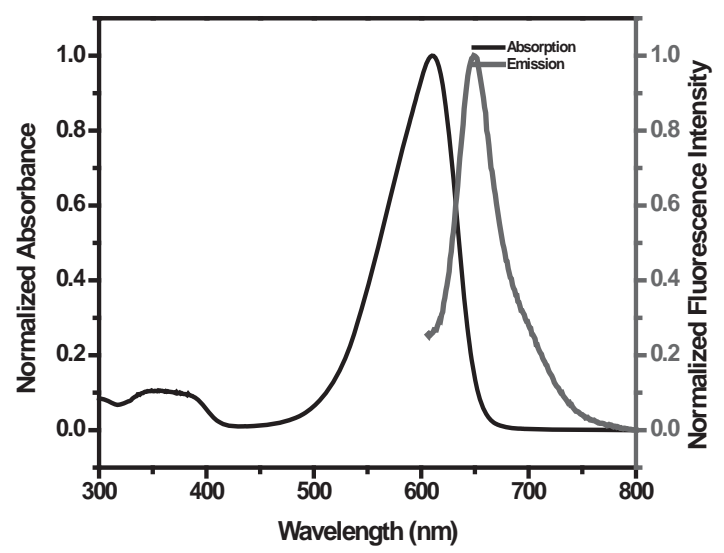

Figure 4.1. Absorption and emission spectra of compound A in ethanol

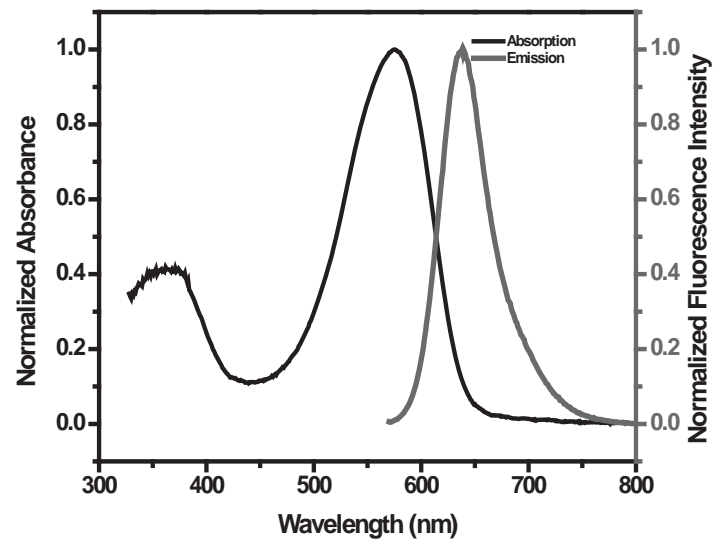

Figure 4.2. Absorption and emission spectra of compound B in ethanol 


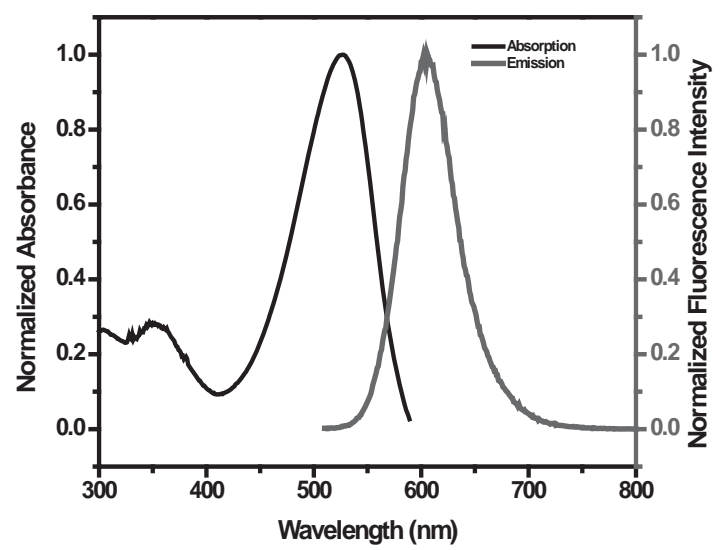

Figure 4.3. Absorption and emission spectra of compound $\mathrm{C}$ in ethanol

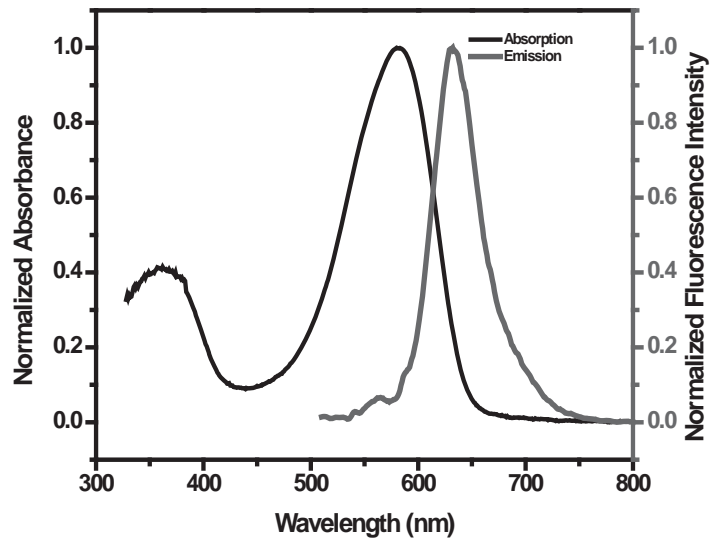

Figure 4.4. Absorption and emission spectra of compound D in ethanol 
Table 4.1. Molar absorption coefficient and quantum yields of compounds A, B, C, and D

\begin{tabular}{|c|c|c|}
\hline Compound & $\begin{array}{c}\text { Molar absorption } \\
\text { coefficient } \mathrm{M}^{-1} \cdot \mathrm{cm}^{-1}\end{array}$ & Quantum yield \\
\hline A & $1.66 \times 10^{4}$ & $22.3 \%$ \\
\hline B & $8.45 \times 10^{3}$ & $8.1 \%$ \\
\hline C & $5.68 \times 10^{4}$ & $0.1 \%$ \\
\hline D & $4.85 \times 10^{3}$ & $5.6 \%$ \\
\hline
\end{tabular}

In conclusion a new hybrid fluorescent dye was prepared and its bridge head chlorine reactivity was tested with primary and secondary amines. This reactivity facilitate the use of this hybrid dye for fluorescent probe design using different bimolecular receptors. 


\section{Chapter 5 : Glycosylated aniline polymer sensor: Amine to imine conversion on protein-carbohydrate binding ${ }^{4}$}

Zhe Wang ${ }^{\text {a }}$, Chunyan Sun ${ }^{\text {a,b }}$, Giri Vegesna ${ }^{c}$, Haiying Liu ${ }^{c}$, Yang Liu ${ }^{\text {d }}$, Jinghong Li ${ }^{d}$, Xiangqun Zeng a,n

a Department of Chemistry, Oakland University, Rochester, MI 48309, United States

b Department of Food Quality and Safety, Jilin University, Changchun 130062, PR China

c Department of Chemistry, Michigan Technological University, Houghton, MI 49931, United States

d Department of Chemistry, Tsinghua University, Beijing 100084, PR China

${ }^{4}$ The material included in this chapter was previously published in Biosensors and Bioelectronics, volume 46, 15 August 2013, pages 183-189 


\subsection{Abstract}

In this report, functionalized mannosylated aniline polymer (manno-PANI) was investigated as an electrochemical platform to study carbohydrate-protein interactions by exploiting the conductivity change of manno-PANI when the specific lectin binding occurs. A systematic study was performed to characterize the interconversion of polyaniline content (from amine to imine) in manno-PANI by UV-vis spectroscopy during its binding with concanavalin A (Con A). Both X-ray photoelectron spectrometry (XPS) and UV-vis results suggest that Con A binding with the manno-PANI film triggers the switching of amine functionalities in the polyaniline backbone, converting them to imine forms. Electrochemical impedance spectroscopy (EIS) was used to quantify the specific interactions between Con A and mannose by measuring the impedance change of mannoPANI film for the detection of Con A. A linear relationship between the impedance and Con A concentration was obtained, and the detection limit reaches to $0.12 \mathrm{nM}$ Con $\mathrm{A}$ in a buffer solution ( $\mathrm{pH}=7.4)$, whereas the addition of nonspecific control lectins to the same manno-PANI film gave very little impedance variations. Stability characterization of the manno-PANI film over 20 weeks shows a maximum drift of only $3 \%$ from the original signal. Thus, the uniquely constructed carbohydrate-PANI hybrid is a promising new carbohydrate recognition moiety for studying carbohydrate-protein interactions, presumably leading to a new electrochemical method for characterization of carbohydrateprotein interactions and carbohydrate-mediated intercellular recognitions.

\subsection{Introduction}

Carbohydrate-protein interactions are abundant in many biological processes including cell-cell communication, signal transduction, host-pathogen recognition, inflammation and tumor cell metastasis ${ }^{1-6}$. When compared to DNA-protein and protein-protein interactions, the carbohydrate- protein interactions are harder to measure due to the lack of chromophores in carbohydrate structures and the presence of multitudes of different carbohydrate monomers, stereoisomers and their combination possibilities. In recent years, advances in the fields of carbohydrate biointerface developments have made 
available a great number of innovative carbohydrate biosensors for characterizing carbohydrate-protein interactions. The most promising approach is to create sensory materials not only containing the carbohydrate receptors for efficient binding with target proteins but also bearing perturbable functionalities for sensitive transduction of recognition events ${ }^{7-9}$. Numerous carbohydrate biosensors were reported by incorporating molecular labels such as fluorophores or biotin either into carbohydrate moiety or protein moiety for detection ${ }^{10-12}$. Fluorescence or biotin labeling requires additional steps. Labeling a biomolecule can drastically change its binding properties and label can also interfere with the true binding process ${ }^{13}$. label-free carbohydrate biosensors in which the binding of carbohydrate with the protein is transduced to a signal that can be measured directly have been demonstrated including dual polarization interferometry ${ }^{14}$, quartz crystal microbalance ${ }^{15-16}$, surface plasmon resonance ${ }^{17-19}$, electrochemical voltammetry ${ }^{20-21}$, $\mathrm{NMR}^{22}$ and isothermal titration calorimetry ${ }^{23}$. However, non-specific interactions such as physical adsorption are serious problems for the label-free carbohydrate biosensors since carbohydrate-protein interactions are typically weaker than protein-protein interactions which often leads to small signal to noise ratio and low sensitivity of the measurements.

Electrochemical methods such as cyclic voltammetry (CV) and electrochemical impedance spectroscopy (EIS) have the capability of measure the weak carbohydrate-protein interactions ${ }^{24-26}$. By using electrochemical probes either conjugated to the carbohydrate moiety or added into the testing solution, they provide a unique electrochemical platform for quantifying carbohydrate-protein interactions ${ }^{27}$. For example, Hu et al. reported an electrochemical biosensor using polyaniline and sugar composites as recognition elements $^{28}$. In their method, aniline was chemically polymerized on the surfaces of carbon nanotubes and D-glucose was modified on the carbon nanotube-polyaniline nanocomposites via Schiff-base reaction subsequently. The use of polyaniline as an electrochemical reporter moiety is elegant, however the conjugation of glucose to polyaniline using Schiff-base reaction could lead to an open ring reaction and the product of Schiff-base reaction is very sensitive to $\mathrm{pH}$ (e.g. lower $\mathrm{pH}$ can result in decomposition of the product). The lack of control of polyaniline and sugar conjugation via Schiff- base reaction and the use of $\mathrm{K}_{3} \mathrm{Fe}(\mathrm{CN})_{6} / \mathrm{K}_{4} \mathrm{Fe}(\mathrm{CN})_{6}$ redox probe in EIS sensing made this 105 
method less practical. Szunerits et al. (2010) characterized the carbohydrate and its complementary lectin binding using EIS by directly coupled carbohydrate to the borondoped diamond electrode terminated with alkynyl func- tional group via copper (I) catalyzed azide alkyne cycloaddition click reaction. This carbohydrate biointerface fabrication still requires several steps and the amount of carbohydrate receptors incorporated is monolayer which may limits its sensitivity and dynamic range.

Here we report a new and better electrochemical based label free carbohydrate biosensor in which a well-defined aniline monomer bearing a-mannoside residue at ortho-position of the aniline ring enables for one-step electrochemical polymerization of the glycosylated aniline polymer (manno-PANI) on the electrode substrate with controlled thickness and porosity (Scheme 5.1 and Scheme D.1). The glycosylated aniline polymer was shown to maintain the bioactivity of the natural sugar and can directly transduce the carbohydrateprotein binding to a change of the electrical signal (impedance by EIS or current by $\mathrm{CV}$ ) due to the extremely sensitivity of the polyaniline to its protonation/deprotonation states (Scheme 5.1). Multiple techniques including X-ray photoelectron spectroscopy (XPS), scanning electron microscopy (SEM), UV-vis spectroscopy, CV have been used to characterize the electrochemical polymerization of the glycosylated aniline and its electrochemical properties. Quantitative characterization of analytical parameters of the manno-PANI as a biosensor for detection of Con A by EIS shows high sensitivity, selectivity and stability validating its use as a new carbohydrate recognition moiety for studying carbohydrate-protein interaction and for label free carbohydrate based biosensor development. (Insert Scheme 5.1)

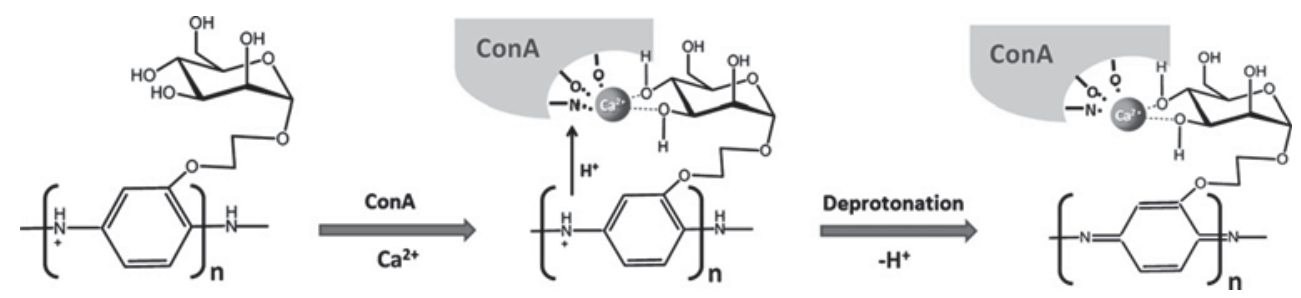

Scheme 5.1. Deprotonation of mannosylated polyaniline (PANI) during the Con A binding. 


\subsection{Materials and methods}

\subsubsection{Materials}

Indium tin oxide (ITO) glass slides (CD-501N-CUV) were purchased from Delta Technologies Co. Ltd. Concanavalin A (Con A), peanut agglutinin (PNA), Elderberry lectin (SNA), Ulex euro- paeus agglutinin (UEA), Wheat germ agglutinin (WGA), Pisum sativum agglutinin (PSA) were purchase from Sigma-Aldrich or FluoProbess/Interchim Co. Ltd. All reagents and material were analytical grade, and solvents were purified by standard procedures. (4-(2-hydroxyethyl)-1piperazineethanesulfonic acid) (HEPES) was purchased from Sigma-Aldrich.

\subsubsection{Synthesis and characterization of glycosylated aniline polymer film}
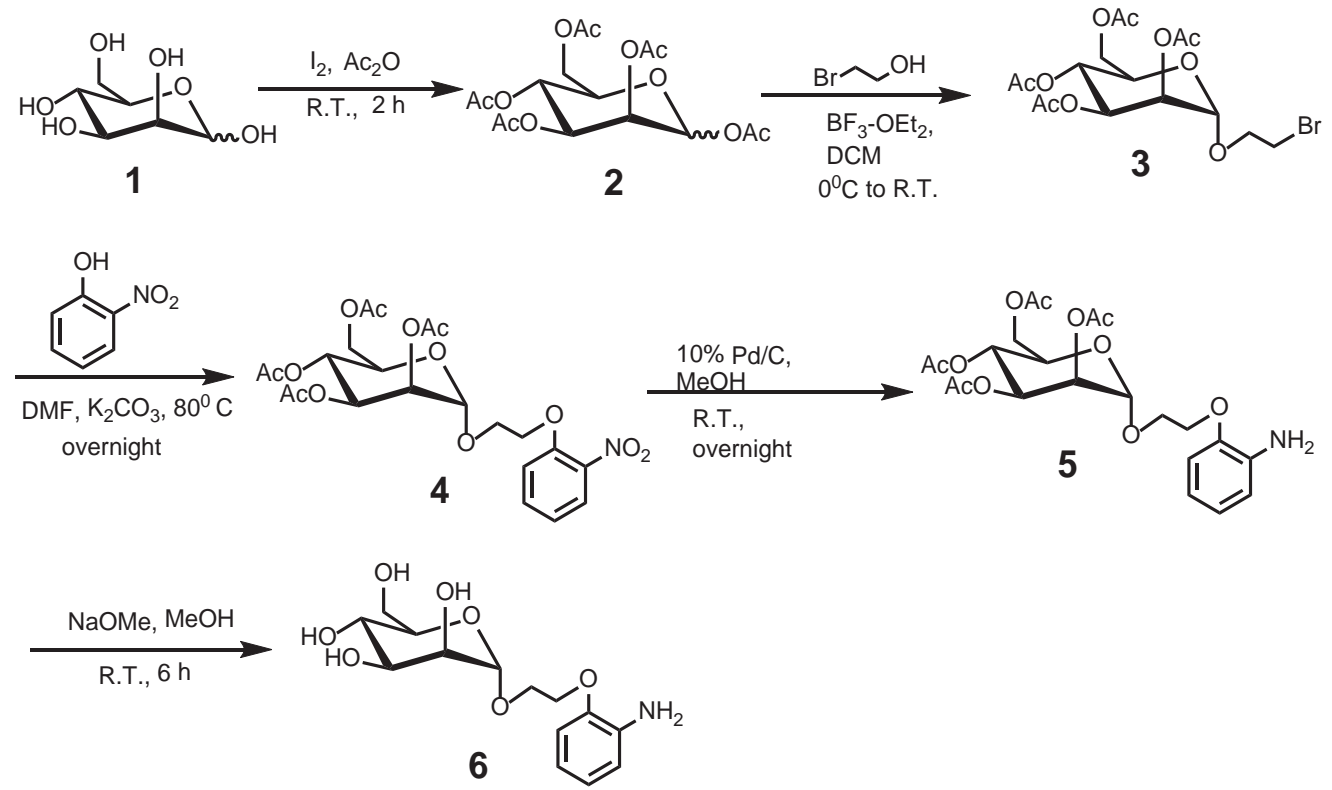

Scheme 5.2. Synthetic route to a glycosylated aniline monomer.

1,2,3,4,6-Penta-O-acetylmannose 2: Compound 2 was prepared according to the following procedure. Iodine $(250 \mathrm{mg})$ was added to the suspension of D-mannose (1) (5 g, $0.027 \mathrm{~mol})$ 
in acetic anhydride $(50 \mathrm{~mL})$. Reaction mixture began to warm which is a sign for the initiation of acetylation and sugar started dissolving in the solution. After the reaction was conducted at room temperature for 30 minutes, iodine was quenched by aqueous sodium thiosulphate solution, and the crude product was extracted into dichloromethane $(2 \times 60 \mathrm{ml})$ and washed with aqueous sodium carbonate solution, and brine solution. The organic layer was collected, dried with sodium sulphate and concentrated under reduced pressure to get the crude product (10 g, 92\%) for the next step without further purification.

1-O-bromoethyl-2,3,4,6-tetra-O-acetyl- $\alpha$-D-mannose 3: compound 2 (5 g, $0.012 \mathrm{~mol})$ and bromoethanol $(1.09 \mathrm{~mL}, 0.015 \mathrm{~mol})$ were dissolved in dry dichloromethane $(40 \mathrm{~mL})$ in three-necked 150-mL flask under nitrogen atmosphere. Then $\mathrm{BF}_{3}-\mathrm{OEt}_{2}(8.4 \mathrm{~mL}, 0.066$ mol) was added drop wise to the flask by using addition funnel at $0{ }^{\circ} \mathrm{C}$. The resulting mixture was allowed to come to room temperature and stirred for 12 hours. After the mixture was cooled down to $0{ }^{\circ} \mathrm{C}$ and slowly quenched by cold aqueous $\mathrm{NaHCO}_{3}$ solution, it was poured into dichloromethane $(30 \mathrm{~mL})$ and washed with aqueous $\mathrm{NaHCO}_{3}$ solution, and brine, respectively. The organic layer was dried over anhydrous $\mathrm{Na}_{2} \mathrm{SO}_{4}$ and concentrated under a reduced pressure to yield a light brown oily compound. Recrystallization of the crude product in $\mathrm{Et}_{2} \mathrm{O}: \operatorname{Hexane}(1: 5, \mathrm{v} / \mathrm{v})$ gave a white crystal compound (3.5 g, 60\%). ${ }^{1} \mathrm{H}$ NMR (400 MHz, $\mathrm{CDCl}_{3}$ ): $\delta 5.34-5.23$ (m, 3H), $4.85(\mathrm{~s}, 1 \mathrm{H})$, 4.26-4.22 (m, 1H), 4.12-4.09 (m, 2H), 3.98-3.83 (m, 2H), 3.49 (t, j=5.6 Hz, 2H), 2.13 (s, $3 \mathrm{H}) 2.08(\mathrm{~s}, 3 \mathrm{H}), 2.02(\mathrm{~s}, 3 \mathrm{H}), 1.98(\mathrm{~s}, 3 \mathrm{H}) .13 \mathrm{C} \mathrm{NMR}\left(100 \mathrm{MHz}, \mathrm{CDCl}_{3}\right): \delta$ 170.8, 170.2, $170.0,169.9,97.9,69.6,69.2,69.1,68.6,66.2,62.6,29.7,21.0,20.9,20.8$.

(2R,3R,4S,5S,6S)-2-(acetoxymethyl)-6-(2-(2-nitrophenoxy)ethoxy)tetrahydro-2H-pyran3,4,5-triyl triacetate $4: 2$-Nitrophenol $(0.61 \mathrm{~g}, 0.004 \mathrm{~mol})$ and potassium carbonate $(0.72$ g, $0.005 \mathrm{~mol}$ ) was added to dimethylformamide (DMF) $(20 \mathrm{~mL})$ containing the compound $3(2 \mathrm{~g}, 0.004 \mathrm{~mol})$ under a nitrogen atmosphere. The reaction mixture was heated to $80{ }^{\circ} \mathrm{C}$ overnight and the reaction was monitored by TLC. After the complete consumption of starting materials was verified by TLC, DMF in the mixture was removed under the reduced pressure. When the mixture was poured into dichloromethane $(60 \mathrm{ml})$ and washed with water and with brine, respectively, the organic layer was collected, dried over 
anhydrous $\mathrm{Na}_{2} \mathrm{SO}_{4}$, and concentrated under reduced pressure to get a colorless crude product. The crude product was purified by column chromatography using EtOAc: hexane $(1: 1, \mathrm{v} / \mathrm{v})$ to yield a colorless compound $(1.8 \mathrm{~g}, 81 \%) .{ }^{1} \mathrm{H} \mathrm{NMR}\left(400 \mathrm{MHz}, \mathrm{CDCl}_{3}\right): \delta 7.75$ (m, 1H), $7.49(\mathrm{~m}, 1 \mathrm{H}), 7.06(\mathrm{~m}, 1 \mathrm{H}), 6.99(\mathrm{~m}, 1 \mathrm{H}), 5.24-5.20(\mathrm{~m}, 3 \mathrm{H}), 4.90(\mathrm{~s}, 1 \mathrm{H}), 4.30-$ 4.19 (m, 3H), 4.08-4.01 (m, 3H), 3.86-3.81 (m, 1H), 2.09 (s, 3H) $2.03(\mathrm{~s}, 3 \mathrm{H}), 1.98(\mathrm{~s}, 3 \mathrm{H})$, 1.91(s, 3H). ${ }^{13} \mathrm{C}$ NMR (100 MHz, $\left.\mathrm{CDCl}_{3}\right): \delta 170.8,170.2,170.0,169.9,152.0,140.2$, 134.3, 125.7, 121.0, 114.8, 97.8, 97.7, 69.6, 69.4, 69.3, 69.1, 68.8, 68.6, 68.5, 66.3, 66.1, $65.9,65.8,62.6,62.5,21.0,20.9,20.8,20.7$.

(2R,3R,4S,5S,6S)-2-(acetoxymethyl)-6-(2-(2-aminophenoxy)ethoxy)tetrahydro-2Hpyran-3,4,5-triyl triacetate 5: Compound 4 (1.5 g, $0.002 \mathrm{~mol})$ was added to methanol (20 $\mathrm{ml}$ ) containing $10 \% \mathrm{Pd} / \mathrm{C}(0.3 \mathrm{~g})$. Hydrogen gas was applied to the reaction mixture through a balloon and the reaction mixture was stirred overnight. After $\mathrm{Pd} / \mathrm{C}$ was filtered through a short celite pad, methanol was removed from the mixture under a reduced pressure. The crude product was purified through column chromatography using EtOAc: hexane $(7: 3, \mathrm{v} / \mathrm{v})$ to produce a colorless product $(1.2 \mathrm{~g}, 85 \%)$. ${ }^{1} \mathrm{H}$ NMR $\left(400 \mathrm{MHz}, \mathrm{CDCl}_{3}\right)$ : $\delta$ 6.77-6.74 (m, 2H), 6.68-6.61 (m, 2H), 5.30-5.22 (m, 3H), $4.88(\mathrm{~s}, 1 \mathrm{H}), 4.23-4.13(\mathrm{~m}$, 3H), 4.01-3.96 (m, 3H), 3.85-3.80 (m, 3H), 2.10 (s, 3H), 2.02 (s, 3H), 1.98 (s, 3H), 1.94(s, $3 \mathrm{H}) .{ }^{13} \mathrm{C} \mathrm{NMR}\left(100 \mathrm{MHz}, \mathrm{CDCl}_{3}\right): \delta 170.8,170.2,170.0,169.9,149.4,130.3,121.8,116.4$, $109.1,97.9,69.6,69.0,68.9,67.5,66.5,66.3,62.6,21.0,20.9,20.8,20.7$. HRMS (FAB) calcd for $\mathrm{C}_{22} \mathrm{H}_{29} \mathrm{NO}_{11}[\mathrm{M}]^{+}, 483.17$; found, 483.17 .

(2S,3S,4S,5S,6R)-2-(2-(2-aminophenoxy)ethoxy)-6-(hydroxymethyl)tetrahydro-2Hpyran-3,4,5-triol 6: $\mathrm{NaOMe}(0.22 \mathrm{~g}, 0.004 \mathrm{~mol})$ was added to methanol $(15 \mathrm{~mL})$ containing compound $5(1 \mathrm{~g}, 0.002 \mathrm{~mol})$ at room temperature. After the complete consumption of the starting material was verified by TLC, the methanol was removed from the mixture under a reduced pressure. The crude compound was purified through column chromatography using MeOH: dichloromethane (1:5, v/v) to yield the product (0.39 g, 60\%). ${ }^{1} \mathrm{H}$ NMR (400 $\left.\mathrm{MHz}, \mathrm{CD}_{3} \mathrm{OD}\right): \delta$ 6.83-6.81 (m, 1H), 6.76-6.71 (m, 2H), 6.67-6.63 (m, 1H) $4.84(\mathrm{~d}, \mathrm{~J}=1.6$ $\mathrm{Hz}, 1 \mathrm{H}), 4.15-4.13(\mathrm{~m}, 2 \mathrm{H}), 4.06-4.01(\mathrm{~m}, 1 \mathrm{H}), 3.84-3.78(\mathrm{~m}, 3 \mathrm{H}), 3.71-3.66(\mathrm{~m}, 2 \mathrm{H}), 3.61-$ 3.57 (m, 2H). ${ }^{13} \mathrm{C}$ NMR (100 MHz, $\left.\mathrm{CD}_{3} \mathrm{OD}\right): \delta 147.0,136.7,121.4,118.6,115.8,112.4$, 
100.5, 73.5, 71.3, 70.9, 67.9, 67.4, 66.0, 61.7. HRMS (FAB) calcd for $\mathrm{C}_{14} \mathrm{H}_{22} \mathrm{NO}_{7}[\mathrm{M}+\mathrm{H}]^{+}$, 316.13; found, 316.14 .

The manno-PANI was synthesized following a six-step method with details presented in here. Electrochemical experiments were carried out using VersaSTAT MC (Princeton AMETEK, US) instrument. Manno-PANI was made by electrodeposition on the ITO slide as the working electrode (sensing area is $\left.2.8 \mathrm{~cm}^{2}\right)$ in HEPES $(\mathrm{pH}=7.4)$ buffer containing $20 \mathrm{mM}$ monomer of mannose functionalized aniline by cyclic voltammetry (CV). $\mathrm{CV}$ was performed between $0.2 \mathrm{~V}$ and $1.2 \mathrm{~V}$ versus $\mathrm{Ag} / \mathrm{AgCl}$ reference electrode at $50 \mathrm{mV} / \mathrm{s}$. Nitrogen was purged into the system to remove the oxygen in the electrolytes. Pt wire was used as the counter electrode. Before further measurements, the manno-PANI modified electrode was rinsed by HEPES to remove unbound lectins and then dried by high purity nitrogen flow. And then, the electrodes were immersed and incubated in HEPES containing different concenrations of lectins and $2 \mathrm{mM} \mathrm{Ca}^{+2}$ in variable time (from $0 \mathrm{~min}$ to $600 \mathrm{~min}$ ) for further characterizations. Con $\mathrm{A}$ is a homotetramer with each sub-unit (26.5 KDa, 235 amino-acids, heavily glycated). Divalent cations (usually $\mathrm{Mn}^{+2}$ and $\mathrm{Ca}^{+2}$ ) must be present to permit the binding of Con A to carbohydrates because they are necessary in order to get an active Con A conformation. $\mathrm{Ca}^{+2}$ is used in all the lectins and manno-PANI binding experiments ${ }^{29}$.

The surface morphology of modified electrodes was characterized by scanning electron microscopy (SEM) (JSM-6510GS from JEOL), operating with an accelerating voltage of $20 \mathrm{kV}$. XPS spectra were obtained with a PHI 5000 VersaProbe X-ray photoelectron spectrometer. A monochromatic Al-KR X-ray source $(1486.7 \mathrm{eV})$ was used. N $1 \mathrm{~s}$ of XPS core level spectra were acquired with a pass energy of $20 \mathrm{eV}$, a step of $0.1 \mathrm{eV}$, and a dwell time of $200 \mathrm{~ms}$. The takeoff angle between the sample film surface and the photoelectron energy analyzer was 90 degree. The typical operating pressure was around $5 \times 10^{-10}$ Torr in the sample chamber. The Shirley function was used as a background and GaussianLorentzian cross- products were used to fit the individual peaks $\mathrm{s}^{30-31}$. UV-vis spectra of the manno-PANI modified ITO electrodes were recorded at the open circuit potential using a Cary 100 Bio spectrophotometer (Varian). The modified ITO slides were placed in the 
light path so that the transmitted light passing directly through it and all spectra were calibrated by a baseline of neat ITO. EIS experiments were carried out under open circuit potential using VersaSTAT MCinstrument as well. All experiments were performed at room temperature $(298 \mathrm{~K})$.

\subsubsection{Formation and characterization of mannosylated aniline polymer film}

Mannosylated aniline monomer was electrochemically polymerized to form mannoPANI on the ITO electrode in HEPES $(\mathrm{pH}=7.4)$ buffer as reported in neutral aqueous media ${ }^{32-37}$. At $\mathrm{pH}$ (7.4), carbohydrates are uncharged and their bioactivities are maintained. The HEPES buffer contains bulky zwitterionic 4-(2-hydroxyethyl)-1piperazineethanesulfonic acid which is deliberately chosen for several reasons. First, the bulky ion of HEPES will minimize its migration as the counter ion within the aniline polymer film for the charge compensation when mannosylated aniline monomer is electrochemically oxidized to form manno-PANI. This will result in an emeraldine (halfoxidized) manno-PANI formation rather than fully oxidized pernigraniline form. The emeraldine form of manno-PANI is beneficial for impedance-based detection of its binding with Con A for several reasons. First, it contains protonated amine functionality; second, if binding of Con A to manno-PANI can lead to the change of the manno-PANI oxidation states due to deprotonation, the bulky ions of HEPES will not be able to quickly compensate the charge, thus any change of the conductivity of the manno-PANI film can be ascribed to the lectin-carbohydrate binding events. This strategy significantly reduces the noise and increases the signal to noise ratio for sensitive detection of mannose-Con A interactions by EIS readout. Finally, HEPES buffer is better than PBS buffer in lectin binding reaction because PBS buffer can react with trace metal ions such as $\mathrm{Ca}$ (II) to form calcium phosphate precipitates. Similarly to other alkyl substituted aniline polymerization, the steric hindrance of the mannose substituent in aniline monomer can induce torsion angle between phenyl rings in the polymer backbone during electrochemical polymerization process. Subsequently, it reduces the p-conjugation in the polyaniline chain and destabilizes the polysemiquinone radical cation form ${ }^{38}$. The stability of the polysemiquinone radical cation is decreased in the substituted derivatives to the extent that 
the reduced amine form of the polymer is directly oxidized to the imine form, and that the semiquinone form has no detectable existence under the conditions employed in the CV studies. As shown in (Fig. D.1), only one pair of redox peaks located at $0.38 \mathrm{~V}$ and $0.25 \mathrm{~V}$ was observed in the CV. This result is consistent with the characteristics of substituted aniline that is partially oxidized to form the emeraldine $\mathrm{PANI}^{39-40}$. In neutral media, PANI could grow with the same efficiency as in acidic media ${ }^{33}$, although there was only one pair of redox peaks in $\mathrm{CV}^{41}$. The surface coverage of the manno-PANI is $1.71 \quad 107 \mathrm{M} \mathrm{cm}$ 2 calculated based on the charge from the CV curves ${ }^{42-43}$. Since the aniline was covalently bonding with mannose substituent in a ratio of $1: 1$, the total amount of sugar is about $479 \mathrm{nM}$ on the ITO electrode.

\subsection{Results and discussion}

\subsubsection{Characterization of mannosylated polyaniline film}

The manno-PANI modified ITO electrodes were characterized by $\mathrm{CV}$ in the absence and the presence of Con A at various concentrations (Fig. 5.1). In the presence of Con A, both anodic and cathodic peak currents decreased. The decreased current could be ascribed to the binding of Con A to the manno-PANI. In comparison to the mannose complementary lectin Con A, the other control lectins (Fig. D.2) gave much less current decrease due to non-specific interactions. The binding of Con A to the manno-PANI could increase the interchain distance of the polymer chains and partially block the electron-transfer process among the manno-PANI ${ }^{2,44-46}$ from half oxidized emeraldine state to the fully oxidized pernigraniline state. Since the counterion is very big, we can neglect the counterion transfer to the manno-PANI and attribute the changes of the conductivity of manno-PANI to Con A binding only. Interestingly, a positive shift of anodic peak potential is observed with the increase of Con A concentrations but no potential shift for cathodic peak is observed. We rationalize that the initiate state of polyaniline is emeraldine form, and parts of emeraldine were oxidized after its binding to Con A. This rationalization was further supported by in-situ UV-vis absorption spectra and XPS results below. (Insert Fig. 5.1) 
Surface morphology of manno-PANI was investigated by SEM. Fig. 5.2 (a) shows the SEM image of manno-PANI on the ITO electrode. The electrochemically synthesized manno-PANI shows a textile structure which is different from the flower-like morphology observed in unsubstituted polyaniline films ${ }^{47-48}$. This is consistent with the $\mathrm{CV}$ results in Fig. D.1 that the steric hindrance of glycosylated aniline monomer affects polymer- ization of aniline. As shown in Fig. 5.2(a), the white spots on the surface are likely due to the electron aggregation at mannose functional groups. Fig. 5.2(b) shows that after the manno-PANI film was treated with Con A solution, the manno-PANI film morphology changed to a sponge-like architecture, suggesting a complete binding of manno-PANI to Con A. We verified the presence of Con A by further adding Escherichia coli W1485 (Fig. 5.2c) since the lipopoly- saccharides (LPS) of the O-antigen of E. coli W1485 have glucose subunit which can bind selectively with Con A. ${ }^{16,49-50}$ As shown in Fig. 5.2(c) and 5.2d), E. coli bacteria were observed as uniformly distributed on the manno-PANI-Con A film, further proving the specificity of binding between manno-PANI and Con A. (Insert Fig. 5.2)

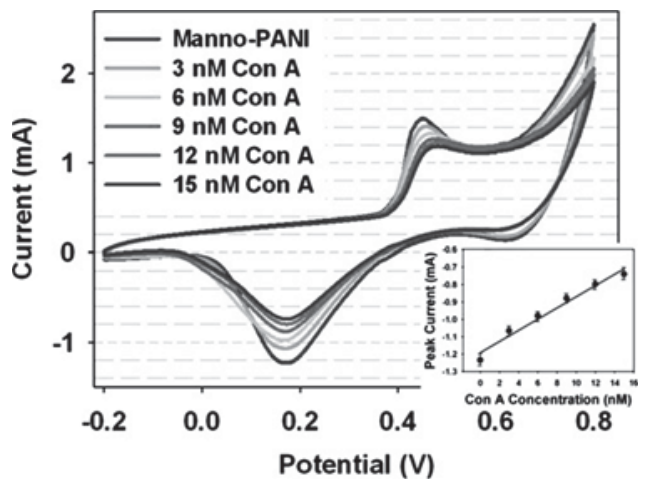

Figure 5.1. Cyclic voltammograms of manno-PANI film in the absence and presence of Con A at different concentrations in HEPES buffer with $2 \mathrm{mM} \mathrm{Ca}^{+2}$. Scan rate was 50 $\mathrm{mV} / \mathrm{s}, \mathrm{Ag} / \mathrm{AgCl}$ was used as a reference electrode, and Pt wire was used as a counter electrode. 

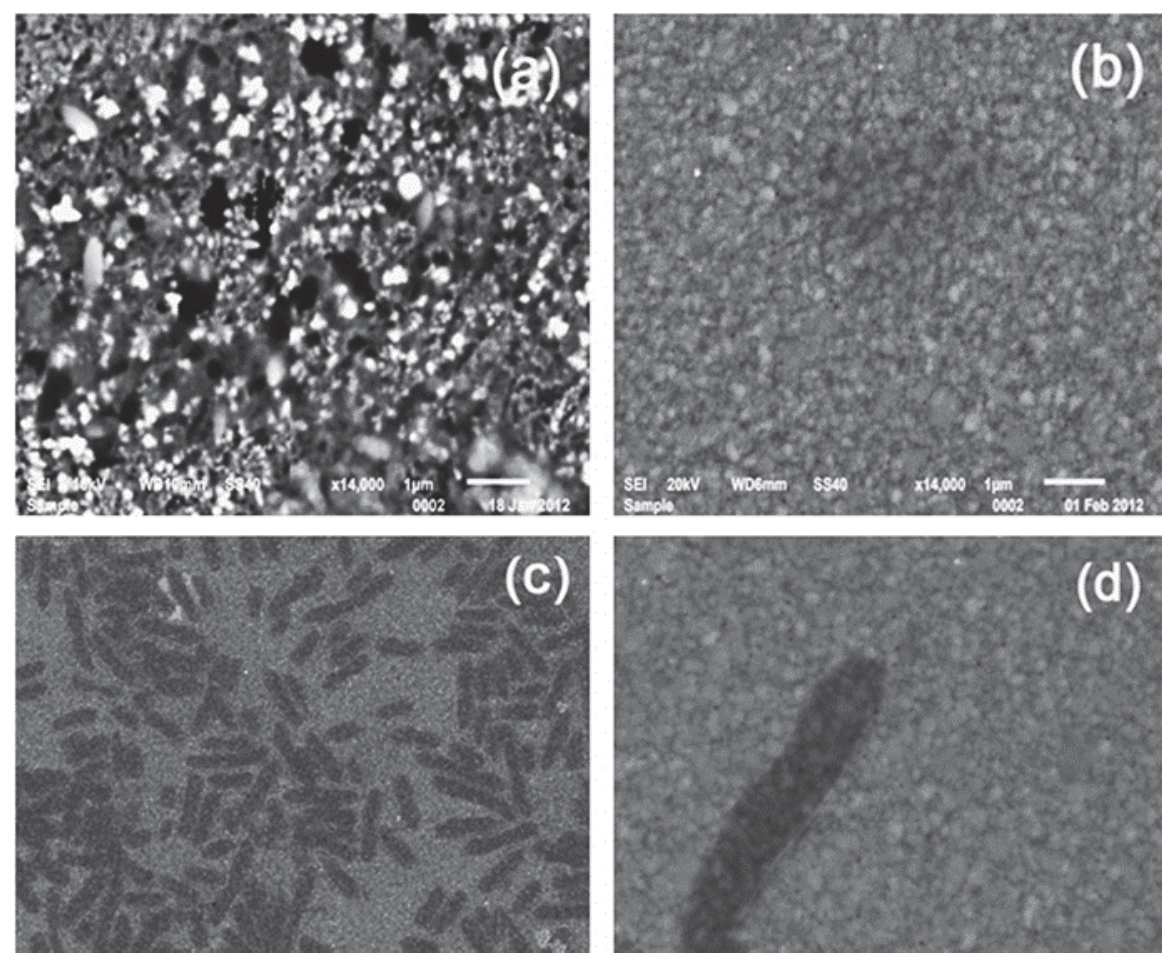

Figure 5.2. The SEM images of mannosylated polyaniline film on the ITO electrode in the absence (a) and presence (b) of $15 \mathrm{nM} \mathrm{Con} \mathrm{A,} \mathrm{(c)} \mathrm{and} \mathrm{(d)} \mathrm{in} \mathrm{the} \mathrm{presence} \mathrm{of} \mathrm{Escherichia}$ coli after the experiment in (b).
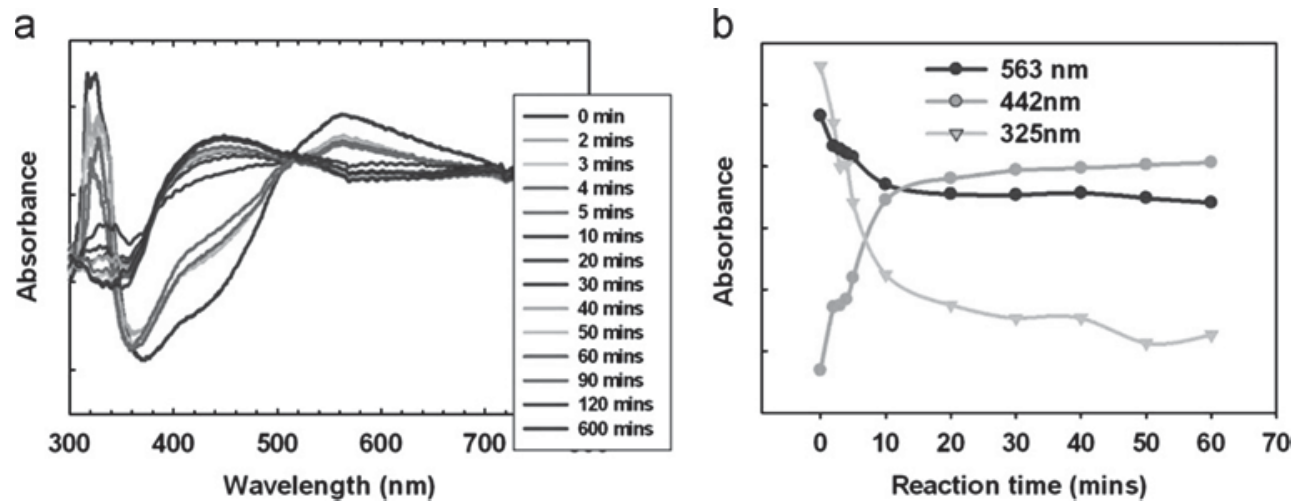

Figure 5.3. (a) UV-visible absorption spectra of manno-PANI on the ITO electrodes obtained for a period of time when $15 \mathrm{nM}$ Con A was added. The ITO electrodes were washed with HEPES buffer each time before the measurement. (b) Correlation of 
absorbance vs. binding time of Con A to manno-PANI at $325 \mathrm{~nm}, 442 \mathrm{~nm}$ and $563 \mathrm{~nm}$ wavelength.

\subsubsection{Analysis of Con A binding process by UV-vis absorption spectroscopy}

The manno-PANI and its interaction with Con A were characterized by the UV-vis absorption spectroscopy. Consistent with the interpretation of electrochemical CV study in Figs. 5.2 and 5.3, the manno-PANI in the absence of Con A shows two absorbance maxima at $325 \mathrm{~nm}$ and $563 \mathrm{~nm}$ respectively. These are the characteristics of emeraldine form of PANI. The binding of Con A to the manno-PANI causes an increase of absorbance band at $442 \mathrm{~nm}$ (polaron) and the decreases of the absorbance bands at 325 $\mathrm{nm}\left(\mathrm{p}-\mathrm{pn}\right.$ transition) and $563 \mathrm{~nm}$ (bipolaron (doped)). The isosbestic point ${ }^{51}$ at $510 \mathrm{~nm}$ confirms that theemeraldine form of manno-PANI changes to the pernigraniline form of PANI when sufficient Con A has been added ${ }^{51-52}$. This phenomenon also was shown in the absorption spectra of polyaniline and substituted polyaniline when the $\mathrm{pH}$ was changed from low (two) to high (nine) $)^{53-54}$. The possi- bility of degradation of mannoPANI is unlikely since there is no new absorption band emerging ${ }^{55}$. The UV-vis absorption spectra of manno-PANI binding to Con A shows the same trends as substituted PANI when the $\mathrm{pH}$ is increased from 2 to 9 , suggesting that binding of Con A to manno-PANI leads to deprotonation of manno-PANI from emeraldine state to perni- graniline state. Carbohydrates are weak acids with pKa values ranging from 12 to 14 (e.g. galactose 12.39, glucose 12.28, xylose 12.15, mannose 12.08 and fructose 12.03). Thus, carbohydrate- protein interaction would be sensitive to local $\mathrm{pH}$ change. The binding of a carbohydrate to a protein often involves a subtle conformational change of protein that can result in the protona- tion/deprotonation of the protein ${ }^{56}$. In the presence of metal ion $\left(\mathrm{Mn}^{+2}, \mathrm{Ca}^{+2}\right)$, ion and proton exchange could affect the binding of carbohydrate to the protein ${ }^{57-58}$. As shown in Scheme 5.1, if the proton switching occurs during the binding of carbohydrate to protein, it will lead to changes of local charge distribution of the

polyaniline ${ }^{10,59}$. We further characterized the oxidation state of manno- PANI with and without Con A binding by XPS study below (Fig. 5.4). (Insert Fig. 5.3) 

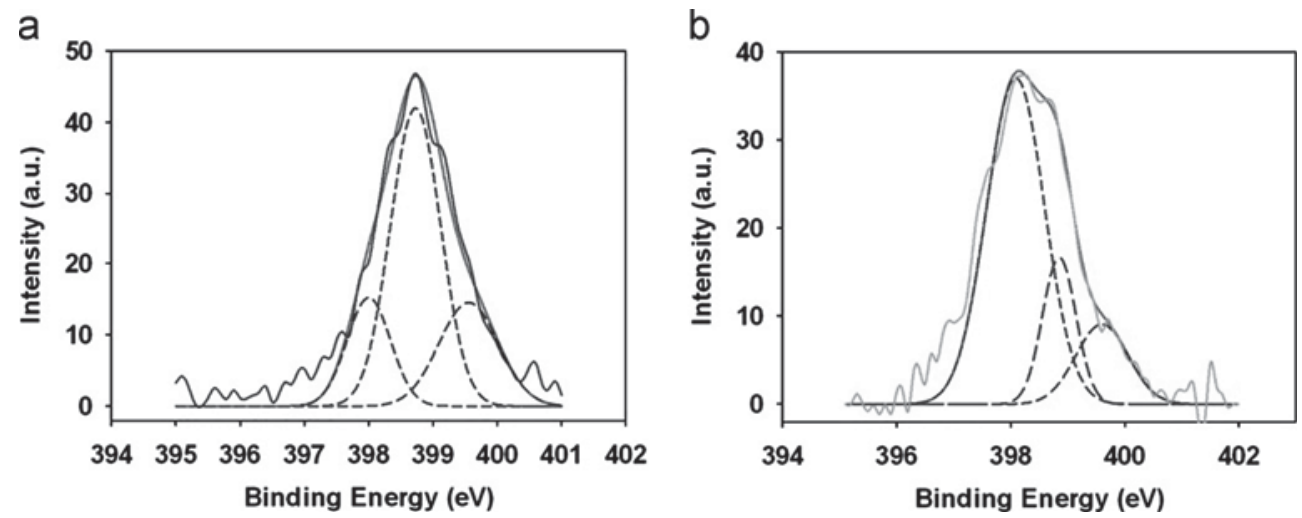

Figure 5.4. High-resolution N1s XPS core-level spectra of (a) manno-PANI and (b) after manno-PANI interaction with $15 \mathrm{nM}$ Con A in HEPES. The brown solid lines are the XPS spectra and the red solid lines are for the total fits, blue dash lines are for the component fitted peaks for imine, amine and the radical cationic nitrogen atom. (For interpretation of the references to color in this figure legend, the reader is referred to the web version of this article.)

\subsubsection{Characterization of the energy level of the nitrogen elements in polyaniline by XPS}

XPS was used to characterize the change of chemical composition of manno-PANI upon its binding to Con A. The XPS was used to analyze quantitatively the nitrogen oxidation states to understand the chemical microenvironment change of manno-PANI when it binds to Con A. Typically the protonation occurs preferentially at the imine sites of PANIchain ${ }^{60}$. Fig. 5.4 shows the N1s XPS spectra of manno-PANI before and after interactions with Con A. It is worth noting that the signal consists of three compo- nents corresponding to three different nitrogen species, i.e imine, amine and radical cationic nitrogen respectively after deconvolution of the XPS spectra. The one centered at $398.8 \mathrm{eV}$ is attributed to the protonated nitrogen atom $(-\mathrm{NH}-)$ coming from the amine group in the emeraldine form ${ }^{35}$, 61-62 typically the binding energy of amine nitrogen (-NH-) in polyaniline is higher than $399.0 \mathrm{eV}$. The reduced binding energy of nitrogen in -NH- of manno-PANI is likely due to the mannosesubstituents ${ }^{63}$. The binding energy of imine nitrogen $(-\mathrm{N}=)$ as a result of 
amine deprotonation in the quinone structure of manno-PANI is at $398.0 \mathrm{eV}$. There is also some signals above $399.5 \mathrm{eV}$ expected from the higher oxidization state of nitrogen element, (the radical cationic nitrogen $-\mathrm{N} \bullet{ }^{+}-$) in the $f\left(\mathrm{~m}^{16,35,62}\right.$. It is $-\mathrm{N} \bullet{ }^{+}$- which results from the protonation of the quinonoid imine in the ionic environment ${ }^{30-31,35}$. As shown in Fig. 5.4b, after interactions with Con A, the nitrogen binding energy peaks of manno-PANI shift to lower binding energy states. In manno-PANI, the atomic concentrations of amine (-NH-) account for about 58.6\% and imine accounts of $18.3 \%$. After interactions with Con $\mathrm{A}$, content of proto- nated nitrogen (-NH-) decreased to $17.6 \%$ and the imine nitrogen $(-\mathrm{N}=)$ increases to $67.5 \%$. The content of the radical cationic nitrogen also decreased from $23.1 \%$ to $14.9 \%$. It confirms that most of emeraldine has been converted to pernigraniline on PANI skeleton. In the protonated form of mannoPANI, emeraldine form of polyaniline has highest conductivity among various oxidation states of $\mathrm{PANI}^{64}$, since the free doublet electrons on the nitrogen atoms participate in the formation of orbitals delocalized along the polymer chain. However quinoid form is unstable in non-ionized state. Pernigraniline shows very low conductivity due to the lack of disorder in PANI. A marginal degree of protonation is sufficient to induce the conductorinsulator transition upon ConA binding. (Insert Fig.5.4)

In summary, the significant morphological and spectral change of manno-PANI observed by SEM images and UV-vis absorption spectra, respectively and the changes of nitrogen binding energy upon addition of Con A support that trace amount of Con A binding to mannose residues in the manno-PANI can significantly trigger polyaniline electronic microenvironment changes which can be used for the sensitive detection of specific carbohydrate- protein interaction at very low protein concentration.

\subsubsection{Quantification of mannosylated polyaniline-protein interactions by electrochemical techniques for sensor application}

In EIS, the electrode-solution interface is perturbed with an alternating signal of very small magnitude, thus, EIS can be considered non-invasive for measurements of the conductivity change. Fig. 5.5a shows the Bode plot of manno-PANI at various Con A 
concentrations. The electrochemical impedance-frequency plot (Bode plot) is more practical to apply in sensor system.
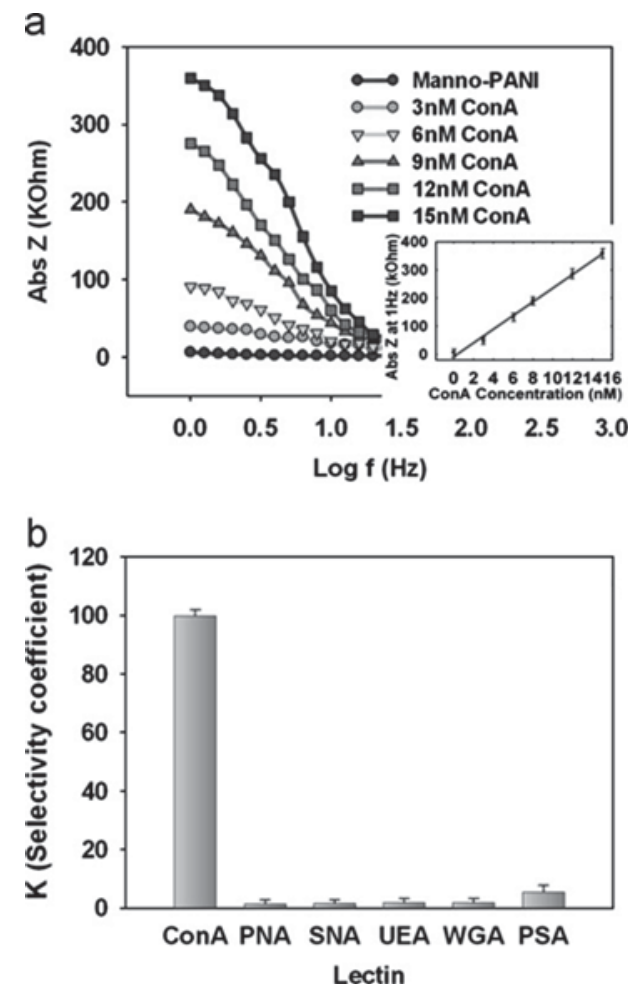

Figure 5.5. Quantification of manno-PANI and lectin interactions by electrochemical techniques for sensor application (a) EIS spectrum of manno-PANI film in the absence and presence of Con A at different concentrations Insert: Plot of impedance at 1 $\mathrm{Hz}$ vs. Con A concentration. (b) Comparison of the selectivity coefficients (K) for control lectins with respect to Con A at the same concentration (6 nM), where DZ is the difference of impedance of the manno-PANI film before and after the addition of a lectin and $\mathrm{K} 1 / 4 \mathrm{DZ}$ (control lectin)/DZ (Con A).

The formation of manno-PANI increased the substrate ITO impedance to $5000 \Omega$ at $1 \mathrm{~Hz}$. This impedance is higher than pure PANI film reported in the literature since the conjugated structure and doping mode of PANI was influenced by substituted group. When Con A was added to the manno-PANI modified ITO electrode, the resulting impedance increased remarkably. As it is reported that the peripheric protein surface adjacent to the carbohydrate recognition domain (CDR) of natural lectins may interact with hydrophobic 
groups, the increase of impedance could be attributed to the structure of polyaniline change caused by binding of $\mathrm{Con} \mathrm{A}$ to the mannose in the presence of $\mathrm{Ca}^{+2}$ ions. The $\mathrm{Con}$ A-mannose interactions result in partial deprotonation of polyaniline so that the content of imine functionality increases but quinoid form decreases. This changes the disorder in PANI, which shows higher conductivity at emeraldine form typically. As shown in Fig. 5.5a, the increase of impedance of manno-PANI modified electrode is Con A concentration-dependent in the range of $3-15 \mathrm{nM}$ with the following linear relationship.

$$
\begin{gathered}
Z_{\text {Impedance at } 1 \mathrm{~Hz}}(\mathrm{kOhm})=24.3 \times \mathrm{C}_{\mathrm{ConA}} \text { concentration }(\mathrm{nM}) \\
=8.08\left(\mathrm{r}^{2}=0: 995\right)
\end{gathered}
$$

Equation 1. Raltion between the impedence and Con A concentration

The detection limit was calculated to be $0.12 \mathrm{nM} \quad(\mathrm{S} / \sigma=3)$, where $\sigma$ is the standard deviation of the impedance obtained in the absence of Con A), which is the lowest detection limits when compared with those reported in the literature.

Additionally, in order to further ascertain the specificity of Con A and manno-PANI interactions, a panel of negative control lectins including PNA (galactose/Nacetylgalactosamine binding lectin), SNA ( $\mathrm{N}$-acetylneuraminic acid binding lectin), UEA (Fucose binding lectin), WGA (N-acetylglucosamine binding lectin), PSA (mannose binding lectin) that are known to specifically recognize other natural monosaccharide subunits were assayed in the same conditions via EIS readout (Fig. 5.5b).In stark contrast, when nonspecific lectins with the concentration of $6 \mathrm{nM}$ were added to the mannoPANI film, very small impedance changes were observed. Without specific mannoselectin interactions, the structure of manno-PANI remained intact and conductivity is a constant. The slight signal change is explained as the non-specific adsorptions of control lectins on the manno-PANI surface. These data have successfully demonstrated the feasibility of the uniquely glycosylated polyaniline biofilm containing natural saccharide for the accurate probing of specific carbohydrate- protein interactions via measurement of the conductivity changes of glycosylated aniline polymer. 
The manno-PANI modified electrode has a very good long- term stability characterized by EIS and UV-vis experiments. In Fig. D.3 and Table D.1, the impedance values at $1 \mathrm{~Hz}$ and absorbance values at $560 \mathrm{~nm}$ towards the same concentration of Con A were plotted over the measurement period of 20 weeks. The signal drift is normalized to the first measurement of sensor response on the first day. The reported values are the result of averaging at least three measurements. A maximum drift of 3\% in EIS measurement was observed in these 20 weeks highlighting the stability of the manno-PANI films for practical applications.

\subsection{Conclusion}

We have demonstrated that glycosylated aniline polymer can be used as an effective new biomaterial for label free electrochemical characterization of carbohydrate-protein interactions based on the extremely high sensitivity of manno-PANI to the local proton exchange. The deprotonation of manno-PANI caused by specific interactions between lectins and carbohydrates on the manno-PANI resulted in the switching of amine to imine in the manno-PANI supported by UV-vis absorption and XPS characterization. As a result of this conversion in manno-PANI, the conductivities of film were dramatically decreased upon binding with Con A with detec- tion limits of $1.2 \mathrm{nM}$ and a linear range of 3-15 nM. There is little signal changes in the presence of nonspecific lectins during the EIS measurement. This confirms the extreme sensitivity and selectively of manno-PANI to the specific lectin binding. The simplicity of synthesis of glycosylated aniline monomer, the versatility of poly- merization of the glycosylated aniline by chemical or electrochemi- cal oxidation, and the sensitivity of the EIS readout without using redox probes render this method feasible for various carbohydrate array fabrication and miniaturization. Thus the glycosylated aniline polymer can be used to facilitate the better understanding of the "glycomics" as well as the development of early-state disease diagnosis using carbohydrate biosensors.

\section{Acknowledgement}

Zeng X. would like to acknowledge the partly support by NIH R21, Oakland University Research Excellence funds and National Natural Science Foundation of China (No. 
21128005). Chunyan Sun likes to acknowledge the funds from China Scholarship Council for her visit at Oakland University. Authors would like to thank Professor Fen-Tair Luo to assist for Mass spectroscopic character- ization of products and Professor Qian Zhou of Mechanical Engi- neering Department to assist for XPS instrument operation. SEM instrument is funded by NSF (the award number 1040304). H. Liu would like to acknowledge the NSF support.

\section{References}

1. Bertozzi, C. R.; Kiessling; Laura, L., Chemical Glycobiology. Science 2001, 291 (5512), 2357-2364.

2. Lis, H.; Sharon, N., Lectins: Carbohydrate-Specific Proteins That Mediate Cellular Recognition†. Chem Rev 1998, 98 (2), 637-674.

3. Manning, D. D.; Bertozzi, C. R.; Pohl, N. L.; Rosen, S. D.; Kiessling, L. L., Selectin-Saccharide Interactions: Revealing Structure-Function Relationships with Chemical Synthesis. The Journal of Organic Chemistry 1995, 60 (20), 6254-6255.

4. Plante, O. J.; Palmacci, E. R.; Seeberger, P. H., Automated Solid-Phase Synthesis of Oligosaccharides. Science 2001, 291 (5508), 1523-1527.

5. Shaanan, B.; Lis, H.; Sharon, N., Structure of a legume lectin with an ordered Nlinked carbohydrate in complex with lactose. Science 1991, 254 (5033), 862-866.

6. Sharon, N.; Lis, H., Lectin biochemistry: New way of protein maturation. Nature 1986, 323 (6085), 203-204.

7. Loaiza, O. A.; Lamas-Ardisana, P. J.; Jubete, E.; Ochoteco, E.; Loinaz, I.; Cabañero, G. n.; García, I.; Penadés, S., Nanostructured Disposable Impedimetric Sensors as Tools for Specific Biomolecular Interactions: Sensitive Recognition of Concanavalin A. Analytical Chemistry 2011, 83 (8), 2987-2995.

8. Pu, K.-Y.; Shi, J.; Wang, L.; Cai, L.; Wang, G.; Liu, B., Mannose-Substituted Conjugated Polyelectrolyte and Oligomer as an Intelligent Energy Transfer Pair for 
Label-Free Visual Detection of Concanavalin A. Macromolecules 2010, 43 (23), 9690-9697.

9. Szunerits, S.; Niedziǒłka-Jönsson, J.; Boukherroub, R.; Woisel, P.; Baumann, J.-S. b.; Siriwardena, A., Label-Free Detection of Lectins on Carbohydrate-Modified Boron-Doped Diamond Surfaces. Analytical Chemistry 2010, 82 (19), 8203-8210.

10. Chen, Y.; Vedala, H.; Kotchey, G. P.; Audfray, A.; Cecioni, S.; Imberty, A.; Vidal, S.; Star, A., Electronic Detection of Lectins Using Carbohydrate-Functionalized Nanostructures: Graphene versus Carbon Nanotubes. ACS Nano 2011, 6 (1), 760770.

11. Gruber, K.; Horlacher, T.; Castelli, R.; Mader, A.; Seeberger, P. H.; Hermann, B. A., Cantilever Array Sensors Detect Specific Carbohydrate-Protein Interactions with Picomolar Sensitivity. ACS Nano 2011, 5 (5), 3670-3678.

12. Jelinek, R.; Kolusheva, S., Carbohydrate Biosensors. Chem Rev 2004, 104 (12), 5987-6016.

13. Haab, B. B., Methods and applications of antibody microarrays in cancer research. PROTEOMICS 2003, 3 (11), 2116-2122.

14. Ricard-Blum, S.; Peel, L. L.; Ruggiero, F.; Freeman, N. J., Dual polarization interferometry characterization of carbohydrate-protein interactions. Analytical Biochemistry 2006, 352 (2), 252-259.

15. Pei, Y.; Yu, H.; Pei, Z.; Theurer, M.; Ammer, C.; André, S.; Gabius, H.-J.; Yan, M.; Ramström, O., Photoderivatized Polymer Thin Films at Quartz Crystal Microbalance Surfaces: Sensors for Carbohydrate-Protein Interactions. Analytical Chemistry 2007, 79 (18), 6897-6902.

16. Shen, Z.; Huang, M.; Xiao, C.; Zhang, Y.; Zeng, X.; Wang, P. G., Nonlabeled Quartz Crystal Microbalance Biosensor for Bacterial Detection Using Carbohydrate and Lectin Recognitions. Analytical Chemistry 2007, 79 (6), 2312 2319.

17. Munoz, E. M.; Correa, J.; Fernandez-Megia, E.; Riguera, R., Probing the Relevance of Lectin Clustering for the Reliable Evaluation of Multivalent Carbohydrate 
Recognition. Journal of the American Chemical Society 2009, 131 (49), 1776517767.

18. Murthy, B. N.; Voelcker, N. H.; Jayaraman, N., Evaluation of $\alpha$-dmannopyranoside glycolipid micelles-lectin interactions by surface plasmon resonance method. Glycobiology 2006, 16 (9), 822-832.

19. Murthy, B. N.; Zeile, S.; Nambiar, M.; Nussio, M. R.; Gibson, C. T.; Shapter, J. G.; Jayaraman, N.; Voelcker, N. H., Self assembly of bivalent glycolipids on single walled carbon nanotubes and their specific molecular recognition properties. $R S C$ Advances 2012, 2 (4), 1329-1333.

20. Dai, Z.; Kawde, A.-N.; Xiang, Y.; La Belle, J. T.; Gerlach, J.; Bhavanandan, V. P.; Joshi, L.; Wang, J., Nanoparticle-Based Sensing of Glycan-Lectin Interactions. Journal of the American Chemical Society 2006, 128 (31), 10018-10019.

21. He, X.-P.; Wang, X.-W.; Jin, X.-P.; Zhou, H.; Shi, X.-X.; Chen, G.-R.; Long, Y.T., Epimeric Monosaccharide-Quinone Hybrids on Gold Electrodes toward the Electrochemical Probing of Specific Carbohydrate-Protein Recognitions. Journal of the American Chemical Society 2011, 133 (10), 3649-3657.

22. Mayer, M.; Meyer, B., Group Epitope Mapping by Saturation Transfer Difference NMR To Identify Segments of a Ligand in Direct Contact with a Protein Receptor. Journal of the American Chemical Society 2001, 123 (25), 6108-6117.

23. Doyle, M. L., Characterization of binding interactions by isothermal titration calorimetry. Current Opinion in Biotechnology 1997, 8 (1), 31-35.

24. Bogomolova, A.; Komarova, E.; Reber, K.; Gerasimov, T.; Yavuz, O.; Bhatt, S.; Aldissi, M., Challenges of Electrochemical Impedance Spectroscopy in Protein Biosensing. Analytical Chemistry 2009, 81 (10), 3944-3949.

25. Ding, L.; Cheng, W.; Wang, X.; Xue, Y.; Lei, J.; Yin, Y.; Ju, H., A label-free strategy for facile electrochemical analysis of dynamic glycan expression on living cells. Chemical Communications 2009, (46), 7161-7163.

26. Yu, X.; Xu, D.; Cheng, Q., Label-free detection methods for protein microarrays. PROTEOMICS 2006, 6 (20), 5493-5503. 
27. Zhou, Y.; Zheng, K.; Grunwaldt, J.-D.; Fox, T.; Gu, L.; Mo, X.; Chen, G.; Patzke, G. R., W/Mo-Oxide Nanomaterials: Structure-Property Relationships and Ammonia-Sensing Studies†. The Journal of Physical Chemistry C 2010, 115 (4), 1134-1142.

28. Hu, F.; Chen, S.; Wang, C.; Yuan, R.; Xiang, Y.; Wang, C., Multi-wall carbon nanotube-polyaniline biosensor based on lectin-carbohydrate affinity for ultrasensitive detection of Con A. Biosensors and Bioelectronics 2012, 34 (1), 202207.

29. Loris, R.; Hamelryck, T.; Bouckaert, J.; Wyns, L., Legume lectin structure. Biochimica et Biophysica Acta (BBA) - Protein Structure and Molecular Enzymology 1998, 1383 (1), 9-36.

30. Ray, A.; Asturias, G. E.; Kershner, D. L.; Richter, A. F.; MacDiarmid, A. G.; Epstein, A. J., Polyaniline: Doping, structure and derivatives. Synthetic Metals 1989, 29 (1), 141-150.

31. Ray, A.; Richter, A. F.; MacDiarmid, A. G.; Epstein, A. J., Polyaniline: protonation/deprotonation of amine and imine sites. Synthetic Metals 1989, 29 (1), 151-156.

32. Chan, H. S. O.; Ng, S. C.; Ho, P. K. H., Polyanilines Doped with Phosphonic Acids: Their Preparation and Characterization. Macromolecules 1994, 27 (8), 2159-2164.

33. Dung Nguyen, T.; Camalet, J. L.; Lacroix, J. C.; Aeiyach, S.; Pham, M. C.; Lacaze, P. C., Polyaniline electrodeposition from neutral aqueous media: Application to the deposition on oxidizable metals. Synthetic Metals 1999, 102 (1-3), 1388-1389.

34. Solanki, P. R.; Prabhakar, N.; Pandey, M. K.; Malhotra, B. D., Nucleic acid sensor for insecticide detection. Journal of Molecular Recognition 2008, 21 (4), 217-223.

35. Yue, J.; Epstein, A. J., XPS study of self-doped conducting polyaniline and parent systems. Macromolecules 1991, 24 (15), 4441-4445.

36. Yue, J.; Wang, Z. H.; Cromack, K. R.; Epstein, A. J.; MacDiarmid, A. G., Effect of sulfonic acid group on polyaniline backbone. Journal of the American Chemical Society 1991, 113 (7), 2665-2671. 
37. Zhou, H.; Lin, Y.; Yu, P.; Su, L.; Mao, L., Doping polyaniline with pristine carbon nanotubes into electroactive nanocomposite in neutral and alkaline media. Electrochemistry Communications 2009, 11 (5), 965-968.

38. Wei, Y.; Focke, W. W.; Wnek, G. E.; Ray, A.; MacDiarmid, A. G., Synthesis and electrochemistry of alkyl ring-substituted polyanilines. The Journal of Physical Chemistry 1989, 93 (1), 495-499.

39. Kilmartin, P. A.; Wright, G. A., Photoelectrochemistry and spectroscopy of substituted polyanilines. Synthetic Metals 1999, 104 (3), 145-156.

40. Snauwaert, P.; Lazzaroni, R.; Riga, J.; Verbist, J. J., Electronic structure of polyaniline and substituted derivatives. Synthetic Metals 1987, 18 (1-3), 335-340.

41. Duić, L.; Mandić, Z., Counter-ion and pH effect on the electrochemical synthesis of polyaniline. Journal of Electroanalytical Chemistry 1992, 335 (1-2), 207-221.

42. Granot, E.; Katz, E.; Basnar, B.; Willner, I., Enhanced Bioelectrocatalysis Using Au-Nanoparticle/Polyaniline Hybrid Systems in Thin Films and Microstructured Rods Assembled on Electrodes. Chemistry of Materials 2005, 17 (18), 4600-4609.

43. Yin, X.; Ding, J.; Zhang, S.; Kong, J., Enantioselective sensing of chiral amino acids by potentiometric sensors based on optical active polyaniline films. Biosensors and Bioelectronics 2006, 21 (11), 2184-2187.

44. Ervin, E. N.; White, R. J.; White, H. S., Sensitivity and Signal Complexity as a Function of the Number of Ion Channels in a Stochastic Sensor. Analytical Chemistry 2008, 81 (2), 533-537.

45. Lathrop, D. K.; Ervin, E. N.; Barrall, G. A.; Keehan, M. G.; Kawano, R.; Krupka, M. A.; White, H. S.; Hibbs, A. H., Monitoring the Escape of DNA from a Nanopore Using an Alternating Current Signal. Journal of the American Chemical Society 2010, 132 (6), 1878-1885.

46. White, R. J.; Ervin, E. N.; Yang, T.; Chen, X.; Daniel, S.; Cremer, P. S.; White, H. S., Single Ion-Channel Recordings Using Glass Nanopore Membranes. Journal of the American Chemical Society 2007, 129 (38), 11766-11775. 
47. Sazou, D., Electrodeposition of ring-substituted polyanilines on Fe surfaces from aqueous oxalic acid solutions and corrosion protection of Fe. Synthetic Metals 2001, 118 (1-3), 133-147.

48. Yavuz, A. G.; Uygun, A.; Bhethanabotla, V. R., Preparation of substituted polyaniline/chitosan composites by in situ electropolymerization and their application to glucose sensing. Carbohydrate Polymers 2010, 81 (3), 712-719.

49. Lis, H.; Sharon, N., Lectins as Molecules and as Tools. Annual Review of Biochemistry 1986, 55 (1), 35-67.

50. Otto, K.; Elwing, H.; Hermansson, M., Effect of Ionic Strength on Initial Interactions ofEscherichia coli with Surfaces, Studied On-Line by a Novel Quartz Crystal Microbalance Technique. Journal of Bacteriology 1999, 181 (17), 52105218.

51. Masters, J. G.; Sun, Y.; MacDiarmid, A. G.; Epstein, A. J., Polyaniline: Allowed oxidation states. Synthetic Metals 1991, 41 (1-2), 715-718.

52. Albuquerque, J. E.; Mattoso, L. H. C.; Balogh, D. T.; Faria, R. M.; Masters, J. G.; MacDiarmid, A. G., A simple method to estimate the oxidation state of polyanilines. Synthetic Metals 2000, 113 (1-2), 19-22.

53. Ayad, M. M.; Salahuddin, N. A.; Alghaysh, M. O.; Issa, R. M., Phosphoric acid and $\mathrm{pH}$ sensors based on polyaniline films. Current Applied Physics 2010, 10 (1), 235-240.

54. Lindfors, T.; Ivaska, A., Potentiometric and UV-vis characterisation of Nsubstituted polyanilines. Journal of Electroanalytical Chemistry 2002, 535 (1-2), $65-74$.

55. Libert, J.; Cornil, J.; dos Santos, D. A.; Brédas, J. L., From neutral oligoanilines to polyanilines: A theoretical investigation of the chain-length dependence of the electronic and optical properties. Physical Review B 1997, 56 (14), 8638-8650.

56. Zand, R.; Agrawal, B. B. L.; Goldstein, I. J., pH-Dependent Conformational Changes of Concanavalin A. Proceedings of the National Academy of Sciences 1971, 68 (9), 2173-2176. 
57. Appenzeller-Herzog, C.; Roche, A.-C.; Nufer, O.; Hauri, H.-P., pH-induced Conversion of the Transport Lectin ERGIC-53 Triggers Glycoprotein Release. Journal of Biological Chemistry 2004, 279 (13), 12943-12950.

58. Iobst, S. T.; Wormald, M. R.; Weis, W. I.; Dwek, R. A.; Drickamer, K., Binding of sugar ligands to $\mathrm{Ca}(2+)$-dependent animal lectins. I. Analysis of mannose binding by site-directed mutagenesis and NMR. Journal of Biological Chemistry 1994, 269 (22), 15505-15511.

59. Focke, W. W.; Wnek, G. E.; Wei, Y., Influence of oxidation state, pH, and counterion on the conductivity of polyaniline. The Journal of Physical Chemistry 1987, 91 (22), 5813-5818.

60. Macdiarmid, A. G.; Chiang, J. C.; Richter, A. F.; Epstein, A. J., Polyaniline: a new concept in conducting polymers. Synthetic Metals 1987, 18 (1-3), 285-290.

61. Lim, S. L.; Tan, K. L.; Kang, E. T., In Situ XPS Study of the Interactions of Evaporated Copper Atoms with Neutral and Protonated Polyaniline Films. Langmuir 1998, 14 (18), 5305-5313.

62. Tan, K. L.; Tan, B. T. G.; Kang, E. T.; Neoh, K. G., X-ray photoelectron spectroscopy studies of the chemical structure of polyaniline. Physical Review B 1989, 39 (11), 8070-8073.

63. Choudary, B. M.; Roy, M.; Roy, S.; Kantam, M. L.; Sreedhar, B.; Kumar, K. V., Preparation, Characterization and Catalytic Properties of Polyaniline-Supported Metal Complexes. Advanced Synthesis \& Catalysis 2006, 348 (12-13), 1734-1742.

64. van der Sanden, M. C. M., Counter-ion induced processibility of conducting polymers: 1. Acid-assisted oxidative doping and solubilization. Synthetic Metals 1997, 87 (2), 137-140. 


\section{Chapter 6 : Ferrocene-Mannose Conjugate: A Potential Electrochemical Probe for Carbohydrate-Lectin Interactions ${ }^{5}$}

Giri Vegesna, ${ }^{\mathrm{a}}$ Fen-Tair Luo ${ }^{\mathrm{b}}$, and Haiying Liu ${ }^{\mathrm{a} *}$

aDepartment of Chemistry, Michigan Technological University, Houghton, MI 49931

${ }^{b}$ Institute of Chemistry, Academia Sinica, Taipei, Taiwan 11529, Republic of China

${ }^{5}$ This chapter may be submitted to a journal for possible publication in the near future. 


\subsection{Abstract}

Synthesis of two ferrocene-mannose conjugates $\mathbf{A}, \mathbf{B}$, with two different linkers with thiol end group is reported. The ferrocene-mannose conjugate was prepared by the reaction of aliphatic amine incorporated acylated $\beta$-D-mannopyranosyl residue with ferrocenyl acid chloride. This resultant unsymmetrical ferrocene mannose conjugate is used to functionlize other cyclopentadienyl ring with thiol end group. This thiol end group is used to anchor the ferrocene-mannose conjugate to the electrode surface to use this as a potential electrochemical probe to study carbohydrate-protein interactions. Versatility of this synthesis involves the attachment of two different groups, a carbohydrate reporter and thiol anchoring group, on the two cyclopentadienyl rings of the ferrocene. This molecule can be used in the development of potential electrochemical probe with high sensitivity to study the carbohydrate-protein interactions.

\subsection{Introduction}

Study the interactions between the carbohydrates and cell surface proteins is crucial in understanding many biological processes. These biological processes include viral and bacterial infections, cell recognition and adhesion, immune response, fertilization, and cancer metastasis. Generally used techniques to study the carbohydrate-protein interactions include fluoresecence spectroscopy, calorimetry, surface Plasmon resonance, quartz crystal micro balance, and electrochemical methods. Among these techniques electrochemical biosesning has more viable in terms of cost, sensitivity, ease of operation and opportunities for miniaturization. The highly favorable electrochemical and spectroscopic properties of ferrocene and its derivatives makes them a valuable tool for the construction of molecular systems such as sensors. The high stability of ferrocene group in aqueous and aerobic media is an additional advantage. Several synthetic strategies for ferrocene, carbohydrate conjugation is reported in literature. Only very few cases of cases of carbohydrate coupled redox-active moieties are reported to study the carbohydrateprotein interactions. This method involves the measurement of change in electrical property of the ferrocene triggered by the carbohydrate binding event. But, the weak 
carbohydrate-protein interactions generates a very weak electrical signal by monovalent binding event. Synthetic multivalent carbohydrate binding is the better approach to enhance the sensitivity. This can be achieved by anchoring the ferrocene-carbohydrate conjugate on any metal or electrode surface. For this purpose an unsymmetrical ferrocene derivative is to be prepared by functionalizing the one cyclopentdienyl ring with carbohydrate receptor and the second one with metal anchoring group such as thiol.

\subsection{Experimental Section}

\subsubsection{Instrumentation}

${ }^{1} \mathrm{H}$ NMR and ${ }^{13} \mathrm{C}$ NMR spectra were obtained by using a $400 \mathrm{MHz}$ NMR spectrophotometer instrument. ${ }^{1} \mathrm{H}$ and ${ }^{13} \mathrm{C}$ NMR spectra were recorded in $\mathrm{CDCl}_{3}$, chemical shifts $(\delta)$ are given in ppm relative to solvent peaks $\left({ }^{1} \mathrm{H}: \delta 7.26 ;{ }^{13} \mathrm{C}: \delta 77.3\right)$ as internal standard. HRMS were measured with fast atom bombardment (FAB) ionization or matrixassisted laser desorption ionization (MALDI) using a double focusing magnetic or a matrix assisted laser desorption-time of flight mass spectrometer. Infrared (IR) samples were prepared as $\mathrm{KBr}$ pellets, and spectra were obtained in the $400-4000 \mathrm{~cm}^{-1}$ range using a Bruker Tensor-27 FTIR spectrometer.

\subsubsection{Materials}

Unless otherwise indicated, all reagents and solvents were obtained from commercial suppliers and used without further purification. Air- and moisture-sensitive reactions were conducted in oven-dried glassware using a standard Schlenk line or drybox techniques under an inert atmosphere of dry nitrogen. 


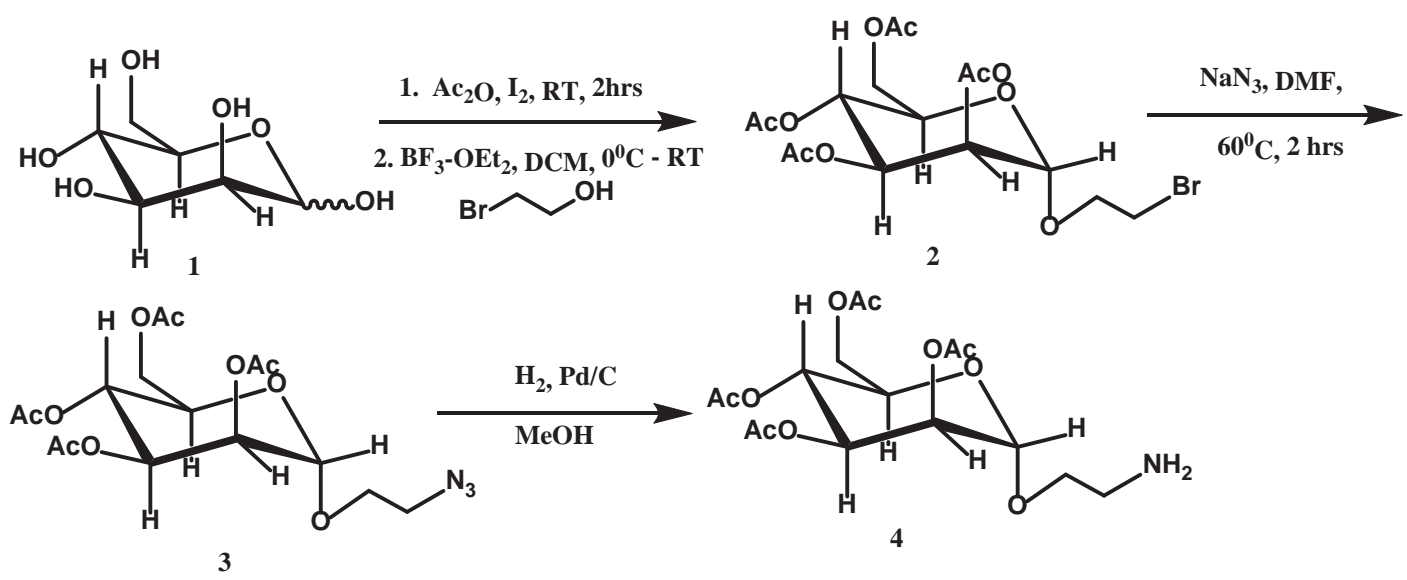

Scheme 6.1. Synthetic route to Compound 4

Synthesis of Compound 2: To the stirred suspension of D- mannose 1 (5 gm, $0.027 \mathrm{~mol})$, in acetic anhydride (50 ml), iodine $(250 \mathrm{mg}$ ) was added. Reaction mass began to warm is sign for the initiation of acetylation and sugar started dissolving in the solution. Reaction run at room temperature for 30 minutes. After checking the TLC, iodine was quenched with aqueous sodium thiosulfate solution, pentaacetate compound was extracted into DCM $(2 \times 60 \mathrm{ml})$ and washed with aqueous sodium carbonate solution until $\mathrm{pH}$ of the aqueous layer is neutral. Finally wash the organic layer with brine solution and dry with sodium sulphate was concentrated under reduced pressure to get syrup (10gm, 92\%). Crude product has been used as such for the next step. Acylated mannose (5 gm, $0.012 \mathrm{~mol}$ ) and bromoethanol $(1.09 \mathrm{ml}, 0.015 \mathrm{~mol})$ were dissolved in dry DCM $(40 \mathrm{ml})$ under nitrogen atmosphere. Then $\mathrm{BF}_{3}-\mathrm{OEt}_{2}(8.4 \mathrm{ml}, 0.066 \mathrm{~mol})$ was added dropwise using addition funnel at $0^{\circ} \mathrm{C}$ and reaction mass was allowed to come to room temperature and stir it for 12 hours. After checking the TLC, cool the reaction mass to $0^{\circ} \mathrm{C}$ and slowly quench it with cold aqueous $\mathrm{NaHCO}_{3}$ solution and let it stir for 10 minutes. Later organic layer was diluted with DCM $(30 \mathrm{ml})$ and washed with aqueous $\mathrm{NaHCO}_{3}$ solutionfollowed by with brine. Organic layer was dried with $\mathrm{Na}_{2} \mathrm{SO}_{4}$ and concentrated under reduced pressure to yield a light brown oily compound. Recrystallization in $\mathrm{Et}_{2} \mathrm{O}: H e x a n e(1: 5)$ gave white crystal compound (3.5 gm, 60\%). ${ }^{1} \mathrm{H}$ NMR (400 MHz, $\left.\mathrm{CDCl}_{3}\right)$ : $85.34-5.23(\mathrm{~m}, 3 \mathrm{H}), 4.85(\mathrm{~s}, 1 \mathrm{H})$, 4.27-4.22 (m, 1H), 4.15-4.08 (m, 2H), 3.98-3.83 (m, 2H), 3.51-3.47 (t, $j=8.0 \mathrm{~Hz}, 2 \mathrm{H}), 2.13$ 
(s, 3H) 2.08 (s, 3H), 2.03 (s, 3H), 1.97 (s, 3H). ${ }^{13} \mathrm{C}$ NMR (100 MHz, $\left.\mathrm{CDCl}_{3}\right): \delta 170.80$, $170.21,170.05,169.95,97.95,69.63,69.23,69.14,68.69,66.22,62.62,29.80,21.06$, 20.94, 20.90, 20.86.

Synthesis of Compound 3: Compound 2 (5 gm, $10.9 \mathrm{mmol}$ ) and sodium azide (4.2 gm, $65.4 \mathrm{mmol})$ were dissolved in dry DMF $(100 \mathrm{~mL})$ and the reaction mass was stirred at $60^{\circ}$ $\mathrm{C}$ for $3 \mathrm{hrs}$. Cool the reaction mass for room temperature, remove the solvent under reduce pressure. Dilute the compound with DCM $(100 \mathrm{~mL})$, wash the organic layer with water and brine solution. Dry the organic layer over sodium sulfate, evaporate the solvent under reduced pressure. The crude compound was purified by column chromatography using ETOAc/Hexane (30/70) to yield a syrupy oil (3.6 gm, 80\%). ${ }^{1} \mathrm{H}$ NMR (400 $\left.\mathrm{MHz}, \mathrm{CDCl}_{3}\right)$ : 85.38-5.23 (m, 3H), 4.88-4.82 (m, 1H), 4.31-4.23 (m, 1H), 4.14-4.00 (m, 2H), 3.89-3.81 $(\mathrm{m}, 1 \mathrm{H}), 3.69-3.62(\mathrm{~m}, 1 \mathrm{H}), 3.52-3.38(\mathrm{~m}, 2 \mathrm{H}), 2.14(\mathrm{~s}, 3 \mathrm{H}), 2.08(\mathrm{~s}, 3 \mathrm{H}), 2.03(\mathrm{~s}, 3 \mathrm{H})$, 1.97 (s, 3H). ${ }^{13} \mathrm{C} \mathrm{NMR}\left(100 \mathrm{MHz}, \mathrm{CDCl}_{3}\right): \delta 170.80,170.20,169.99,169.95,97.95,69.59$, $69.05,67.24,66.21,62.66,50.56,21.06,20.93,20.90,20.85$.

Synthesis of Compound 4: Compound 3 (2 gm, $4.78 \mathrm{mmol}$ ) was dissolved in methanol (40 $\mathrm{mL}), 10 \% \mathrm{Pd} / \mathrm{C}(0.2 \mathrm{gm})$ was added and acidify the reaction mass with drop of acetic acid. Stir the reaction mass for $2 \mathrm{hrs}$ at room temperature under hydrogen gas balloon. Reaction mixture was filtered through celite and solvent was concentrated under reduced pressure to afford the corresponding amine (1.59 gm, 85\%). This compound was directly used in the next step without further purification. 

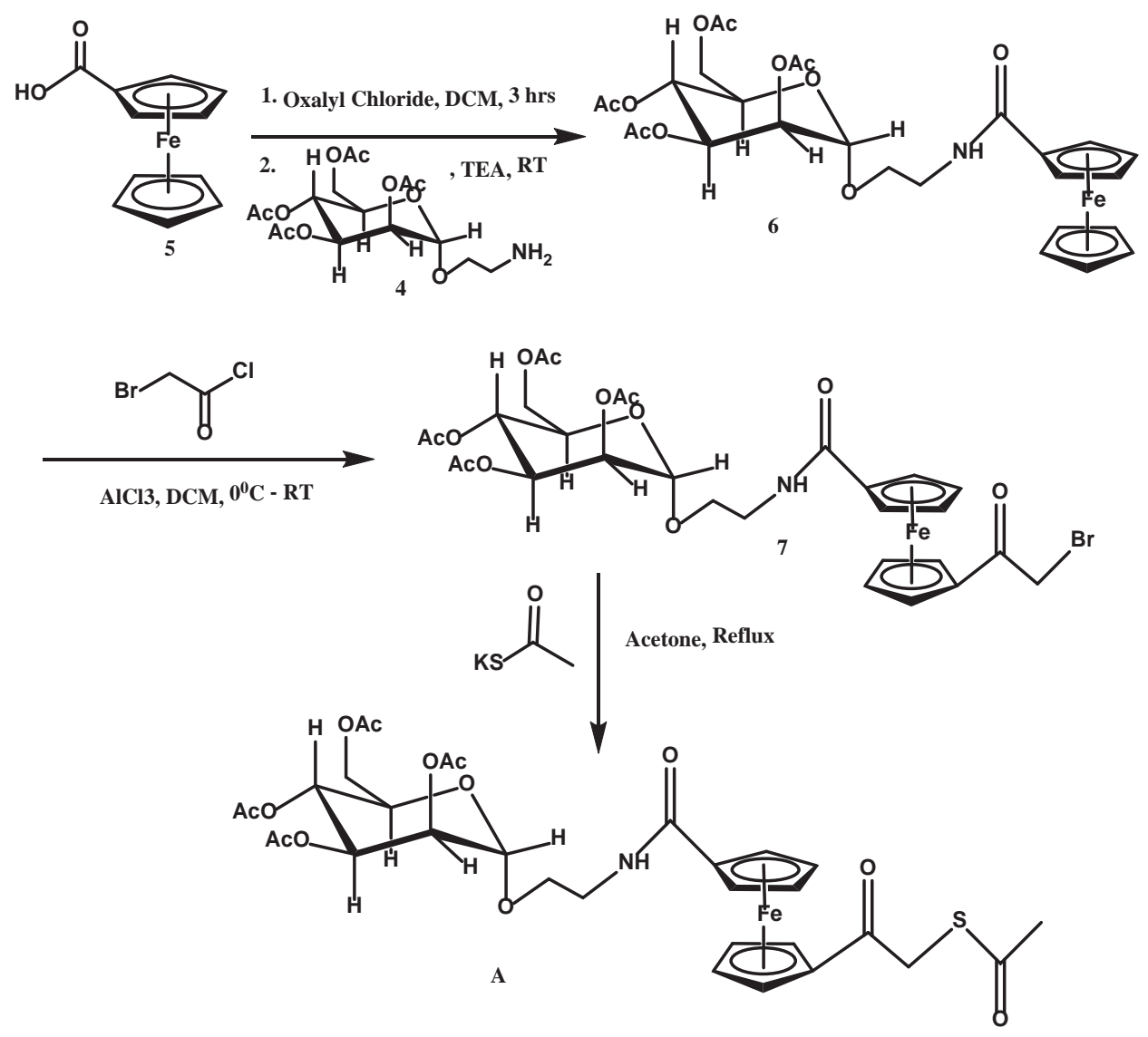

Scheme 6.2. Synthetic route to Compound A

Synthesis of Compound 6: The compound 5 (2 gm, $8.69 \mathrm{mmol})$ was dissolved in dry DCM $(50 \mathrm{~mL})$ in a $100 \mathrm{~mL}$ round bottom flask under nitrogen atmosphere. Oxalyl chloride (4.4 gm, $34.76 \mathrm{mmol}$ ) was slowly added at $0^{\circ} \mathrm{C}$ using syringe. Reaction mass was heated to $40^{0}$ $\mathrm{C}$ for $4 \mathrm{hrs}$ under inert atmosphere. Cool the reaction mass, distill off the solvent and the excess oxalylchloride under nitrogen atmosphere. Then, to the stirred solution of compound 4 (3.4 gm, 8.69) and triethyl amine (2.1 gm, $21.75 \mathrm{mmol})$ in dry DCM (100 mL) at room temperature, crude ferrocene acid chloride diluted in dry DCM $(50 \mathrm{~mL})$ was added under nitrogen atmosphere. After the reaction mas was stirred overnight at room temperature, the solvent was distilled off under reduced pressure. Crude residue was purified by column chromatography using EtOAc/DCM (50/50) to yield the compound $\mathbf{6}$ (2.6 gm, 50\%). ${ }^{1} \mathrm{H}$ NMR (400 MHz, $\left.\mathrm{CDCl}_{3}\right): \delta 6.16-6.10(\mathrm{~m}, 1 \mathrm{H}), \quad 5.36-5.33(\mathrm{~m}, 1 \mathrm{H})$, 
5.27-5.22 (m, 2H), 4.86-4.83 (m, 1H), 4.67 (brs, 2H), 4.33 (brs, $2 \mathrm{H}), 4.25-4.18(\mathrm{~m}, 6 \mathrm{H})$, 4.10-4.07 (m, 1H), 4.02-3.96 (m, 1H), 3.87-3.80 (m, 1H), 3.65-3.59 (m, 2H), $2.15(\mathrm{~s}, 1 \mathrm{H})$, $2.12(\mathrm{~s}, 3 \mathrm{H}), 2.05$ (s, 3H), $2.01(\mathrm{~s}, 3 \mathrm{H}), 1.98(\mathrm{~s}, 3 \mathrm{H}) .{ }^{13} \mathrm{C}$ NMR $\left(100 \mathrm{MHz}, \mathrm{CDCl}_{3}\right): \delta$ $170.78,170.64,170.23,170.19,169.84,97.98,75.97,70.76,69.99,69.60,69.20,69.04$, $68.39,68.06,66.32,62.72,54.02,39.35,29.48,21.05,20.92,20.90,20.89$. HRMS (FAB) calcd for $\mathrm{C}_{29} \mathrm{H}_{39} \mathrm{FeNO}_{11}[\mathrm{M}]^{+}, 603.4650$; found, 603.0000 .

Synthesis of Compound 7: To the stirred suspension of $\mathrm{AlCl}_{3}(2.4 \mathrm{gm} 18.4 \mathrm{mmol})$ in dry DCM $(80 \mathrm{~mL})$, Bromo acetyl chloride $(1.81 \mathrm{gm}, 11.5 \mathrm{mmol})$ was added at room temperature under nitrogen atmosphere and stir this suspension for 1 hour until all $\mathrm{AlCl}_{3}$ was dissolved in solution. Then compound 6 (2.7 gm, $4.6 \mathrm{mmol})$ was added drop wise at $0^{\circ} \mathrm{C}$ using addition funnel for 1 hour. As soon as addition completed, quench the reaction mass by adding ice cold water. Product was extracted into DCM $(2 \times 100 \mathrm{ml})$ and organic layer was washed with aqueous sodium bicarbonate and brine solution respectively. Dry the organic layer with Sodium sulfate and concentrated under vacuum. Compound was purified by column chromatography using EtOAc/hexane (50/50) to get the orange syrup (1.6 gm, 50\%). ${ }^{1} \mathrm{H}$ NMR (400 MHz, $\left.\mathrm{CDCl}_{3}\right): \delta 6.31$ (brs, $\left.1 \mathrm{H}\right), 5.26-5.23(\mathrm{~m}, 1 \mathrm{H}), 5.18-$ $5.16(\mathrm{~m}, 2 \mathrm{H}), 4.80-4.72(\mathrm{~m}, 2 \mathrm{H}), 4.61$ (brs, $2 \mathrm{H}), 4.54$ (brs, $1 \mathrm{H}), 4.23-4.09(\mathrm{~m}, 7 \mathrm{H}), 4.01$ $3.98(\mathrm{~m}, 1 \mathrm{H}), 3.93-3.88(\mathrm{~m}, 1 \mathrm{H}), 3.76-3.74(\mathrm{~m}, 1 \mathrm{H}), 3.55-3.49(\mathrm{~m}, 2 \mathrm{H}), 2.03$ (brs, $3 \mathrm{H})$, 1.98-1.97 (m, 3H), 1.92 (brs, 3H), 1.89-1.87 (m, 3H). $\left.{ }^{13} \mathrm{C} \mathrm{NMR} \mathrm{(100} \mathrm{MHz,} \mathrm{CDCl}_{3}\right): \delta$ $195.98,170.78,170.74,170.19,169.83,97.92,74.24,72.06,71.80,70.59,70.06,69.58$, 69.25, 69.00, 68.47, 67.98, 67.61, 66.31, 66.09, 62.70, 39.40, 33.04, 32.36, 21.03, 20.89. HRMS (FAB) calcd for $\mathrm{C}_{31} \mathrm{H}_{40} \mathrm{BrFeNNaO}_{12}[\mathrm{M}+\mathrm{Na}]^{+}$, 747.3978; found, 746.9000 .

Synthesis of Compound $\mathbf{A}$ : Compound $7(0.2 \mathrm{gm}, 0.27 \mathrm{mmol})$ and potassium thio acetate ( $0.03 \mathrm{gm}, 0.32 \mathrm{mmol})$ were dissolved in actone $(20 \mathrm{~mL})$ and this reaction mixture was refluxed for $5 \mathrm{hrs}$. Acetone was removed under reduced pressed and the crude syrup was adsorbed on silica gel. Compound was purified on column chromatography using hexane/EtOAc/acetone (4/5/1) to afford the compound 8 (0.09 gm, 45\%). ${ }^{1} \mathrm{H}$ NMR (400 $\left.\mathrm{MHz}, \mathrm{CDCl}_{3}\right): \delta 6.57$ (brs, $\left.1 \mathrm{H}\right), \quad 5.37-5.33(\mathrm{~m}, 1 \mathrm{H}), 5.29-5.25(\mathrm{~m}, 2 \mathrm{H}), 4.92$ (brs, $\left.1 \mathrm{H}\right)$, 4.81-4.76 (m, 4H), 4.60 (brs, 2H), 4.42 (brs, 2H), 4.26-4.22 (m, 1H), 4.14 (s, 2H), 4.10- 
$4.03(\mathrm{~m}, 2 \mathrm{H}), 3.87-3.84(\mathrm{~m}, 1 \mathrm{H}), 3.68-3.60(\mathrm{~m}, 2 \mathrm{H}), 2.44(\mathrm{~s}, 3 \mathrm{H}), 2.15(\mathrm{~s}, 3 \mathrm{H}), 2.07(\mathrm{~s}$, 3H), 2.02 (s, 3H), 1.97 (s, 3H). ${ }^{13} \mathrm{C}$ NMR (100 MHz, $\left.\mathrm{CDCl}_{3}\right): \delta$ 196.73, 195.85, 170.84, $170.22,170.08,169.88,97.83,78.48,74.04,73.98,72.04,71.67,71.55,70.65,69.66$, $69.28,68.93,67.42,66.36,62.70,39.38,37.73,30.55,21.09,20.95,20.91$. HRMS (FAB) calcd for $\mathrm{C}_{33} \mathrm{H}_{43} \mathrm{FeNO}_{13} \mathrm{~S}[\mathrm{M}]^{+}, 719.6034$; found, 719.0000.

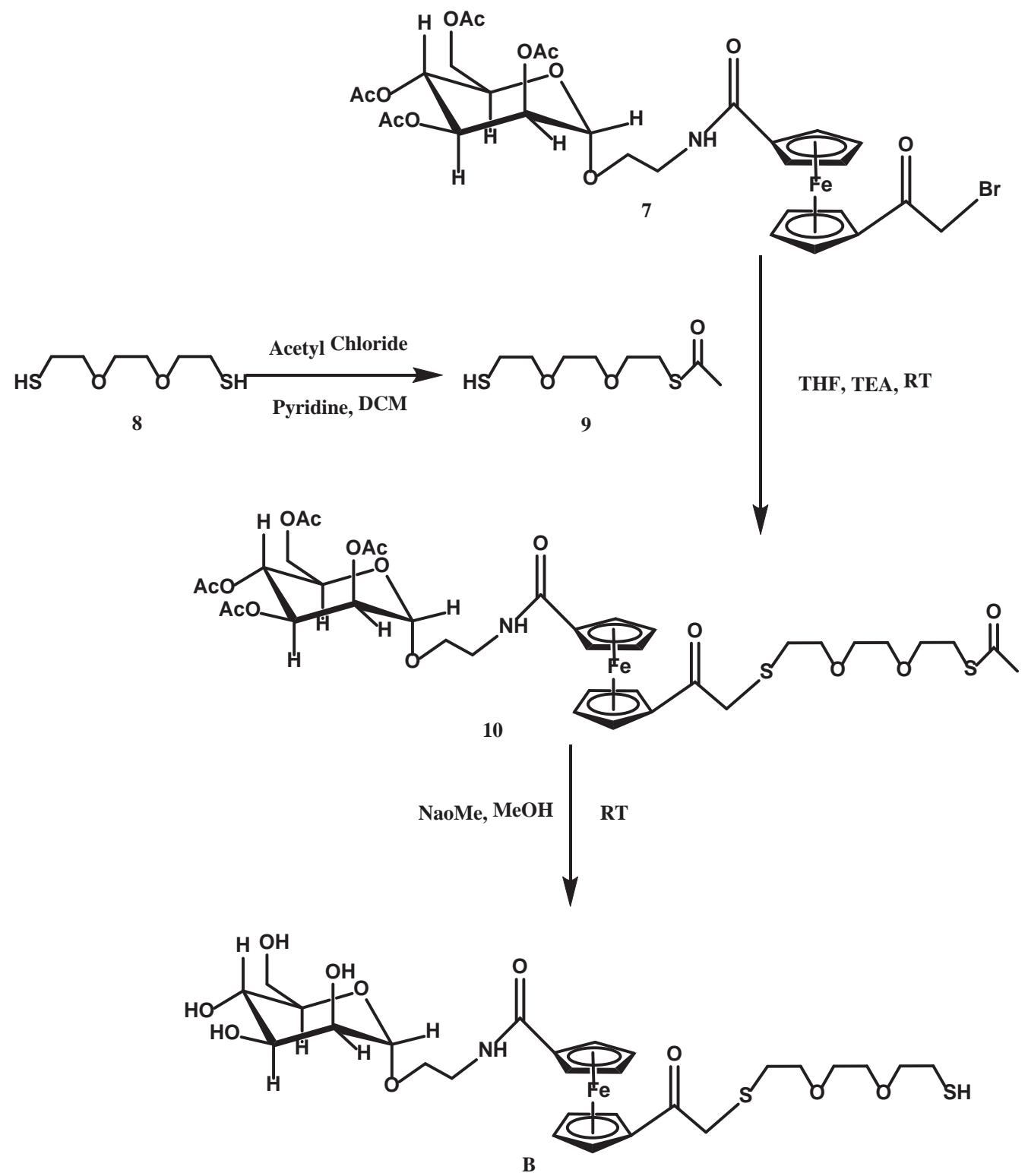

Scheme 6.3. Synthetic route to Compound B 
Synthesis of Compound 9: To the stirred solution of compound $\mathbf{8}(3 \mathrm{gm}, 16.4 \mathrm{mmol})$ in dry DCM (70 mL), pyrididne (1.2 gm, $16.4 \mathrm{mmol})$ was added. Acetyl chloride (1.4 gm, 18.2 mmol) was added drop wise through addition funnel to this reaction mixture. Stir the reaction mass at room temperature for 6 hrs. Wash the organic layer with water and brine solution, dry the organic layer over sodium sulfate. The crude compound was purified with column chromatography using EtOAc/DCM (30/70) to afford the compound 9 (2.2 gm, 60\%). ${ }^{1} \mathrm{H}$ NMR (400 MHz, $\left.\mathrm{CDCl}_{3}\right): \delta 3.59-3.53(\mathrm{~m}, 8 \mathrm{H}), 3.06-3.02(\mathrm{~m}, 2 \mathrm{H}), 2.67-2.63(\mathrm{~m}$, 2H), 2.29 (s, 3H), 1.56-1.52 (t, $\left.J=8.0 \mathrm{~Hz}, 1 \mathrm{H}) .{ }^{13} \mathrm{C} \mathrm{NMR} \mathrm{(100} \mathrm{MHz,} \mathrm{CDCl}_{3}\right): \delta 195.62$, 73.10, 70.43, 70.36, 70.34, 69.97, 30.76, 29.03, 24.47. HRMS (FAB) calcd for $\mathrm{C}_{8} \mathrm{H}_{17} \mathrm{O}_{3} \mathrm{~S}_{2}$ $[\mathrm{M}]^{+}, 225.0619$; found, 225.0616.

Synthesis of Compound 10: To the stirred solution of compound 7 and compound 9 ( 0.3 gm, $0.41 \mathrm{mmol})$ in Dry THF $(30 \mathrm{~mL})$, triethyl amine (0.04 gm, $0.49 \mathrm{mmol})$ was added. The resultant reaction mixture was stirred at room temperature for $5 \mathrm{hrs}$. Solvent was removed under reduced pressure and the crude compound was purified by column chromatography using Hexane/EtOAc/Acetone (3/6/1) to afford the compound 10 (0.2 gm, 60\%). ${ }^{1} \mathrm{H}$ NMR (400 MHz, $\left.\mathrm{CDCl}_{3}\right): \delta 6.35$ (brs, 1H), 5.37-5.34 (m, 1H), 5.29-5.24 (m, 2H), 4.92 (brs, $\left.1 \mathrm{H}\right)$, 4.76 (brs, $2 \mathrm{H}), 4.66$ (brs, $2 \mathrm{H}), 4.56$ (brs, $2 \mathrm{H}), 4.37$ (brs, $2 \mathrm{H}), 4.27-4.23$ (m, 1H), 4.12-4.02 $(\mathrm{m}, 2 \mathrm{H}), 3.90-3.86(\mathrm{~m}, 1 \mathrm{H}), 3.70-3.58(\mathrm{~m}, 12 \mathrm{H}), 3.11-3.00(\mathrm{~m}, 2 \mathrm{H}), 2.85-2.79(\mathrm{~m}, 2 \mathrm{H})$, 2.34-2.25 (m, 3H), 2.13 (s, 3H), 2.06 (s, 3H), 2.01 (s, 3H), 1.97 (s, 3H). ${ }^{13} \mathrm{C}$ NMR (100 $\left.\mathrm{MHz}, \mathrm{CDCl}_{3}\right): \delta 195.72,170.83,170.22,170.14,169.88,97.93,94.66,74.00,71.99,71.86$, 70.52, 70.47, 69.87, 69.63, 69.30, 68.99, 67.67, 66.35, 62.75, 39.39, 39.13, 38.63, 30.79, 29.04, 21.10, 20.93. HRMS (FAB) calcd for $\mathrm{C}_{39} \mathrm{H}_{55} \mathrm{FeNO}_{15} \mathrm{~S}_{2}[\mathrm{M}]^{+}, 867.8267$; found, 867.0000 .

Synthesis of Compound B: To the stirred solution of compound $\mathbf{1 0}(0.2 \mathrm{gm}, 0.23 \mathrm{mmol})$ in $\mathrm{MeOH}(20 \mathrm{~mL}), \mathrm{NaOMe}(0.012 \mathrm{gm}, 0.23 \mathrm{mmol})$ was added at room temperature under nitrogen atmosphere. The resultant reaction mixture was stirred at room temperature for 4 hrs. Amberlyte $15\left(\mathrm{H}^{+}\right)$was added until the solution become neutral and the resulting reaction mixture was filtered. Solvent was removed under reduced pressure and crude 
compound was dissolved in water and washed with ether. Aqueous layer was concentrated under reduced pressure to yield the product. ${ }^{1} \mathrm{H}$ NMR (400 MHz, $\mathrm{CD}_{3} \mathrm{OD}$ ): $\delta 6.35$ (brs, 1H), 5.37-5.34 (m, 1H), 5.29-5.24 (m, 2H), 4.92 (brs, 1H), 4.76 (brs, 2H), 4.66 (brs, 2H), 4.56 (brs, $2 \mathrm{H}), 4.37$ (brs, $2 \mathrm{H}), 4.27-4.23(\mathrm{~m}, 1 \mathrm{H}), 4.12-4.02(\mathrm{~m}, 2 \mathrm{H}), 3.90-3.86(\mathrm{~m}, 1 \mathrm{H})$, 3.70-3.58 (m, 12H), 3.11-3.00 (m, 2H), 2.85-2.79 (m, 2H). $\left.{ }^{13} \mathrm{C} \mathrm{NMR} \mathrm{(100} \mathrm{MHz,} \mathrm{CDCl}_{3}\right)$ : $\delta$ 195.72, 170.83, 170.22, 170.14, 169.88, 97.93, 94.66, 74.00, 71.99, 71.86, 70.52, 70.47, $69.87,69.63,69.30,68.99,67.67,66.35,62.75$.

\subsection{Conclusion}

We successfully prepared two unsymmetrical ferrocene-mannose conjugates A, B with two different linkers having a thiol end group. This thiol end group is to attach this conjugate molecule on a metal surface. This binding generates the synthetic multivalent binding interactions of the mannose with analyte. So this molecule can be used to construct potential carbohydrate bio sensor to study the carbohydrate-protein interactions with high sensitivity. 


\section{APPENDIX A \\ Supporting information for chapter 2}

\section{Highly Water-soluble BODIPY-based Fluorescent Probe for Sensitive and Selective Detection of Nitric Oxide in Living Cells}

Giri Vegesna, ${ }^{a}$ Srinivas Sripathi, ${ }^{b}$ Jingtuo Zhang, ${ }^{a}$ Shilei Zhu, ${ }^{a}$ Weilue He, ${ }^{c}$ Fen-Tair Luo, ${ }^{\mathrm{d}}$ Wan Jin Jahng, ${ }^{\mathrm{b}}$ Megan Frost, ${ }^{\mathrm{c}}$ and Haiying Liu ${ }^{\mathrm{a} *}$

${ }^{a}$ Department of Chemistry, ${ }^{b}$ Department of Biological Science, ${ }^{c}$ Department of Biomedical Engineering, Michigan Technological University, Houghton, MI 49931

${ }^{d}$ Institute of Chemistry, Academia Sinica, Taipei, Taiwan 11529, Republic of China

\section{Table of Contents}

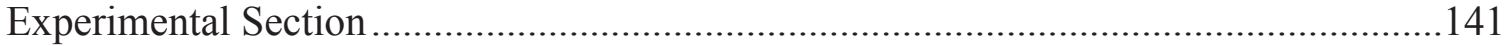

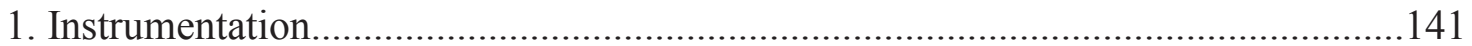

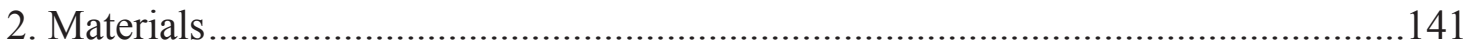

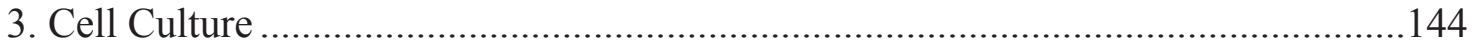

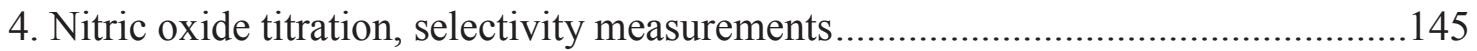

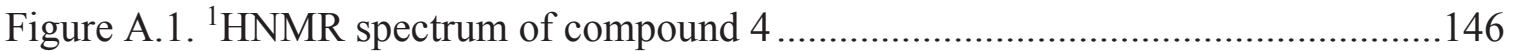

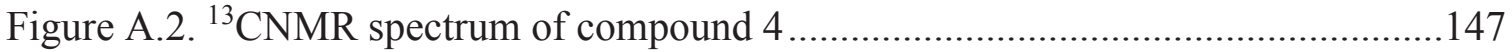

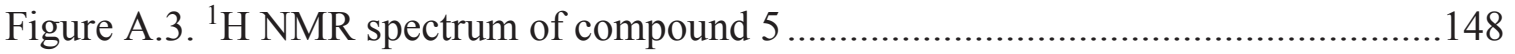

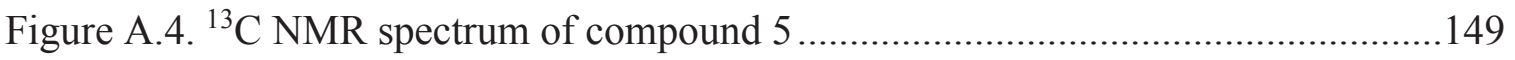

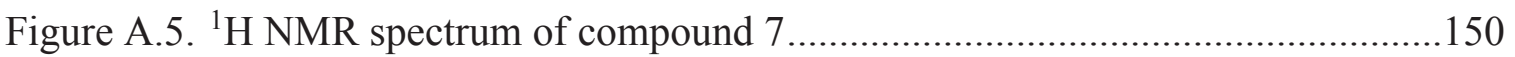

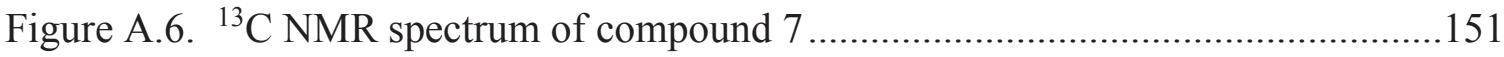

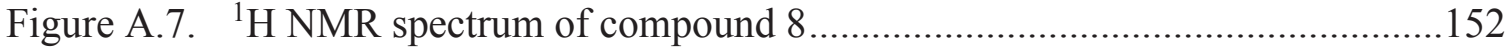

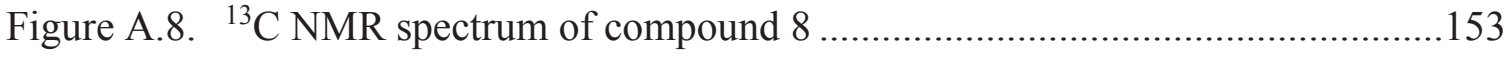

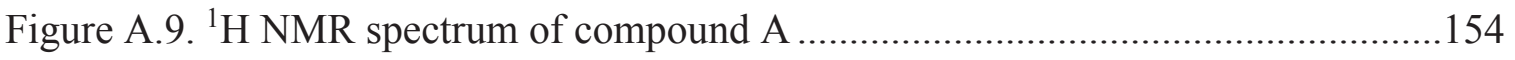

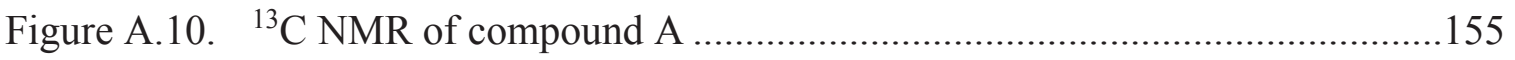

Figure A.11. Normalized absorption and emission spectra of BOIDPY dye 4 in PBS

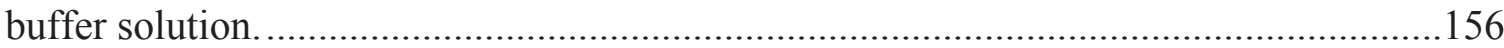

Figure A.12. Normalized absorption and emission spectra of BOIDPY dye 5 in PBS buffer solution. 
Figure A.13. Normalized absorption and emission spectra of BOIDPY dye 8 in

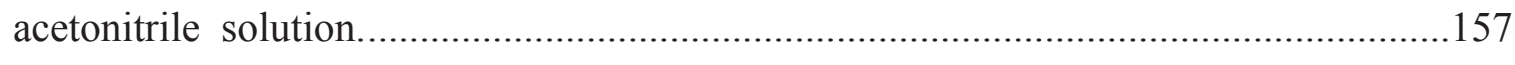

Figure A.14. Normalized absorption and emission spectra of BOIDPY dye A in PBS

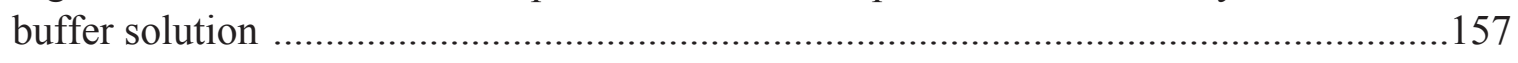

Figure A.15. Cell images of fluorescent probe A .........................................................158

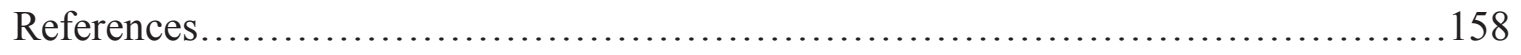




\section{Experimental Section}

1. Instrumentation. ${ }^{1} \mathrm{H}$ NMR and ${ }^{13} \mathrm{C}$ NMR spectra were recorded on a $400 \mathrm{MHz}$ Varian Unity Inova spectrophotometer instrument. ${ }^{1} \mathrm{H}$ and ${ }^{13} \mathrm{C}$ NMR spectra were recorded in $\mathrm{CDCl}_{3}$, and chemical shifts $(\delta)$ are given in ppm relative to solvent peaks (at $7.24 \mathrm{ppm}$ for ${ }^{1} \mathrm{H}$ spectra and at 77.3 for ${ }^{13} \mathrm{C}$ spectra) as internal standard. UV spectra were taken on a Perkin Elmer Lambda 35 UV-Vis spectrometer. Fluorescence spectra were recorded on a Jobin Yvon Fluoromax-4 spectrofluorometer. Fluorescence quantum yields of BODIPY dyes were measured in PBS buffer, acetonitrile and dimethylsulfoxide and calculated by using Rhodamine $6 \mathrm{G}$ excited at $535 \mathrm{~nm}$ in $\mathrm{EtOH}$ as the reference (its quantum efficiency of $95 \%) .{ }^{1-4}$ Both samples and reference dye were prepared and recorded under the same conditions with proper absorbance range from 0.01 to 0.1 at the excitation wavelength for quantum yield calculations. Origin 8.0 was employed to process the integration of the areas for all emission spectra, and plot the graphs of integrated area vs. absorbance. The gradient of the line for the reference and the test sample was obtained by applying a linear fitting. The following equation was used to calculate the quantum yield of each sample: ${ }^{5}$

$$
\Phi_{x}=\Phi_{s t}\left(\operatorname{Grad}_{x} / \operatorname{Grad}_{s t}\right)\left(\eta_{\mathrm{x}}^{2} / \eta_{\mathrm{st}}^{2}\right)
$$

Where the subscripts 'st' and ' $\mathrm{x}$ ' are standard and test, respectively, $\Phi$ stands for the fluorescence quantum yield, Grad represents the gradient from the plot of integrated fluorescence intensity versus absorbance, and $\eta$ is the refractive index of the solvent. Fluorescence lifetimes were measured on a GL-3300 Nitrogen Laser LaserStrobe PTI instrument and analyzed using FeliX32 software.

2. Materials. Unless otherwise indicated, all reagents, and solvents were obtained from commercial suppliers, and were used without further purification. Standard Schlenk line or drybox techniques under a dry argon inert atmosphere was used to conduct air- and moisture-sensitive reactions in oven-dried glassware. Compounds $\mathbf{2}$ and $\mathbf{3}$ were prepared and characterized according to our reported procedure. ${ }^{6}$

Compound 4: Compound 3 ( $3 \mathrm{~g}, 3.34 \mathrm{mmol})$, and 2,4-dimethylpyrrole (0.755 g, 7.34 mmol) were dissolved in $1 \mathrm{~L}$ of dry $\mathrm{CH}_{2} \mathrm{Cl}_{2}$ under a nitrogen atmosphere. When two drops 
of TFA (trifluoroacetic acid) were added to the mixture, resulting reaction mixture was stirred overnight at room temperature in the absence of light. DDQ (2,3-dichloro-5,6dicyano-1,4-benzoquinone) (1.1 g, $5.01 \mathrm{mmol})$ was added to the mixture, and the resulting mixture was further stirred for $3 \mathrm{~h}$ at room temperature. When DIEA (diisopropylethylamine) $(20 \mathrm{~mL})$ and $\mathrm{BF}_{3} . \mathrm{OEt}_{2}(20 \mathrm{~mL})$ were added to the reaction mixture, the resulting mixture was stirred for $30 \mathrm{~min}$. The reaction solution was concentrated to $200 \mathrm{~mL}$ under reduced pressure, and filtered through silica pad. The filtrate was washed with water and brine, dried over anhydrous $\mathrm{MgSO}_{4}$, and concentrated under reduced pressure. The crude product was purified by silica gel column chromatography with hexane/EtOAc/ $\mathrm{CH}_{2} \mathrm{Cl}_{2} / \mathrm{EtOH}(10 / 2 / 6 / 1$, v/v/v/v) to obtain BODIPY dye 4 as yellowred oil (1.49 g, 40\%). ${ }^{1} \mathrm{H}$ NMR (400 MHz, CDCl3): $\delta 6.85$ (d, J = 8.4 Hz, 1H), 6.50-6.45 $(\mathrm{m}, 2 \mathrm{H}), 5.83(\mathrm{~s}, 2 \mathrm{H}), 3.96$ - $3.87(\mathrm{~m}, 4 \mathrm{H}), 3.54-3.41(\mathrm{~m}, 53 \mathrm{H}), 3.27-3.12(\mathrm{~m}, 15 \mathrm{H}), 2.45-$ $2.30(\mathrm{~m}, 7 \mathrm{H}), 2.20-2.15(\mathrm{~m}, 1 \mathrm{H}), 1.38(\mathrm{~s}, 6 \mathrm{H}) .{ }^{13} \mathrm{C}$ NMR (100 MHz, CDCl3): $\delta$ 161.51, $156.66,154.62,142.75,139.45,132.15,129.73,120.72,116.37,106.31,100.35,72.05$, $70.76,70.73,70.62,70.52,70.42,69.48,69.10,66.54,66.21,59.11,40.15,39.64,14.64$, 14.21. IR ( $\left.\mathrm{cm}^{-1}\right): 2868,1608,1543,1509,1467,1410,1363,1351,1303,1275,1261,1192$, 1026, 979, 836, 764, 750, 705. HRMS (FAB) calcd for $\mathrm{C}_{55} \mathrm{H}_{91} \mathrm{~N}_{2} \mathrm{~F}_{2} \mathrm{BO}_{18}[\mathrm{M}+\mathrm{H}]^{+}$, 1116.6328; found, 1116.6332 .

Compound 5: When iodic acid ( $0.173 \mathrm{~g}, 0.984 \mathrm{mmol})$ in $2 \mathrm{~mL}$ of water was added drop wise to ethanol solution $(30 \mathrm{~mL})$ containing compound $4(0.5 \mathrm{~g}, 0.447 \mathrm{mmol})$ and iodine $(0.227 \mathrm{~g}, 0.895 \mathrm{mmol})$ at $0^{\circ} \mathrm{C}$ in 30 minutes, the mixture was stirred at room temperature for $2 \mathrm{~h}$. The reaction solution was concentrated under reduced pressure, diluted with dichloromethane, washed with water and brine solution. The organic layer was collected, dried over $\mathrm{Na}_{2} \mathrm{SO}_{4}$ and concentrated under reduced pressure. The crude compound was purified by silica gel column chromatography using mobile phase of hexane/EtOAc/ $\mathrm{CH}_{2} \mathrm{Cl}_{2} / \mathrm{EtOH}(10 / 2 / 6 / 1, \mathrm{v} / \mathrm{v} / \mathrm{v} / \mathrm{v})$ to yield compound 5 as red oil (0.55 gm, 90\%). ${ }^{1} \mathrm{H}$ NMR (400 MHz, $\left.\mathrm{CDCl}_{3}\right): \delta 6.88(\mathrm{~d}, \mathrm{~J}=8 \mathrm{~Hz}, 1 \mathrm{H}), 6.62-6.52(\mathrm{~m}, 2 \mathrm{H}), 4.05-3.95$ (m, 4H) 3.63-3.50(m, 48H), 3.34-3.16 (m, 20H), 2.59 (s, 6H), 2.44-2.37 (m, 1H), 2.25-2.18 $(\mathrm{m}, 1 \mathrm{H}), 1.48(\mathrm{~s}, 6 \mathrm{H}) .{ }^{13} \mathrm{C} \mathrm{NMR}(100 \mathrm{MHz}, \mathrm{CDCl} 3): \delta 162.03,156.70,156.06,145.06$, 
$139.57,132.14,129.65,116.03,106.53,100.55,85.12,72.14,70.81,70.75,70.58,69.55$, 69.12, 66.70, 66.26, 59.23, 40.20, 39.66, 29.88, 16.74, 16.15. IR ( $\left.\mathrm{cm}^{-1}\right): 2869,1608,1527$, 1457, 1345, 1304, 1178, 1091, 994, 849, 766, 706. HRMS (MALDI) calcd. for $\mathrm{C}_{55} \mathrm{H}_{89} \mathrm{~N}_{2} \mathrm{~F}_{2} \mathrm{BI}_{2} \mathrm{O}_{18} \mathrm{Na}[\mathrm{M}+\mathrm{Na}]^{+}, 1391.4158$; found, 1391.4178 .

Compound 7: After compound $6(0.5 \mathrm{~g}, 1.89 \mathrm{mmol})$, bis(pinacolato)diboron (0.52 g, 2.08 $\mathrm{mmol}), \mathrm{PdCl}_{2}$ (dppf) (41.4 mg, 0.0567 mmol), and KOAc (0.55 g, $\left.5.67 \mathrm{mmol}\right)$ added to a $100-\mathrm{mL}$ round bottom flask containing dry $\operatorname{DMF}(30 \mathrm{~mL})$ under nitrogen atmosphere and the resulting mixture was stirred at $100^{\circ} \mathrm{C}$ overnight. When DMF was removed from the reaction mixture under a reduced pressure, the residue was dissolved in dichloromethane $(50 \mathrm{~mL})$, washed with water and brine solution, respectively. The organic layer was dried over $\mathrm{MgSO}_{4}$ and concentrated under a reduced pressure. The crude product was purified by column chromatography using hexane/ethyl acetate $(3 / 1, v / v)$ to obtain yellowish solid (0.35 g, 70\%). ${ }^{1} \mathrm{H}$ NMR (400 MHz, $\left.\mathrm{CDCl}_{3}\right): \delta 8.57(\mathrm{~d}, \mathrm{~J}=1.2 \mathrm{~Hz}, 1 \mathrm{H}), 7.70(\mathrm{dd}, \mathrm{J}=8,1.2$ $\mathrm{Hz}, 1 \mathrm{H}), 6.75$ (d, J = 8.4 Hz, 1H), 6.19 (s, 2H,NH $) 1.31$ (s, 12H). ${ }^{13} \mathrm{C}$ NMR (100 MHz, CDCl3): $\delta$ 146.62, 141.26, 133.92, 132.38, 118.14, 84.18, 29.90, 25.04 IR $\left(\mathrm{cm}^{-1}\right): 3472$, 3331, 2979, 1625, 1553, 1479, 1372, 1342, 1297, 1250, 1138, 1117, 962, 851, 824, 660. HRMS (ESI) calcd for $\mathrm{C}_{12} \mathrm{H}_{16} \mathrm{BN}_{2} \mathrm{O}_{4}[\mathrm{M}-\mathrm{H}]^{+}, 263.1203$; found, 263.1203 .

Compound 8: When compound 7 (0.289 g, $1.09 \mathrm{mmol}), \mathrm{PdCl}_{2}(\mathrm{dppf})(7.8 \mathrm{mg}, 0.01 \mathrm{mmol})$, and $\mathrm{Na}_{2} \mathrm{CO}_{3}(0.381 \mathrm{~g}, 3.6 \mathrm{mmol})$ were added to a mixed solution of toluene, ethanol and water $(4: 1: 2, \mathrm{v} / \mathrm{v} / \mathrm{v} / \mathrm{v})$ containing compound $5(0.5 \mathrm{~g}, 0.36 \mathrm{mmol})$ under a nitrogen atmosphere, the reaction mixture was stirred at $80^{\circ} \mathrm{C}$ overnight. After the solvent from the mixture was removed under reduced pressure, the resulting residue was dissolved in dichloromethane $(50 \mathrm{~mL})$, and washed with water and brine, respectively. The crude product was purified by column chromatography using a mobile phase of hexane/EtOAc/ $\mathrm{CH}_{2} \mathrm{Cl}_{2} / \mathrm{EtOH}$ (2:3:4:1, v/v/v/v) to get red syrup (0.32 g, 65\%). ${ }^{1} \mathrm{H}$ NMR (400 MHz, CDCl $): \delta 7.89$ (d, J = 2 Hz, 2H), 7.17 (dd, J = 8.4, 2 Hz, 2H), 7.01(d, J = 8.4 $\mathrm{Hz}, 1 \mathrm{H}), 6.86(\mathrm{~d}, \mathrm{~J}=8.4 \mathrm{~Hz}, 2 \mathrm{H}), 6.60(\mathrm{dd}, \mathrm{J}=8.4,2.4 \mathrm{~Hz}, 1 \mathrm{H}), 6.53(\mathrm{~d}, \mathrm{~J}=2 \mathrm{~Hz}, 1 \mathrm{H})$, $6.20\left(\mathrm{~s}, 4 \mathrm{H}, 2 \mathrm{NH}_{2}\right), 4.02(\mathrm{~d}, \mathrm{~J}=5.6 \mathrm{~Hz}, 2 \mathrm{H}), 3.97(\mathrm{~d}, \mathrm{~J}=5.2 \mathrm{~Hz}, 2 \mathrm{H}), 3.62-3.46(\mathrm{~m}, 48 \mathrm{H})$, $3.38-3.25(\mathrm{~m}, 20 \mathrm{H}) 2.50(\mathrm{~s}, 6 \mathrm{H}), 2.43-2.36(\mathrm{~m}, 1 \mathrm{H}), 2.31-2.25(\mathrm{~m}, 1 \mathrm{H}), 1.41(\mathrm{~s}, 6 \mathrm{H}) .{ }^{13} \mathrm{C}$ 
NMR $\left(100 \mathrm{MHz}, \mathrm{CDCl}_{3}\right): \delta 161.76,156.73,153.60,143.93,140.28,13918,137.82$, $132.14,131.26,129.84,127.27,122.67,119.19,116.55,106.49,100.51,72.12,70.83$, $70.73,70.69,70.65,70.64,70.48,69.56,69.28,66.66,66.40,59.21,59.14,40.19,39.68$, 13.53, 12.60. IR $\left(\mathrm{cm}^{-1}\right): 3335,2871,1635,1532,1505,1464,1349,1314,1249,1180$, 1091, 1004, 839, 769, 707. HRMS (MALDI) calcd for $\mathrm{C}_{67} \mathrm{H}_{99} \mathrm{~N}_{6} \mathrm{~F}_{2} \mathrm{BO}_{22} \mathrm{Na}[\mathrm{M}+\mathrm{Na}]^{+}$, 1411.6770; found, 1411.6796.

Fluorescent probe A: When hydrazine monohydrate $(0.4 \mathrm{ml})$ and $10 \% \mathrm{Pd} / \mathrm{C}(7.5 \mathrm{mg}$, $0.0071 \mathrm{mmol})$ was added to ethanol solution $(10 \mathrm{~mL})$ containing the compound $\mathbf{8}(0.1 \mathrm{~g}$, $0.071 \mathrm{mmol}$ ) under a nitrogen atmosphere, the resulting mixture was refluxed under nitrogen atmospheres for one $\mathrm{h}$. The $\mathrm{Pd} / \mathrm{C}$ catalyst was removed by filtration through celite pad. After the solvent was removed from the filtrate under reduced pressure, the crude product was purified on neutral alumina column using a mobile phase of hexane:EtOAC: $\mathrm{CH}_{2} \mathrm{Cl}_{2}: \mathrm{EtOH}(2: 3: 4: 1, \mathrm{v} / \mathrm{v})$ to yield the fluorescent probe $\mathbf{A}(65 \mathrm{mg}$, 68\%). ${ }^{1} \mathrm{H}$ NMR (400 MHz, $\left.\mathrm{CDCl}_{3}\right): \delta 7.00(\mathrm{~d}, \mathrm{~J}=8.4 \mathrm{~Hz}, 1 \mathrm{H}), 6.66(\mathrm{~d}, \mathrm{~J}=8 \mathrm{~Hz}, 2 \mathrm{H}), 6.56$ (dd, J = 8.4, $2.4 \mathrm{~Hz}, 1 \mathrm{H}), 6.51$ (d, J = $2 \mathrm{~Hz}, 1 \mathrm{H}), 6.48$ (d, J = $2 \mathrm{~Hz}, 2 \mathrm{H}), 6.45$ (dd, J = 8, 1.6 $\mathrm{Hz}, 2 \mathrm{H}), 4.02$ (d, J = 6 Hz, 2H), 3.95 (d, J = 5.6 Hz, 2H), 3.61-3.43 (m, 50H), 3.38-3.24 $(\mathrm{m}, 18 \mathrm{H}) 2.48(\mathrm{~s}, 6 \mathrm{H}), 2.42-2.34(\mathrm{~m}, 1 \mathrm{H}), 2.32-2.22(\mathrm{~m}, 1 \mathrm{H}), 1.39(\mathrm{~s}, 6 \mathrm{H}) .{ }^{13} \mathrm{C}$ NMR $(100$ $\left.\mathrm{MHz}, \mathrm{CDCl}_{3}\right): \delta 161.49,156.80,153.57,139.06,138.37,134.81,133.84,133.54,131.96$, $129.98,125.74,122.17,118.58,117.21,116.59,106.25,100.38,72.12,70.83,70.82$, $70.80,70.77,70.73,70.68,70.66,70.50,69.57,69.30,66.60,66.27,59.21,59.17,40.20$, 39.74, 13.58, 12.59. IR ( $\left.\mathrm{cm}^{-1}\right): 3355,2870,1609,1533,1463,1388,1287,1203,1177$, 1096, 1004, 849, 767, 707. HRMS (FAB) calcd for $\mathrm{C}_{67} \mathrm{H}_{103} \mathrm{~N}_{6} \mathrm{~F}_{2} \mathrm{BO}_{18}[\mathrm{M}]^{+}, 1328.7390$; found, 1328.7407.

\section{Cell Culture.}

Murine macrophages (RAW 264.7) and human retinal pigment epithelial cells (ARPE-19) were obtained from ATCC (Manassas, VA). Cells were cultured in $56.7 \mathrm{~cm}^{2}$ dishes (Nunc, Naperville, IL) containing Dulbecco's modified Eagle's medium (DMEM) supplemented with 10\% fetal bovine serum (FBS) (Sigma, St. Louis, MO) and 1\% penicillin/ 
streptomycin (Hyclone, Logan, UT) and cells were maintained in humidified $5 \% \mathrm{CO}_{2}$ incubator at $37^{\circ} \mathrm{C}$. After proliferation, cells were trypsinized with $0.1 \%$ trypsin-EDTA solution (Sigma, St.Louis, MO) for 7-15 min at $37^{\circ} \mathrm{C}$. Cells were seeded in 12 well plates (Nunc) and allowed to grow confluence for one day to perform treatment. Three to eight passage cells were used for experiments.

\section{Nitric oxide titration, selectivity measurements:}

The preparation of nitric oxide (NO) and its stock solutions were carried out according to the reported procedure. ${ }^{7,8} \mathrm{NO}$ gas was generated by slowly adding $6 \mathrm{M} \mathrm{H}_{2} \mathrm{SO}_{4}$ solution into a glass flask containing saturated $\mathrm{NaNO}_{2}$ water. All apparatus were carefully degassed and filled with nitrogen for 30 min for complete removal of oxygen because oxygen can rapidly oxidize $\mathrm{NO}$ to form $\mathrm{NO}_{2}$. The gas formed was passed once through concentrated $\mathrm{NaOH}$ solution and then through distilled water to trap $\mathrm{NO}_{2}$ generated from the reaction of $\mathrm{NO}$ with traces of $\mathrm{O}_{2}$. The NO stock solution was prepared by bubbling $\mathrm{NO}$ gas through $10 \mathrm{~mL}$ of ice-cooled deoxidized water for $1 \mathrm{~h}$ to create a NO-saturated solution. The water solution was deoxidized by bubbling argon through it for $1 \mathrm{~h}$ before introduction of NO. The NO concentration was measured via chemiluminescence with a Sievers 280i Nitric Oxide Analyzer (GE Instruments, Boulder, CO). Stock NO solutions were stored in a glass flask with a rubber septum when they were freshly prepared for each experiment.

The titration of nitric oxide solution to the probe $\mathbf{A}$ in $10 \mathrm{mM}$ phosphate buffer solution (pH 7.0) was conducted via adding small quantity $(0-20 \mu \mathrm{L})$ of ice-cooled NO saturated solution $(2.0 \mathrm{mM})$ into a solution of probe $\mathbf{A}(5 \mu \mathrm{M}, 3 \mathrm{~mL})$ with proper interval. Each sample was analyzed by measuring fluorescence spectrum after 2 min once adding small quantity of NO stock solution.

The relative oxygen and nitrogen species (ROS and RNS) used for selectivity measurement were generated by $\mathrm{H}_{2} \mathrm{O}_{2}: 100 \mu \mathrm{M} \mathrm{H}_{2} \mathrm{O}_{2} ; \mathrm{OCl}^{-}: 100 \mu \mathrm{M} \mathrm{NaOCl} ; \mathrm{NO}_{2}^{-}: 100 \mu \mathrm{M} \mathrm{NaNO}_{2}$; $\mathrm{NO}_{3}{ }^{-}: 100 \mu \mathrm{M} \mathrm{NaNO}_{3}$; $\cdot \mathrm{OH}: 100 \mu \mathrm{M} \mathrm{H}_{2} \mathrm{O}_{2}+100 \mu \mathrm{M}$ ferrous ammonium sulfate; $\mathrm{O}_{2}{ }^{-}: 100$ $\mu \mathrm{M} \mathrm{KO}_{2}$, respectively. 


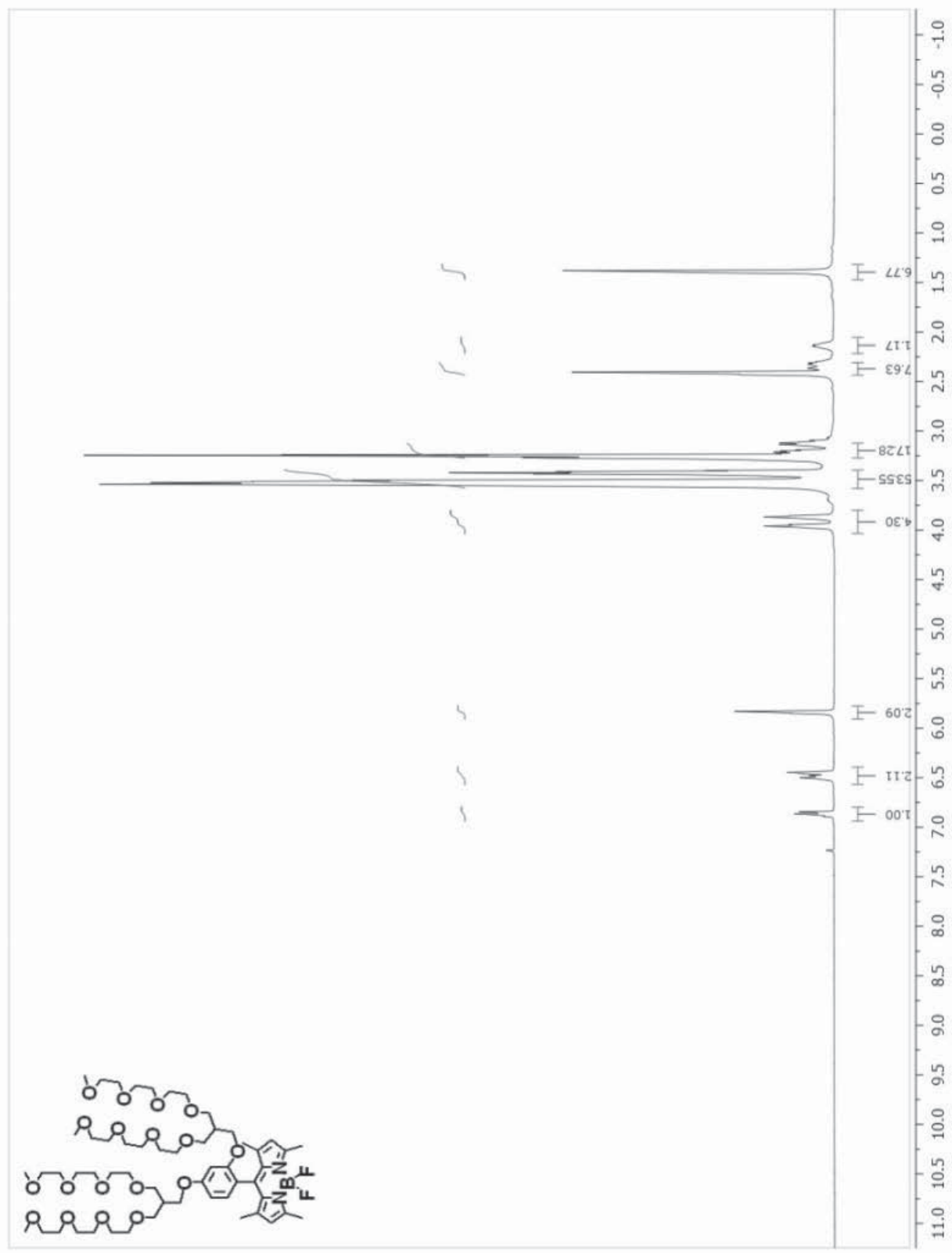

Figure A.1. ${ }^{1} \mathrm{HNMR}$ spectrum of compound 4 


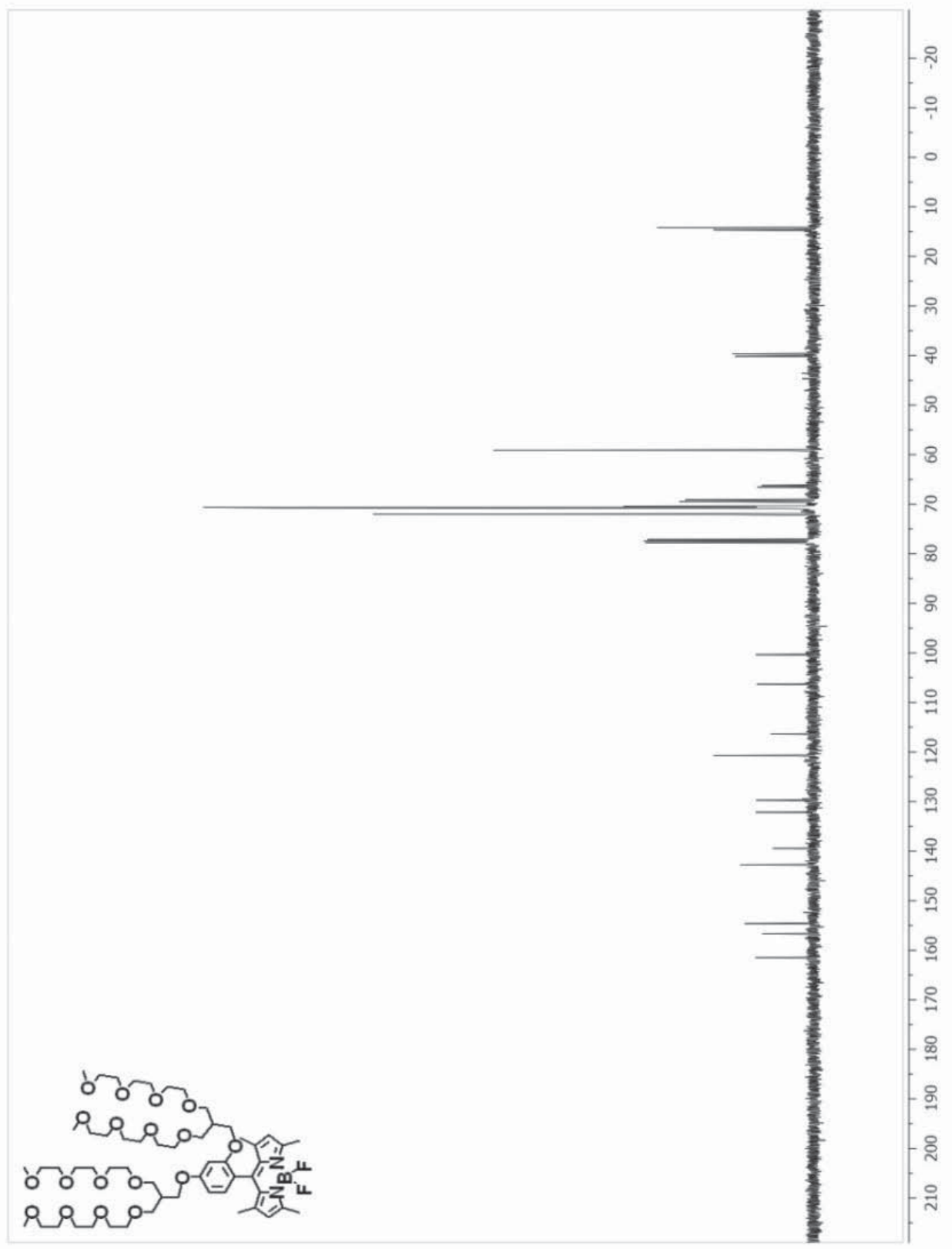

Figure A.2. ${ }^{13} \mathrm{C}$ NMR spectrum of compound 4 


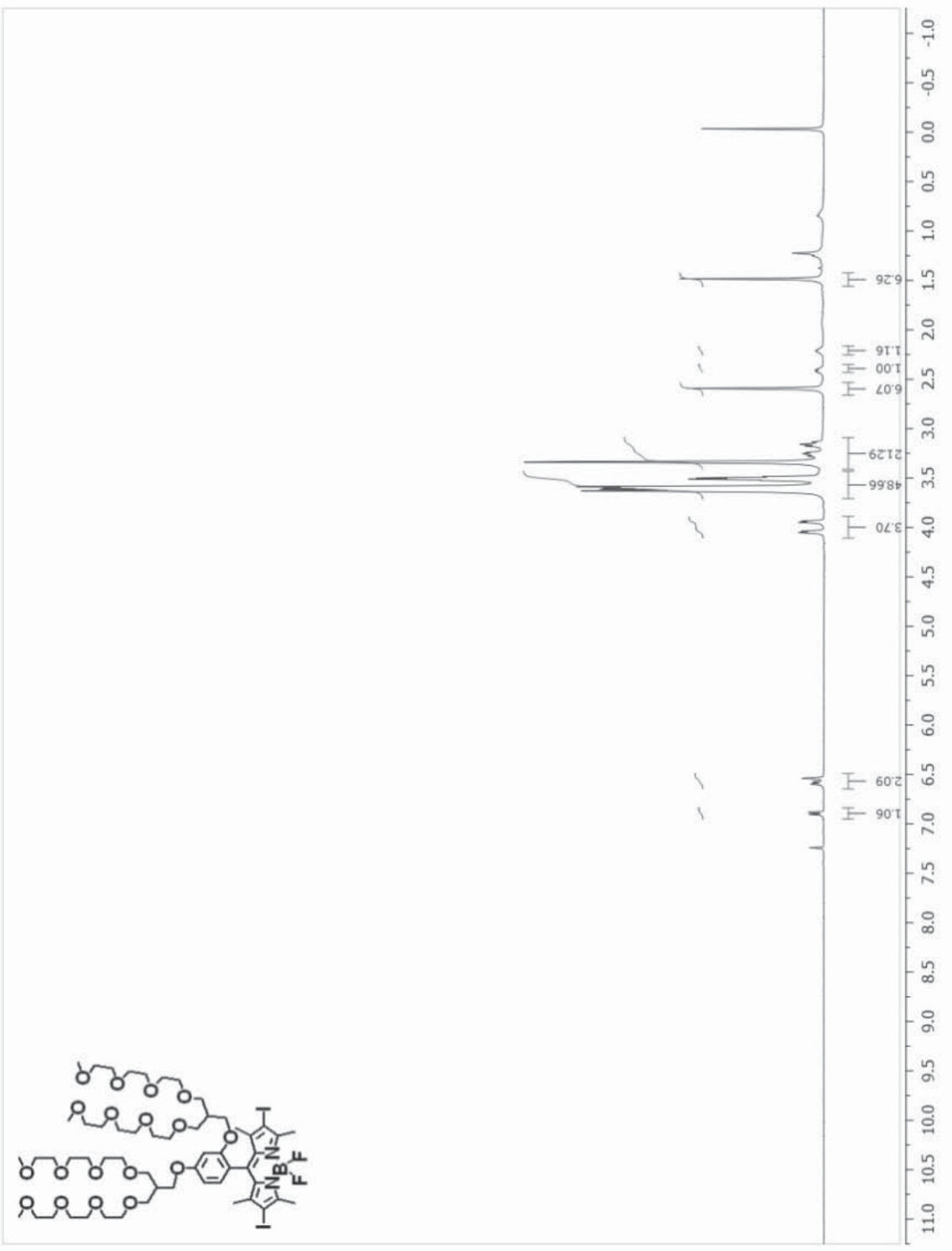

Figure A.3. ${ }^{1} \mathrm{H}$ NMR spectrum of compound $\mathbf{5}$ 


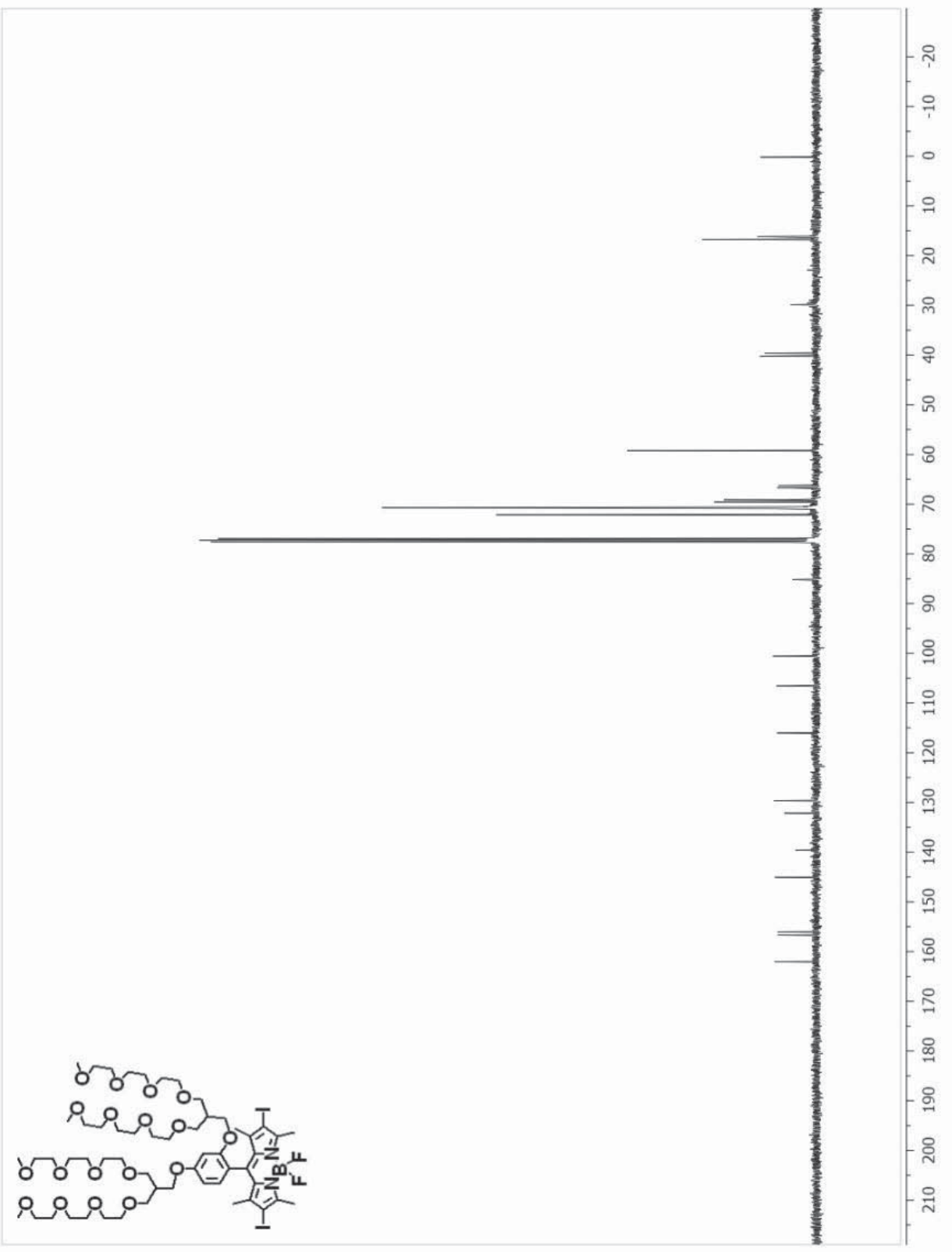

Figure A.4. ${ }^{13} \mathrm{C}$ NMR spectrum of compound 5 


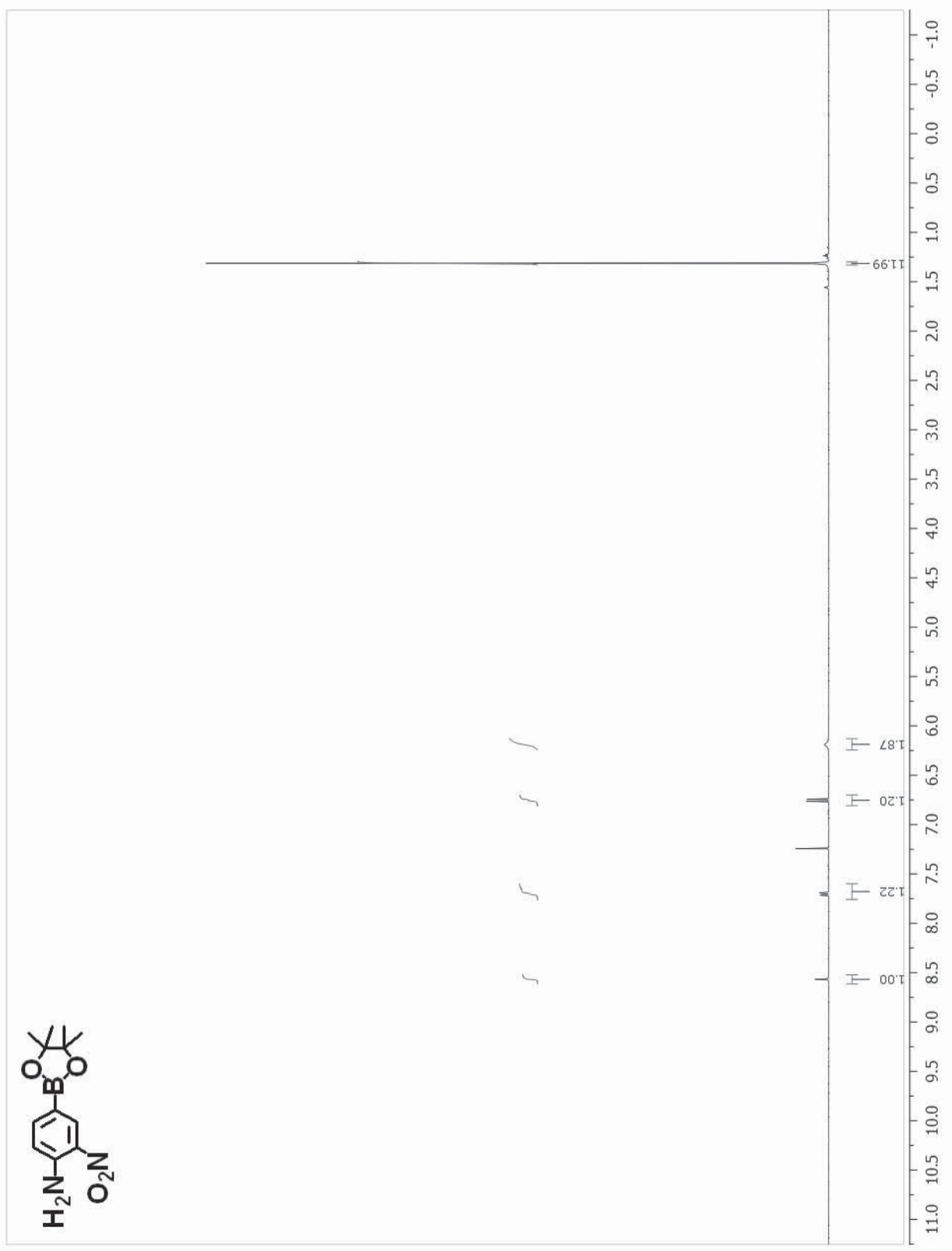

Figure A.5. ${ }^{1} \mathrm{H}$ NMR spectrum of compound 7 


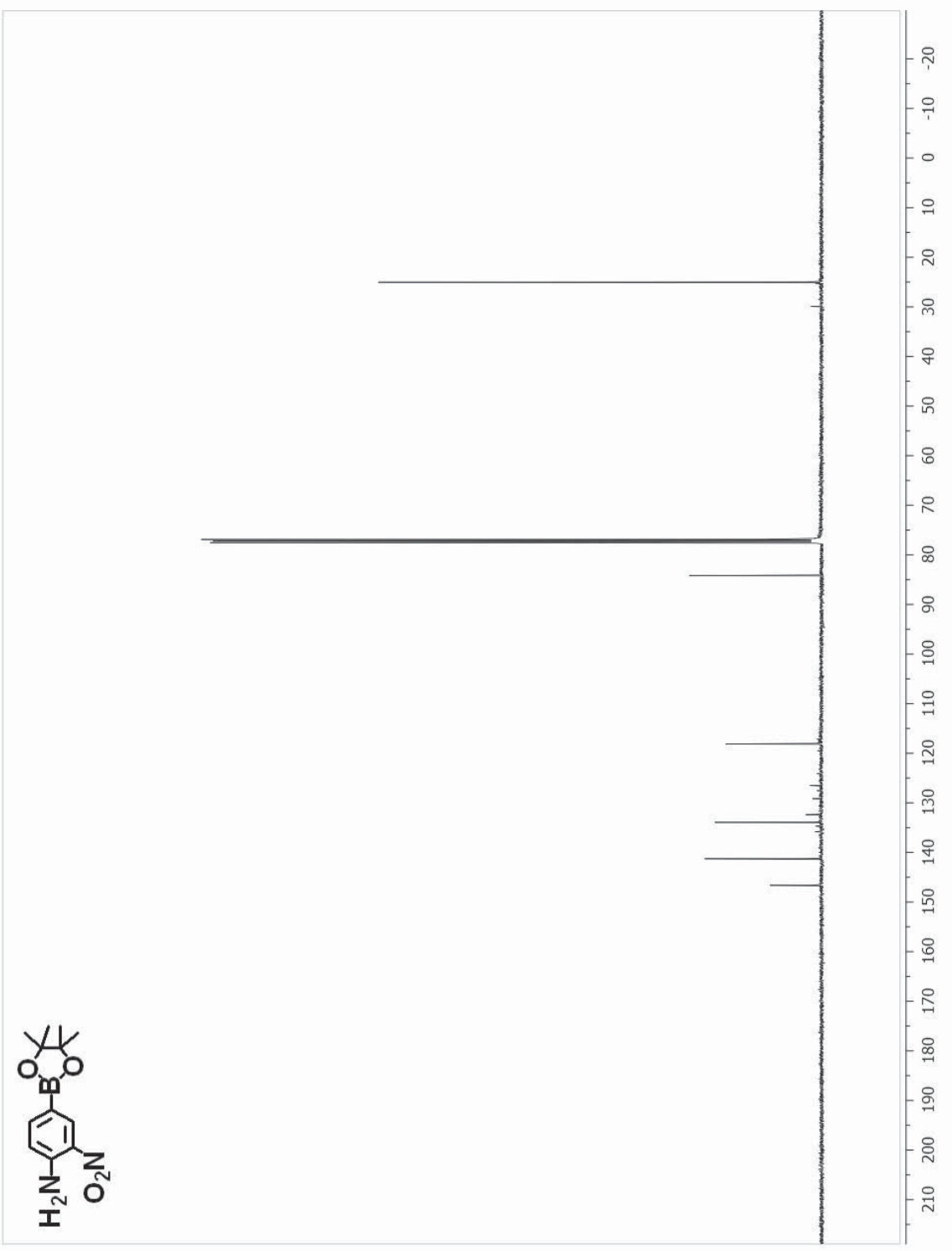

Figure A.6. ${ }^{13} \mathrm{C}$ NMR spectrum of compound 7 


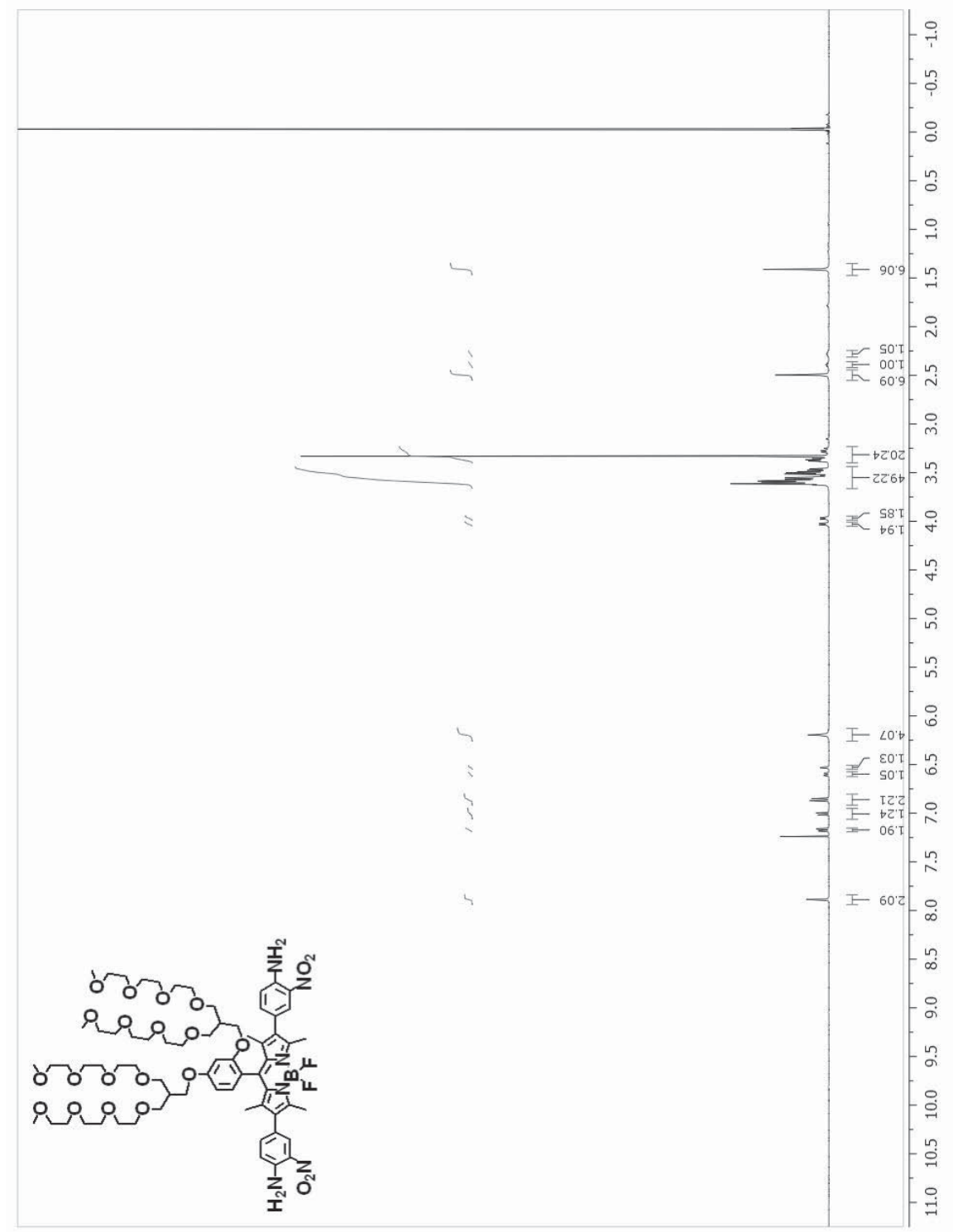

Figure A.7. $\quad{ }^{1} \mathrm{H}$ NMR spectrum of compound $\mathbf{8}$ 


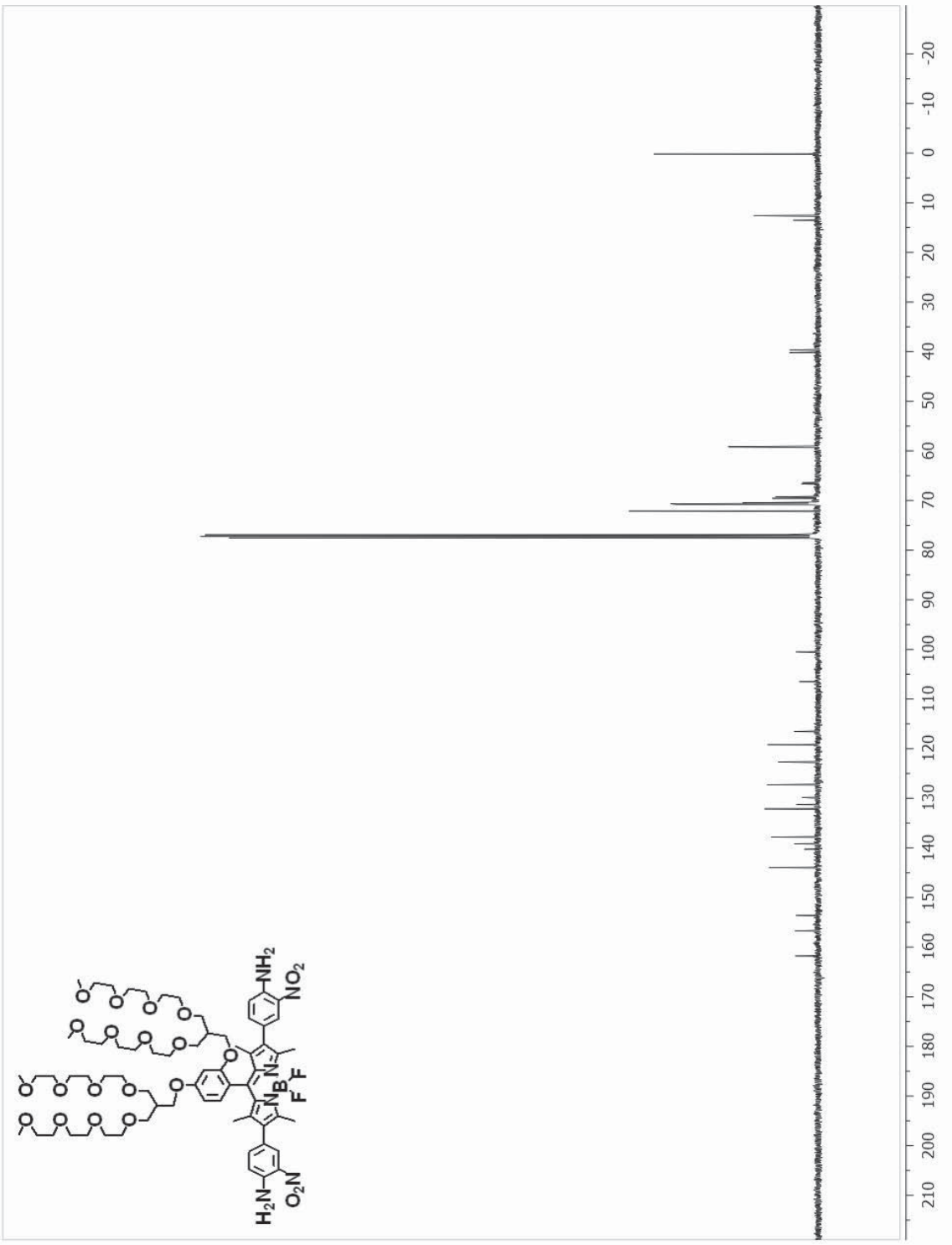

Figure A.8. ${ }^{13} \mathrm{C}$ NMR spectrum of compound $\mathbf{8}$ 


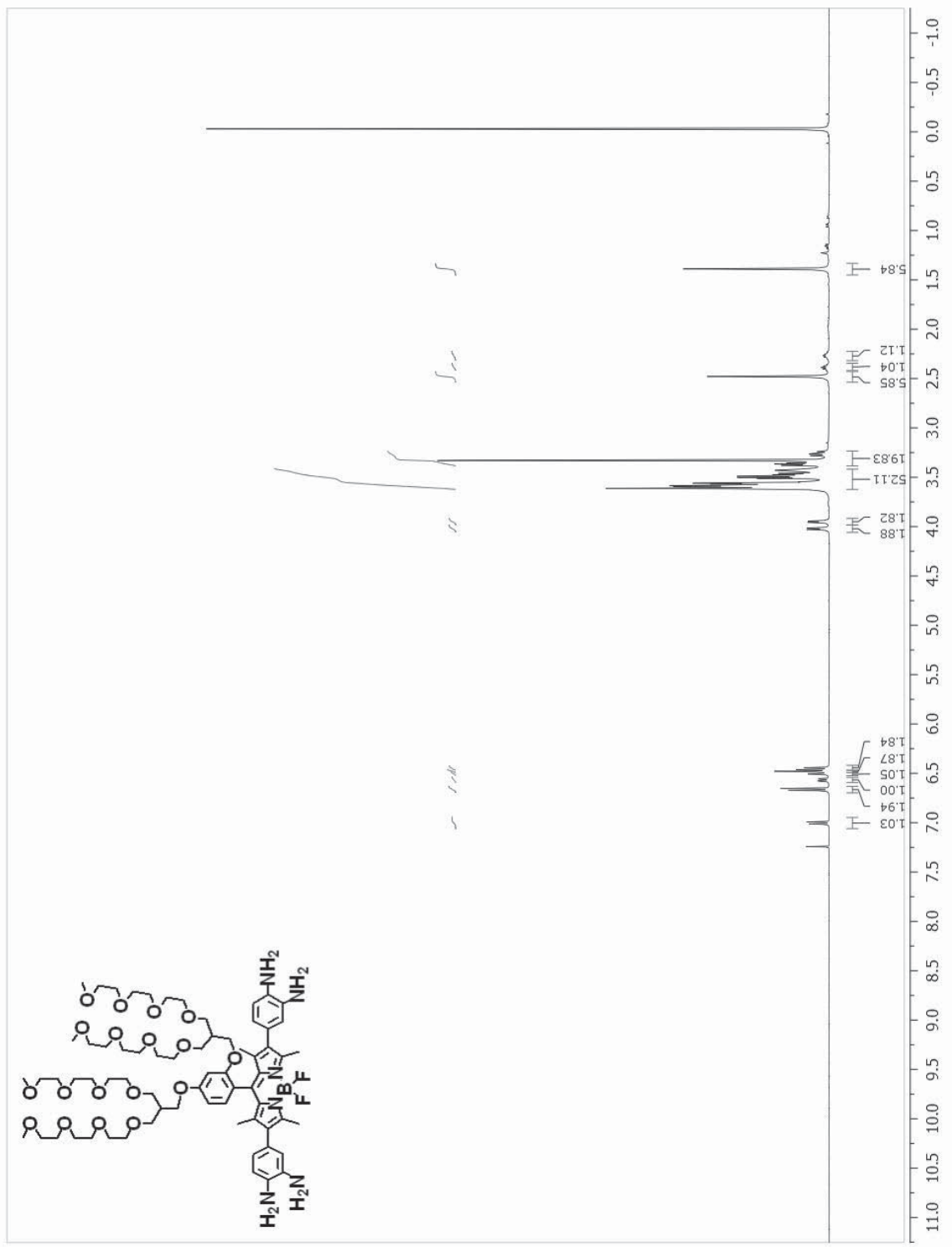

Figure A.9. ${ }^{1} \mathrm{H}$ NMR spectrum of compound A 


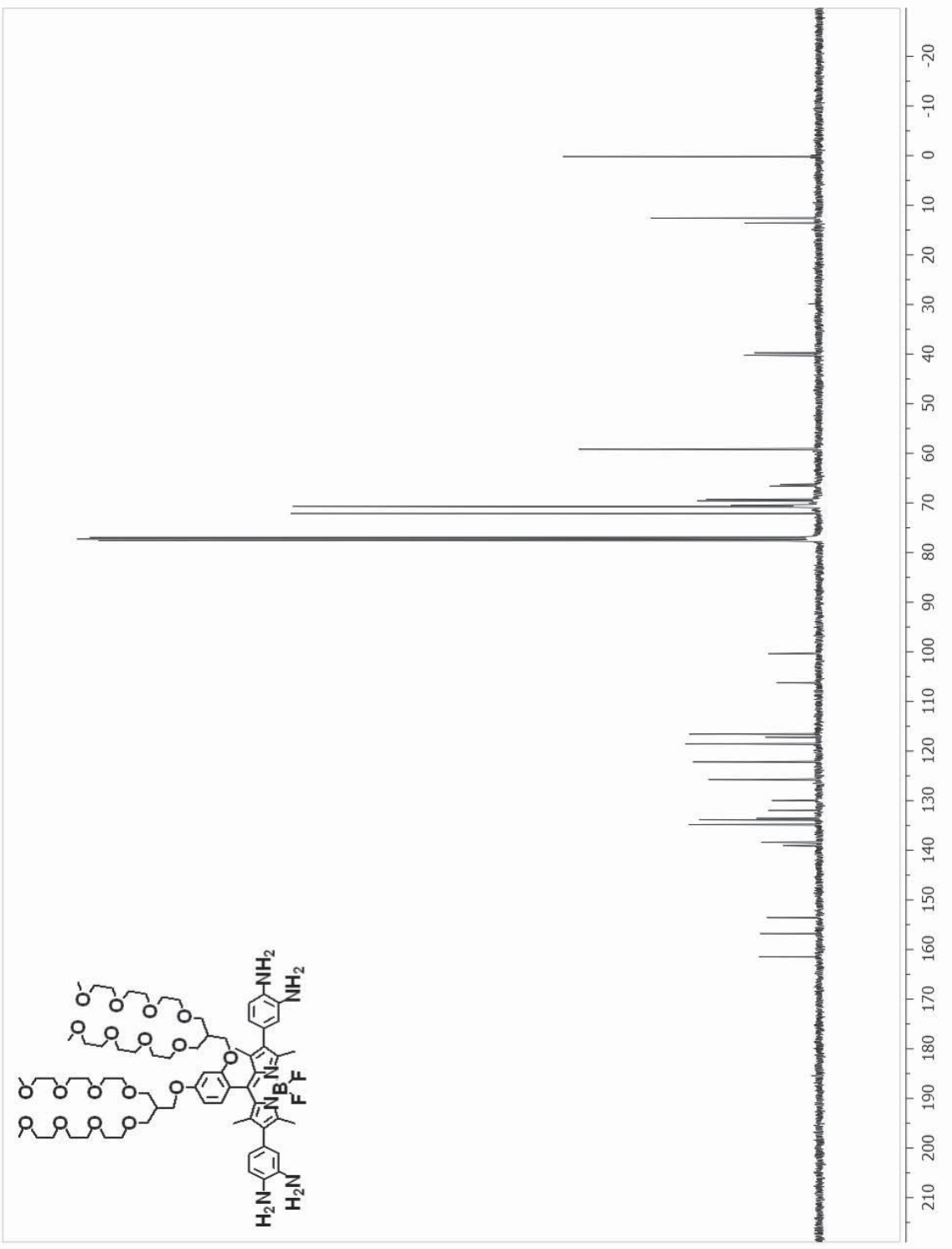

Figure A.10. ${ }^{13} \mathrm{C}$ NMR of compound $\mathbf{A}$ 


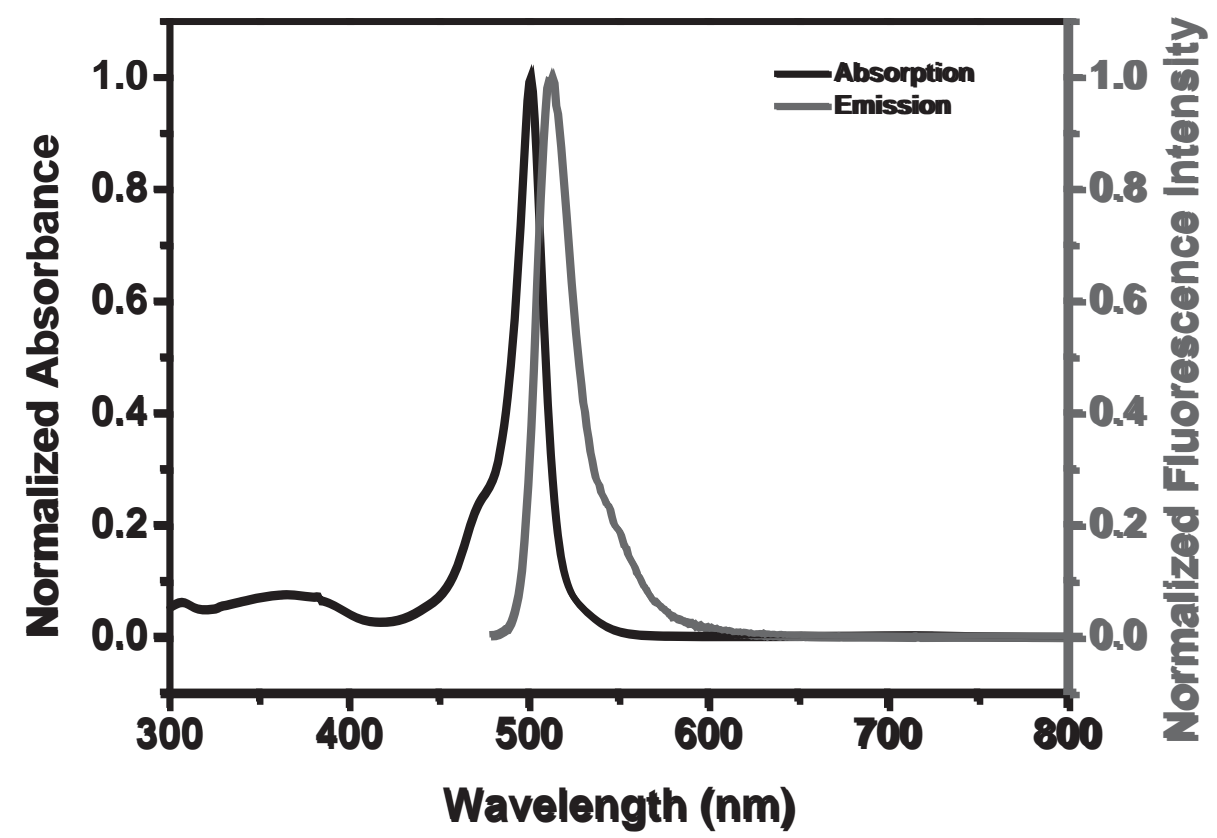

Figure A.11. Normalized absorption and emission spectra of BOIDPY dye 4 in PBS buffer solution (10 mM, pH 7.0).

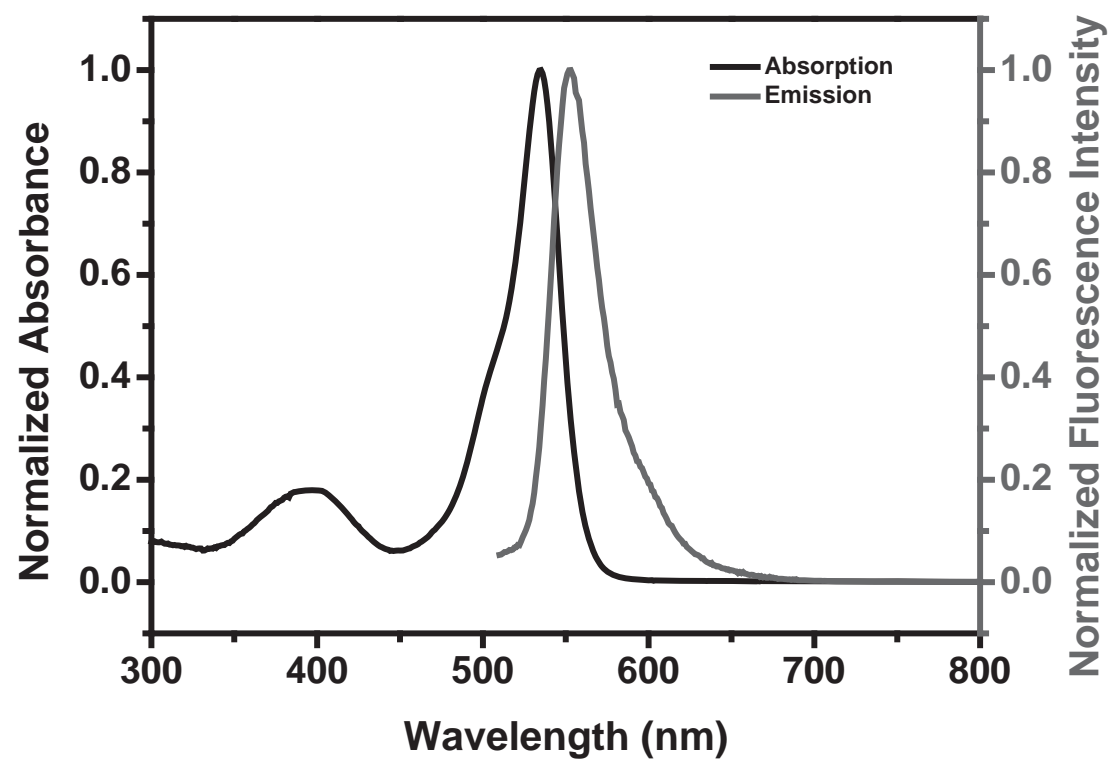

Figure A.12.

Normalized absorption and emission spectra of BOIDPY dye 5 in PBS buffer solution (10 mM, pH 7.0). 


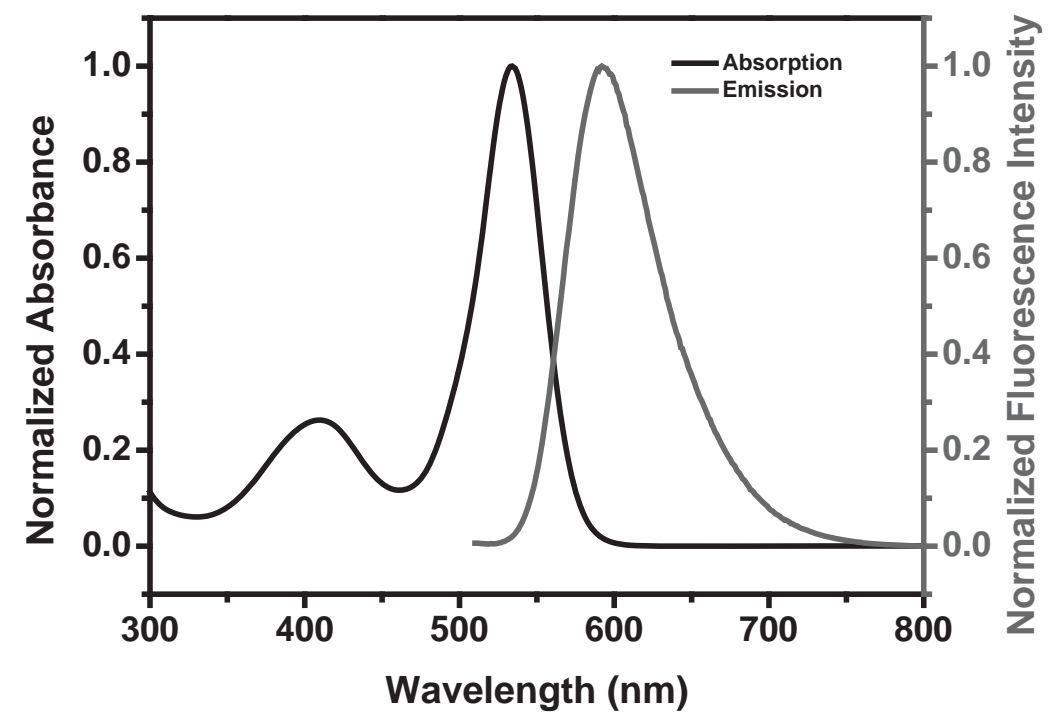

Figure A.13. Normalized absorption and emission spectra of BOIDPY dye $\mathbf{8}$ in acetonitrile solution.

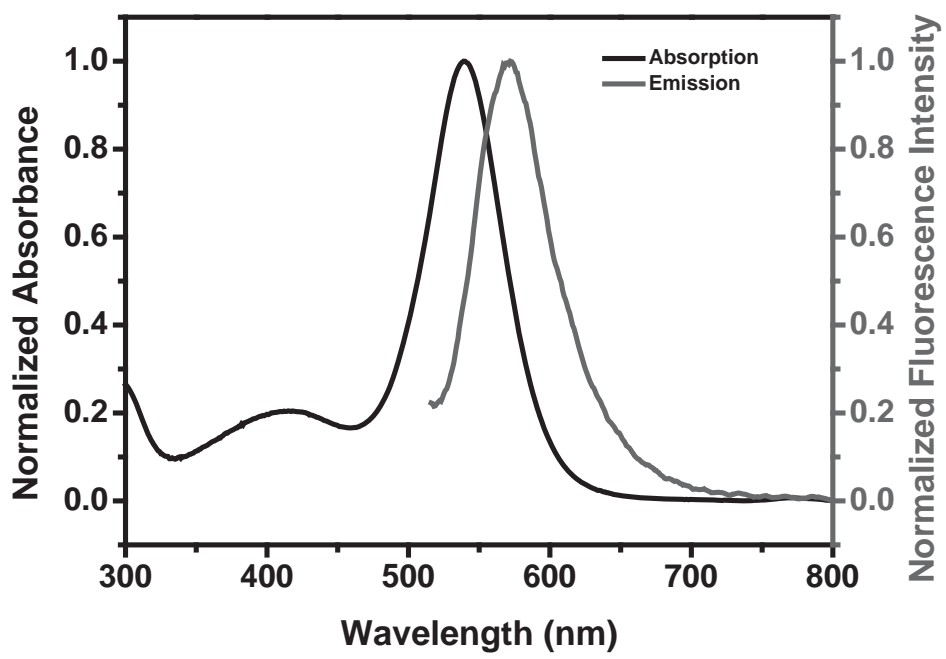

Figure A.14. Normalized absorption and emission spectra of BOIDPY dye A in PBS buffer solution (10 mM, pH 7.0). 

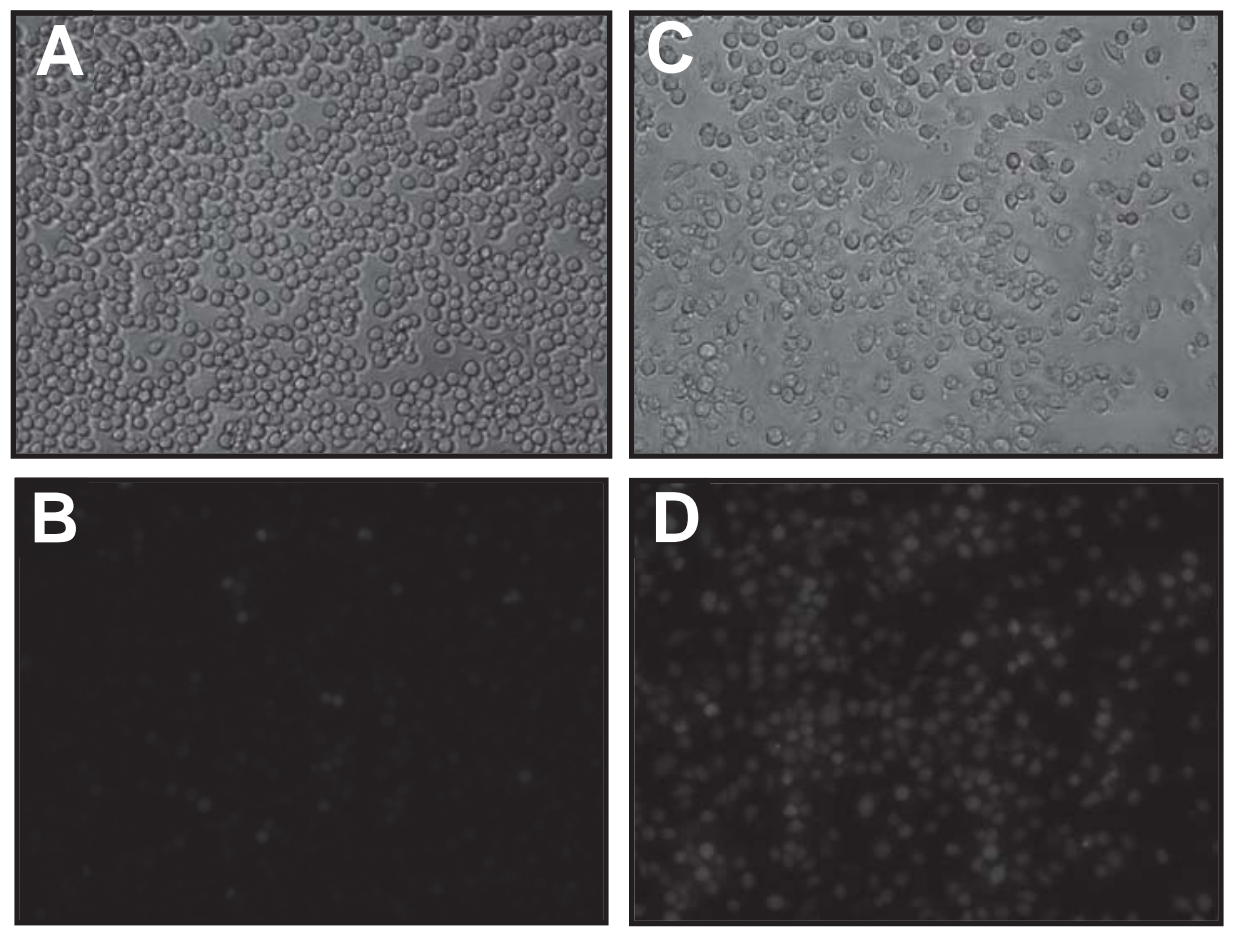

Figure A.15. Bright-field and fluorescence images of murine macrophage RAW264 cells loaded with $10 \mu \mathrm{M}$ 4,5-diaminofluorescein diacetate without and with stimulation of 100 ng/mL liposaccharide. (A) Bright-field,1h, (B) Fluorescence, 1h (C) Bright-field, LPS stimulation, 1h, (D) Fluorescence, LPS stimulation, 1h. Excitation wavelength is at 500 $\mathrm{nm}$.

\section{References}

1. Parker, C. A.; Rees, W. T., Analyst 1960, 85, 587 - 600.

2. Yogo, T.; Urano, Y.; Ishitsuka, Y.; Maniwa, F.; Nagano, T., J. Am. Chem. Soc. 2005, 127, (35), 12162-12163.

3. Maruyama, S.; Kikuchi, K.; Hirano, T.; Urano, Y.; Nagano, T., J. Am. Chem. Soc. 2002, 124, (36), 10650-10651. 
4. Gabe, Y.; Urano, Y.; Kikuchi, K.; Kojima, H.; Nagano, T., J. Am. Chem. Soc. 2004, 126, (10), 3357-3367.

5. Velapoldi, R. A.; Tonnesen, H. H., J. Fluoresc. 2004, 14, (4), 465-472.

6. Zhu, S.; Zhang, J.; Vegesna, G.; Luo, F.-T.; Green, S. A.; Liu, H., Org. Lett. 2011, 13, (3), 438-441.

7. Ouyang, J.; Hong, H.; Shen, C.; Zhao, Y.; Ouyang, C. G.; Dong, L.; Zhu, J. H.; Guo, Z. J.; Zeng, K.; Chen, J. N.; Zhang, C. Y.; Zhang, J. F., Free Radic. Biol. Med. 2008, 45, (10), 1426-1436.

8. Huang, K. J.; Wang, H.; Ma, M.; Zhang, X.; Zhang, H. S., Nitric Oxide-Biol. Ch. 2007, 16, (1), 36-43. 


\section{APPENDIX B \\ SUPPORTING INFORMAYION FOR CHAPTER 3 \\ pH-Activatable Near-Infrared Fluorescent Probes for Detection of Lysosomal pH inside Living Cells}

Giri Vegesna, ${ }^{a}$ Jagadeesh Janjanam, ${ }^{a}$ Jianheng Bi, ${ }^{a}$ Fen-Tair Luo ${ }^{{ }^{*}}$, Connor Olds, Ashutosh Tiwari $^{\text {a* }}$ and Haiying Liu ${ }^{\text {a* }}$

aDepartment of Chemistry, Michigan Technological University, Houghton, MI 49931

b Institute of Chemistry, Academia Sinica, Taipei, Taiwan 11529, Republic of China

\section{Table of Contents}

Figure B.1. ${ }^{1} \mathrm{H}$ NMR spectrum of compound 3 ...................................................... 163

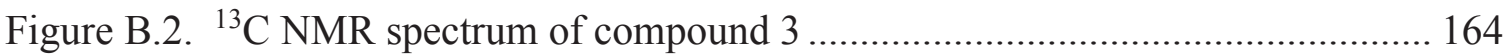

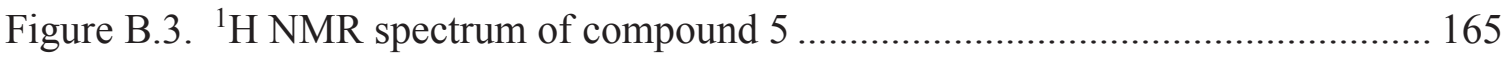

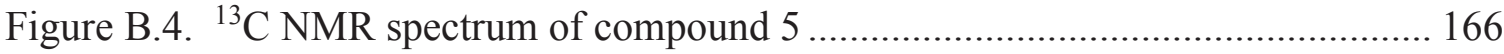

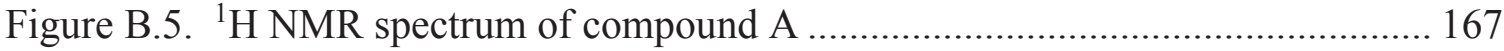

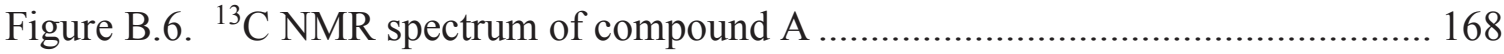

Figure B.7. ${ }^{1}$ H NMR spectrum of compound B.................................................... 169

Figure B.8. ${ }^{13} \mathrm{C}$ NMR spectrum of compound B ................................................ 170

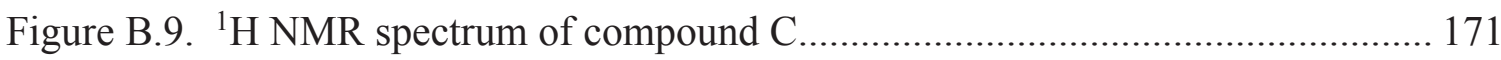

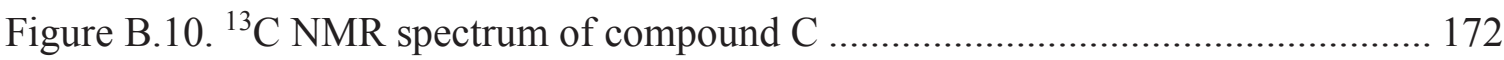

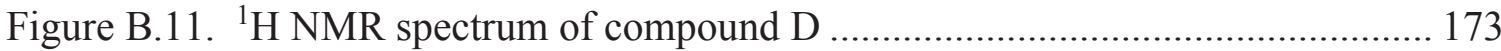

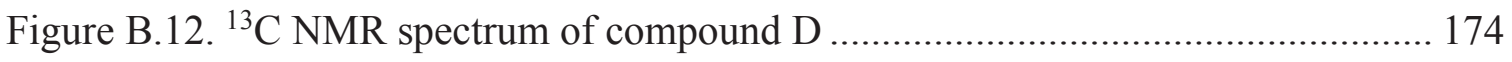

Figure B.13. Normalized absorbance and fluorescence spectra of fluorescent probe A 175

Figure B.14. Normalized absorbance and fluorescence spectra of fluorescent probe B 175

Figure B.15. Normalized absorbance and fluorescence spectra of fluorescent probe C 176

Figure B.16. Normalized absorbance and fluorescence spectra of fluorescent probe D 176

Figure B.17. Absorption spectra of $5 \mu \mathrm{M}$ fluorescent probe $\mathrm{B}$ at different $\mathrm{pH}$ values and

effect of $\mathrm{pH}$ on absorbance of the fluorescent probe $\mathrm{B}$ at $718 \mathrm{~nm}$

Figure B.18. Fluorescence spectra of $5 \mu \mathrm{M}$ fluorescent probe $\mathrm{B}$ at different $\mathrm{pH}$ values

and effect of $\mathrm{pH}$ on fluorescence of the fluorescent probe $\mathrm{B}$ at $743 \mathrm{~nm}$ 177 
Figure B.19. Absorption spectra of $5 \mu \mathrm{M}$ fluorescent probe $\mathrm{C}$ at different $\mathrm{pH}$ values and effect of $\mathrm{pH}$ on absorbance of the fluorescent probe $\mathrm{C}$ at $718 \mathrm{~nm}$............................ 178

Figure B.20. Fluorescence spectra of $5 \mu \mathrm{M}$ fluorescent probe $\mathrm{C}$ at different $\mathrm{pH}$ values and effect of $\mathrm{pH}$ on fluorescence of the fluorescent probe $\mathrm{C}$ at $743 \mathrm{~nm}$

Figure B.21. Fluorescent responses of $5 \mu \mathrm{M}$ fluorescent probe B to different metal ions

Figure B.22. Fluorescent responses of $5 \mu \mathrm{M}$ fluorescent probe $\mathrm{C}$ to different metal ions

Figure B.23. Fluorescent responses of $5 \mu \mathrm{M}$ fluorescent probe $\mathrm{D}$ to different metal ions

Figure B.24. Absorbance spectra of compound 5 at $\mathrm{pH} 4.1$ and 7.0 .......................... 180

Figure B.25. Fluorescence spectra of compound 5 at $\mathrm{pH} 4.1$ and 7.0.

Figure B.26. Fluorescence images of MDA-MB-231 cells incubated with fluorescent

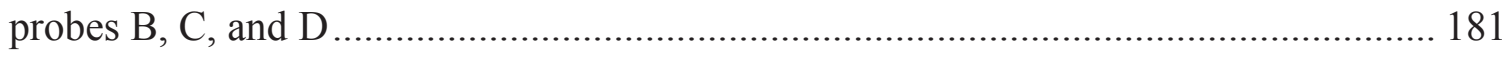

Table B.1: Absorption coefficients and $\mathrm{pK}_{\mathrm{a}}$ values of the probes .............................. 182 


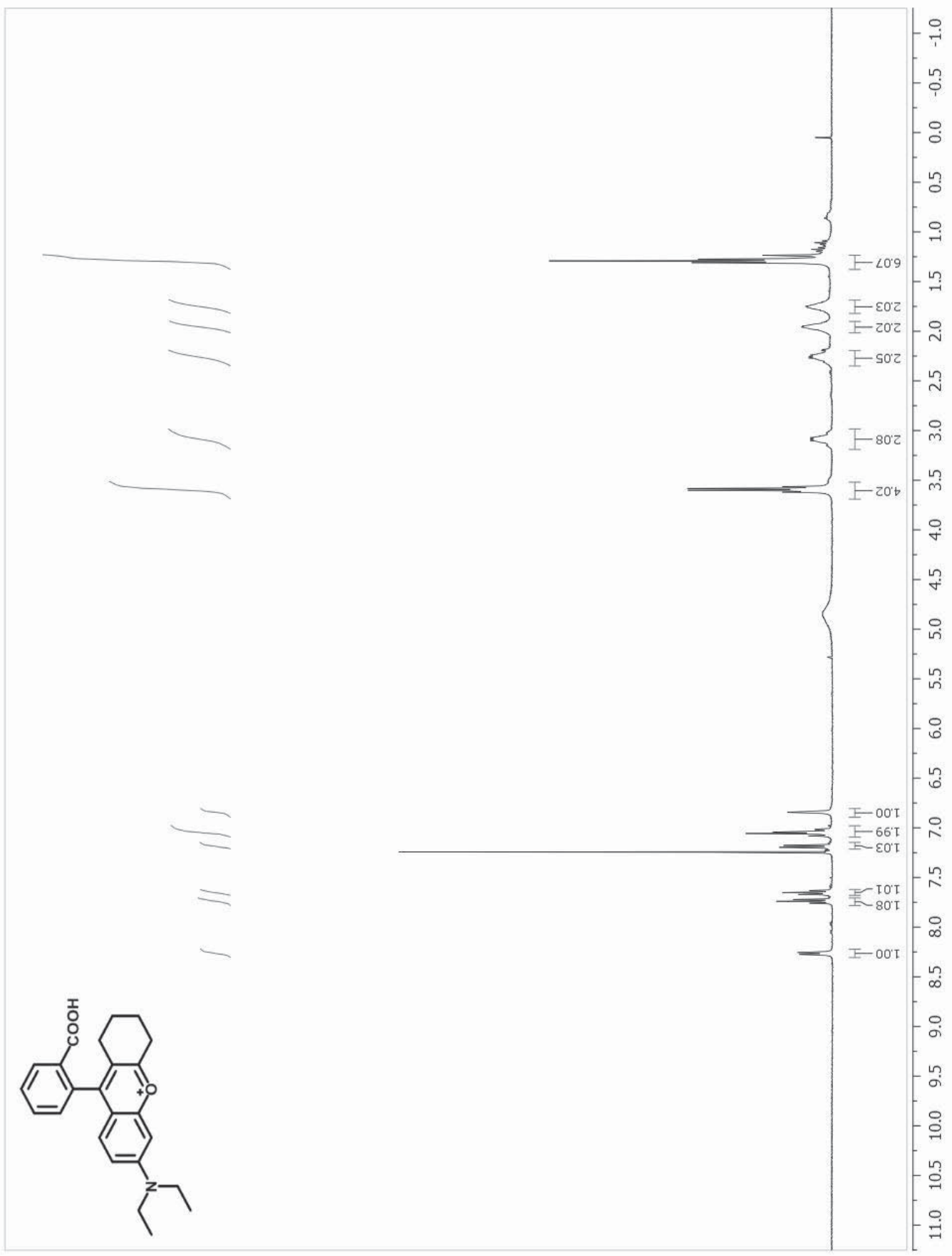

Figure B.1. ${ }^{1} \mathrm{H}$ NMR spectrum of compound $\mathbf{3}$ 


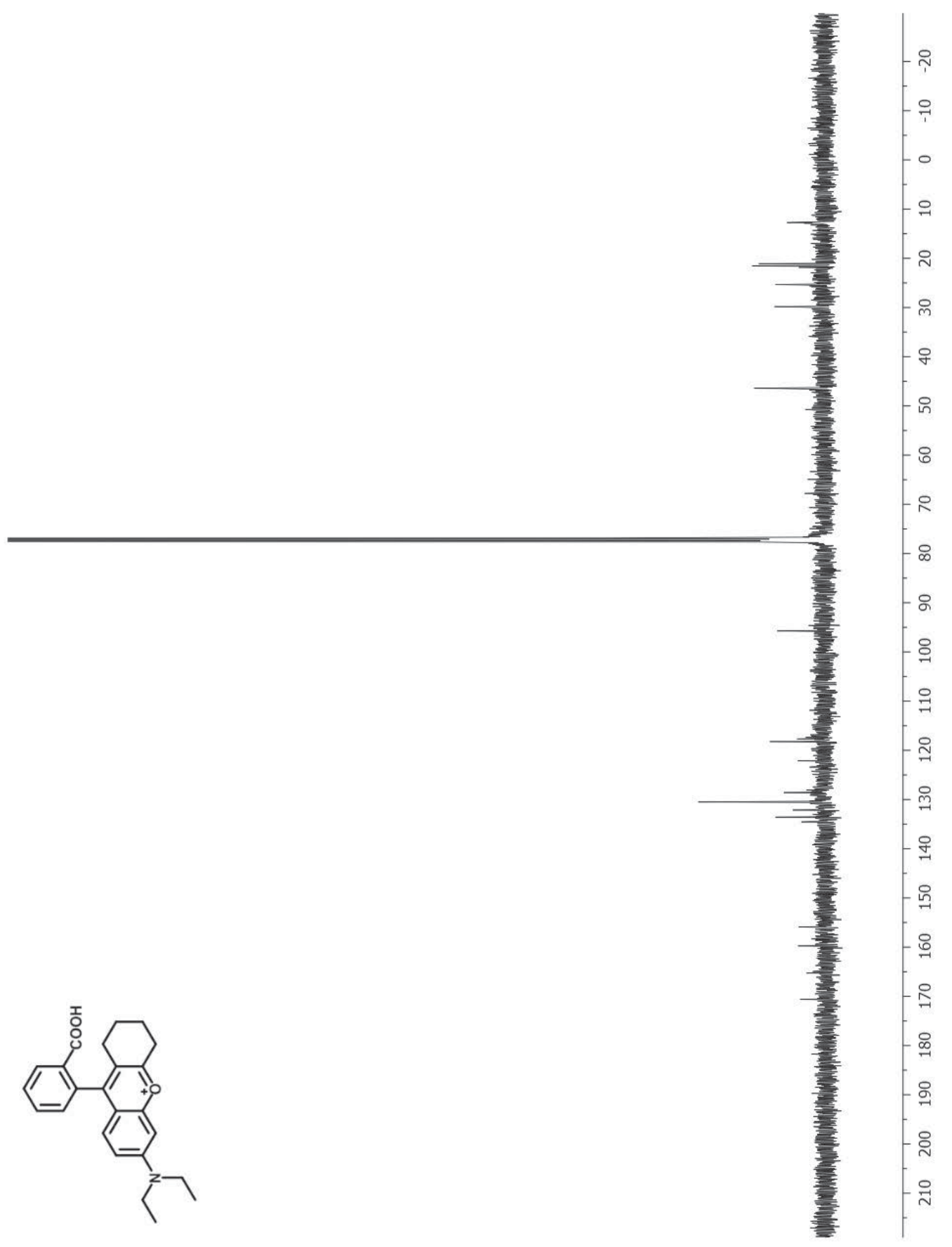

Figure B.2. ${ }^{13} \mathrm{C}$ NMR spectrum of compound $\mathbf{3}$ 


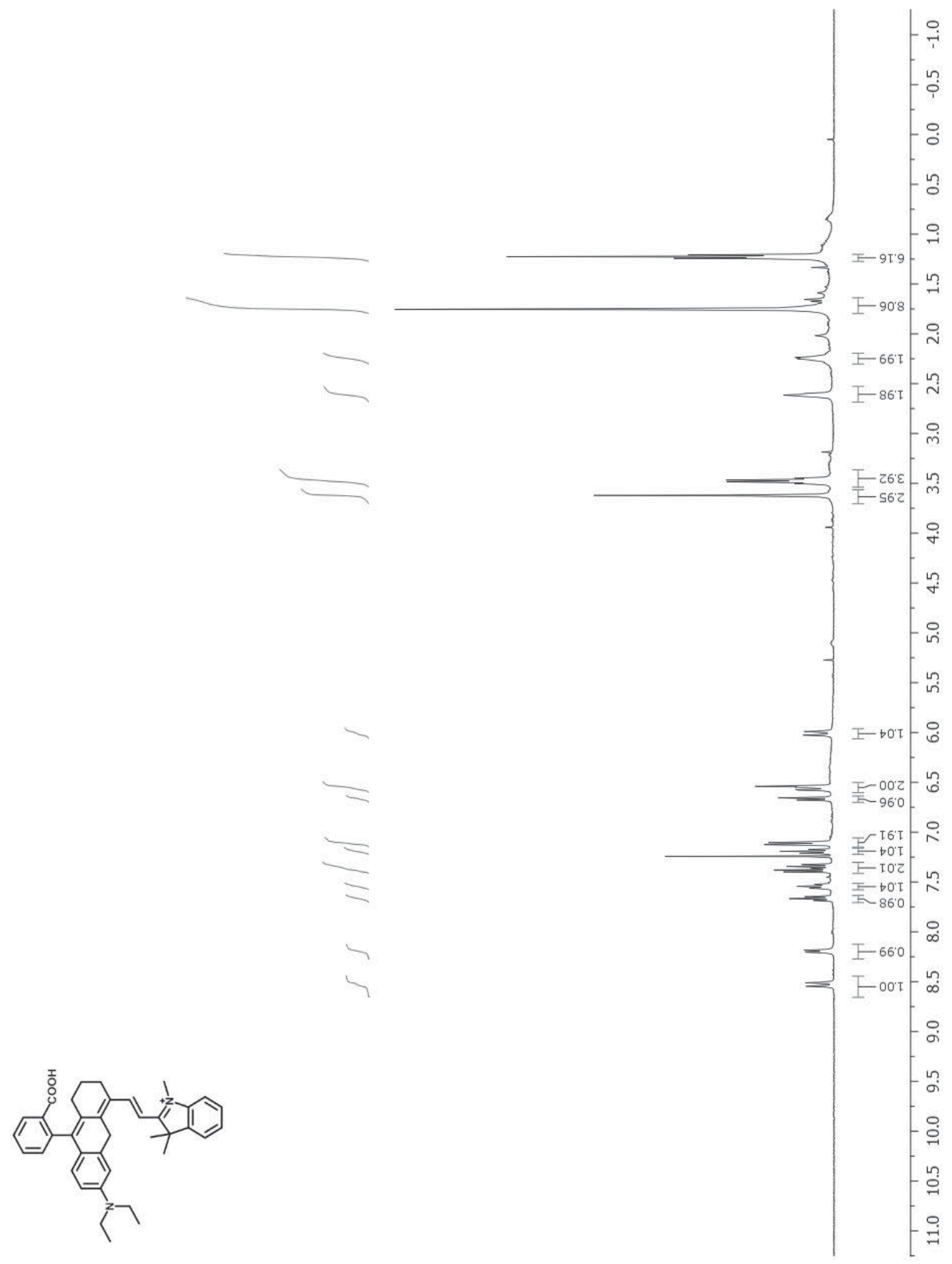

Figure B.3. ${ }^{1} \mathrm{H}$ NMR spectrum of compound 5 


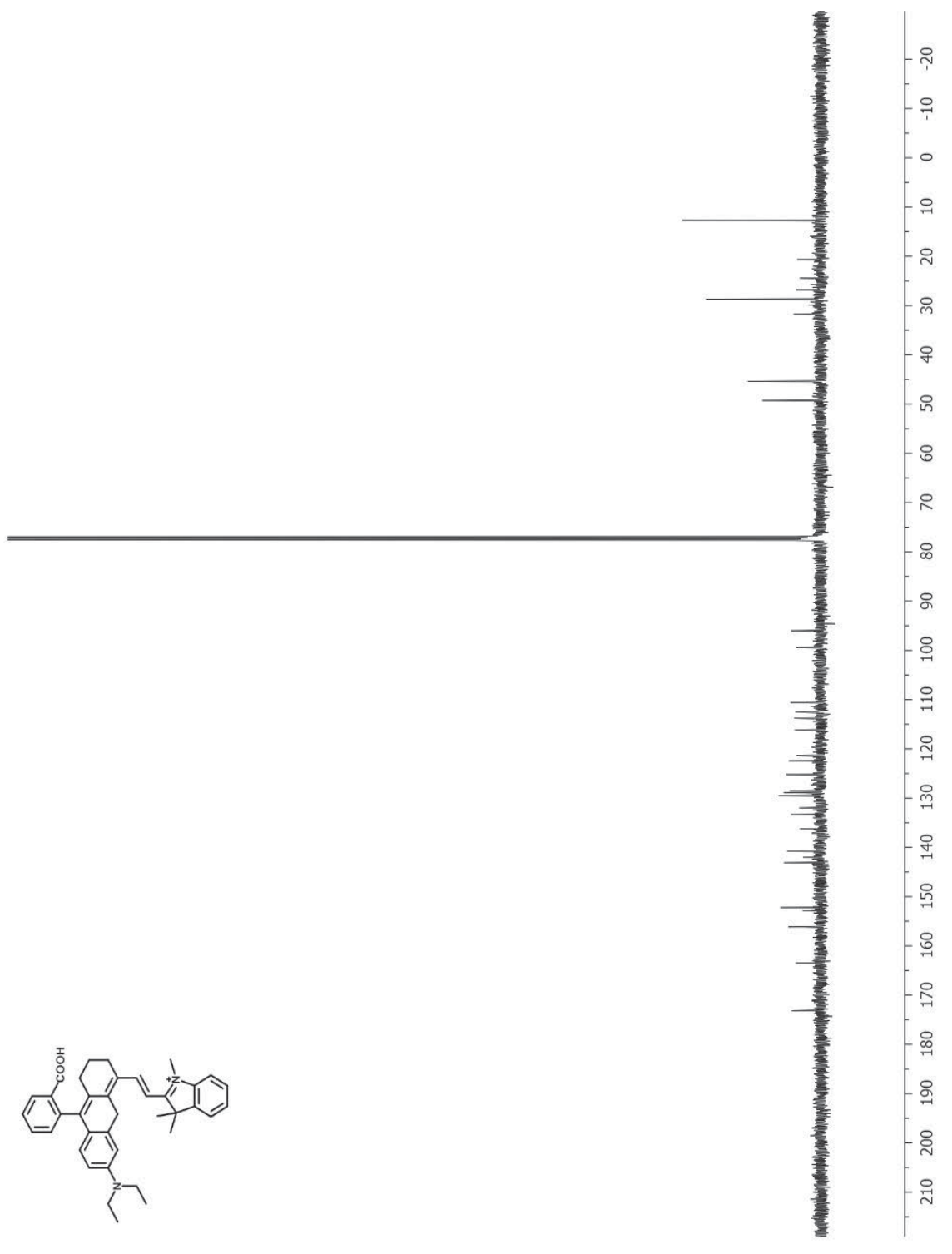

Figure B.4. ${ }^{13} \mathrm{C}$ NMR spectrum of compound $\mathbf{5}$ 


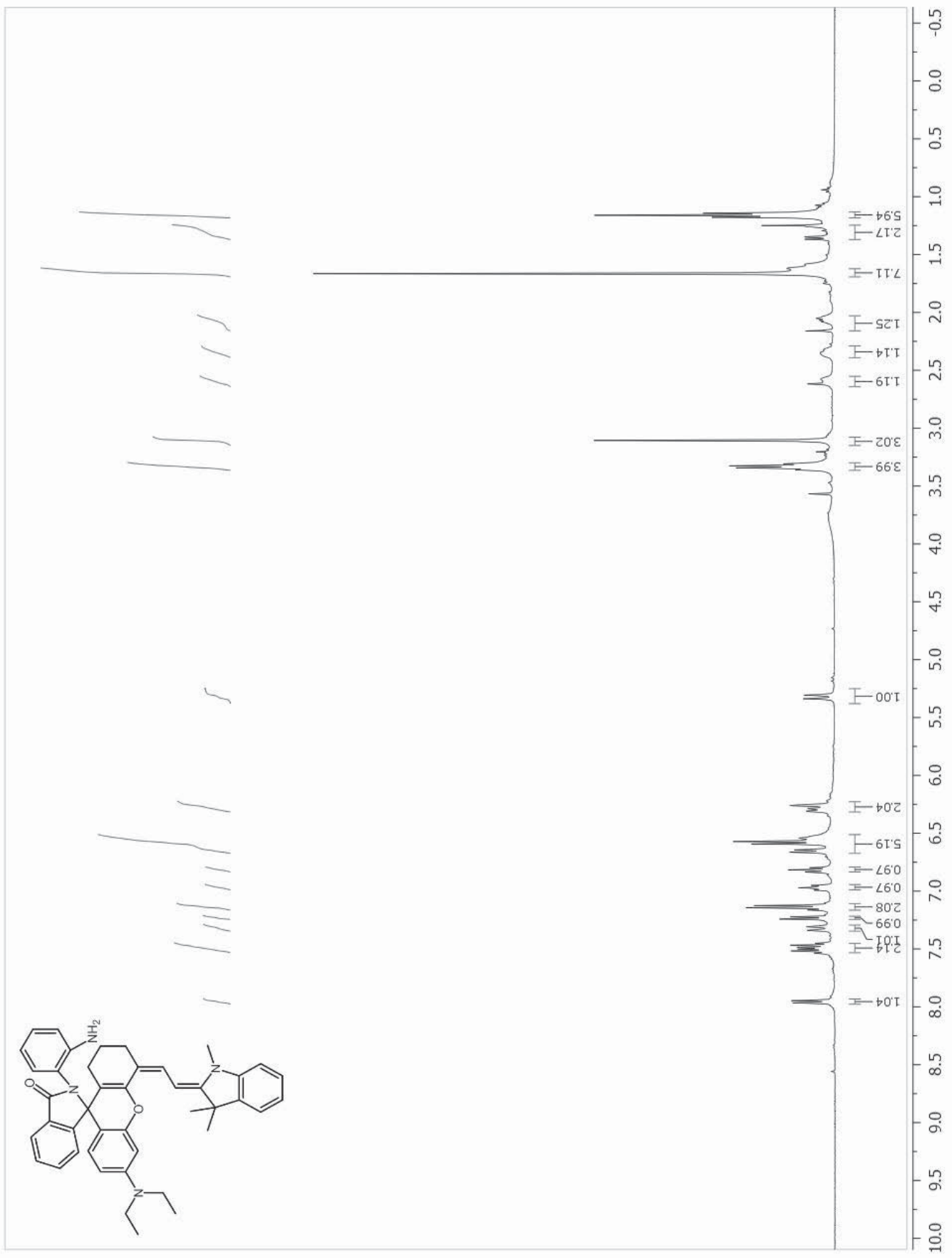

Figure B.5. ${ }^{1} \mathrm{H}$ NMR spectrum of compound A 


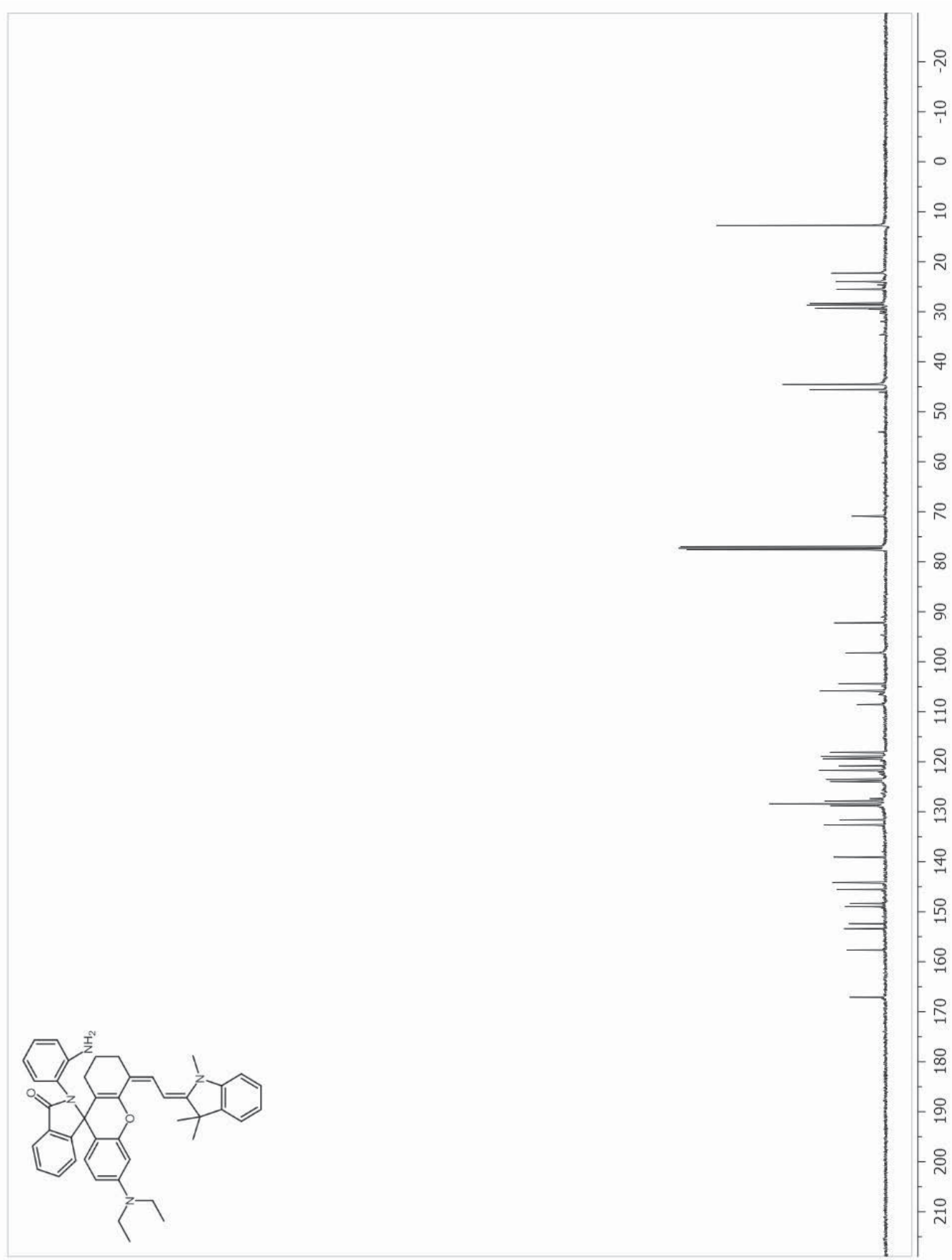

Figure B.6. ${ }^{13} \mathrm{C}$ NMR spectrum of compound A 


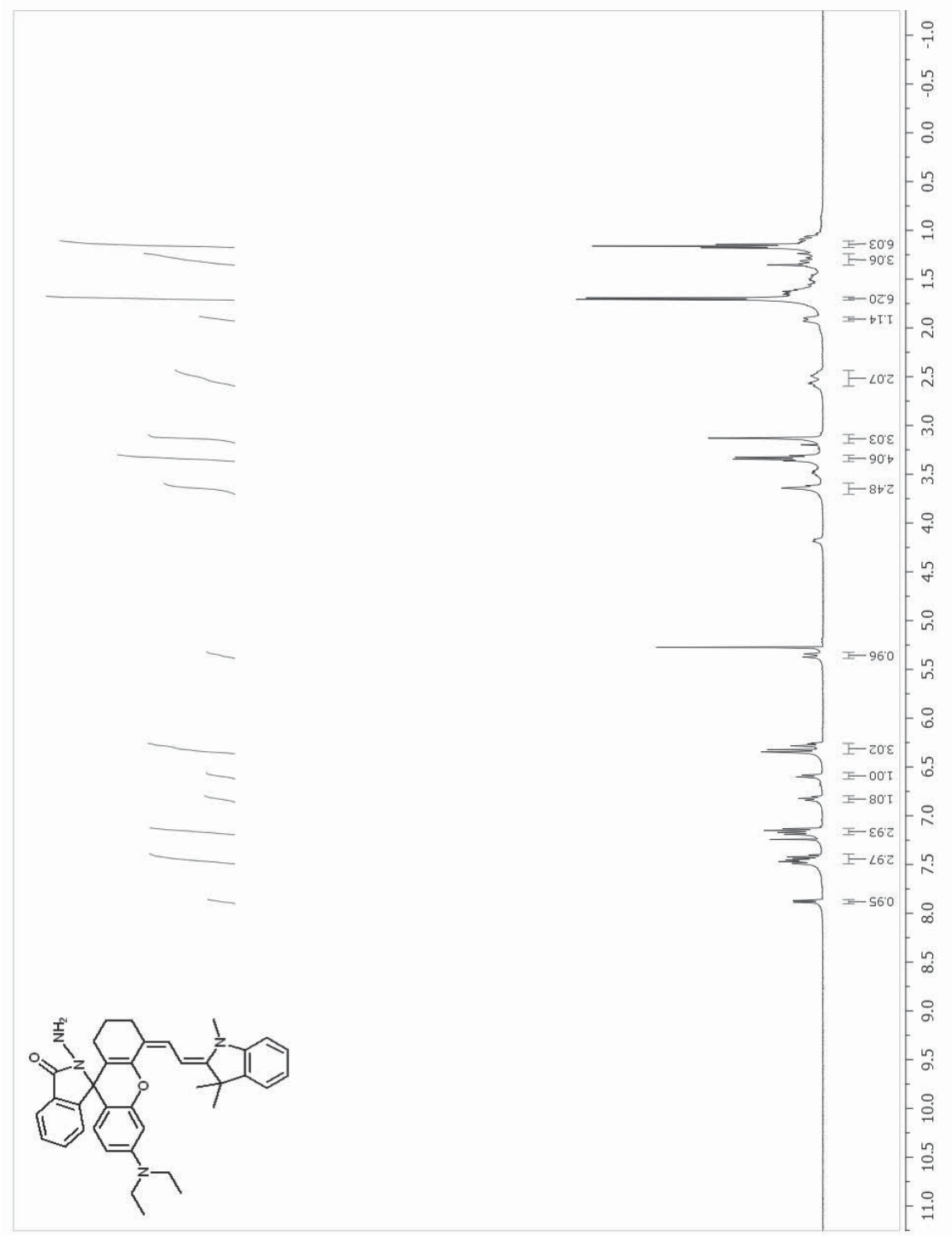

Figure B.7. ${ }^{1} \mathrm{H}$ NMR spectrum of compound B 


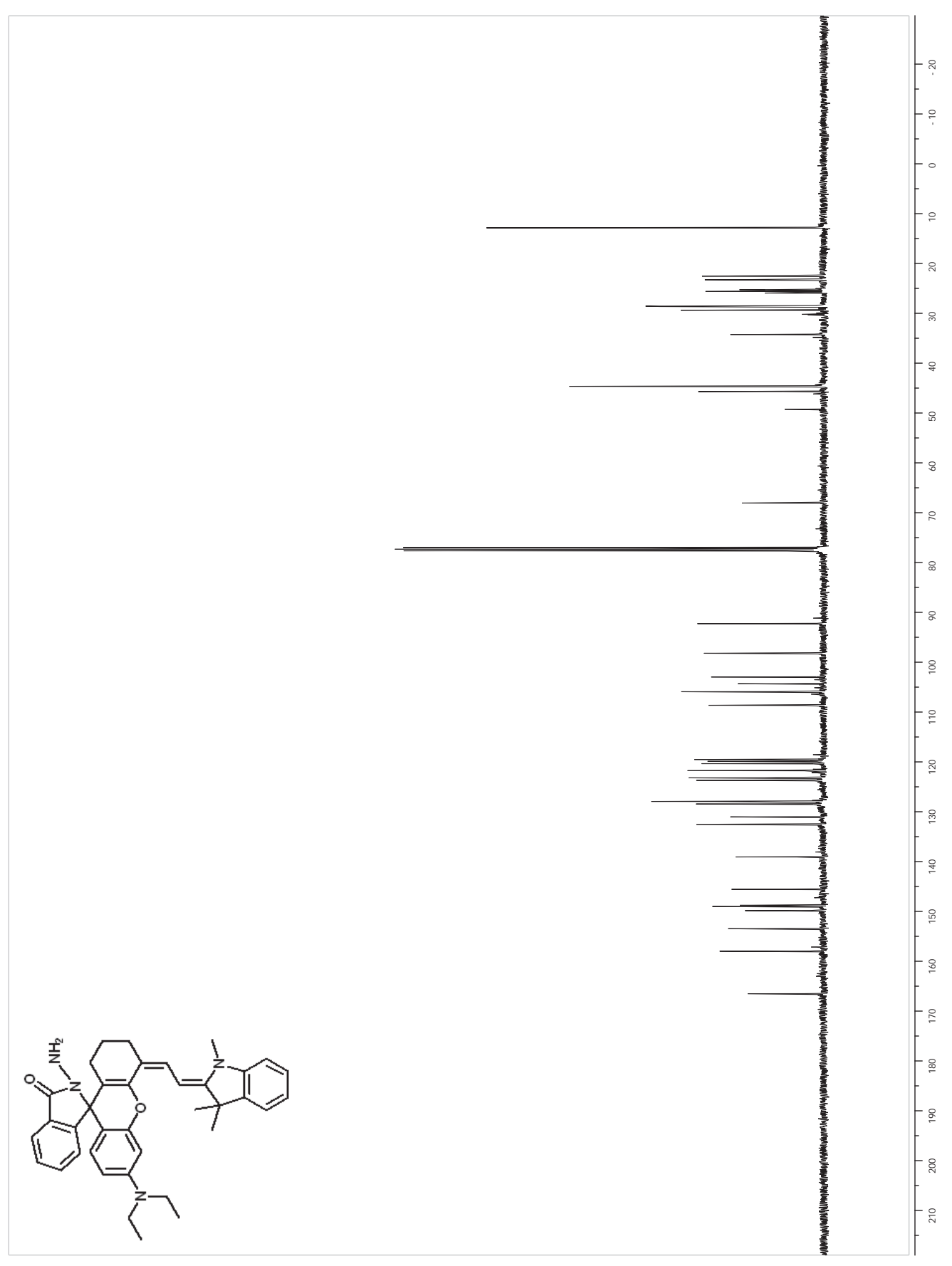

Figure B.8. ${ }^{13} \mathrm{C}$ NMR spectrum of compound $\mathbf{B}$ 


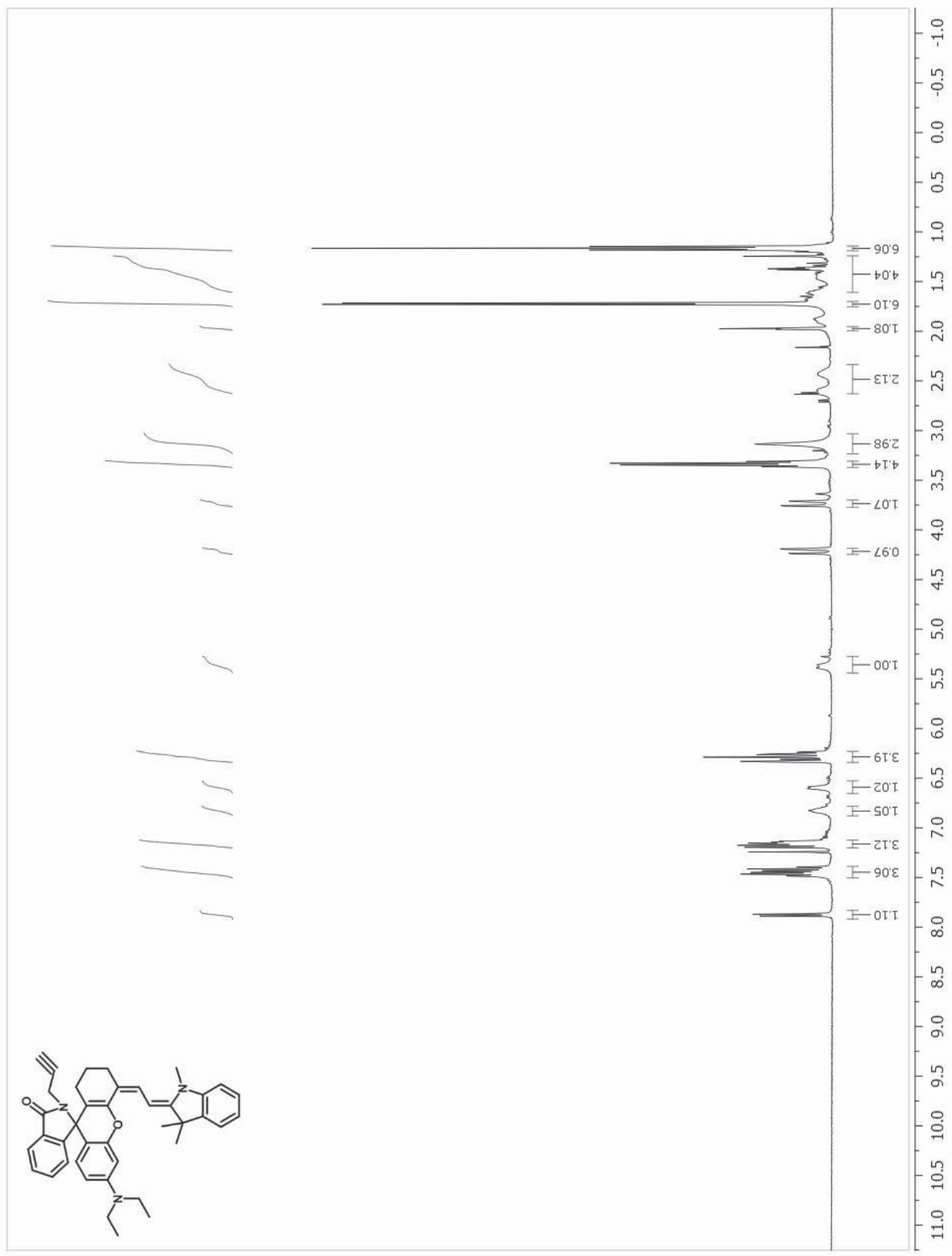

Figure B.9. ${ }^{1} \mathrm{H}$ NMR spectrum of compound $\mathbf{C}$ 


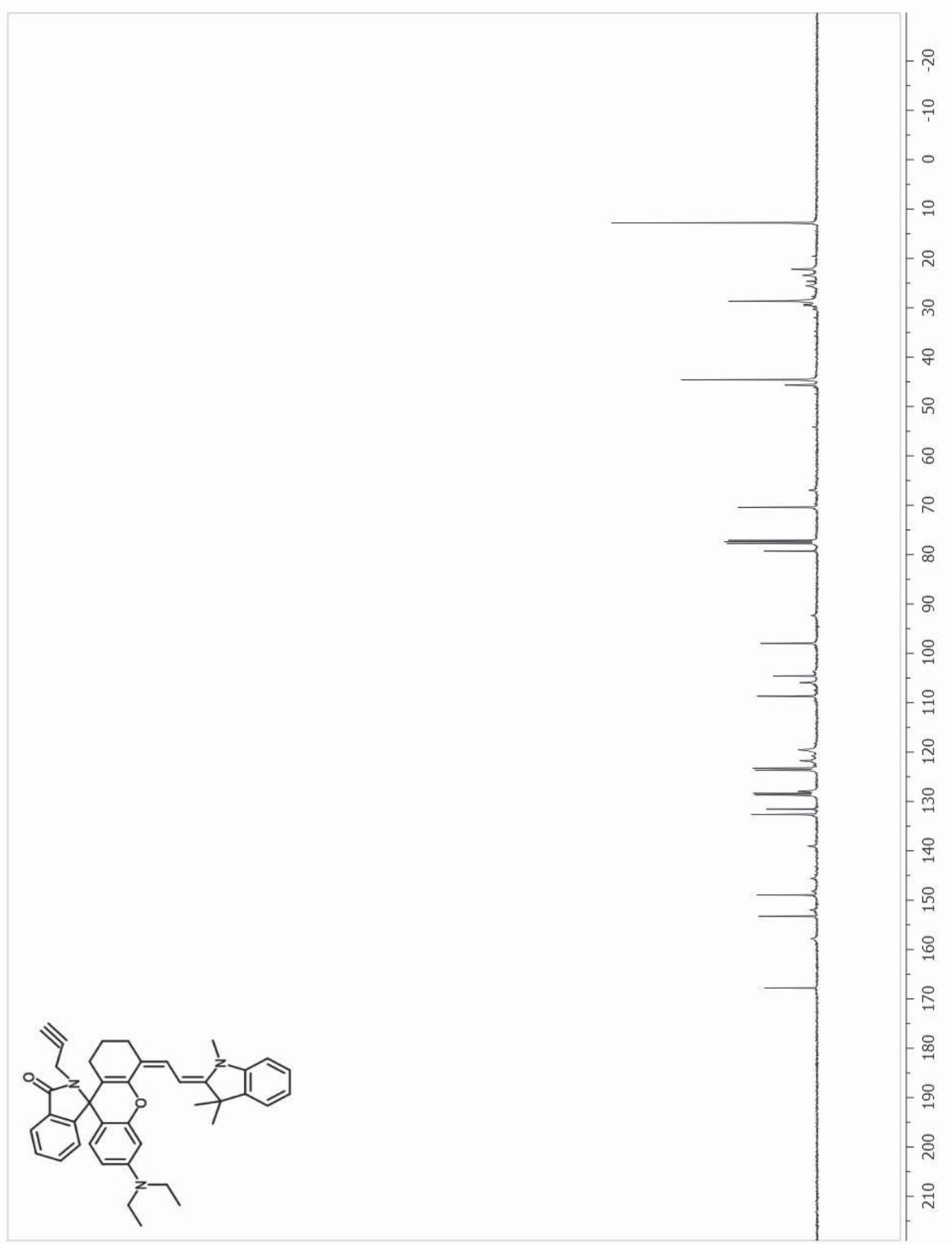

Figure B.10. ${ }^{13} \mathrm{C}$ NMR spectrum of compound $\mathbf{C}$ 


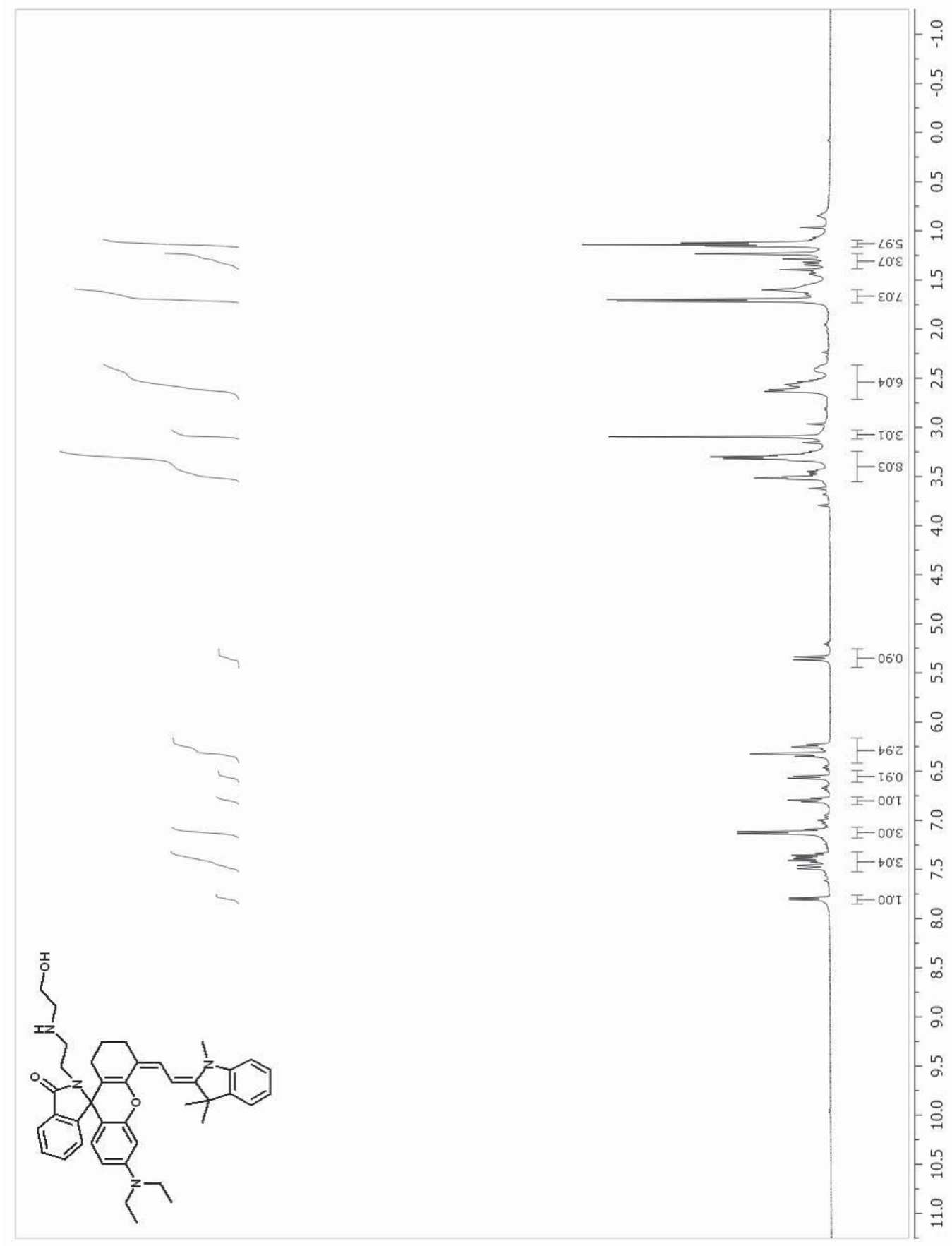

Figure B.11. ${ }^{1} \mathrm{H}$ NMR spectrum of compound D 


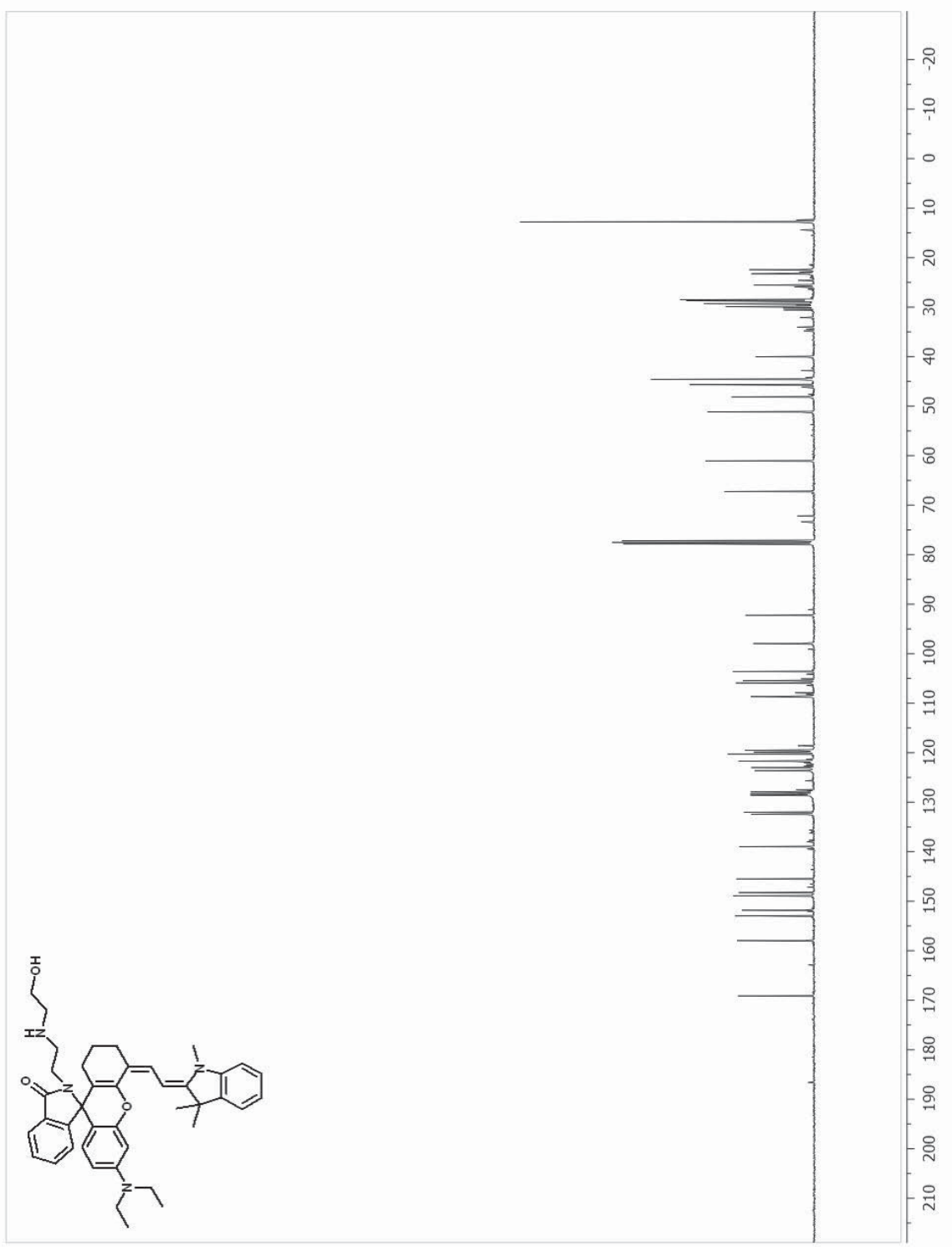

Figure B.12. ${ }^{13} \mathrm{C}$ NMR spectrum of compound D 


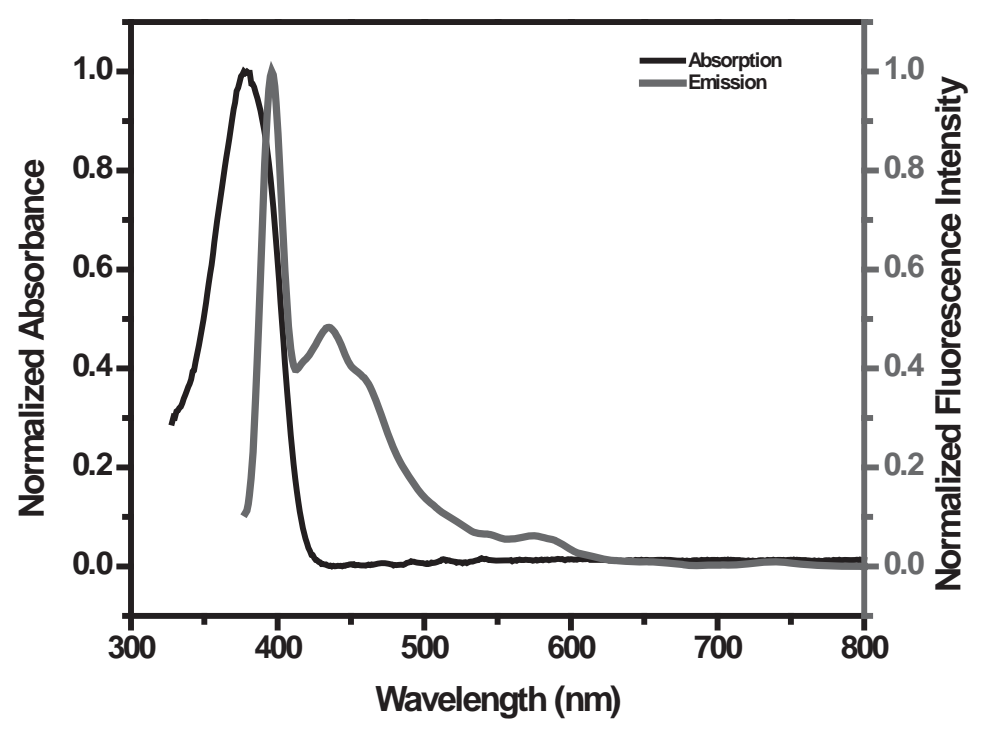

Figure B.13. Normalized absorbance and fluorescence spectra of fluorescent probe $\mathbf{A}$ in EtOH/pH8.0 HEPES Buffer (v/v = 2/3). Excitation wavelength: $350 \mathrm{~nm}$.

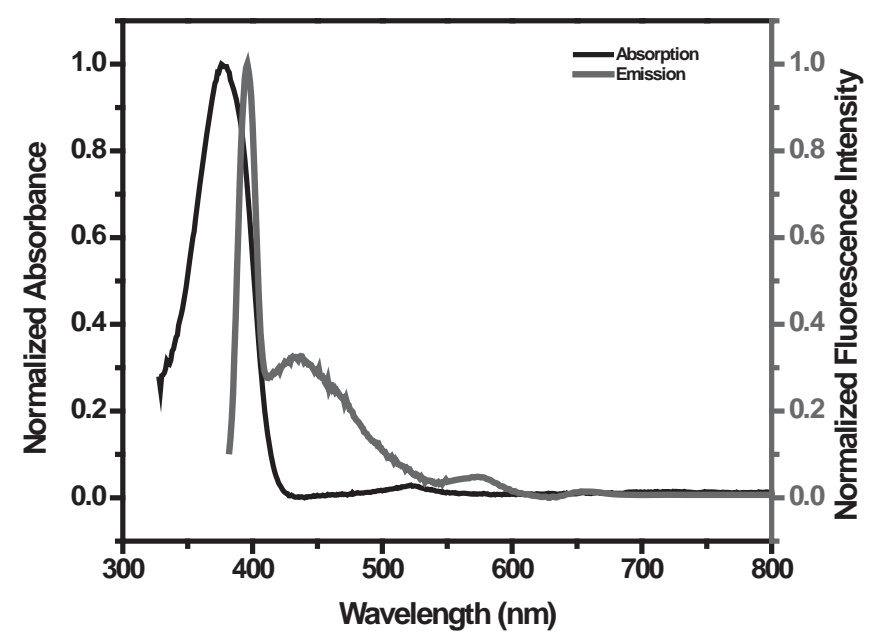

Figure B.14. Normalized absorbance and fluorescence spectra of fluorescent probe $\mathbf{B}$ in EtOH/pH7.0 HEPES Buffer (v/v = 2/3). Excitation wavelength: $350 \mathrm{~nm}$ 


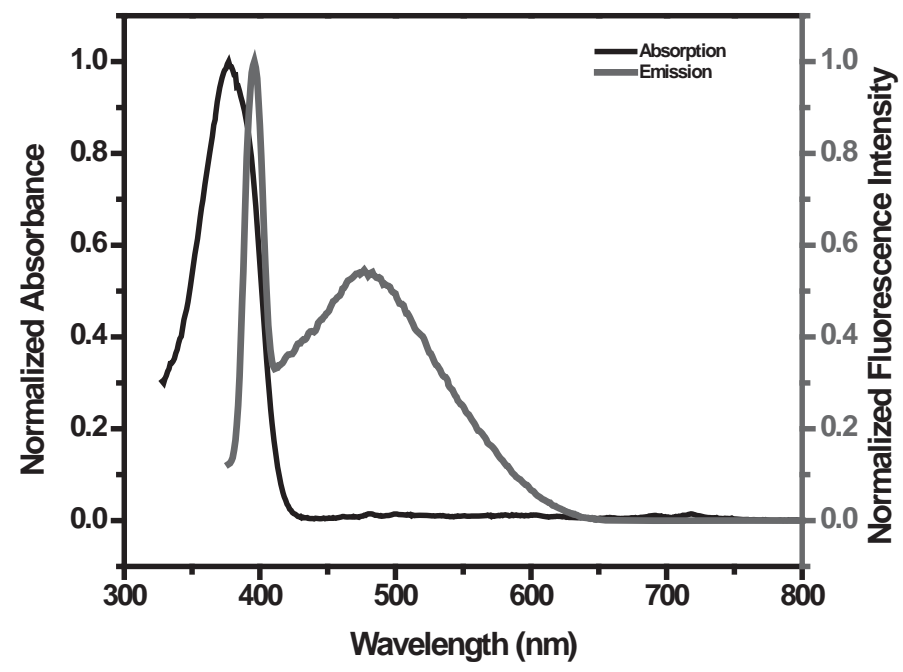

Figure B.15. Normalized absorbance and fluorescence spectra of fluorescent probe $\mathbf{C}$ in $\mathrm{EtOH} / \mathrm{pH}$ 7.0 HEPES Buffer (v/v = 2/3). Excitation wavelength: $350 \mathrm{~nm}$.

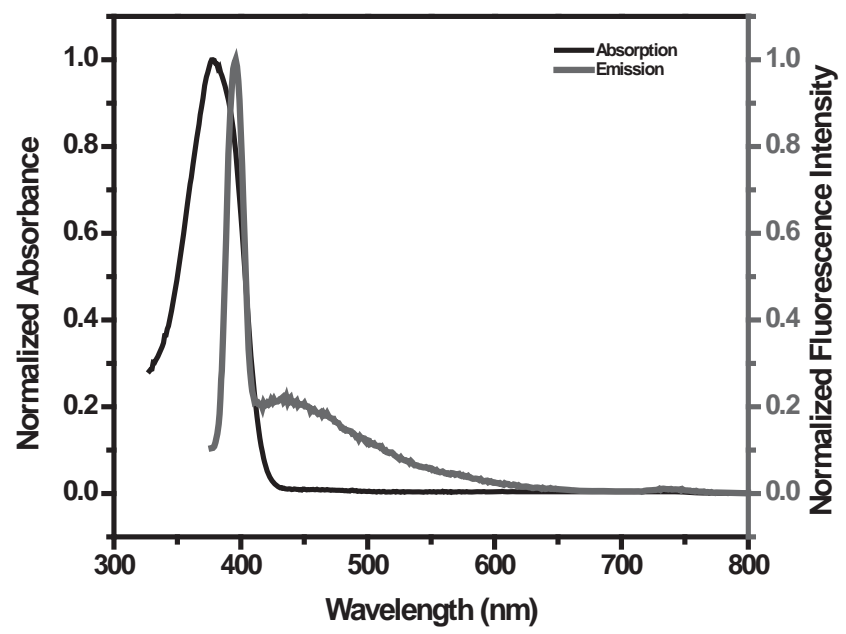

Figure B.16. Normalized absorbance and fluorescence spectra of fluorescent probe $\mathbf{D}$ in $\mathrm{EtOH} / \mathrm{pH}$.0 HEPES Buffer (v/v = 2/3). Excitation wavelength: $350 \mathrm{~nm}$ 

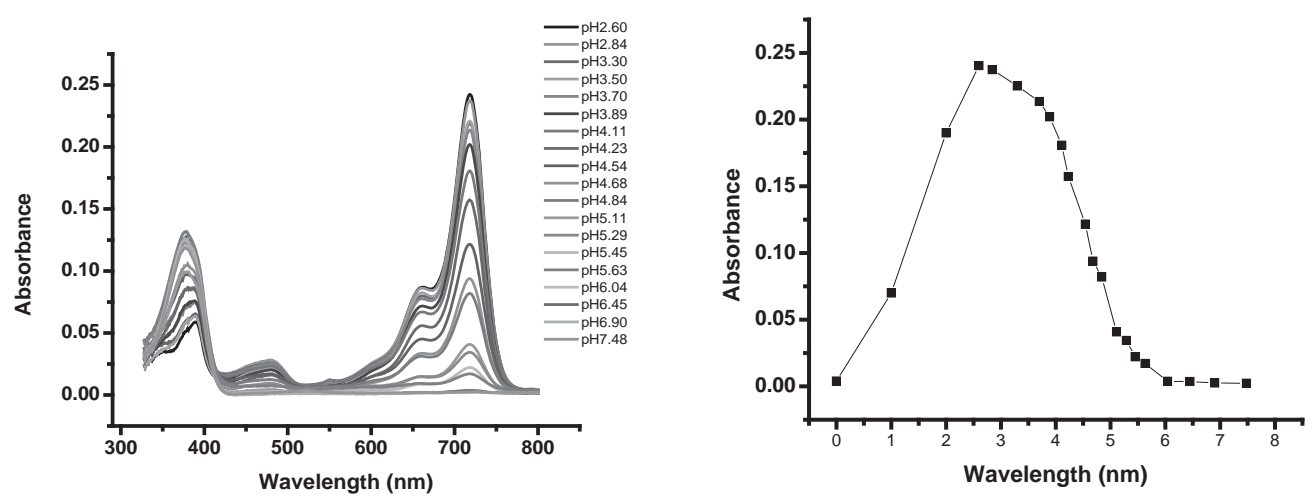

Figure B.17. Absorption spectra of $5 \mu \mathrm{M}$ fluorescent probe $\mathbf{B}$ at different $\mathrm{pH}$ values (left) and effect of $\mathrm{pH}$ on absorbance of the fluorescent probe $\mathbf{B}$ at $718 \mathrm{~nm}$ (right).

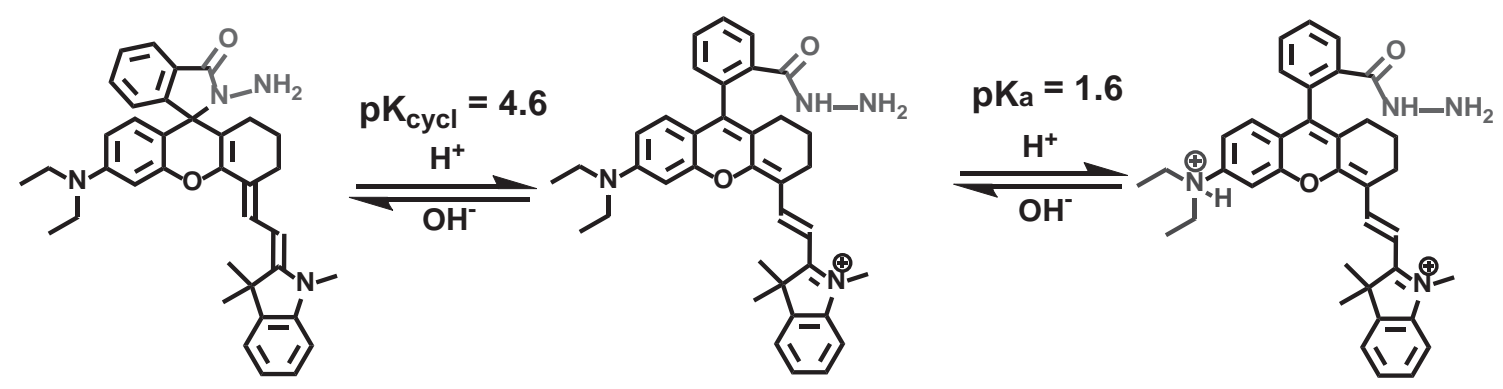

Fluorescent probe B
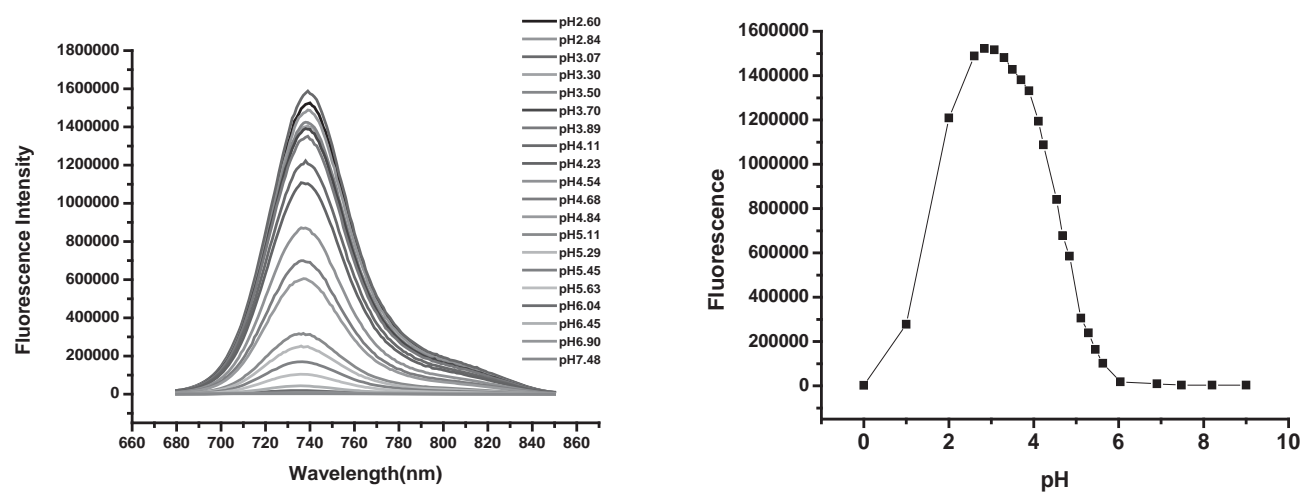

Figure B.18. Fluorescence spectra of $5 \mu \mathrm{M}$ fluorescent probe $\mathbf{B}$ at different $\mathrm{pH}$ values (left) and effect of $\mathrm{pH}$ on fluorescence of the fluorescent probe $\mathbf{B}$ at $743 \mathrm{~nm}$ (right). Excitation wavelength: $670 \mathrm{~nm}$ 

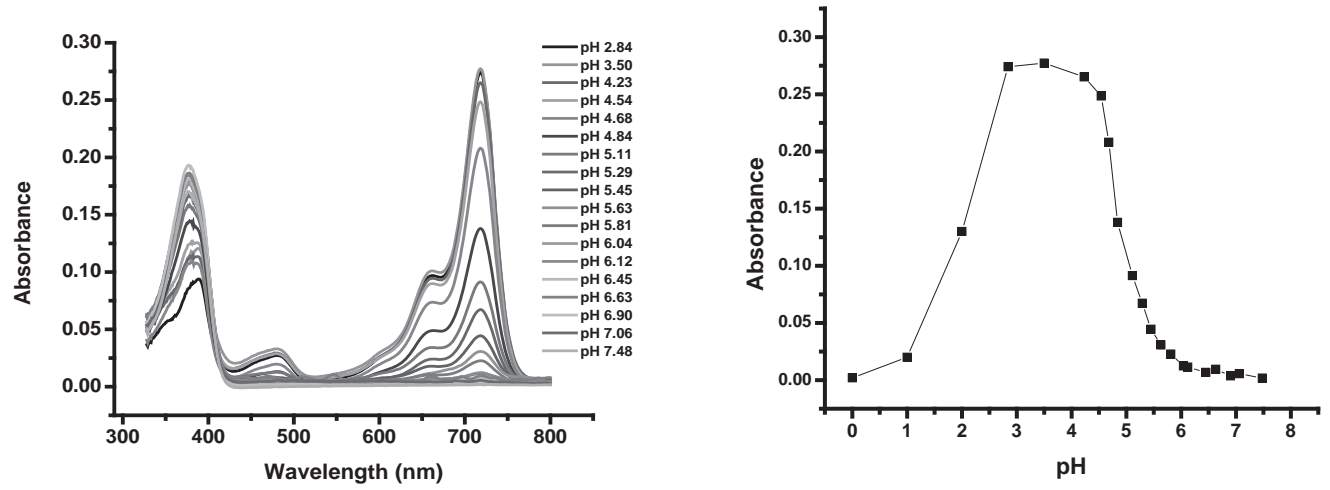

Figure B.19. Absorption spectra of $5 \mu \mathrm{M}$ fluorescent probe $\mathbf{C}$ at different $\mathrm{pH}$ values (left) and effect of $\mathrm{pH}$ on absorbance of the fluorescent probe $\mathbf{C}$ at $718 \mathrm{~nm}$ (right).

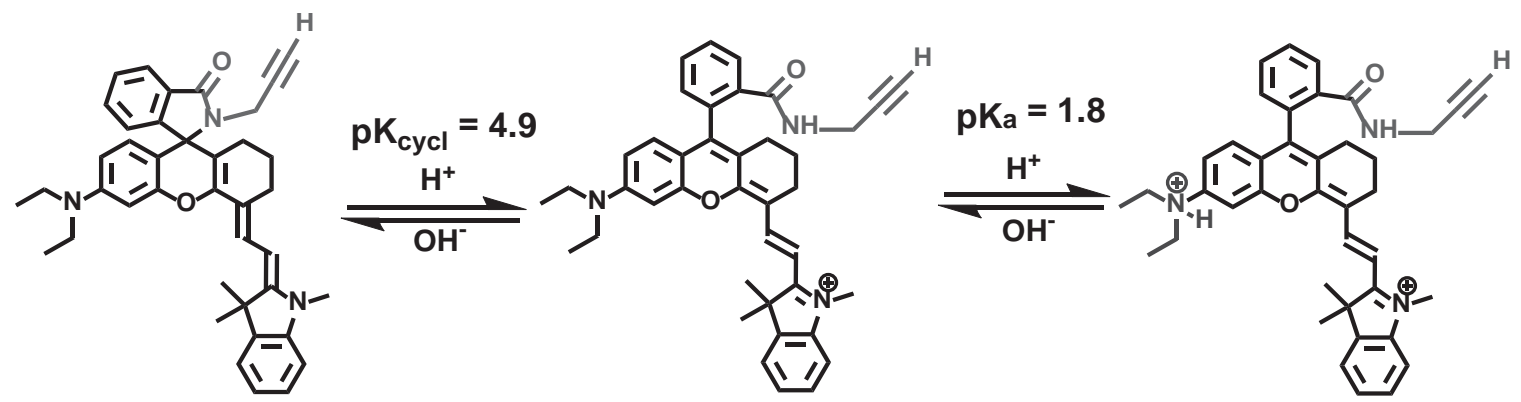

Fluorescent probe $\mathrm{C}$
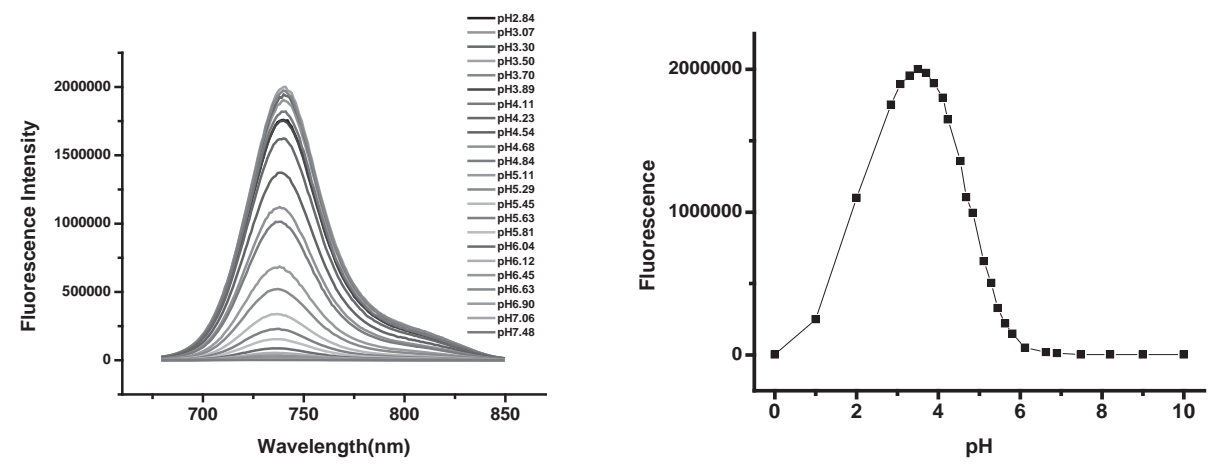

Figure B.20. Fluorescence spectra of $5 \mu \mathrm{M}$ fluorescent probe $\mathbf{C}$ at different $\mathrm{pH}$ values (left) and effect of $\mathrm{pH}$ on fluorescence of the fluorescent probe $\mathbf{C}$ at $743 \mathrm{~nm}$ (right). Excitation wavelength: $670 \mathrm{~nm}$. 


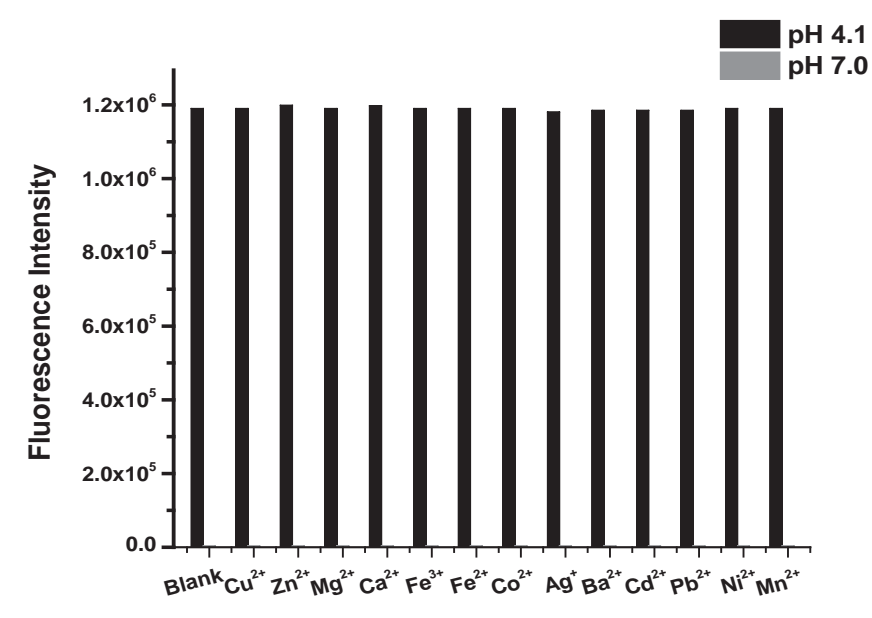

Figure B.21. Fluorescent responses of $5 \mu \mathrm{M}$ fluorescent probe $\mathbf{B}$ to different metal ions $(200 \mu \mathrm{M})$ at $\mathrm{pH} 4.1$ and 7.0. Excitation wavelength: $670 \mathrm{~nm}$.

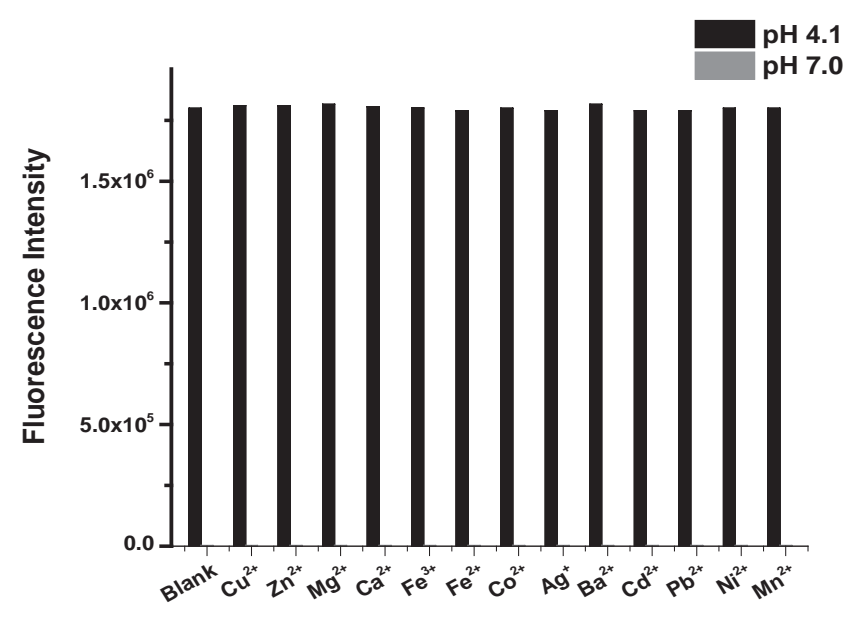

Figure B.22. Fluorescent responses of $5 \mu \mathrm{M}$ fluorescent probe $\mathbf{C}$ to different metal ions $(200 \mu \mathrm{M})$ at $\mathrm{pH} 4.1$ and 7.0. Excitation wavelength: $670 \mathrm{~nm}$. 


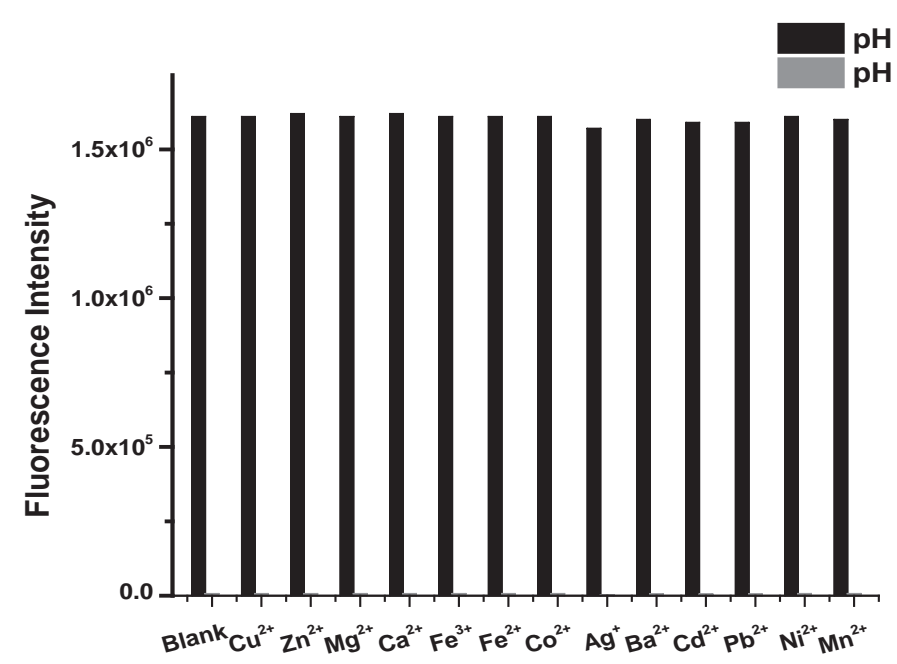

Figure B.23. Fluorescent responses of $5 \mu \mathrm{M}$ fluorescent probe $\mathbf{D}$ to different metal ions $(200 \mu \mathrm{M})$ at $\mathrm{pH} 4.1$ and 7.0. Excitation wavelength: $670 \mathrm{~nm}$.

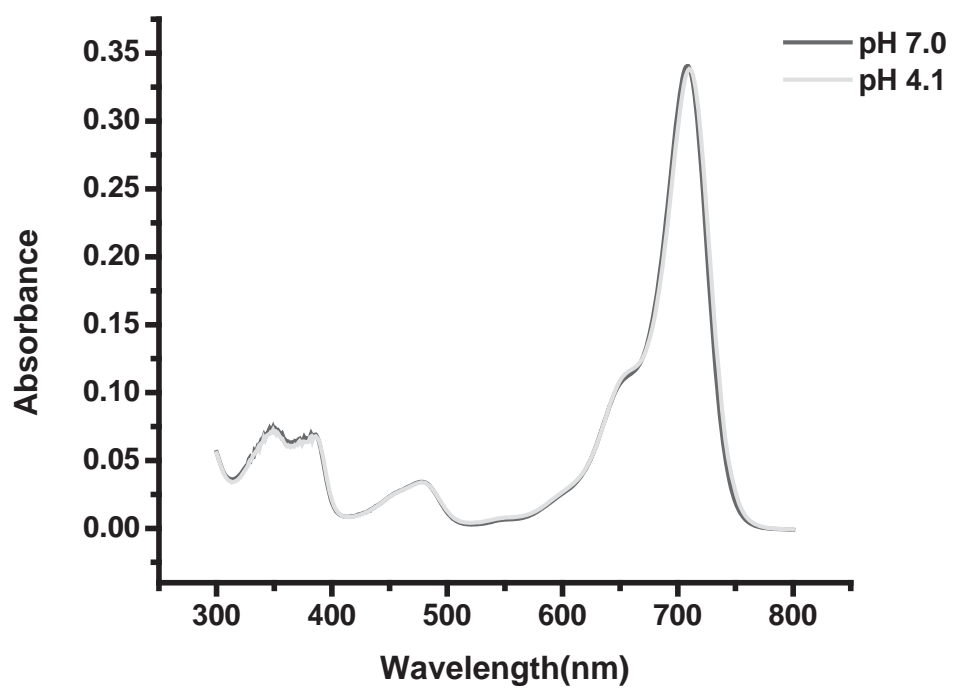

Figure B.24. Absorbance spectra of compound $\mathbf{5}$ at $\mathrm{pH} 4.1$ and 7.0. 


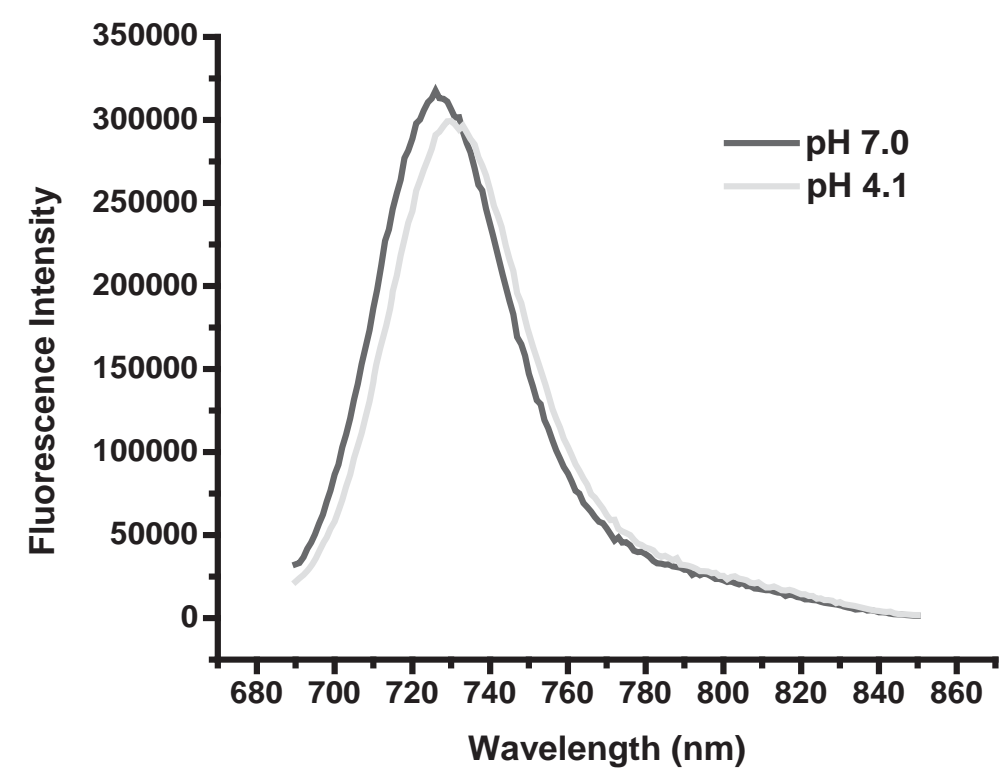

Figure B.25. Fluorescence spectra of compound $\mathbf{5}$ at $\mathrm{pH} 4.1$ and 7.0.

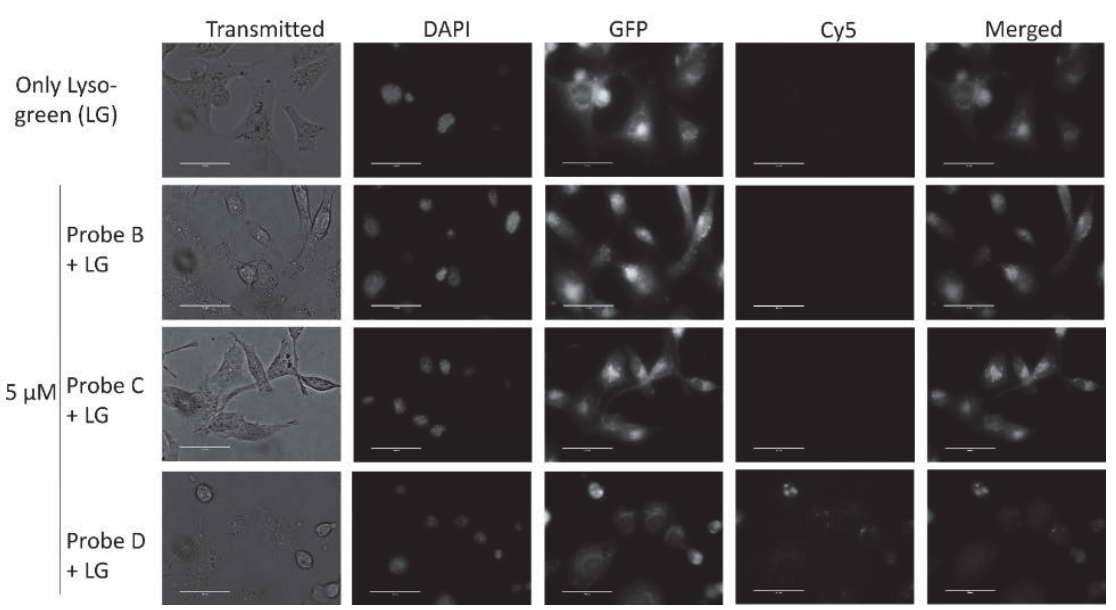

Figure B.26. Fluorescence images of MDA-MB-231 cells incubated with fluorescent probes B, C, and D. Cells were incubated with $5 \mu \mathrm{M}$ of fluorescent probes $\mathbf{B}, \mathbf{C}$, and $\mathbf{D}$ for $2 \mathrm{~h}$ and imaged for co-localization in presence of LysoSensor Green, a lysosomal stain and Hoechest, a nuclear stain. The images were acquired using inverted fluorescence microscope at 60X magnification. 
Table B.1: Absorption coefficients and $\mathrm{pK}_{\mathrm{a}}$ values of the probes

\begin{tabular}{|c|c|c|}
\hline Compound & $\begin{array}{c}\text { Absorption } \\
\text { coefficient (EtOH) }\end{array}$ & $\begin{array}{c}\mathbf{p K}_{\mathbf{a}} \text {, pK } \mathbf{c y c l} \\
\text { (Citrate-phosphate } \\
\text { buffer with } \\
\mathbf{4 0 \%} \mathbf{E t O H})\end{array}$ \\
\hline A & $9.8 \times 10^{4}$ & $1.56,5.80$ \\
\hline B & $5.2 \times 10^{4}$ & $1.55,4.60$ \\
\hline C & $6.2 \times 10^{4}$ & $1.81,4.86$ \\
\hline D & $1.1 \times 10^{5}$ & $1.56,5.38$ \\
\hline $\mathbf{5}$ & $8.8 \times 10^{4}$ & \\
\hline
\end{tabular}




\section{APPENDIX C \\ SUPPORTING INFORMSTION FOR CHAPTER 4 \\ Synthesis of a Hybrid Dye, Tested its Reactivity for Fluorescent Probe \\ Design}

Giri Vegesna, ${ }^{\mathrm{a}}$ Jianheng Bi, ${ }^{\mathrm{a}}$ Fen-Tair Luo ${ }^{\mathrm{b} *}$, and Haiying Liu ${ }^{\mathrm{a} *}$

${ }^{a}$ Department of Chemistry, Michigan Technological University, Houghton, MI 49931

${ }^{b}$ Institute of Chemistry, Academia Sinica, Taipei, Taiwan 11529, Republic of China

\section{Table of Contents}

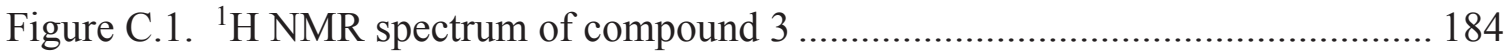

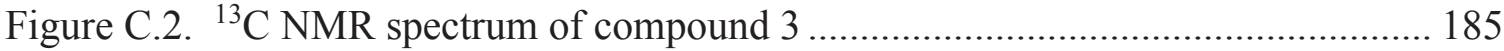

Figure C.3. ${ }^{1} \mathrm{H}$ NMR spectrum of compound 4 ………............................................ 186

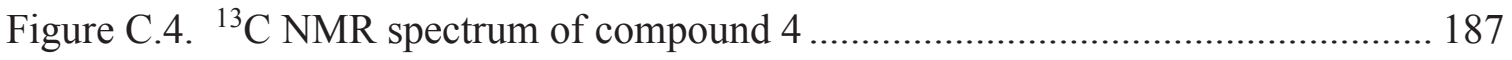

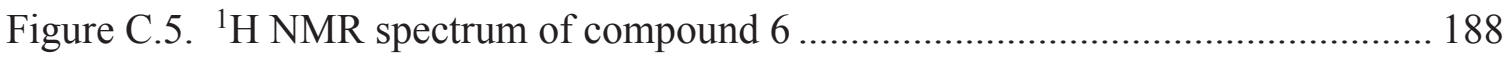

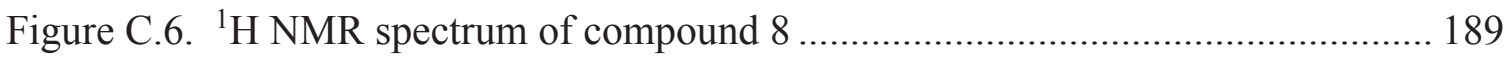

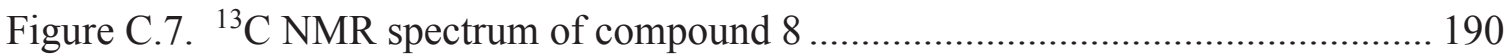

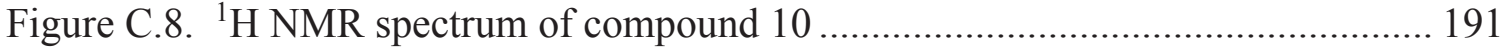

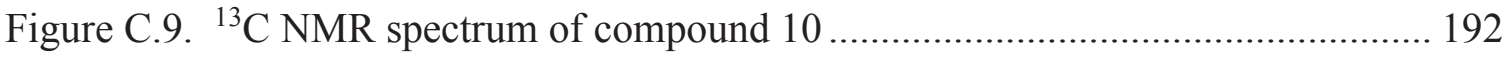

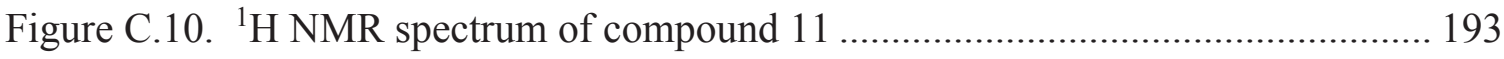

Figure C.11. ${ }^{13} \mathrm{C}$ NMR spectrum of compound 11 ..................................................... 194

Figure C.12. ${ }^{1} \mathrm{H}$ NMR spectrum of compound 12 .................................................... 195

Figure C.13. ${ }^{13} \mathrm{C}$ NMR spectrum of compound 11 .................................................... 196

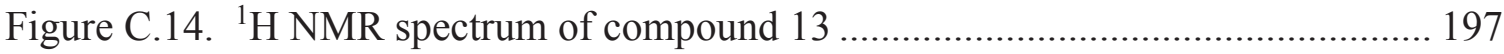

Figure C.15. ${ }^{13} \mathrm{C}$ NMR spectrum of compound 13 .................................................... 198

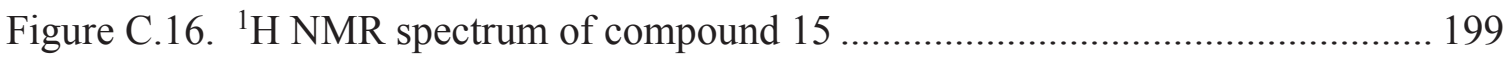

Figure C.17. ${ }^{13} \mathrm{C}$ NMR spectrum of compound 15 ................................................... 200 


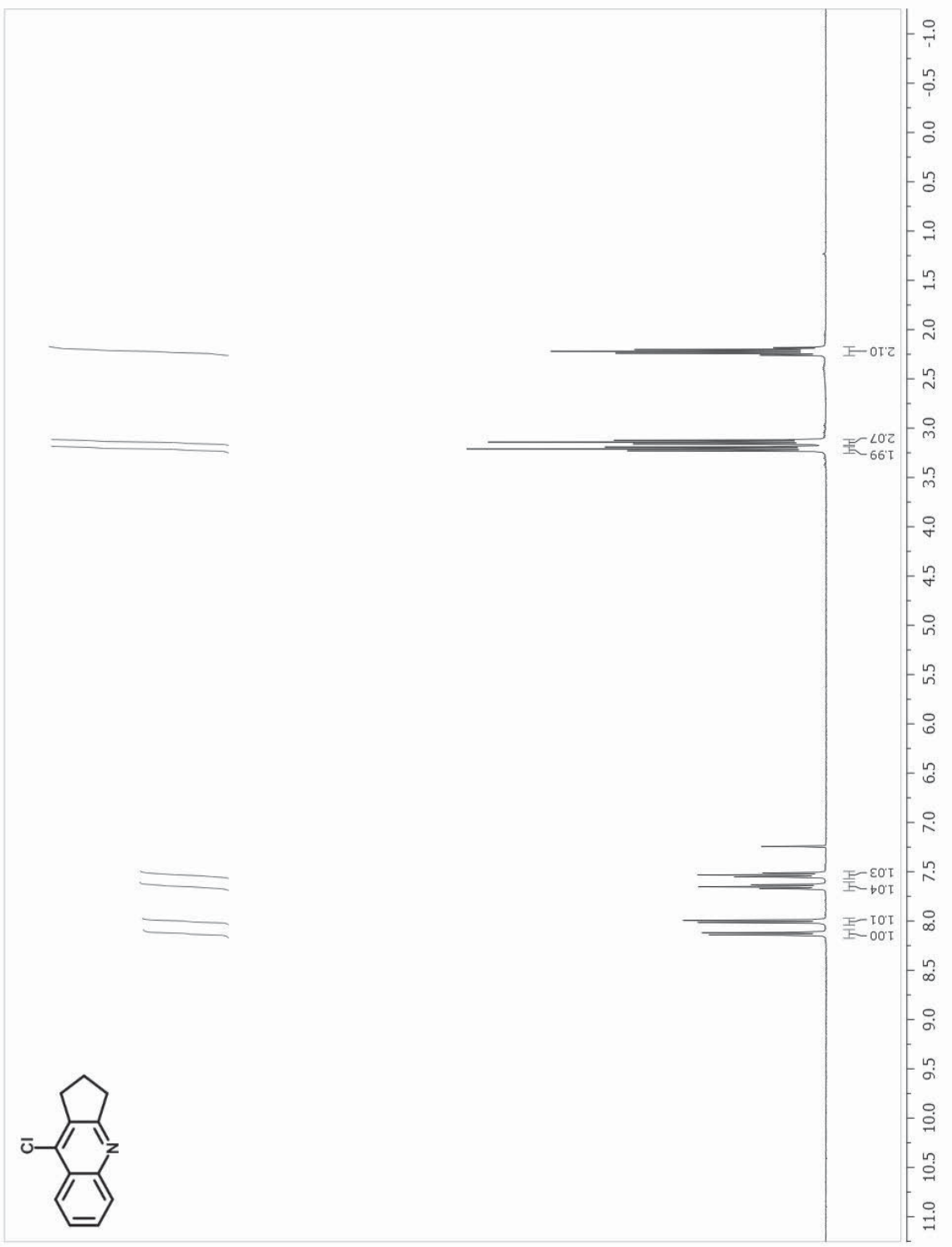

Figure C.1. ${ }^{1} \mathrm{H}$ NMR spectrum of compound 3 


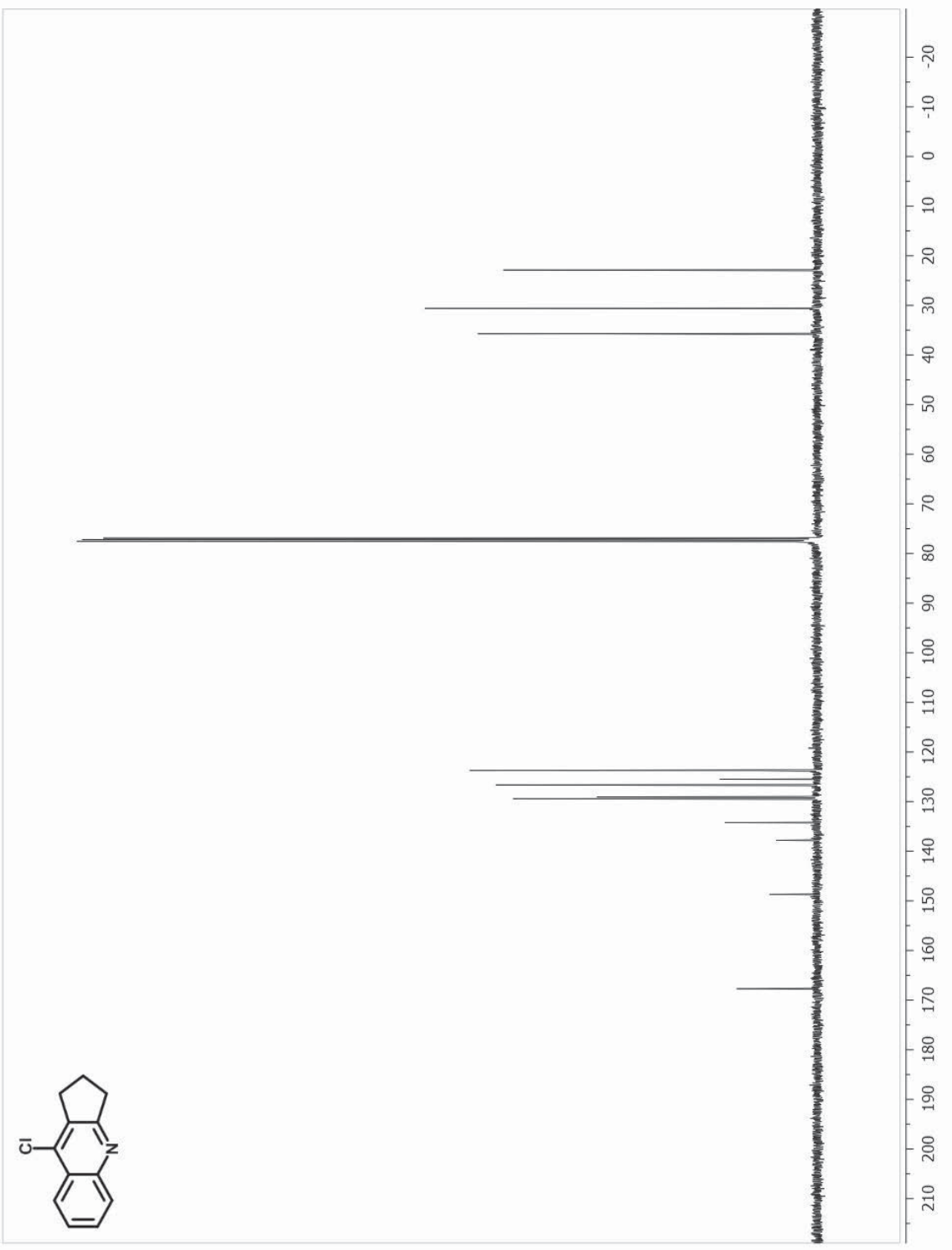

Figure C.2. ${ }^{13} \mathrm{C}$ NMR spectrum of compound $\mathbf{3}$ 


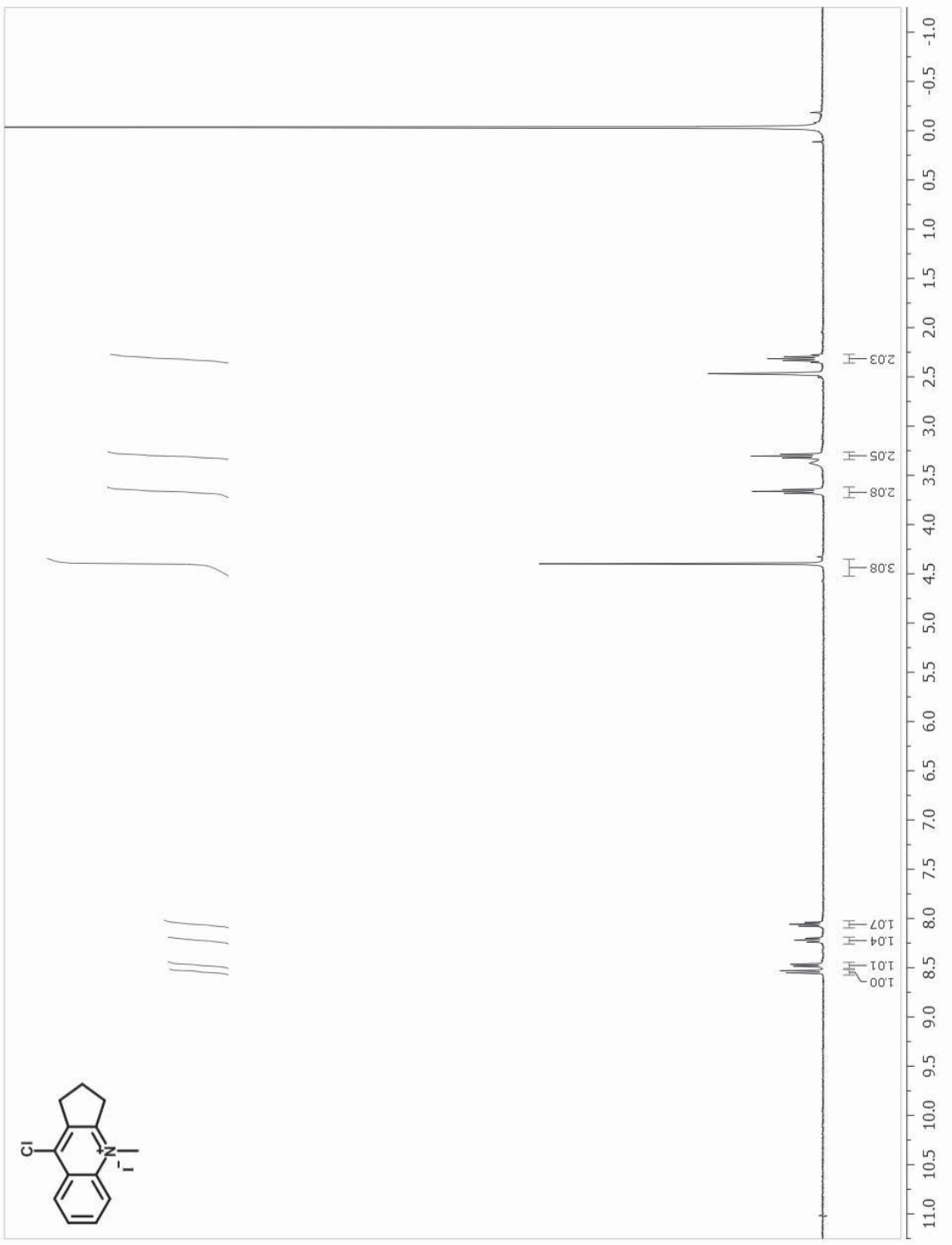

Figure C.3. ${ }^{1} \mathrm{H}$ NMR spectrum of compound 4 


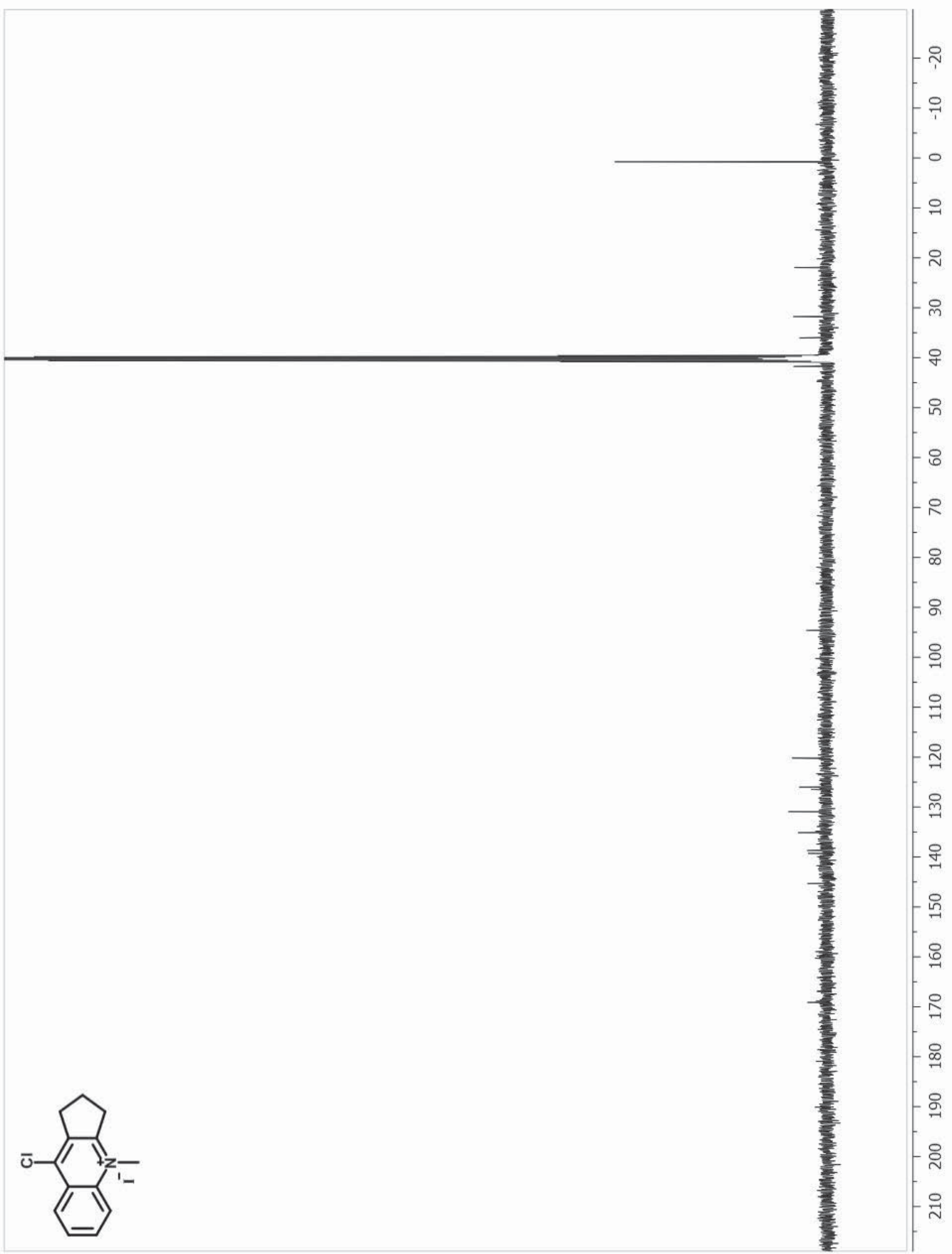

Figure C.4. ${ }^{13} \mathrm{C}$ NMR spectrum of compound 4 


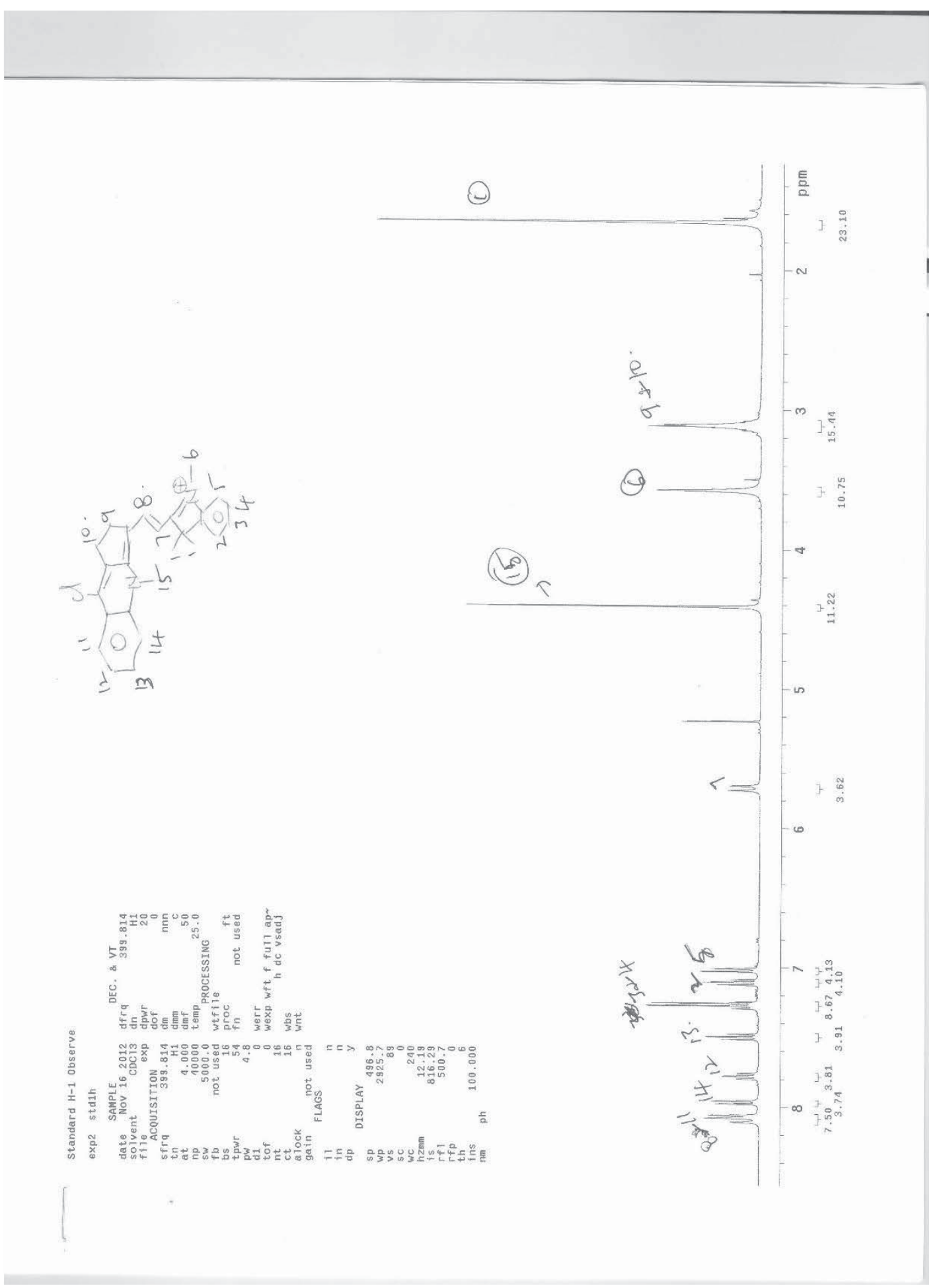

Figure C.5. ${ }^{1} \mathrm{H}$ NMR spectrum of compound 6 


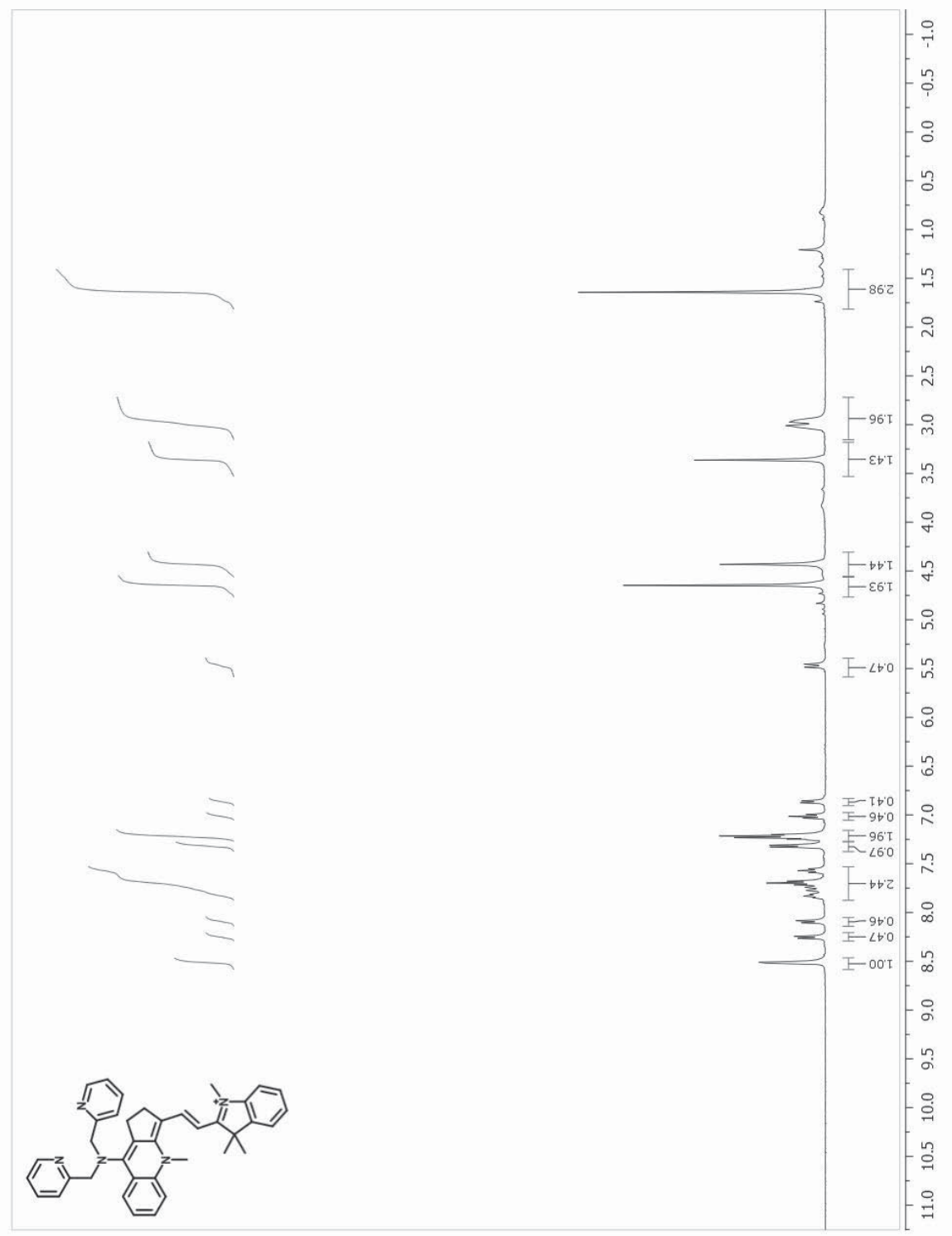

Figure C.6. ${ }^{1}$ H NMR spectrum of compound $\mathbf{8}$ 


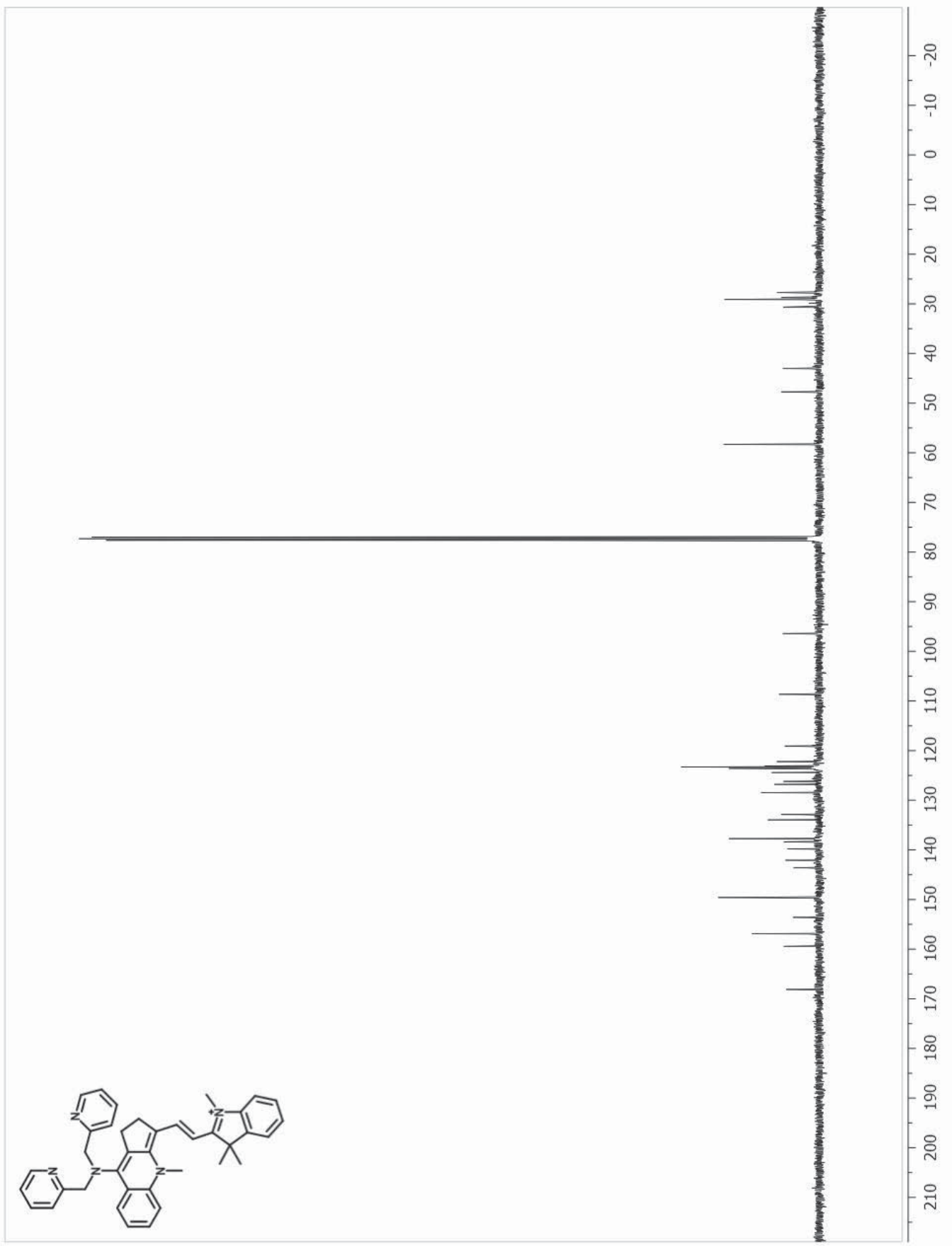

Figure C.7. ${ }^{13} \mathrm{C}$ NMR spectrum of compound $\mathbf{8}$ 


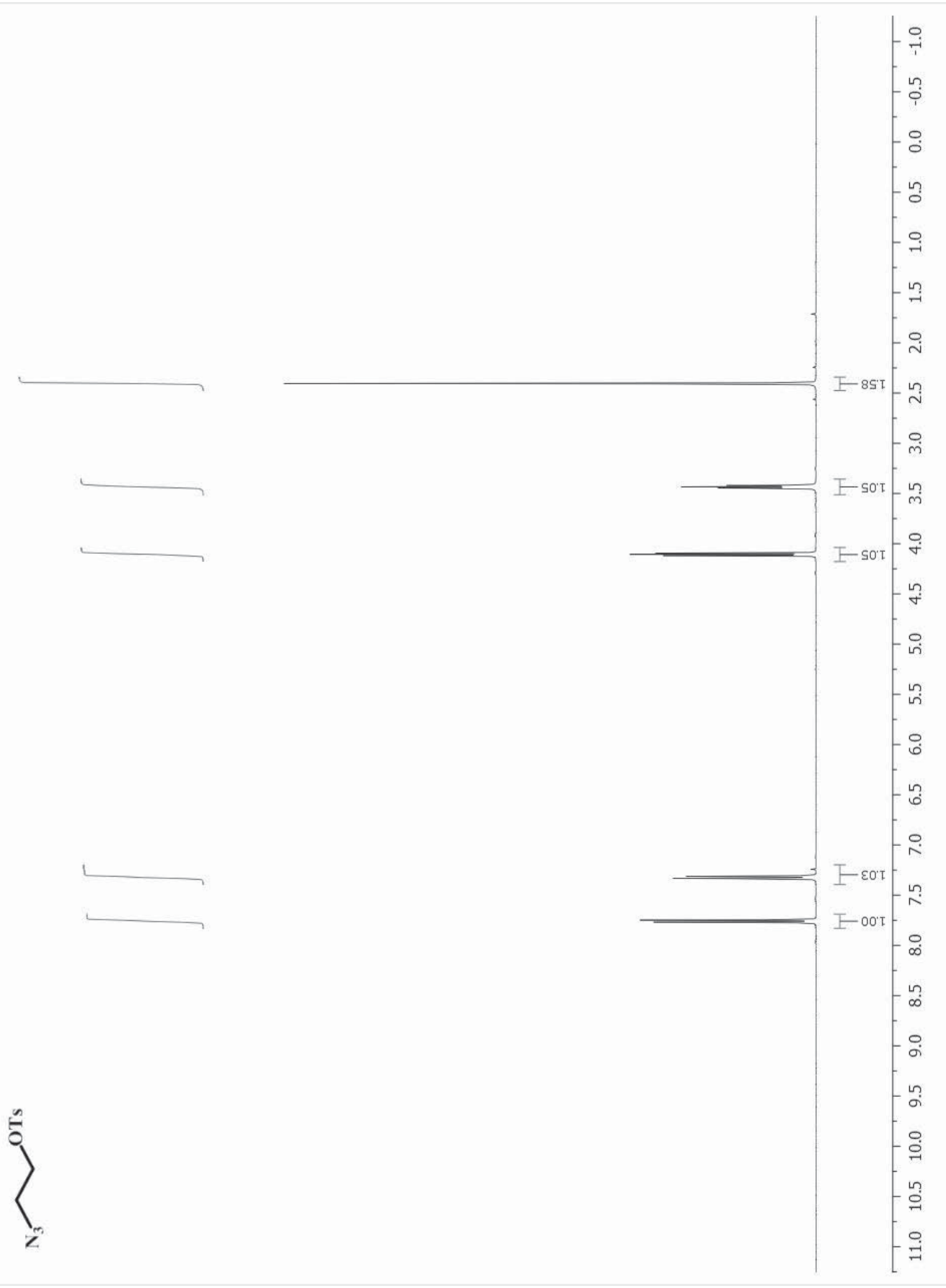

Figure C.8. ${ }^{1} \mathrm{H}$ NMR spectrum of compound $\mathbf{1 0}$ 


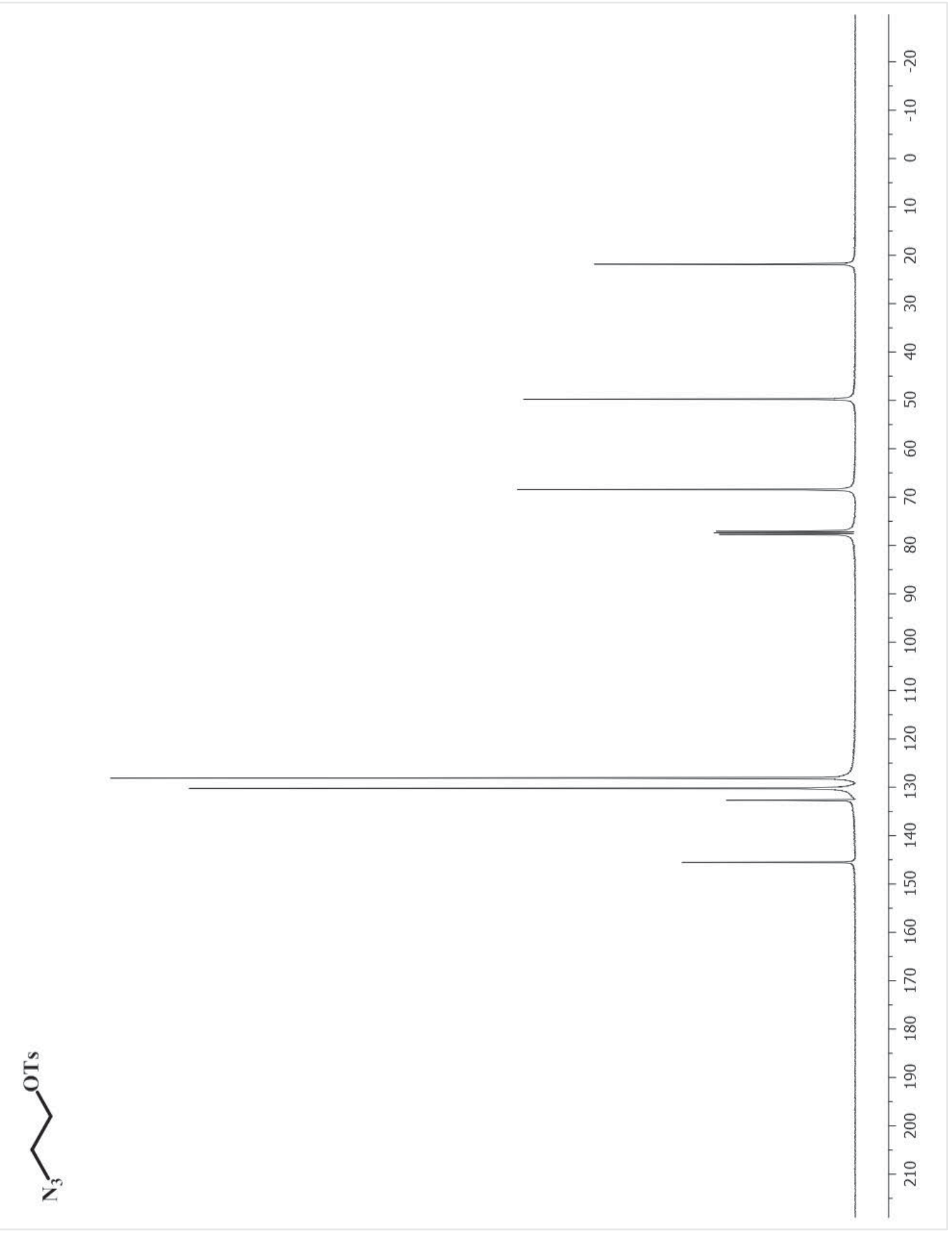

Figure C.9. ${ }^{13} \mathrm{C}$ NMR spectrum of compound $\mathbf{1 0}$ 


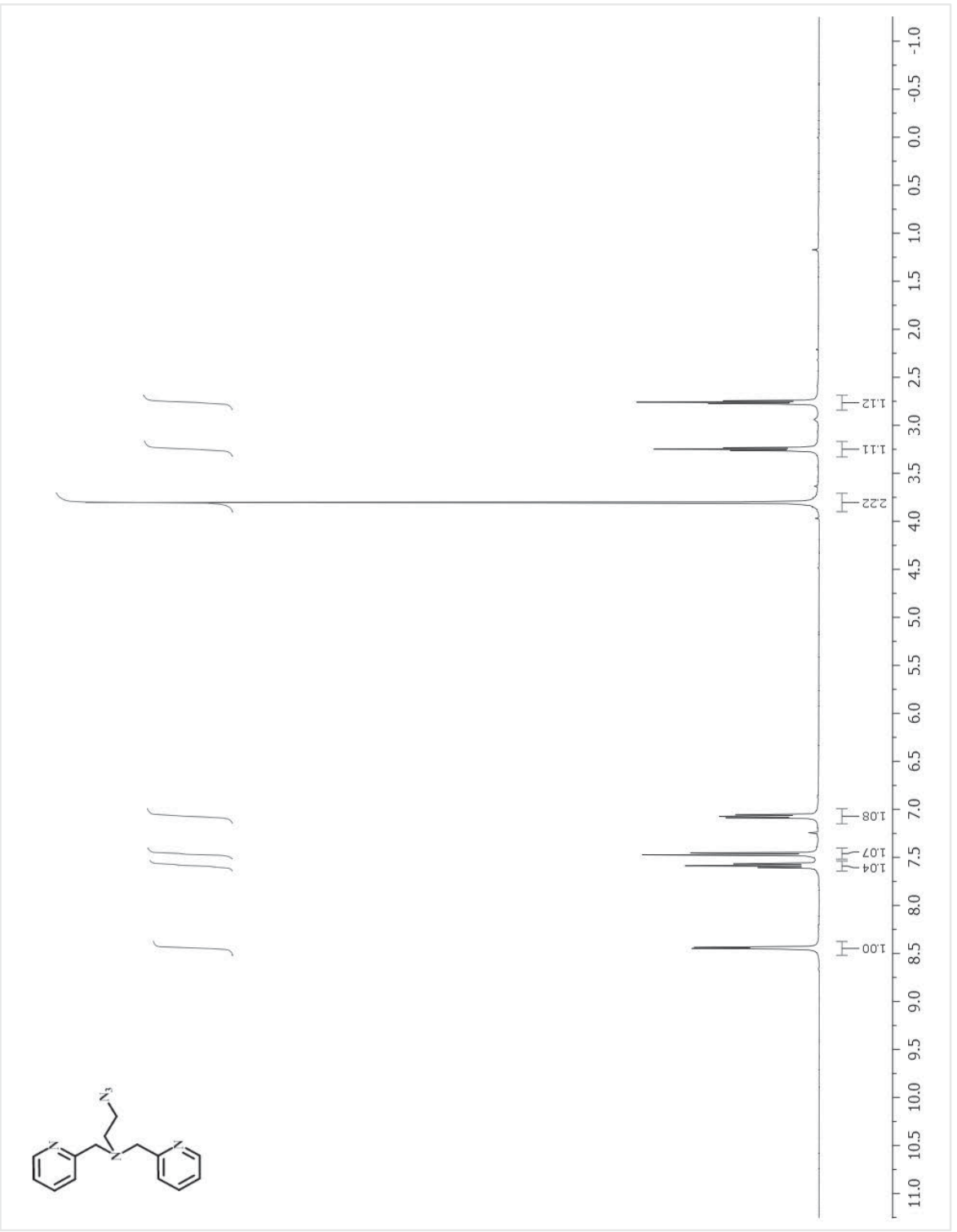

Figure C.10. ${ }^{1} \mathrm{H}$ NMR spectrum of compound $\mathbf{1 1}$ 


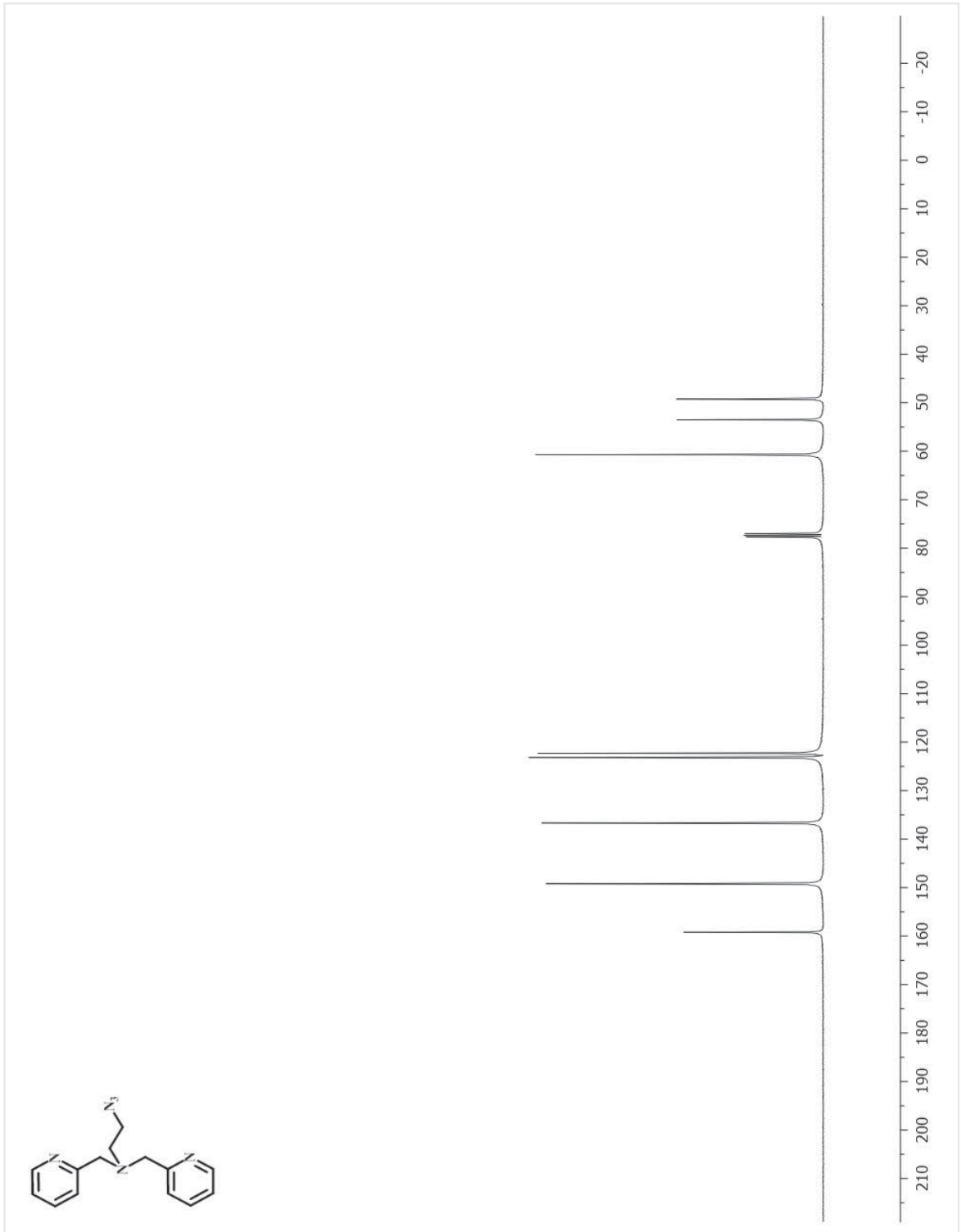

Figure C.11. ${ }^{13} \mathrm{C}$ NMR spectrum of compound $\mathbf{1 1}$ 


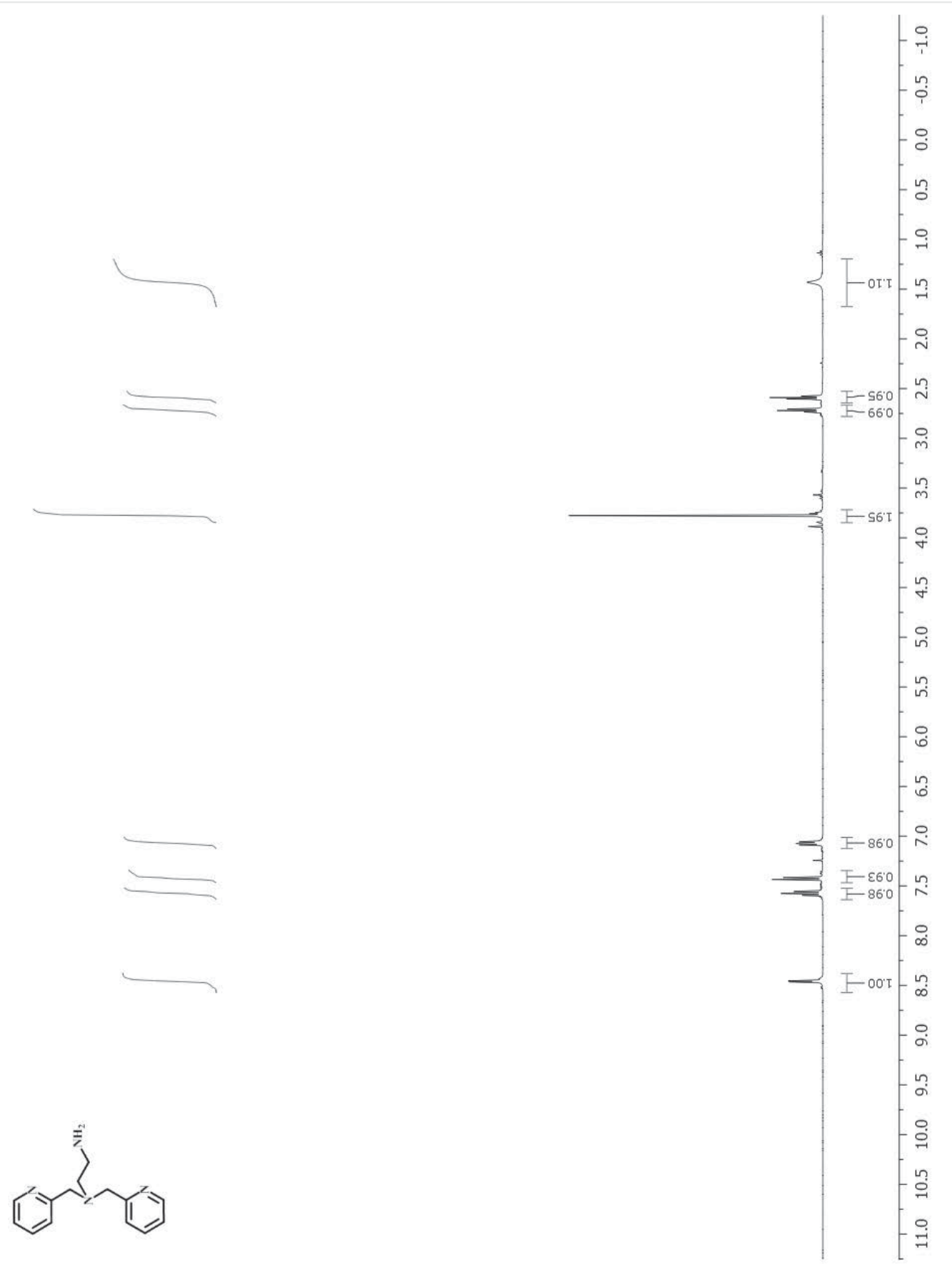

Figure C.12. ${ }^{1} \mathrm{H}$ NMR spectrum of compound $\mathbf{1 2}$ 


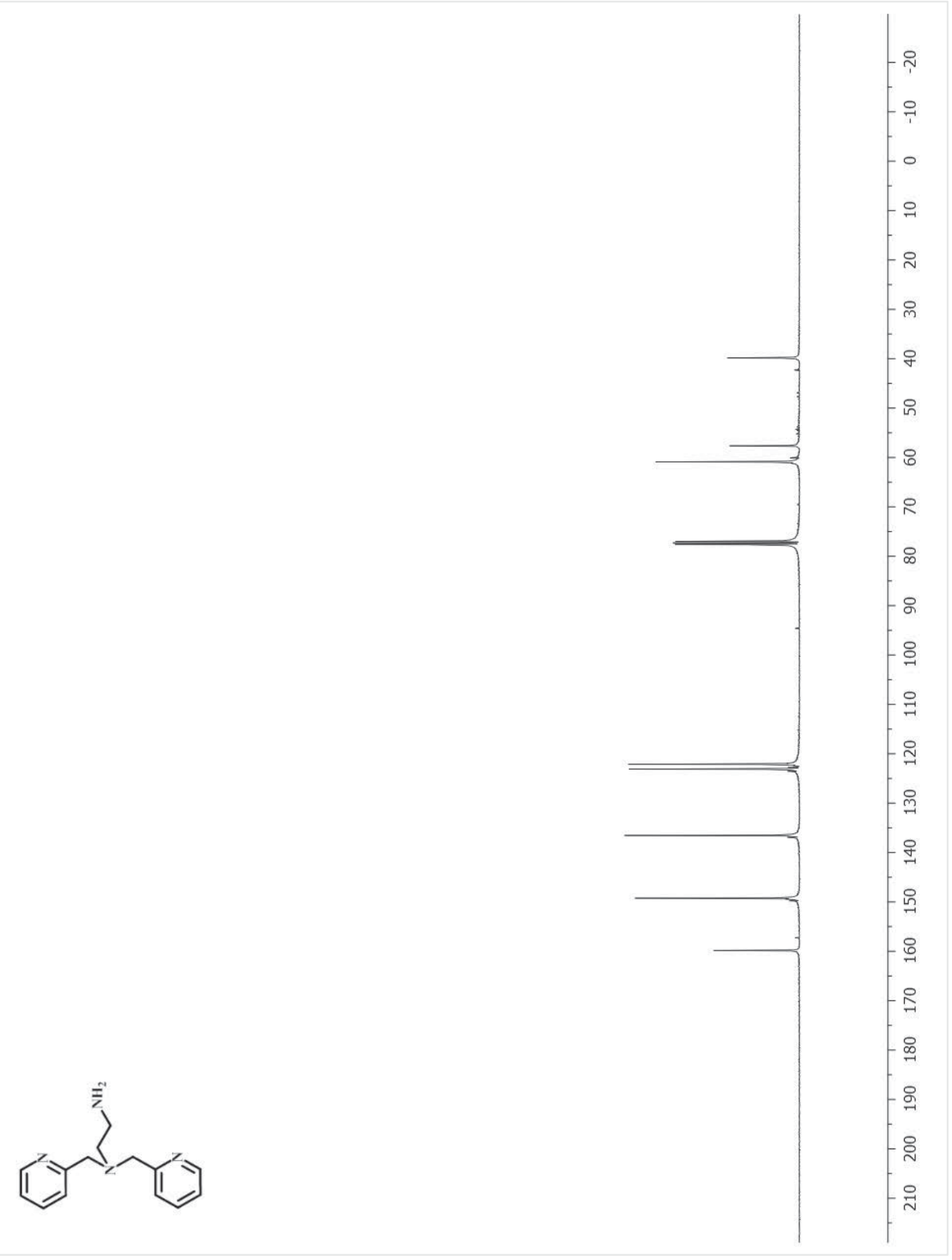

Figure C.13. ${ }^{13} \mathrm{C}$ NMR spectrum of compound $\mathbf{1 1}$ 


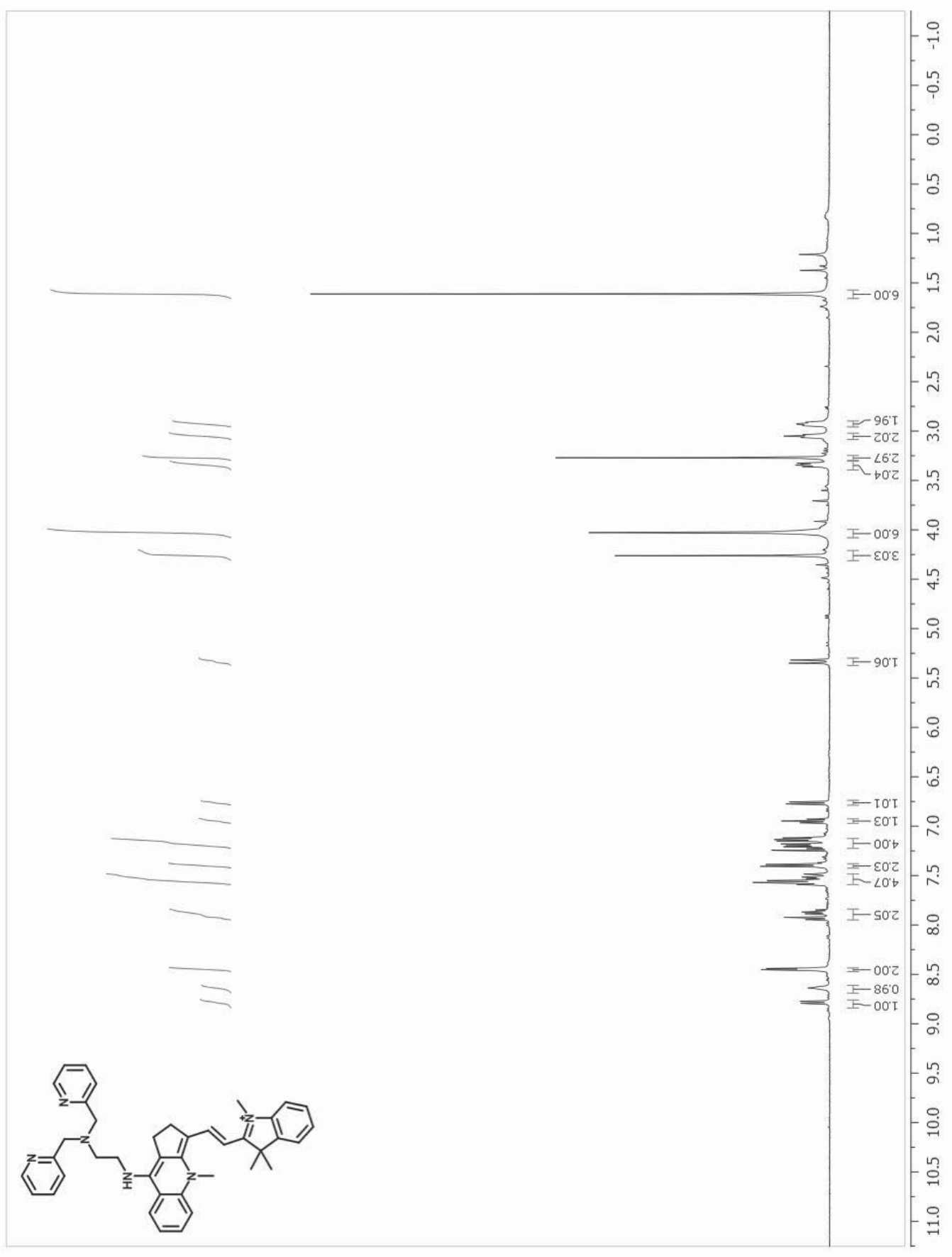

Figure C.14. ${ }^{1} \mathrm{H}$ NMR spectrum of compound $\mathbf{1 3}$ 


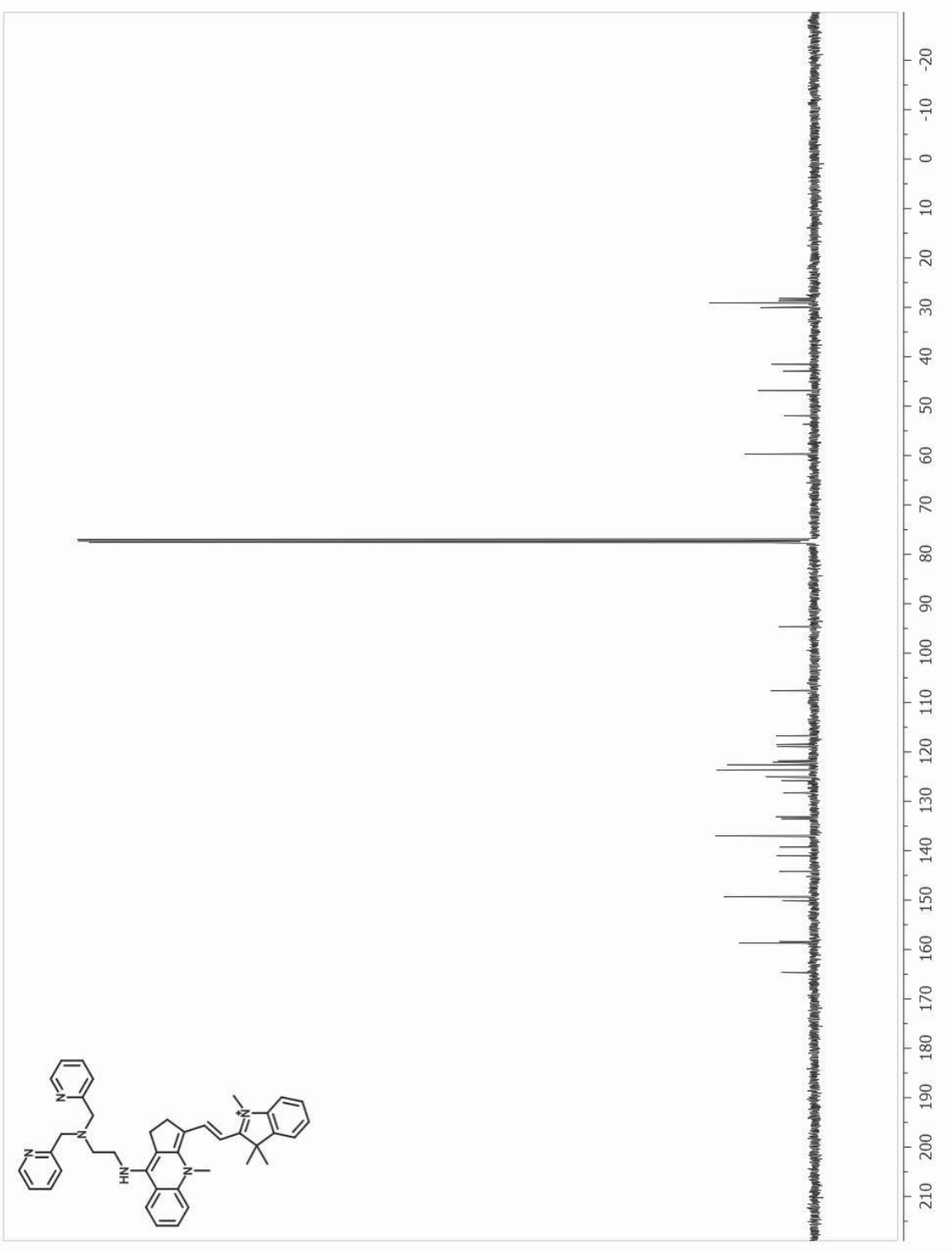

Figure C.15. ${ }^{13} \mathrm{C}$ NMR spectrum of compound $\mathbf{1 3}$ 


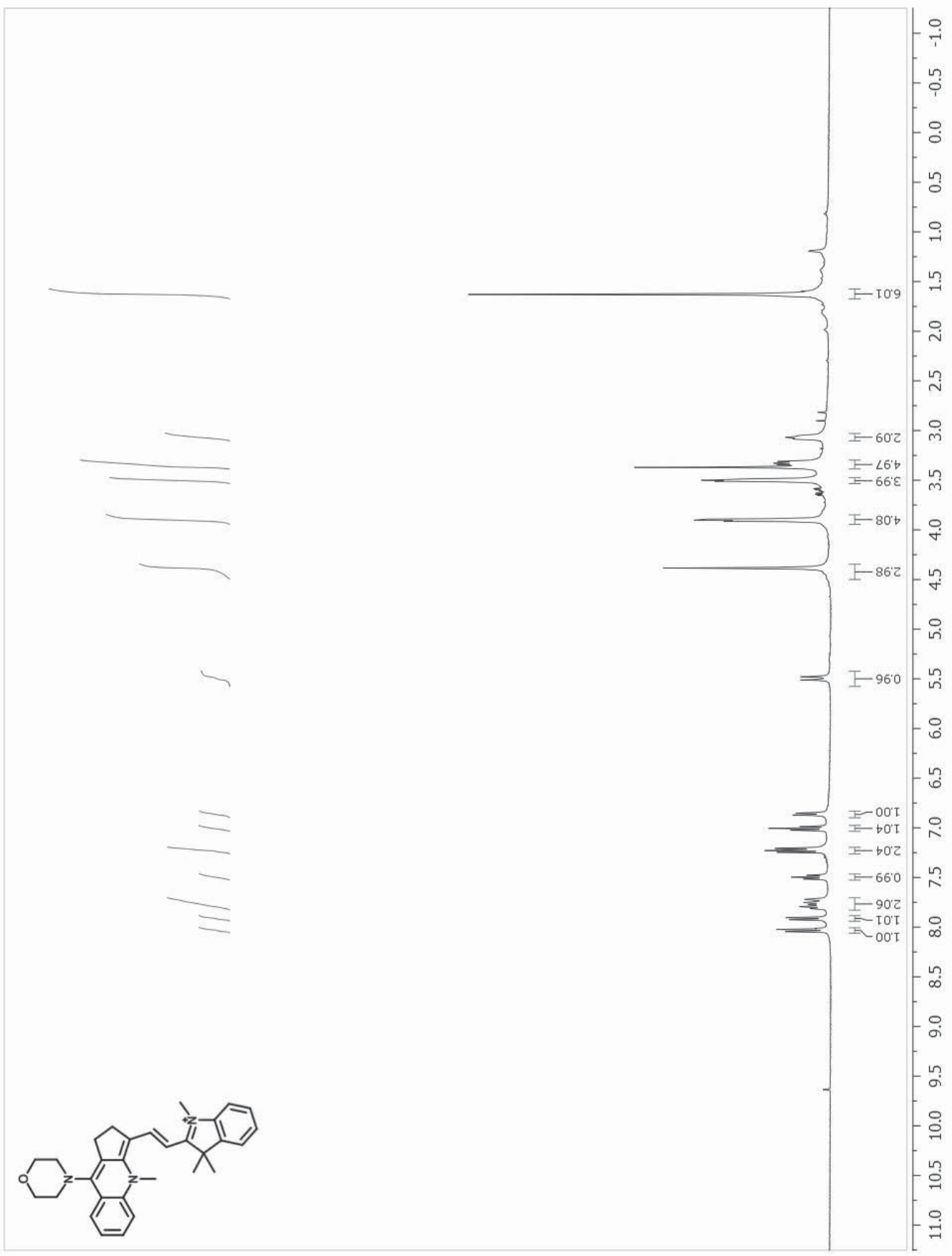

Figure C.16. ${ }^{1} \mathrm{H}$ NMR spectrum of compound $\mathbf{1 5}$ 


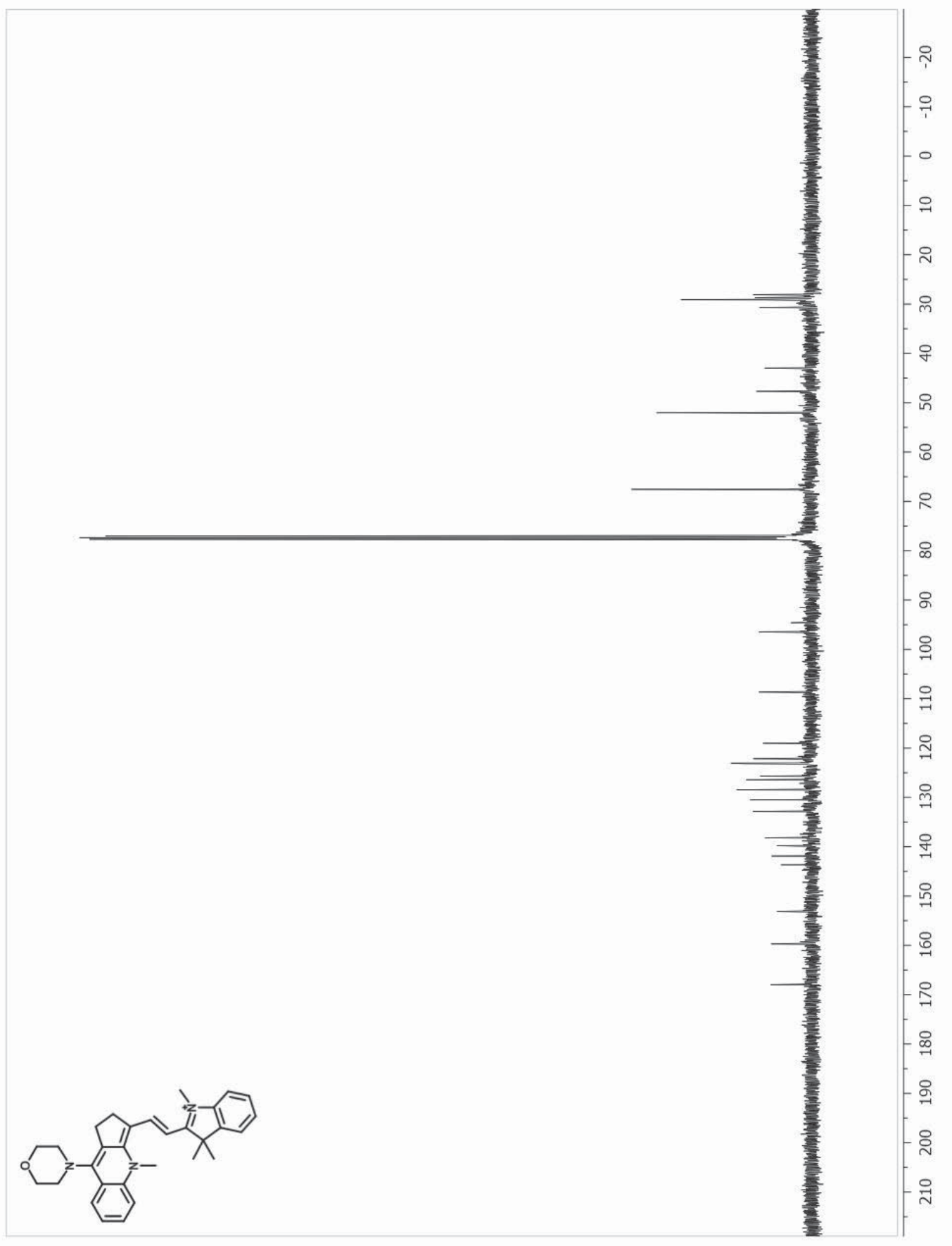

Figure C.17. ${ }^{13} \mathrm{C}$ NMR spectrum of compound $\mathbf{1 5}$ 


\section{APPENDIX D \\ SUPPORTING INFORMATION FOR CHAPTER 5 \\ Glycosylated Aniline Polymer Sensor: Amine to Imine Conversion on Protein-carbohydrate Binding}

Zhe Wanga ${ }^{\mathrm{a}}$ Chunyan Sun ${ }^{\mathrm{a}, \mathrm{b}}$, Giri Vegesna ${ }^{\mathrm{c}}$, Haiying Liu ${ }^{\mathrm{c}}$, Yang Liu ${ }^{\mathrm{d}}$, Jinghong Li ${ }^{\mathrm{d}}$, Xiangqun Zeng ${ }^{\text {a* }}$

${ }^{a}$ Chemistry Department, Oakland University, Rochester 48309, United States

${ }^{b}$ Department of Food Quality and Safety, Jilin University, Changchun 130062, P. R. China

${ }^{c}$ Department of Chemistry, Michigan Technological University, Houghton, 49931, United States

${ }^{\mathrm{d}}$ Department of Chemistry, Tsinghua University, Beijing, 100084, P.R. China

\section{Table of Contents}

Fig.D.1. Electrochemical polymerization of mannosylated aniline monomer on the ITO electrode 202

Fig. D.2. Cyclic voltammograms of manno-PANI film in the absence and presence of different control lectins 203

Fig. D.3. Stability of mannosylated-polyaniline film for sensitive detection of $6 \mathrm{nM}$ Con

A. 203

Figure D.4. ${ }^{1}$ H NMR spectrum of compound 3 ....................................................... 205

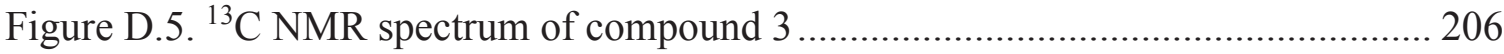

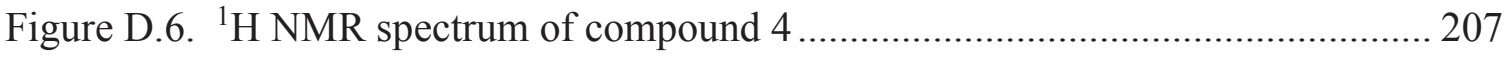

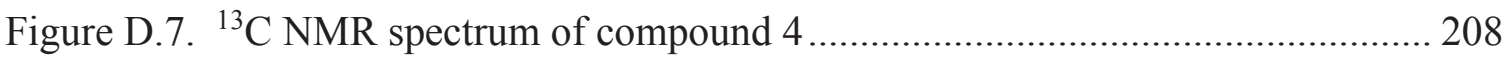

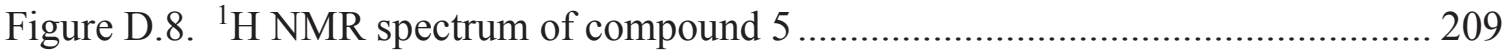

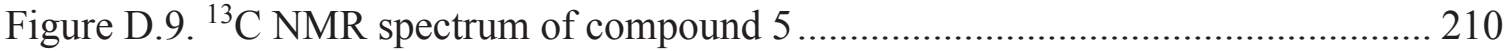

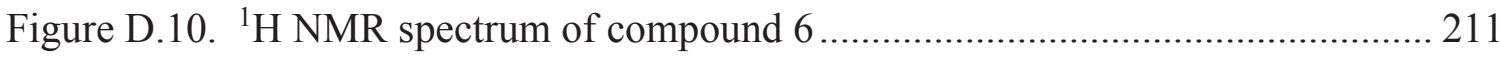

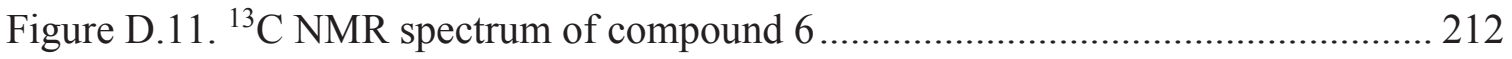




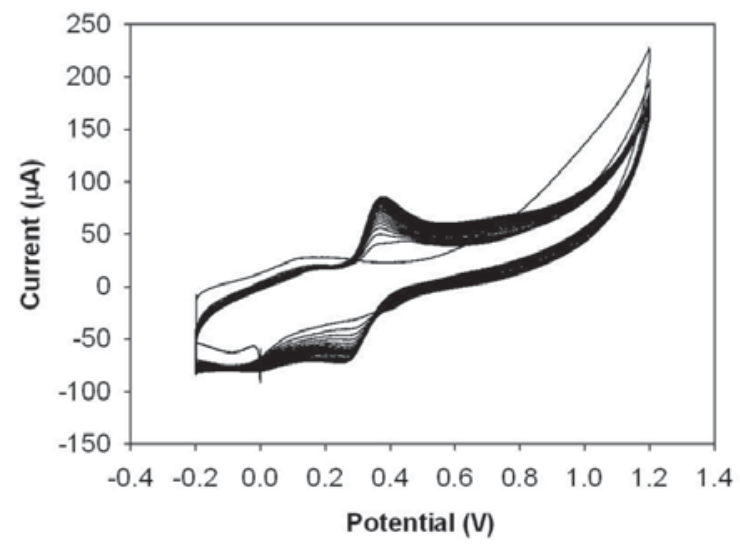

Fig.D.1. Electrochemical polymerization of mannosylated aniline monomer on the ITO electrode in $5 \mathrm{~nm}$ HEPES solution ( $\mathrm{pH}=7.4)$ containing $20 \mathrm{mM}$ mannosylated aniline at a scan rate of $50 \mathrm{mV} / \mathrm{s}$ with 50 scan cycles.
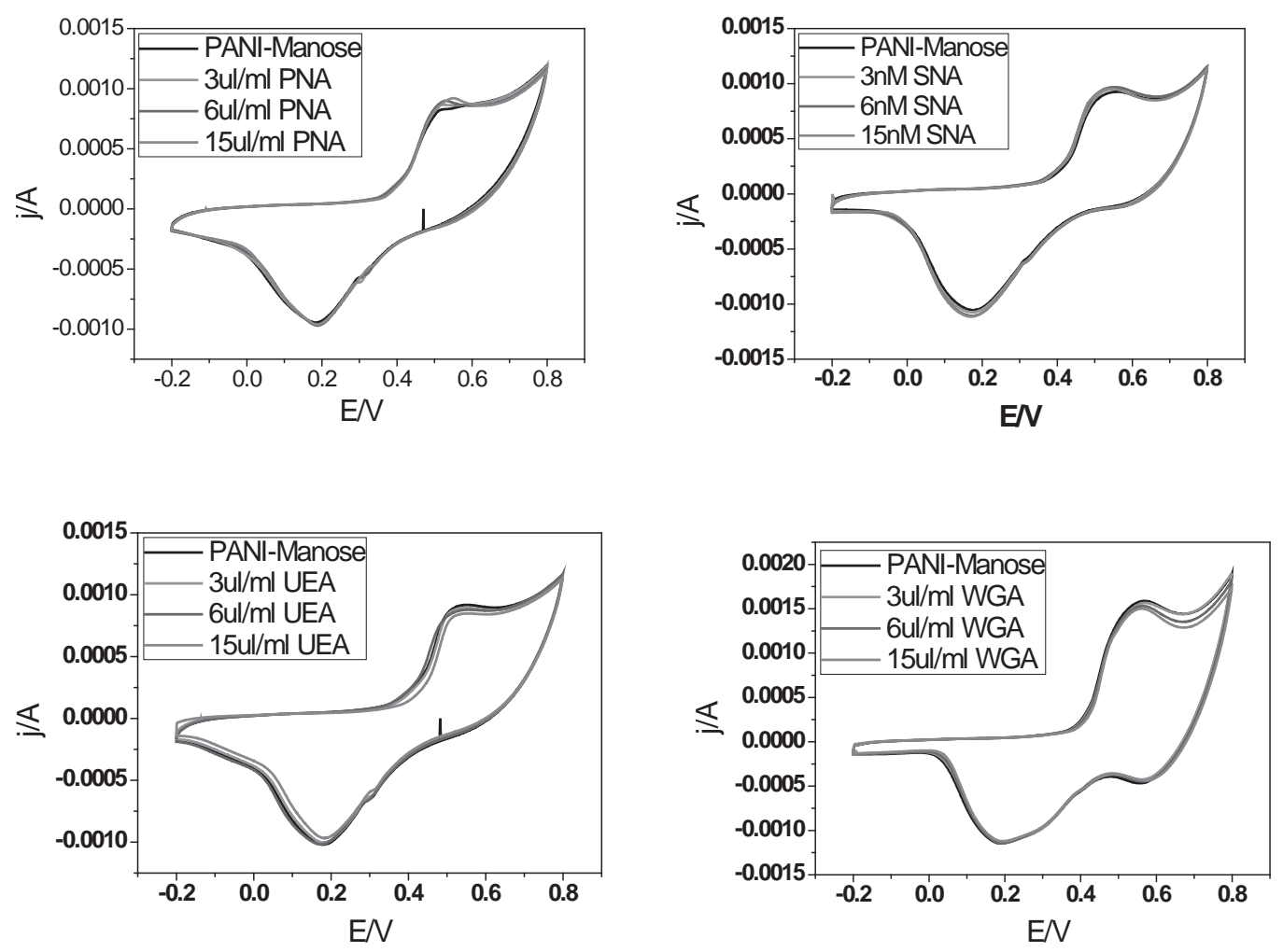
Fig. D.2. Cyclic voltammograms of manno-PANI film in the absence and presence of different control lectins at various concentrations in HEPES buffer with $2 \mathrm{mM} \mathrm{Ca}^{2+}$. Scan rate was $50 \mathrm{mV} / \mathrm{s}, \mathrm{Ag} / \mathrm{AgCl}$ was used as a reference electrode, and Pt wire was used as a counter electrode.

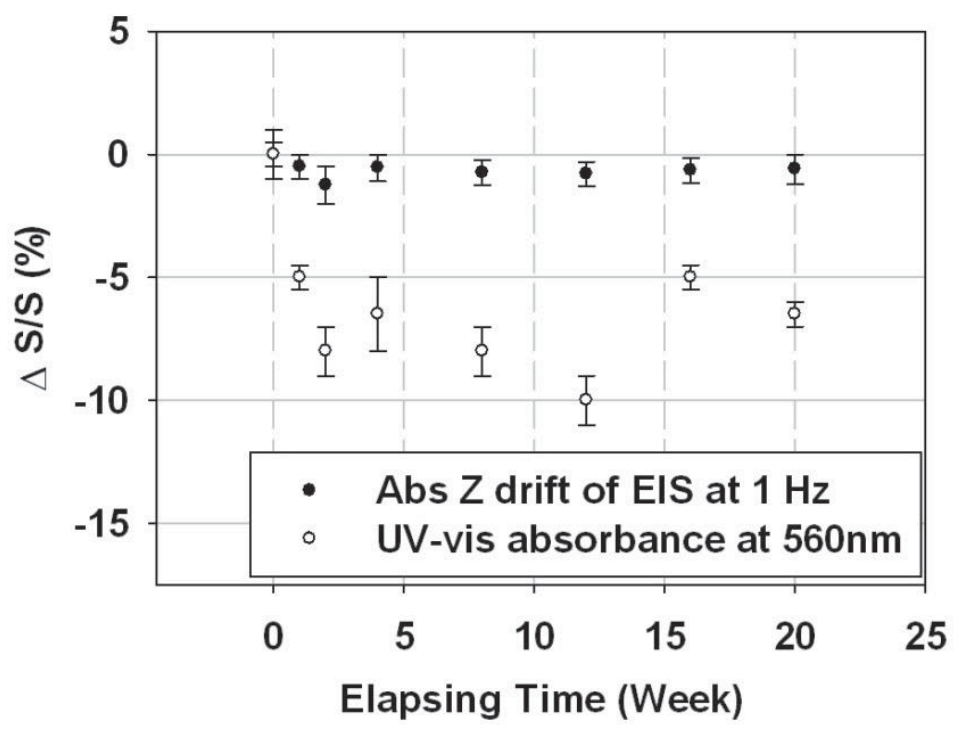

Fig. D.3. Stability of mannosylated-polyaniline film for sensitive detection of $6 \mathrm{nM}$ Con $\mathrm{A}$ in HEPES. $\triangle \mathrm{S} / \mathrm{S}=(\mathrm{S}-\mathrm{S}$ first day $) / \mathrm{S}$ first day. Related data are summarized in Table $\mathrm{S} 1$. 
Table D.1. Stability of mannosylated-polyaniline film for sensitive detection of $6 \mathrm{nM}$ Con $\mathrm{A}$ in HEPES. $\triangle \mathrm{S} / \mathrm{S}=\left(\mathrm{S}-\mathrm{S}_{\text {first day }}\right) / \mathrm{S}$ first day. EIS data were collected under $1 \mathrm{~Hz}$ and UV-vis were collected on $560 \mathrm{~nm}$.

\begin{tabular}{|c|c|c|c|c|c|c|c|c|}
\hline Time & 0 & 1 & 2 & 4 & 8 & 12 & 16 & 20 \\
\hline EIS & 0 & -0.5 & -1.25 & -0.55 & -0.75 & -0.80 & -0.65 & -0.60 \\
& $( \pm 0.51)$ & $( \pm 0.72)$ & $( \pm 0.61)$ & $( \pm 0.52)$ & $( \pm 0.61)$ & $( \pm 0.63)$ & $( \pm 0.57)$ & $( \pm 0.62)$ \\
\hline UV-vis & 0 & -5 & -8 & -6.5 & -8 & -10 & -5 & -6.5 \\
& $( \pm 1.1)$ & $( \pm 0.6)$ & $( \pm 1.2)$ & $( \pm 1.4)$ & $( \pm 1.2)$ & $( \pm 1.1)$ & $( \pm 0.6)$ & $( \pm 0.5)$ \\
\hline
\end{tabular}




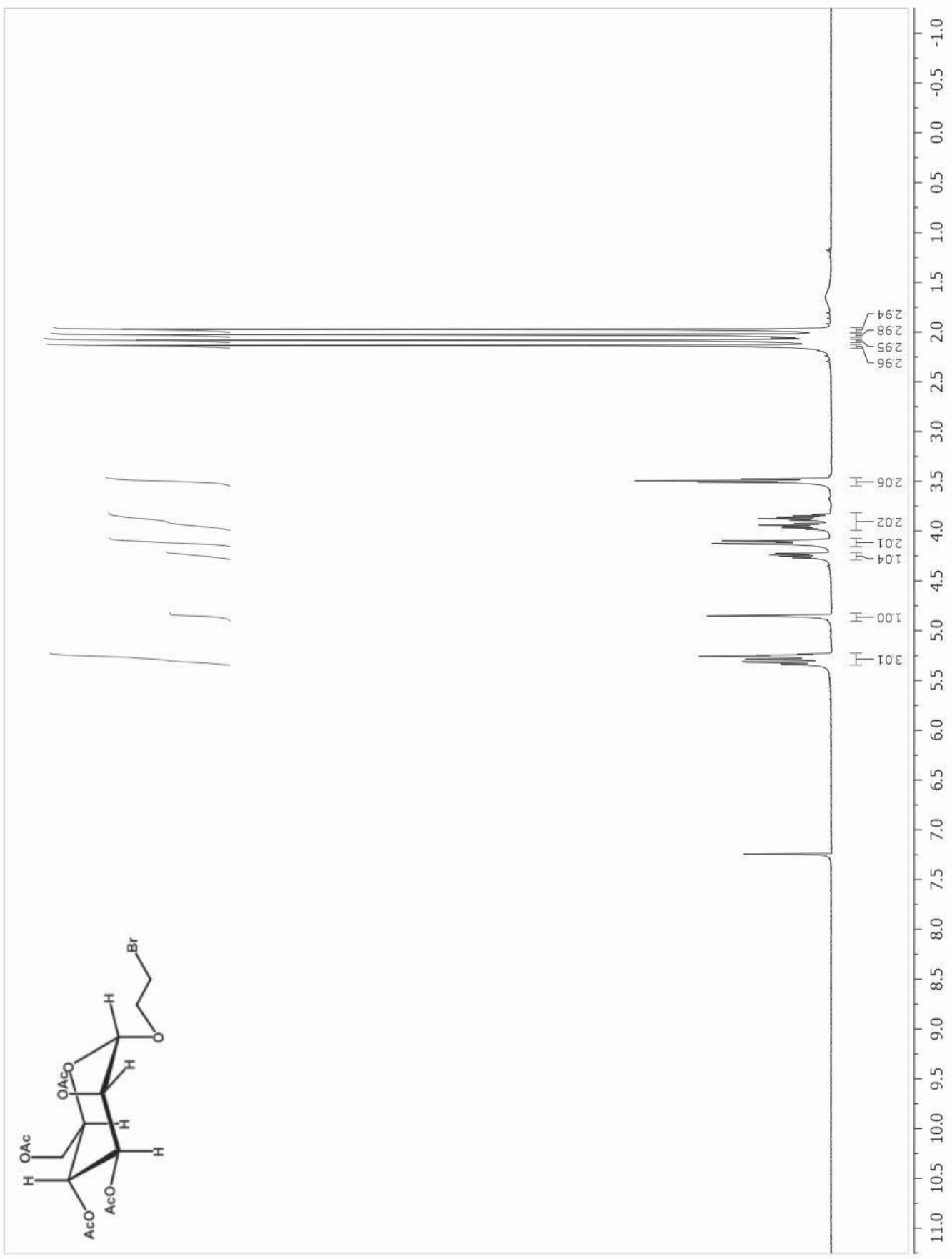

Figure D.4. ${ }^{1} \mathrm{H}$ NMR spectrum of compound 3 


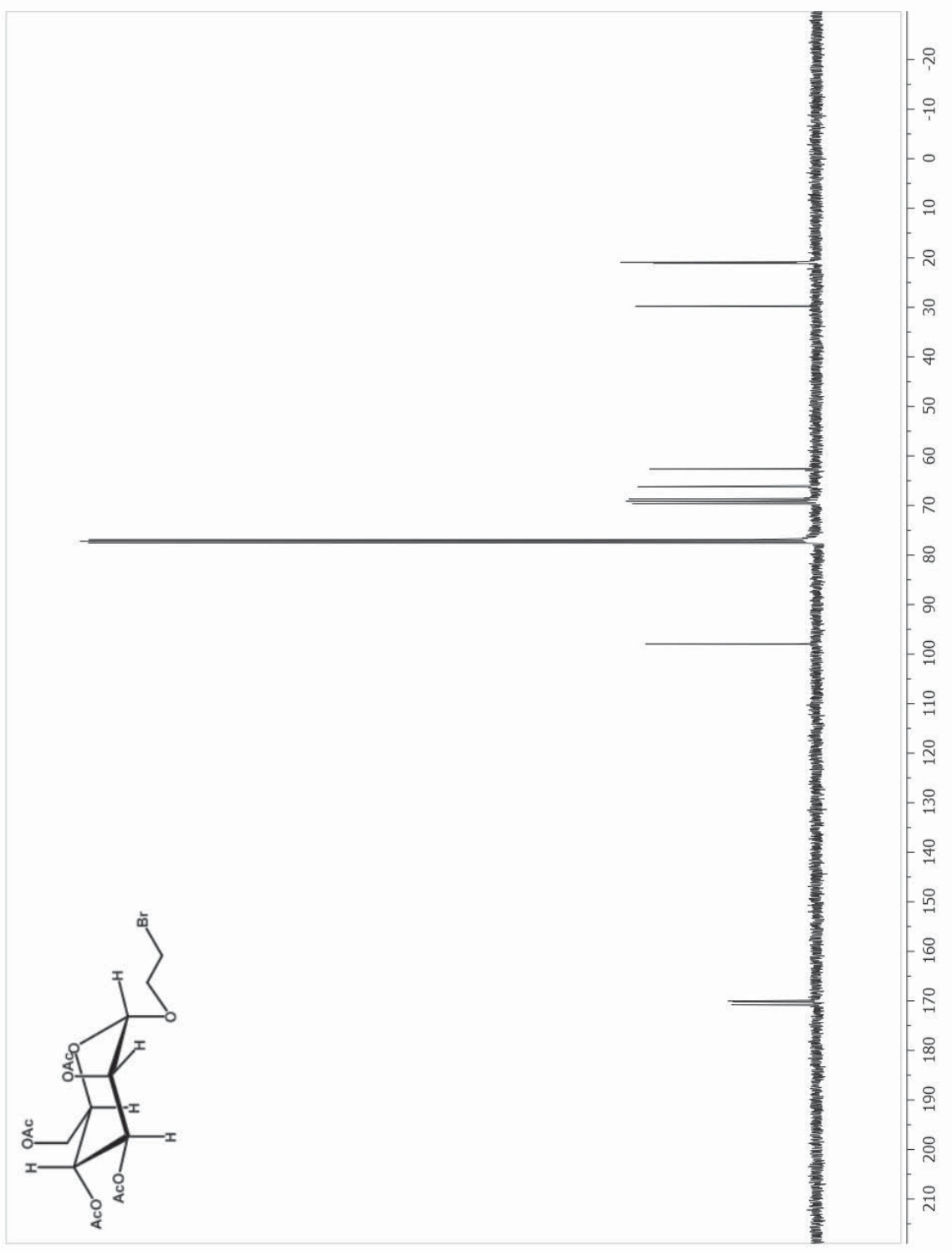

Figure D.5. ${ }^{13} \mathrm{C}$ NMR spectrum of compound $\mathbf{3}$ 


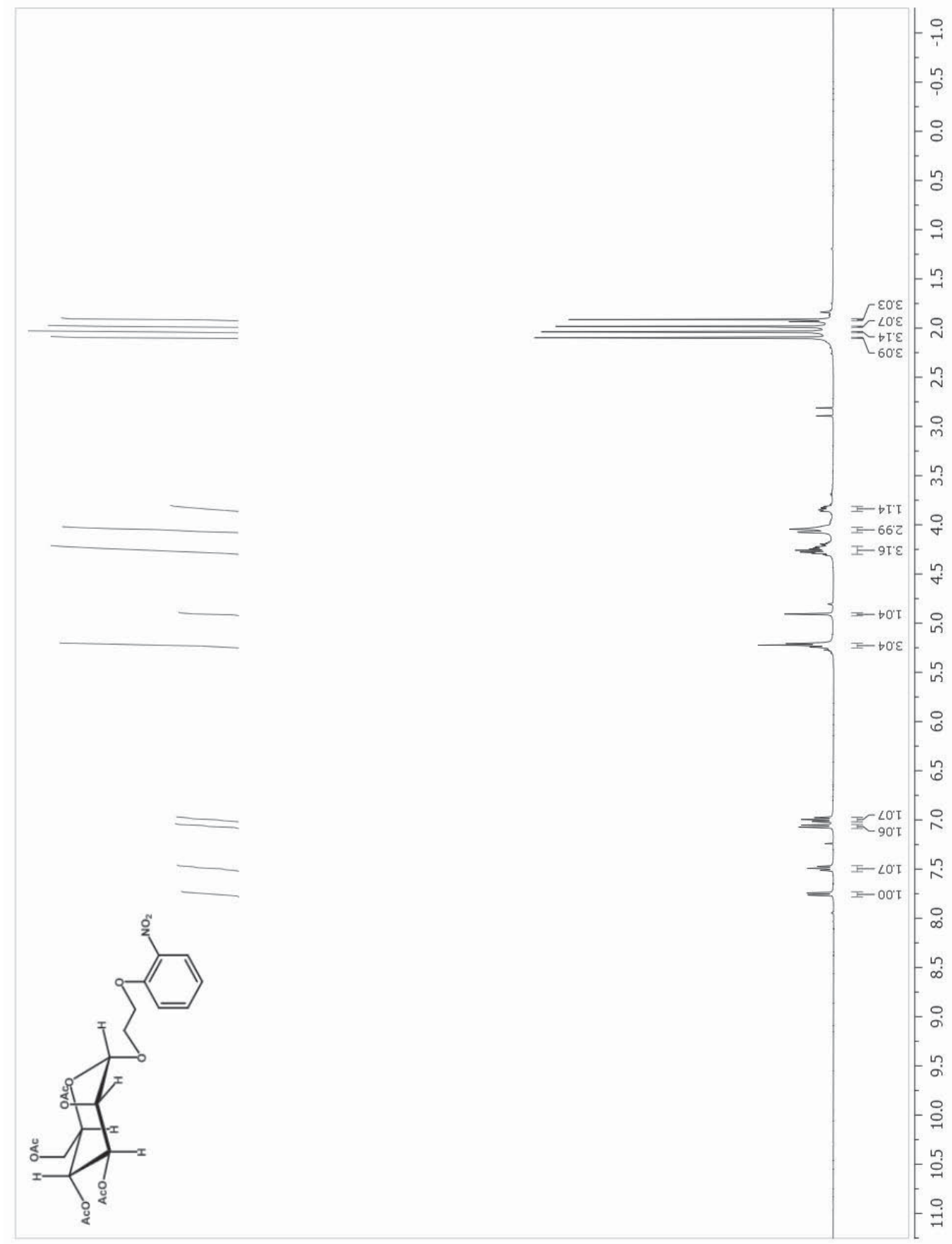

Figure D.6. ${ }^{1} \mathrm{H}$ NMR spectrum of compound 4 


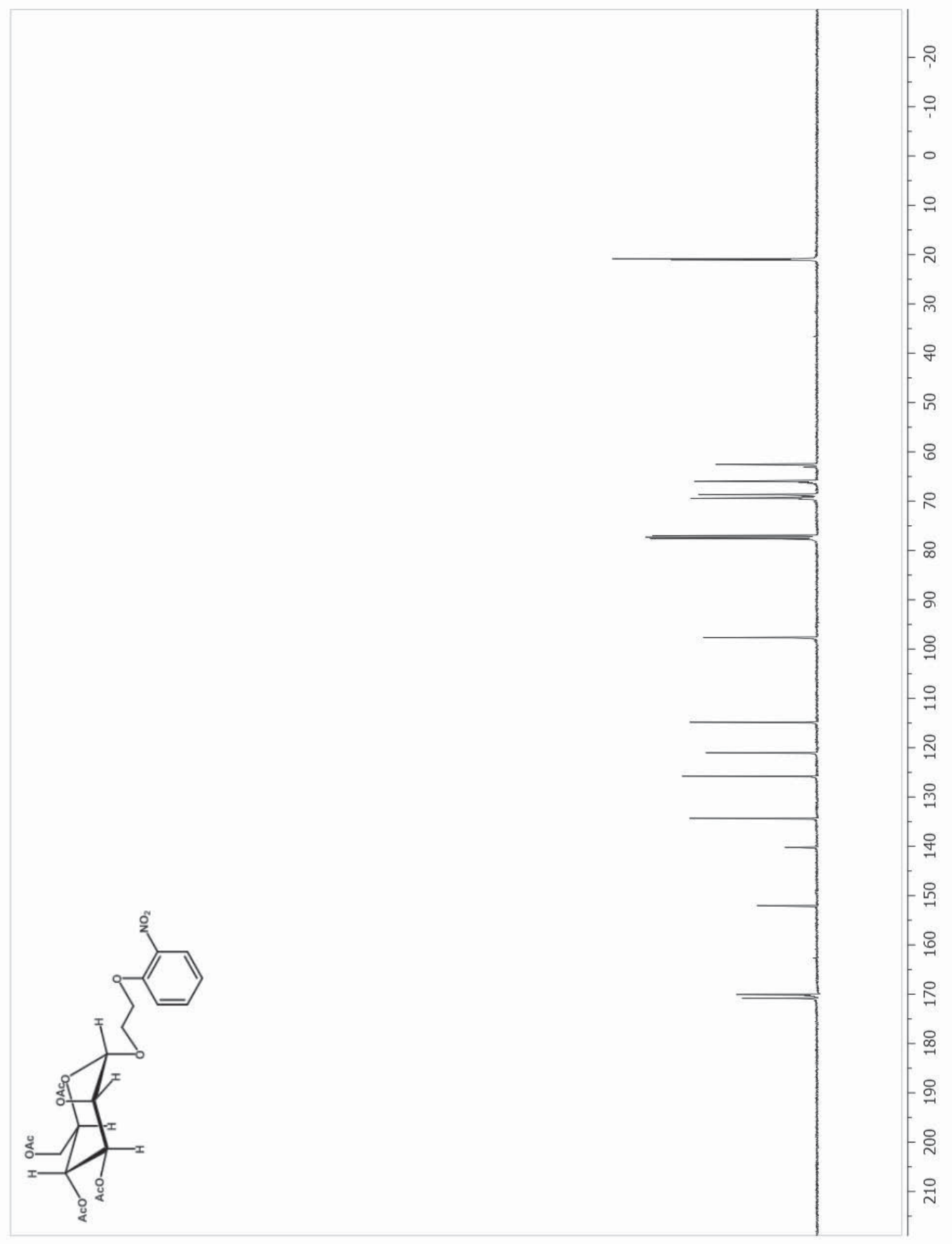

Figure D.7. ${ }^{13} \mathrm{C}$ NMR spectrum of compound 4 


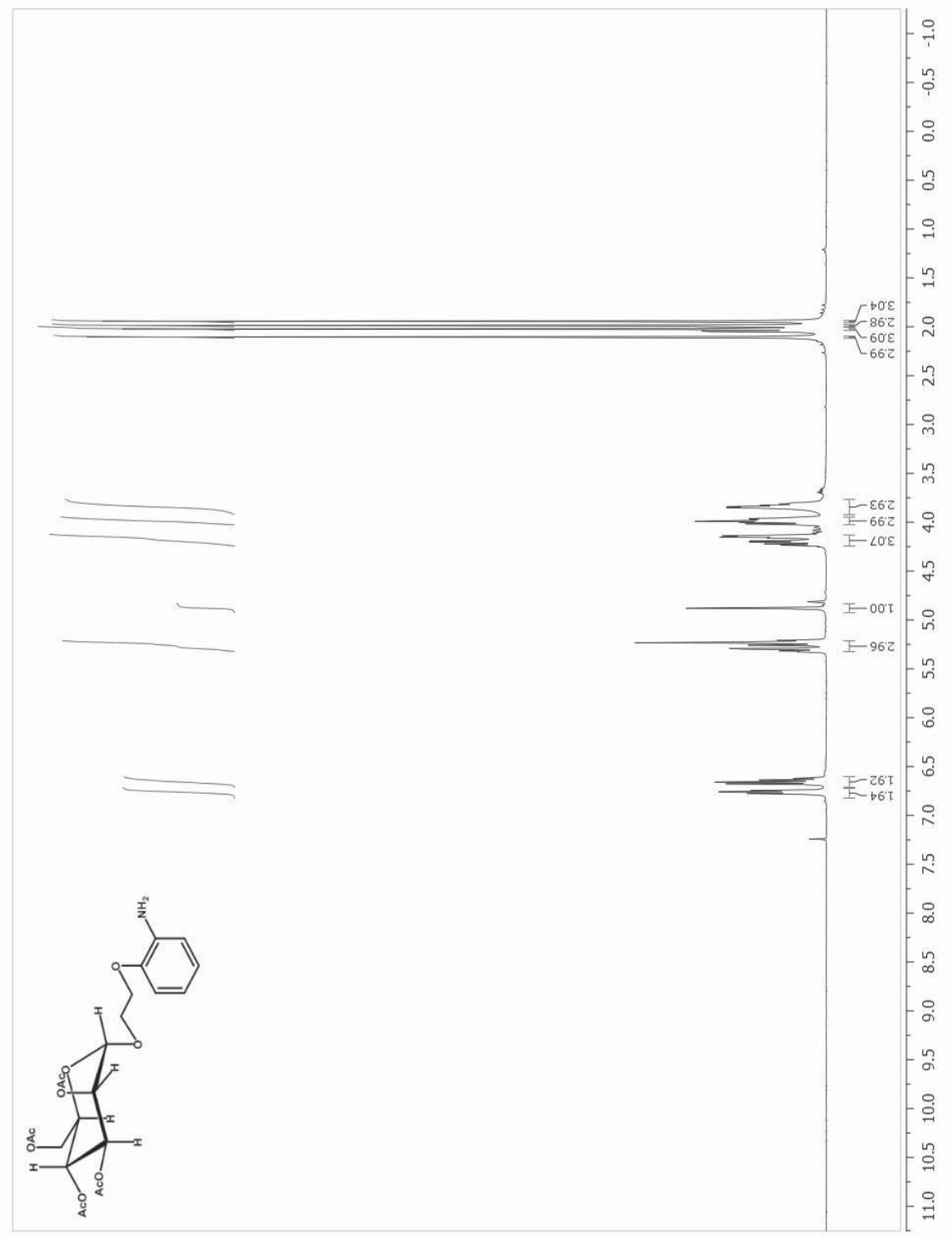

Figure D.8. ${ }^{1} \mathrm{H}$ NMR spectrum of compound 5 


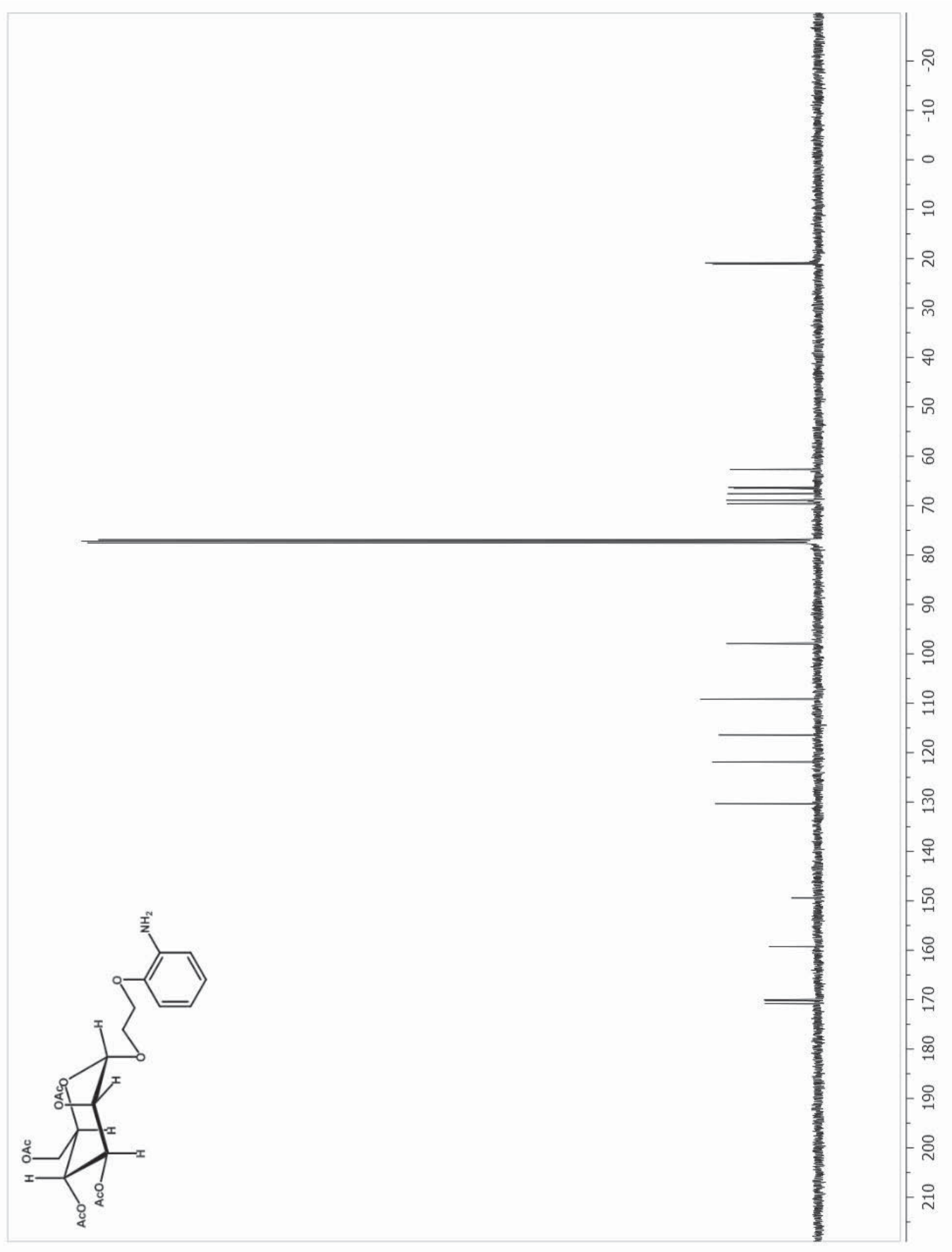

Figure D.9. ${ }^{13} \mathrm{C}$ NMR spectrum of compound 5 


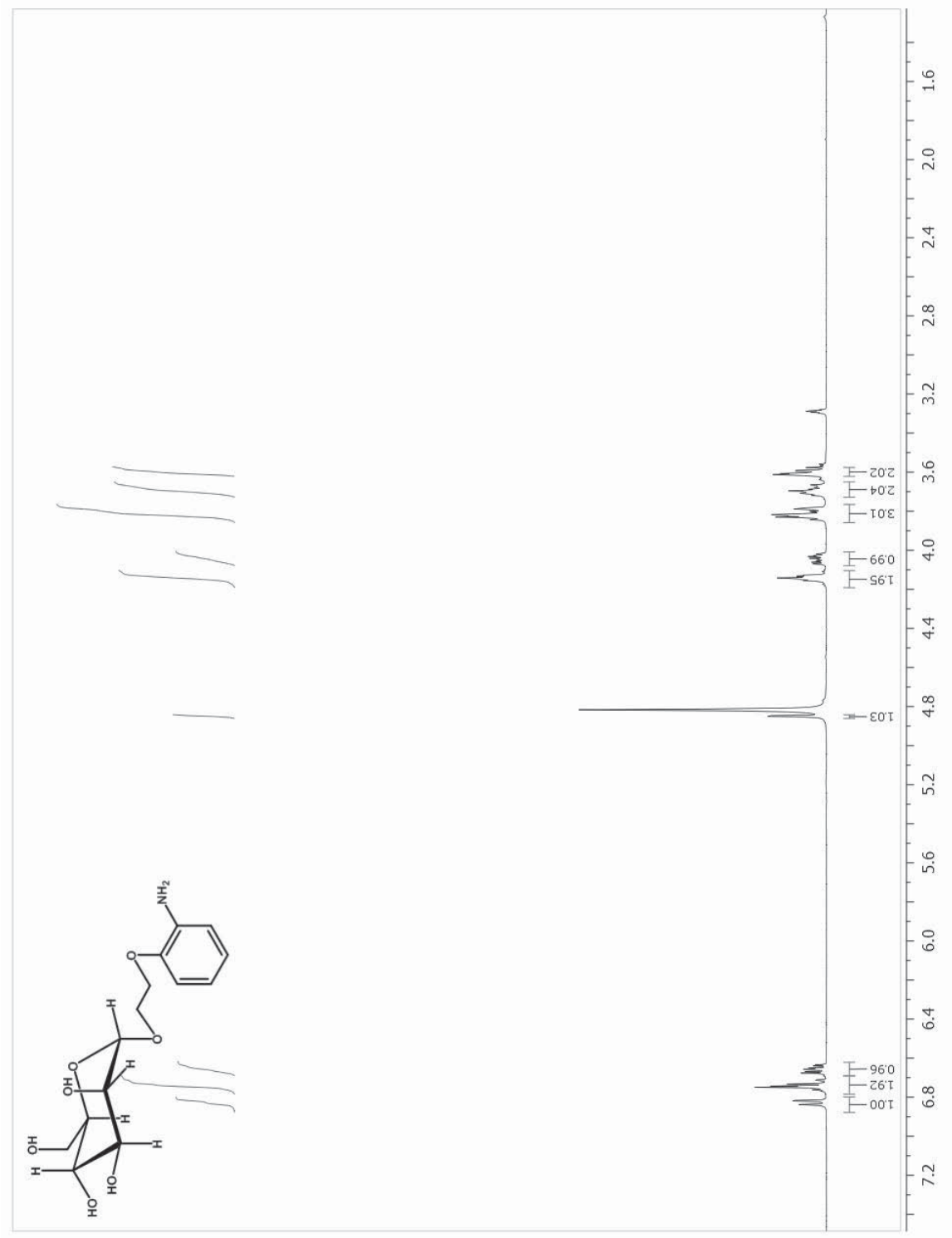

Figure D.10. ${ }^{1} \mathrm{H}$ NMR spectrum of compound 6 


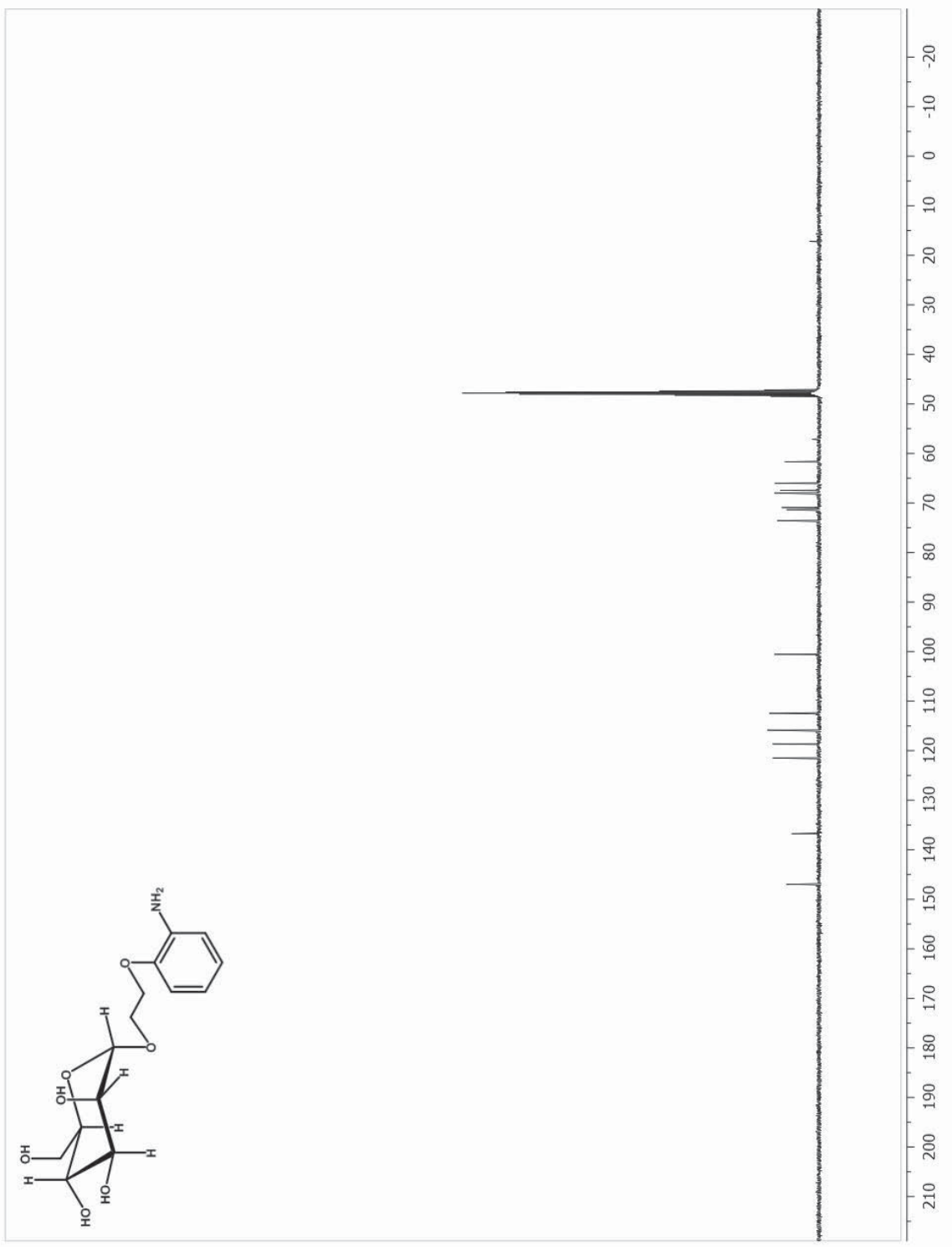

Figure D.11. ${ }^{13} \mathrm{C}$ NMR spectrum of compound 6 


\section{APPENDIX E \\ SUPPORTING INFORMSTION FOR CHAPTER 6 \\ Ferrocene-Mannose Conjugate: A Potential Electrochemical Probe \\ for Carbohydrate-Lectin Interactions}

Giri Vegesna, ${ }^{\mathrm{a}}$ Fen-Tair Luo ${ }^{\mathrm{b}}$, and Haiying Liu ${ }^{\mathrm{a} *}$

a'Department of Chemistry, Michigan Technological University, Houghton, MI 49931

${ }^{b}$ Institute of Chemistry, Academia Sinica, Taipei, Taiwan 11529, Republic of China

\section{Table of Contents}

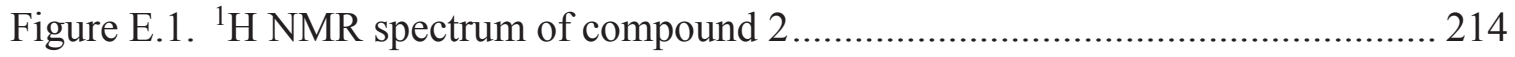

Figure E.2. ${ }^{13} \mathrm{C}$ NMR spectrum of compound 2 ……......................................... 215

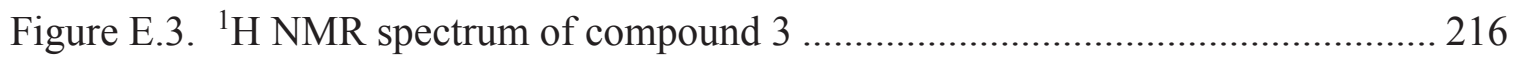

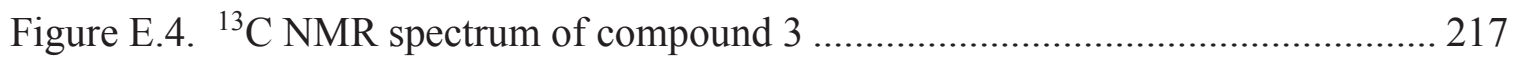

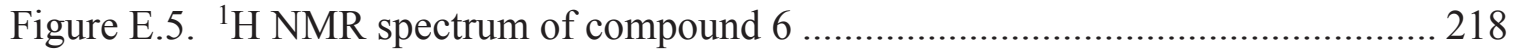

Figure E.6. ${ }^{13} \mathrm{C}$ NMR spectrum of compound 6 .................................................... 219

Figure E.7. ${ }^{1} \mathrm{H}$ NMR spectrum of compound 7 ..................................................... 220

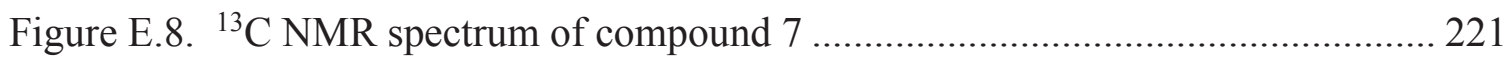

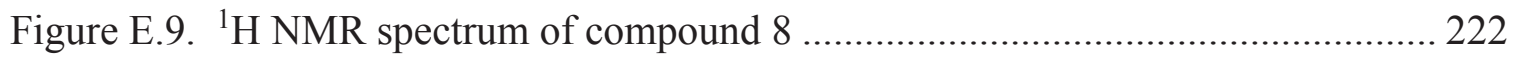

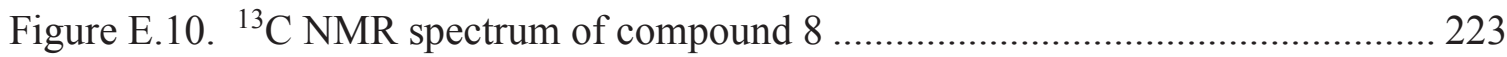

Figure E.11. ${ }^{1} \mathrm{H}$ NMR spectrum of compound 9 ………………………………..... 224

Figure E.12. ${ }^{13} \mathrm{C}$ NMR spectrum of compound 9 ………........................................ 225

Figure E.13. ${ }^{1} \mathrm{H}$ NMR spectrum of compound 10 .................................................... 226

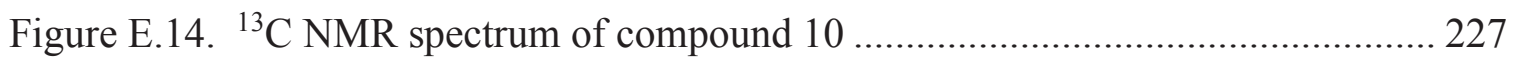




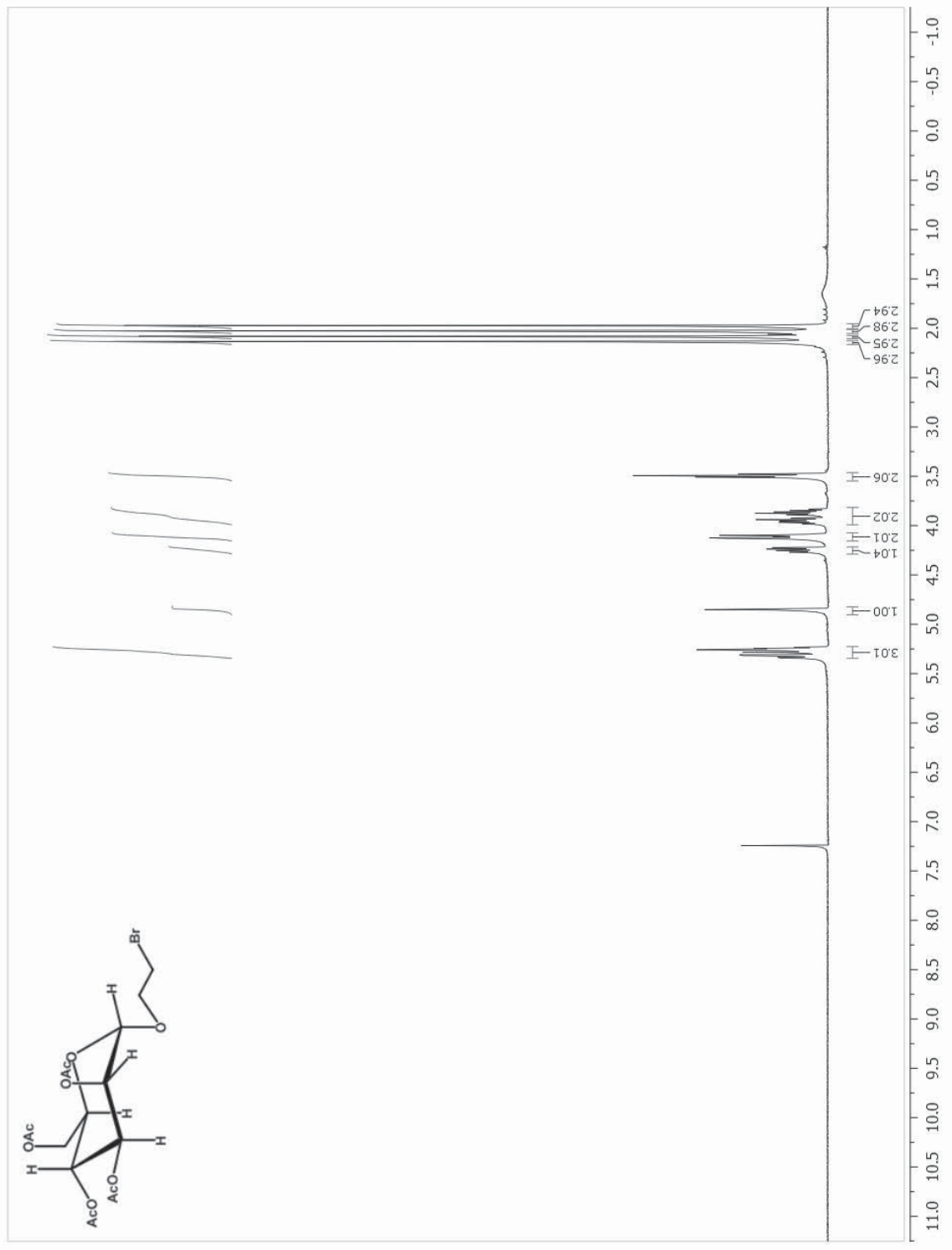

Figure E.1. ${ }^{1} \mathrm{H}$ NMR spectrum of compound 2 


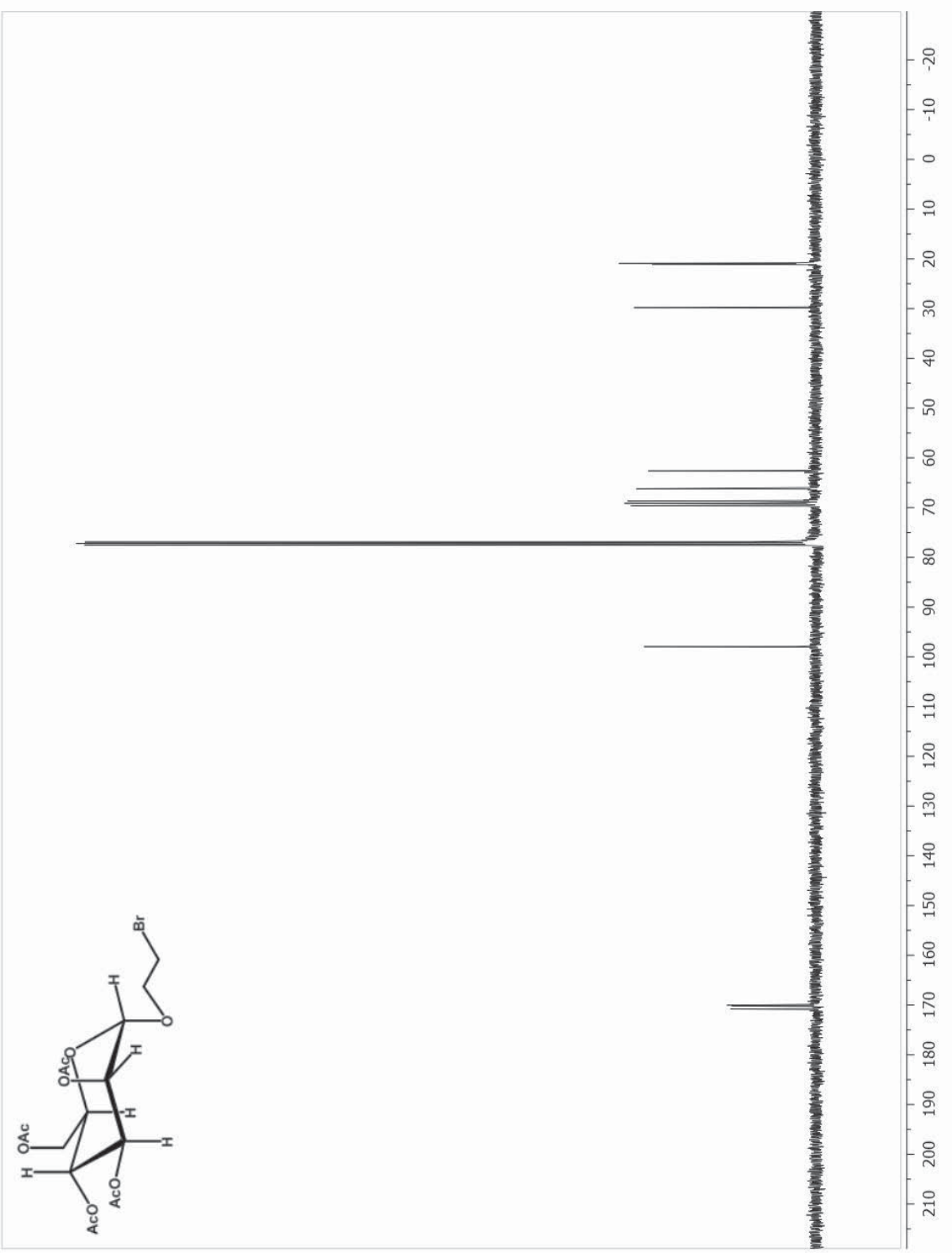

Figure E.2. ${ }^{13} \mathrm{C}$ NMR spectrum of compound 2 


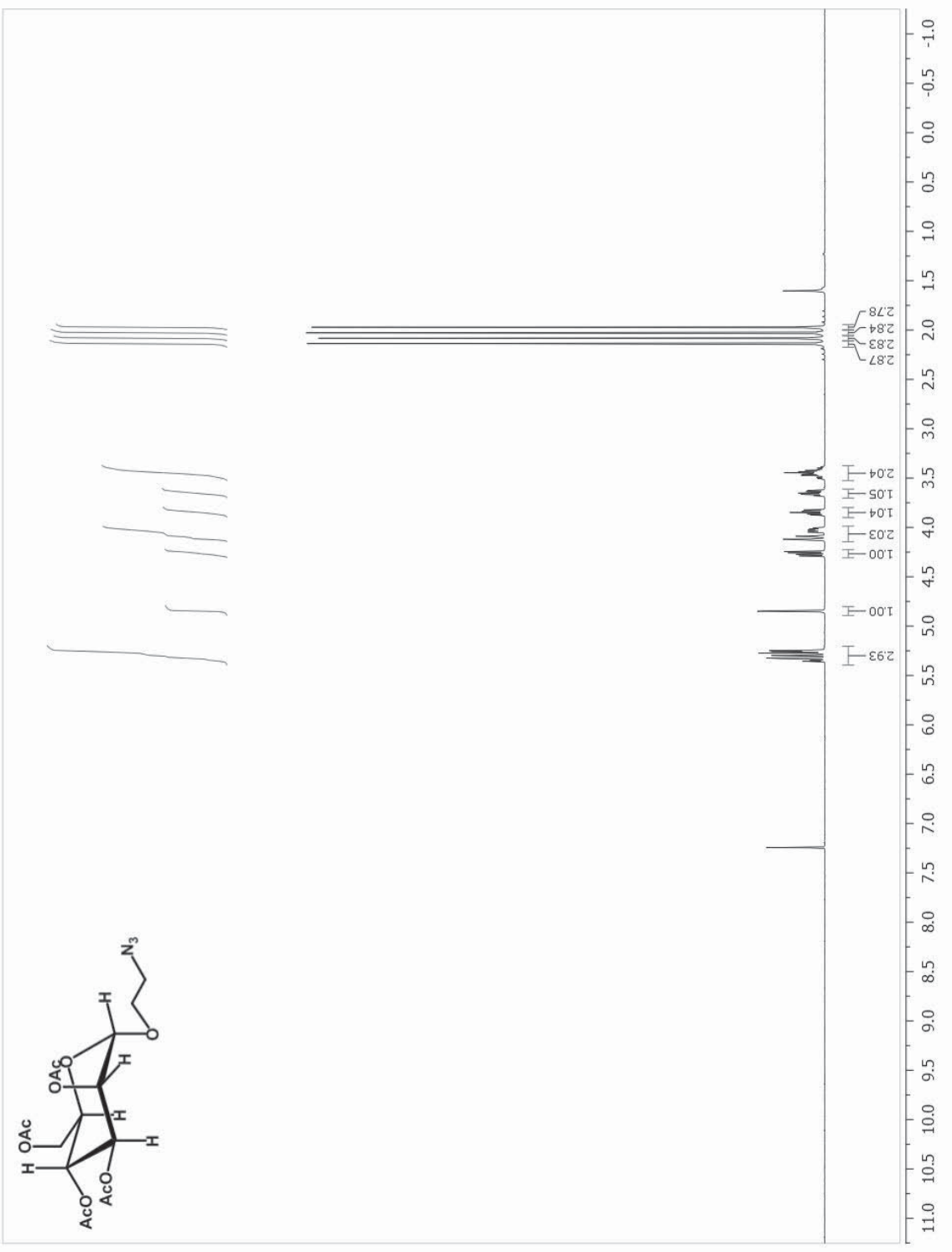

Figure E.3. ${ }^{1} \mathrm{H}$ NMR spectrum of compound $\mathbf{3}$ 


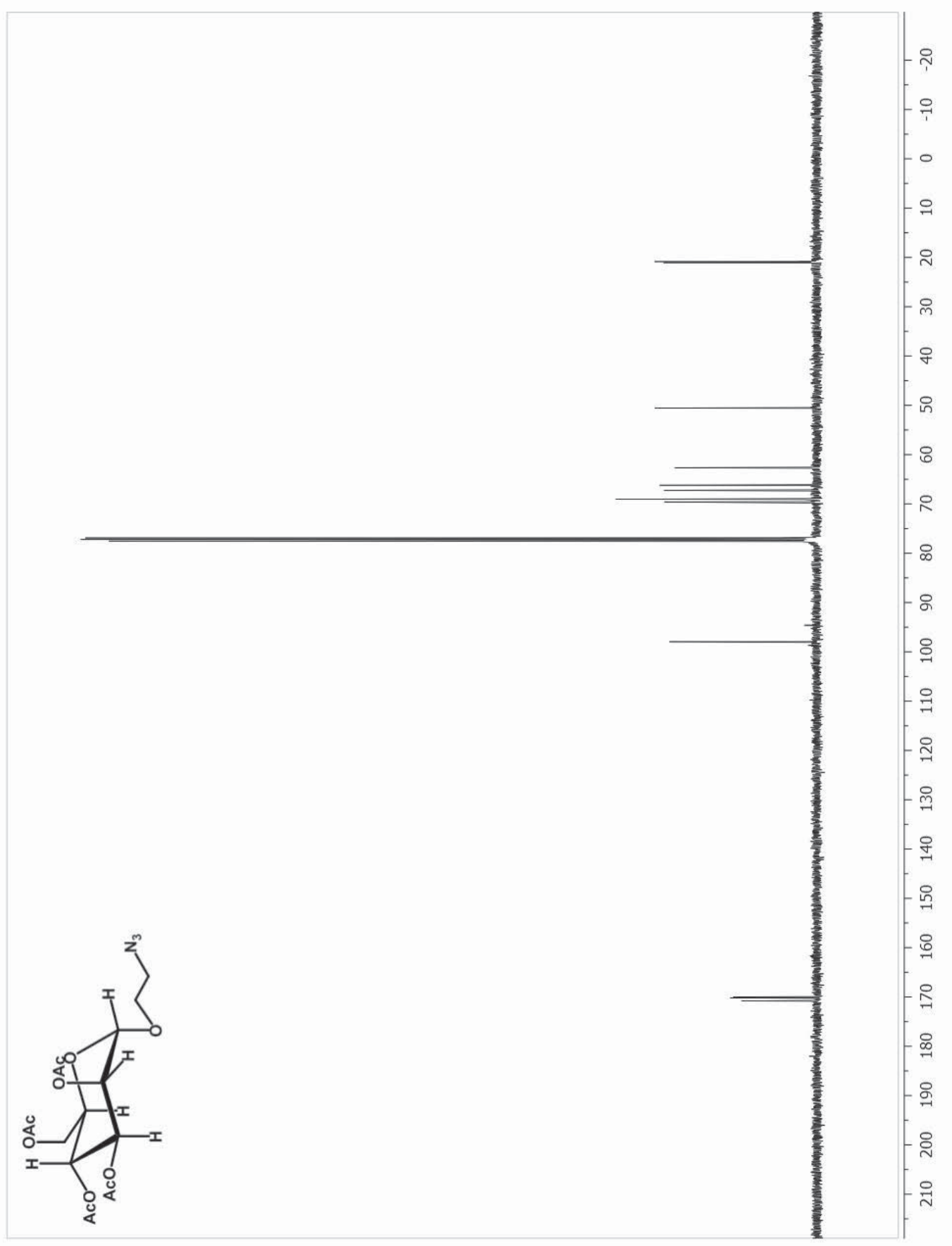

Figure E.4. ${ }^{13} \mathrm{C}$ NMR spectrum of compound $\mathbf{3}$ 


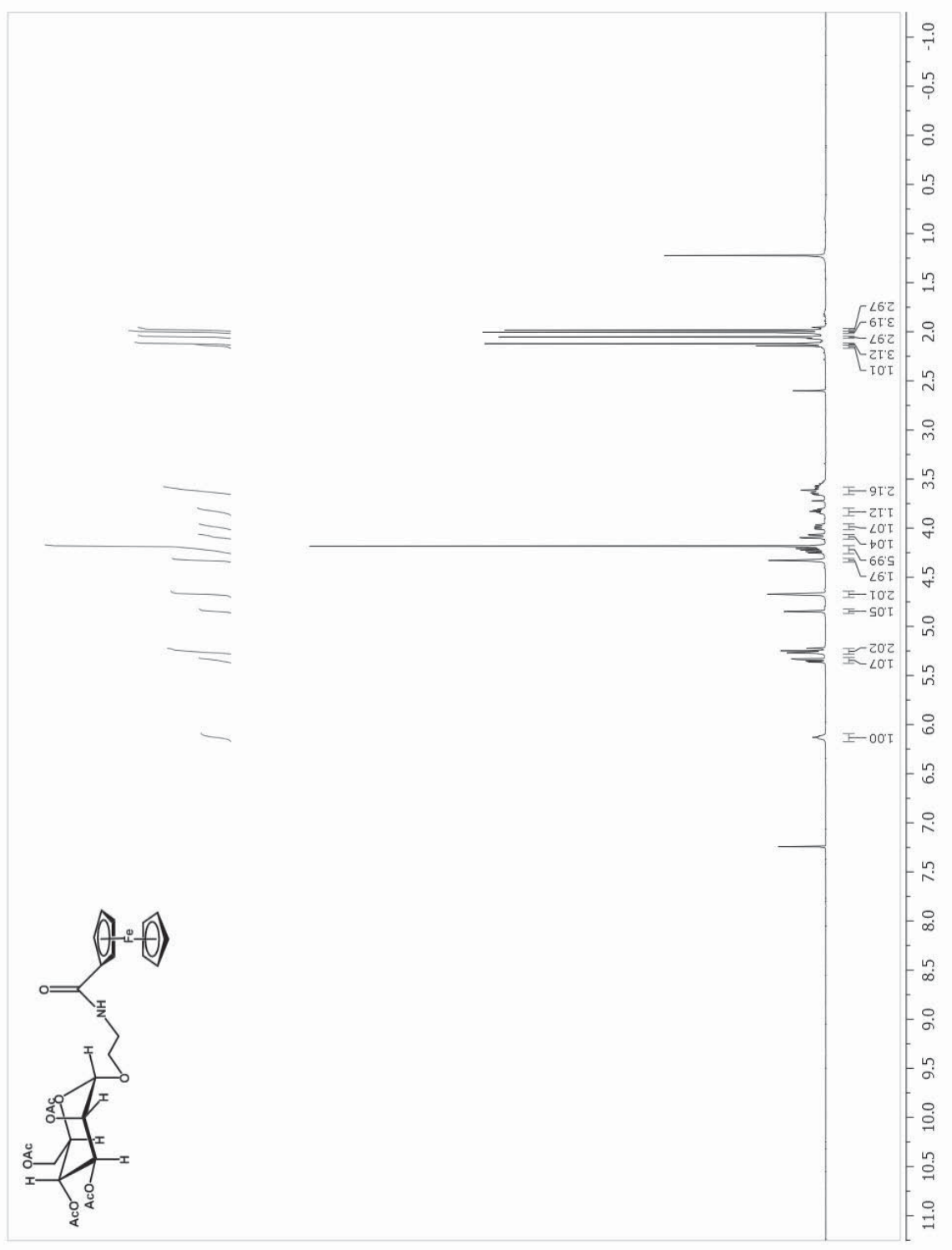

Figure E.5. ${ }^{1} \mathrm{H}$ NMR spectrum of compound 6 


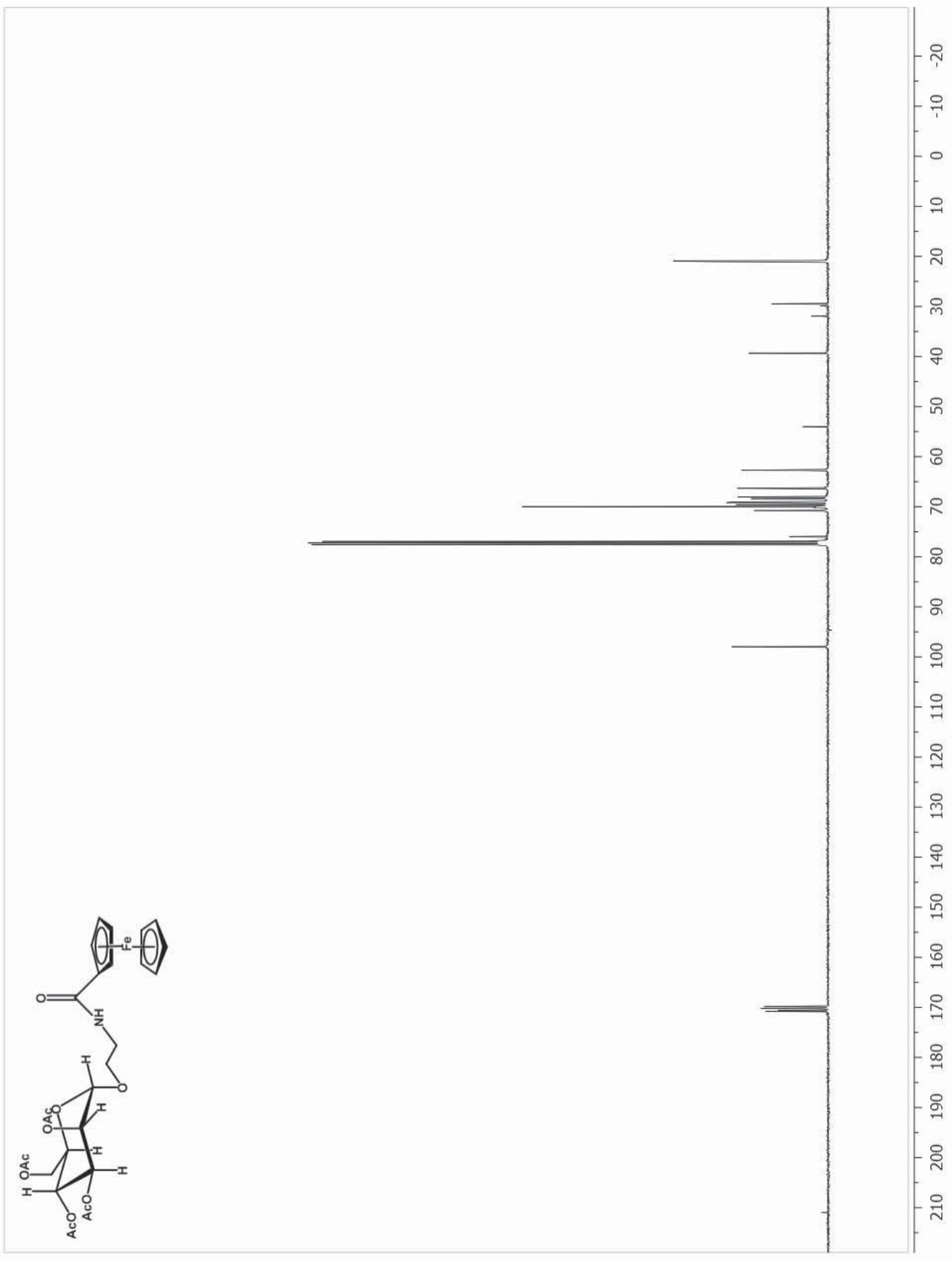

Figure E.6. ${ }^{13} \mathrm{C}$ NMR spectrum of compound 6 


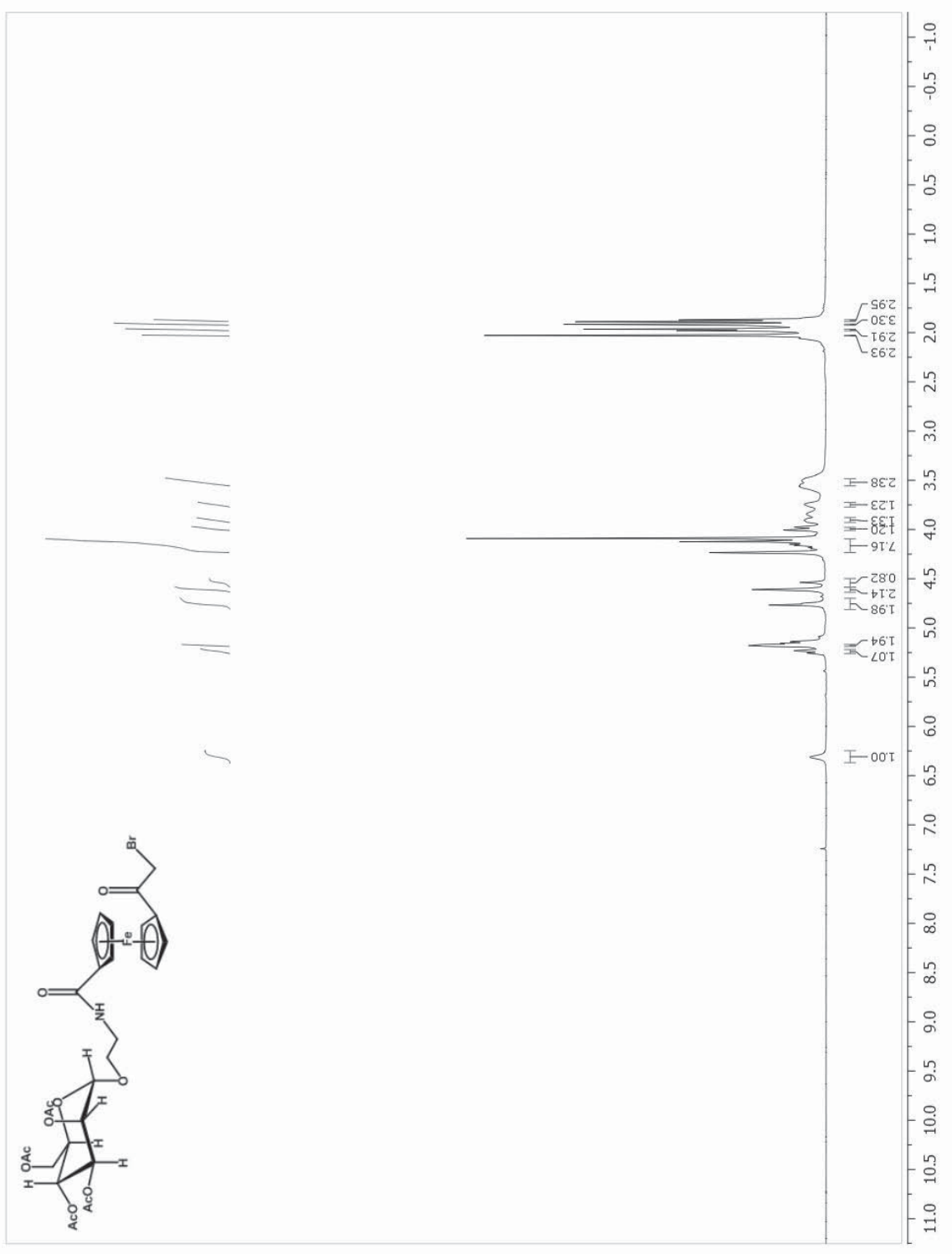

Figure E.7. ${ }^{1} \mathrm{H}$ NMR spectrum of compound 7 


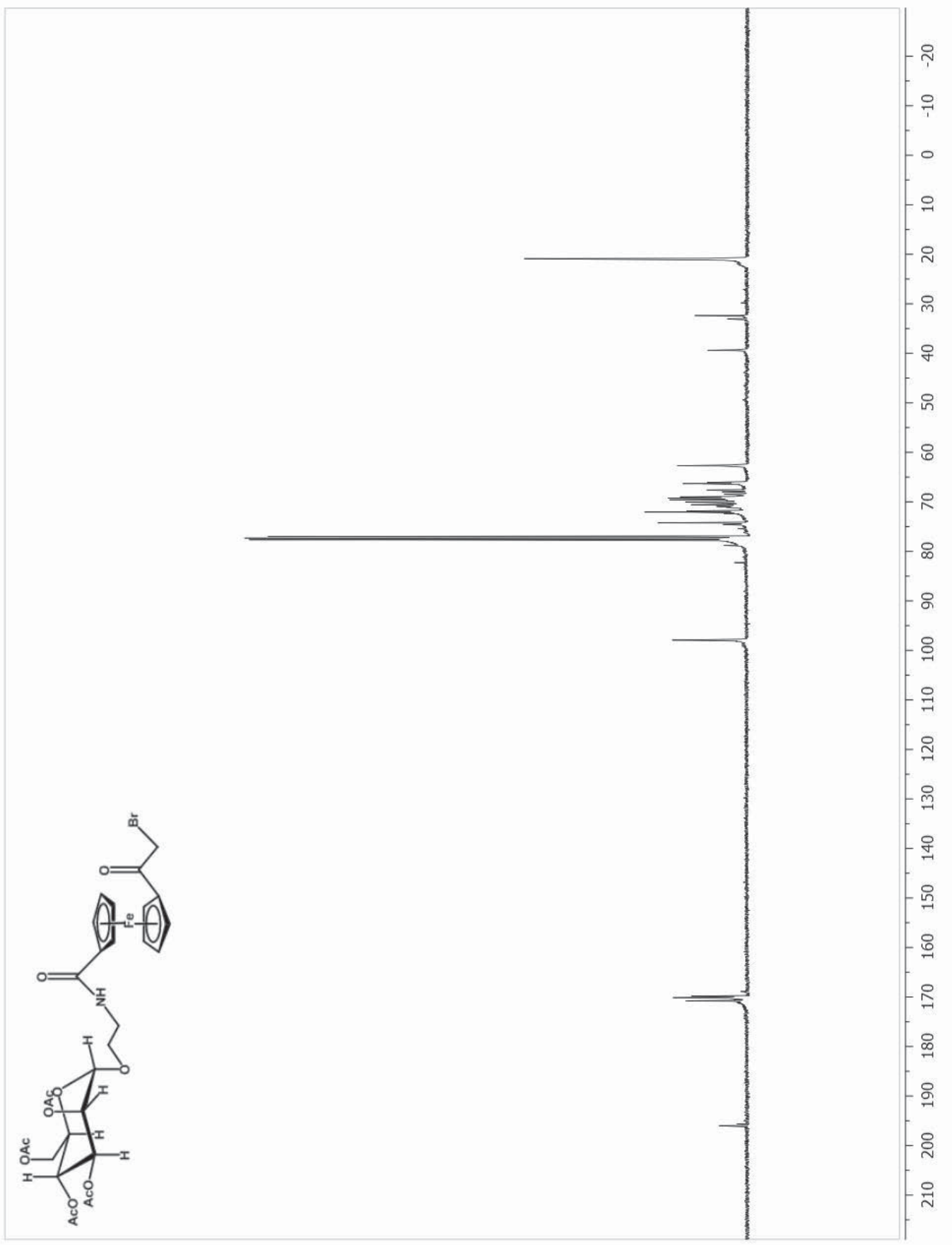

Figure E.8. ${ }^{13} \mathrm{C}$ NMR spectrum of compound 7 


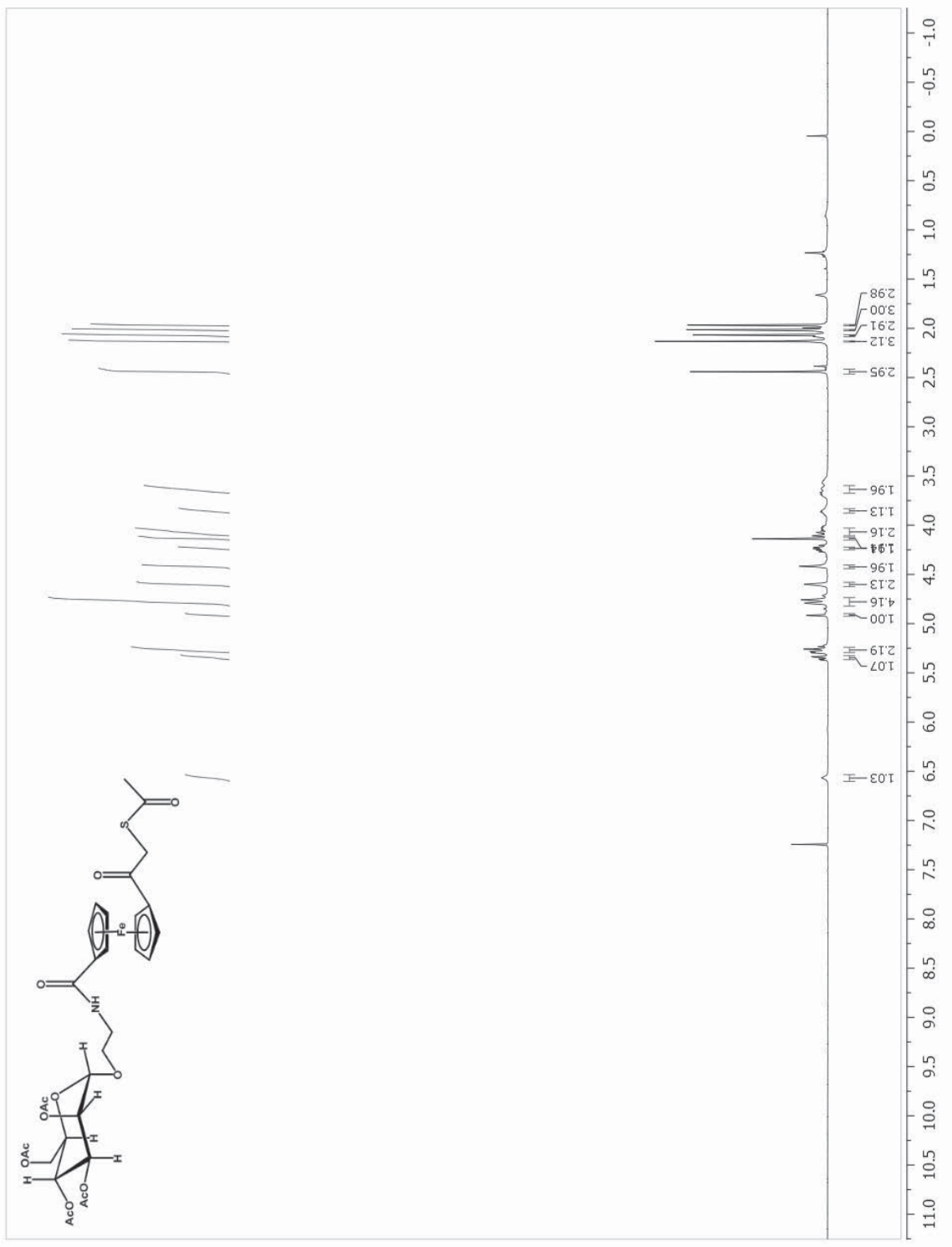

Figure E.9. ${ }^{1} \mathrm{H}$ NMR spectrum of compound 8 


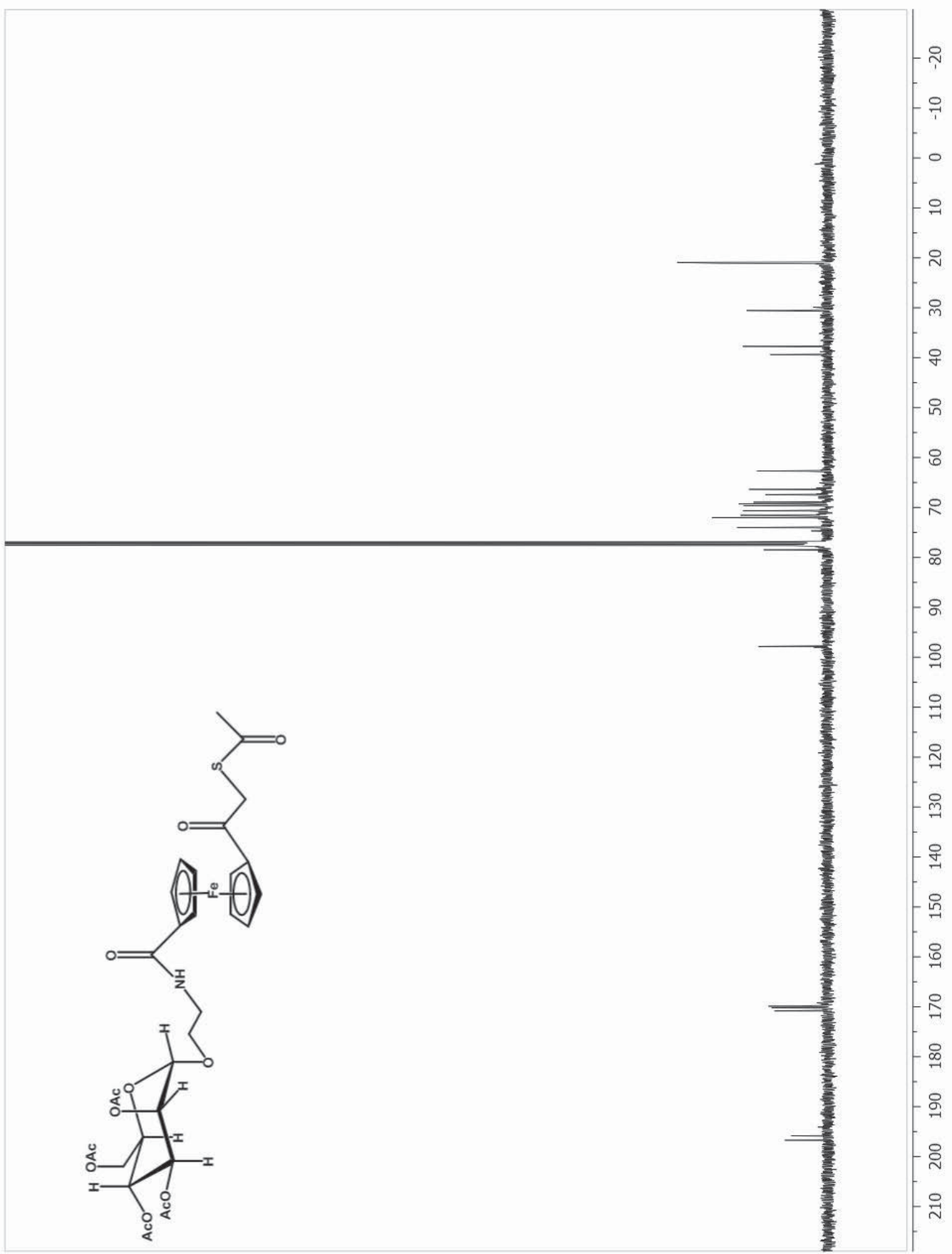

Figure E.10. ${ }^{13} \mathrm{C}$ NMR spectrum of compound 8 


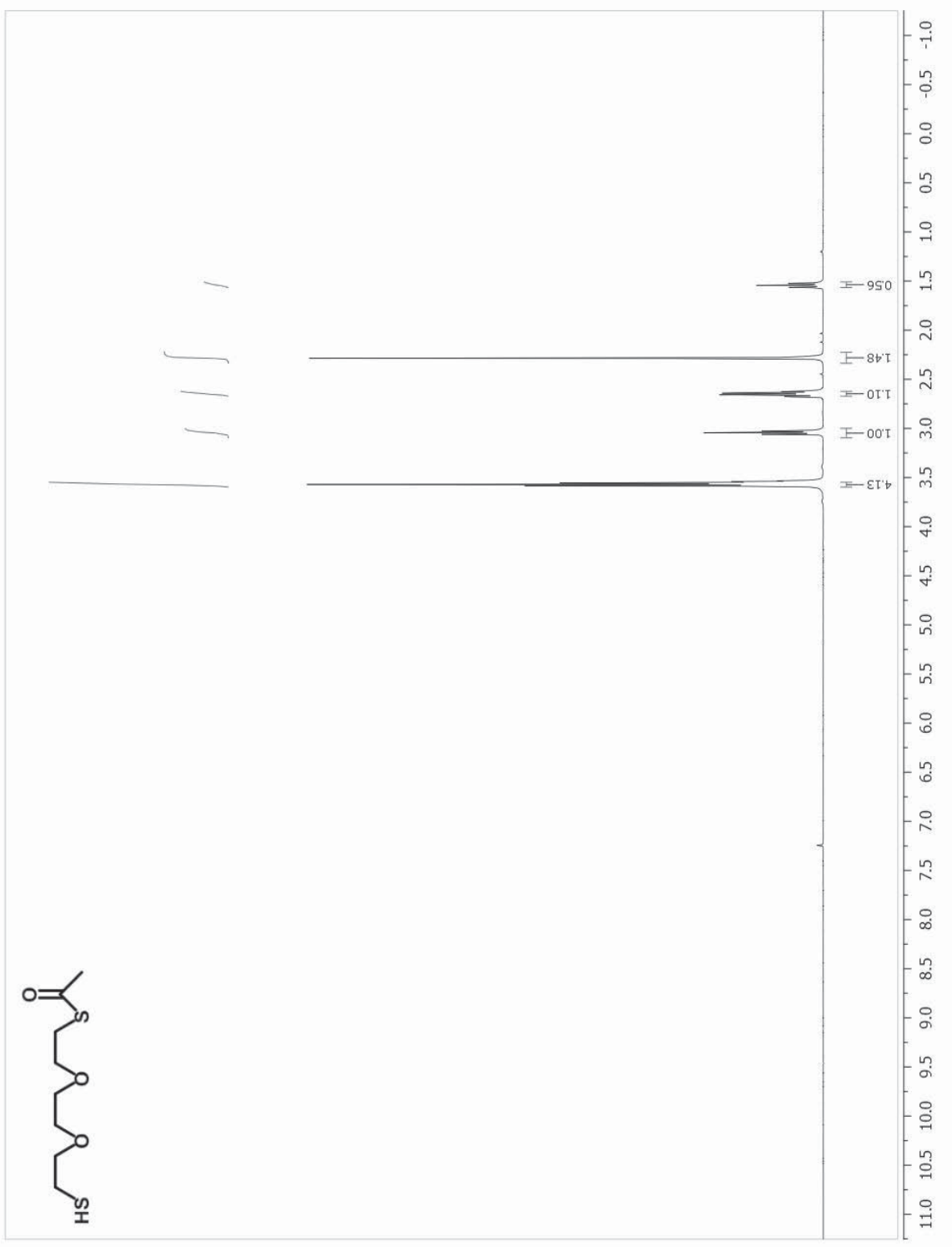

Figure E.11. ${ }^{1} \mathrm{H}$ NMR spectrum of compound 9 


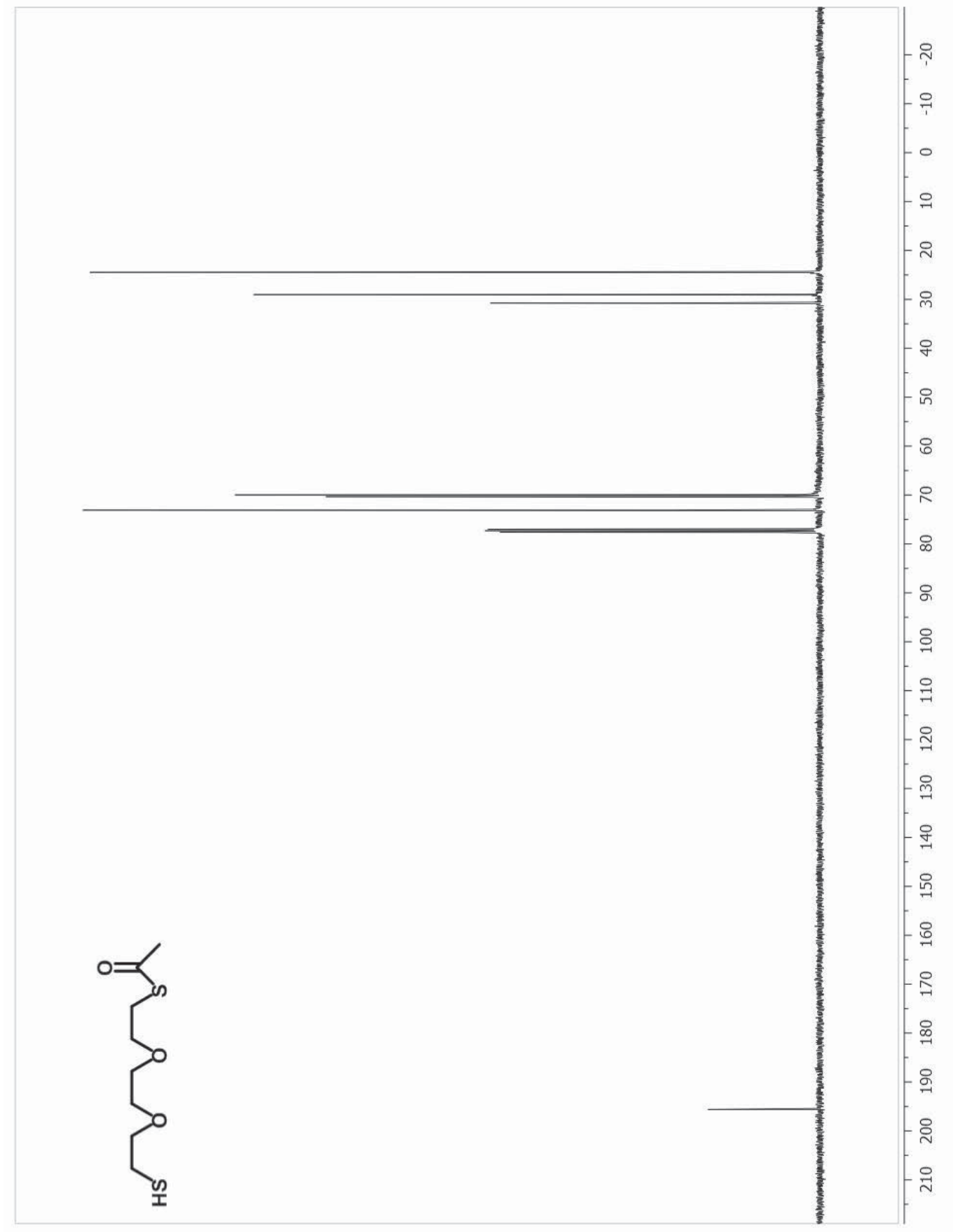

Figure E.12. ${ }^{13} \mathrm{C}$ NMR spectrum of compound 9 


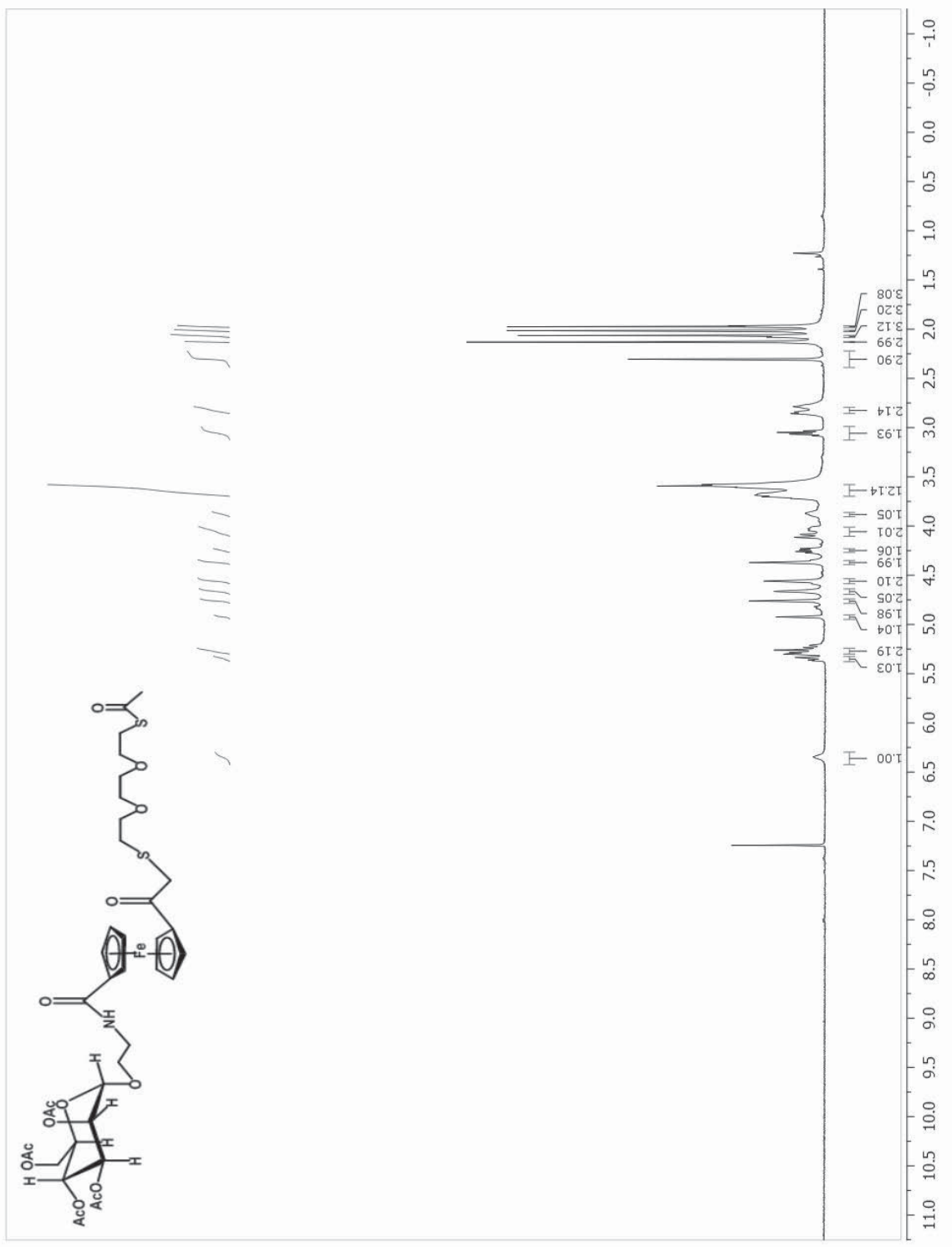

Figure E.13. ${ }^{1} \mathrm{H}$ NMR spectrum of compound 10 


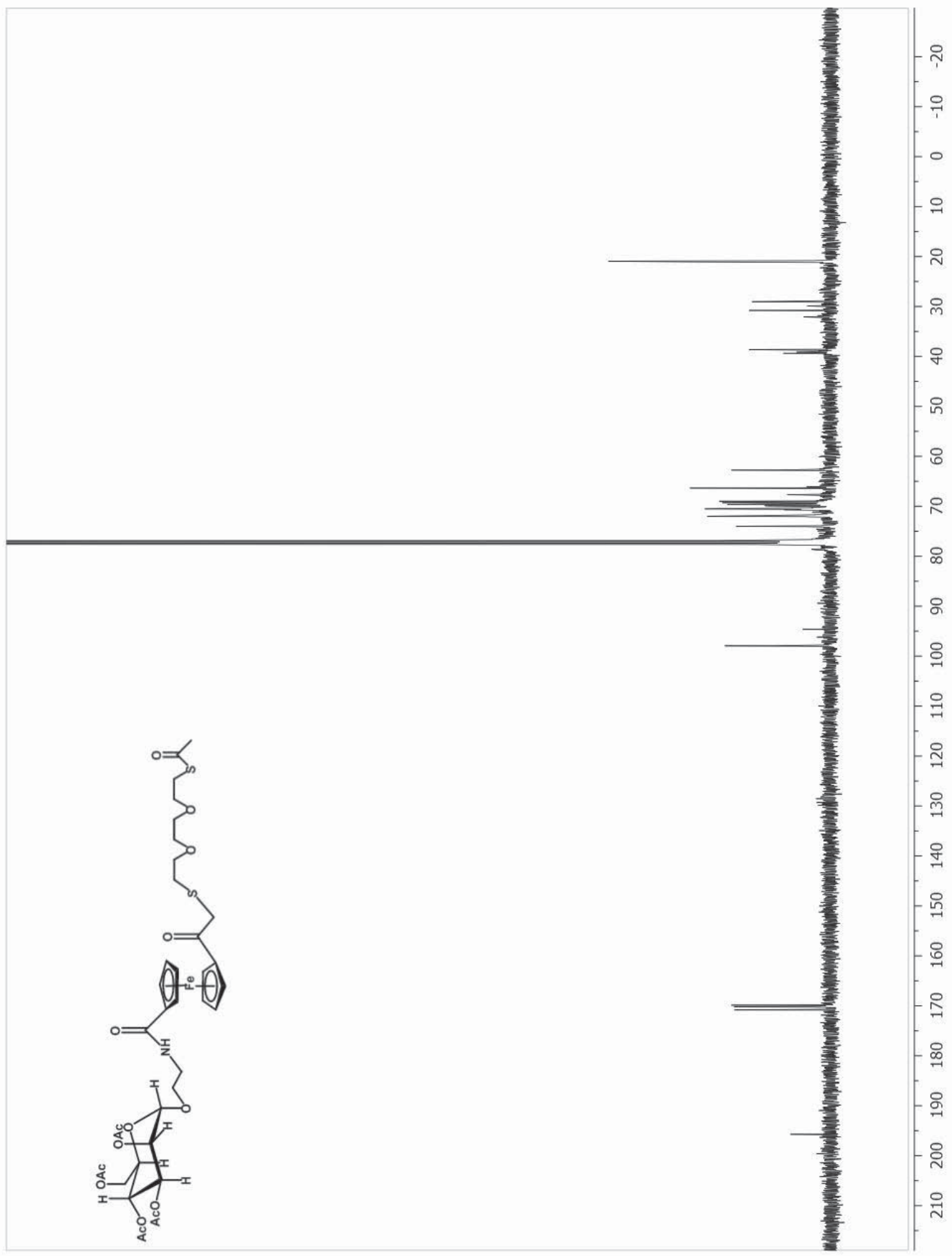

Figure E.14. ${ }^{13} \mathrm{C}$ NMR spectrum of compound 10 


\section{APPENDIX F}

\section{Copyright permissions}

Chapter 2 of this dissertation was published in the ACS Appl. Mater. Interfaces, 2013, Copyright $(\subseteq)$ 2013, American Chemical Society. As the first author, I am allowed to "include the article in full or in part in my thesis or dissertation". Below are the excerpts from the American Chemical Society copyright declaration for authors.

\section{For Chapter 2}

Copyright

Clearance

Center

ACS Publications Title:

High quality High impact.

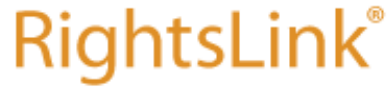

Highly Water-Soluble BODIPY-

Based Fluorescent Probe for

Sensitive and Selective

Detection of Nitric Oxide in Living Cells

Author:

Giri K. Vegesna, Srinivas R. Sripathi, Jingtuo Zhang, Shilei Zhu, weilue He, Fen-Tair Luo, Wan Jin Jahng, Megan Frost, and Haiying Liu

Publication: Applied Materials

Publisher: American Chemical Society

Date: $\quad$ May 1, 2013

Copyright (c) 2013, American Chemical Society

\section{PERMISSION/LICENSE IS GRANTED FOR YOUR ORDER AT NO CHARGE}

This type of permission/license, instead of the standard Terms \& Conditions, is sent to you because no fee is being charged for your order. Please note the following:

- Permission is granted for your request in both print and electronic formats, and translations.

- If figures and/or tables were requested, they may be adapted or used in part.

- Please print this page for your records and send a copy of it to your publisher/graduate school.

- Appropriate credit for the requested material should be given as follows: "Reprinted (adapted) with permission from (COMPLETE REFERENCE CITATION). Copyright (YEAR) American Chemical Society." Insert appropriate information in place of the capitalized words.

- One-time permission is granted only for the use specified in your request. No additional uses are granted (such as derivative works or other editions). For any other uses, please submit a new request. 
Chapter 5 of this dissertation was published in the Biosensors and Bioelectronics, 2013, Copyright (C) 2013 Elsevier. As an author, I am allowed to "include the article in full or in part in my thesis or dissertation". Below are the excerpts from the Elsevier copyright declaration for authors.

\title{
For Chapter 5
}

Copyright Clearance

Center

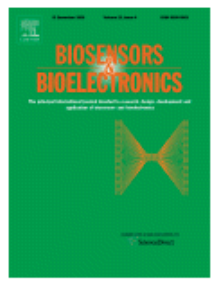

\author{
Title: Glycosylated aniline polymer \\ sensor: Amine to imine \\ conversion on protein- \\ carbohydrate binding \\ Author: $\quad$ Zhe Wang,Chunyan Sun,Giri \\ Vegesna,Haiying Liu,Yang \\ Liu,Jinghong Li,Xiangqun Zeng \\ Publication: Biosensors and Bioelectronics \\ Publisher: Elsevier \\ Date: 15 August 2013 \\ Copyright (s) 2013, Elsevier
}

Order Completed

Thank you very much for your order.

This is a License Agreement between giri K vegesna ("You") and Elsevier ("Elsevier"). The license consists of your order details, the terms and conditions provided by Elsevier, and the payment terms and conditions.

Get the printable license.

\begin{tabular}{|c|c|}
\hline License Number & 3373860059845 \\
\hline License date & Apr 21, 2014 \\
\hline Licensed content publisher & Elsevier \\
\hline $\begin{array}{l}\text { Licensed content } \\
\text { publication }\end{array}$ & Biosensors and Bioelectronics \\
\hline Licensed content title & $\begin{array}{l}\text { Glycosylated aniline polymer sensor: Amine to imine conversion on protein- } \\
\text { carbohydrate binding }\end{array}$ \\
\hline Licensed content author & $\begin{array}{l}\text { Zhe Wang, Chunyan Sun, Giri Vegesna, Haiying Liu,Yang Liu,Jinghong Li,Xiangqun } \\
\text { Zeng }\end{array}$ \\
\hline Licensed content date & 15 August 2013 \\
\hline $\begin{array}{l}\text { Licensed content volume } \\
\text { number }\end{array}$ & 46 \\
\hline Number of pages & 7 \\
\hline Type of Use & reuse in a thesis/dissertation \\
\hline Portion & full article \\
\hline Format & both print and electronic \\
\hline $\begin{array}{l}\text { Are you the author of this } \\
\text { Elsevier article? }\end{array}$ & Yes \\
\hline Will you be translating? & No \\
\hline $\begin{array}{l}\text { Title of your } \\
\text { thesis/dissertation }\end{array}$ & $\begin{array}{l}\text { DESIGN, SYNTHESIS AND APPLICATIONS OF FLUORESCENT AND ELECTROCHEMICAL } \\
\text { PROBES }\end{array}$ \\
\hline Expected completion date & Apr 2014 \\
\hline $\begin{array}{l}\text { Estimated size (number of } \\
\text { pages) }\end{array}$ & 220 \\
\hline Elsevier VAT number & GB 494627212 \\
\hline Permissions price & 0.00 USD \\
\hline VAT/Local Sales Tax & 0.00 USD / $0.00 \mathrm{GBP}$ \\
\hline Total & 0.00 USD \\
\hline
\end{tabular}

Copyright (e) 2014 Copyright Clearance Center, Inc. All Rights Reserved. Privacy statement. Comments? We would like to hear from you. E-mail us at customercare@copyright.com 